$$
\begin{gathered}
\text { Universidade de São Paulo } \\
\text { Instituto de Física de São Carlos }
\end{gathered}
$$

Krissia de Zawadzki

\title{
Relaxação magnética em ligas magnéticas diluídas
}





\section{Relaxação magnética em ligas magnéticas diluídas}

Dissertação apresentada ao Programa de PósGraduação em Física do Instituto de Física de São Carlos da Universidade de São Paulo, para obtenção do título de mestre em Ciências.

Área de Concentração: Física Aplicada Opção:

Física Computacional

Orientador: Prof. Dr. Luiz Nunes de Oliveira

\section{Versão Corrigida}

(versão original disponível na Unidade que aloja o Programa)

São Carlos 
AUTORIZO A REPRODUÇÃO E DIVULGAÇÃO TOTAL OU PARCIAL DESTE TRABALHO, POR QUALQUER MEIO CONVENCIONAL OU ELETRÔNICO PARA FINS DE ESTUDO E PESQUISA, DESDE QUE CITADA A FONTE.

Ficha catalográfica elaborada pelo Serviço de Biblioteca e Informação do IFSC, com os dados fornecidos pelo(a) autor(a)

de Zawadzki, Krissia

Relaxação magnética em ligas magnéticas diluídas /

Krissia de Zawadzki; orientador Luiz Nunes de

Oliveira - versão corrigida -- São Carlos, 2014. $139 \mathrm{p}$.

Dissertação (Mestrado - Programa de Pós-Graduação em Física Aplicada Computacional) -- Instituto de Física de São Carlos, Universidade de São Paulo, 2014.

1. Efeito Kondo. 2. Propriedades magnéticas. 3.

Nuvem Kondo. I. Nunes de Oliveira, Luiz, orient. II. Título. 


\section{AGRADECIMENTOS}

Esta dissertação é dedicada a todas as pessoas que contribuíram para que eu conseguisse chegar à pós-graduação e àqueles que tornaram esses dois anos um momento bastante especial em minha existência. Peço desculpas antecipadamente caso eu esqueça alguém, já que a lista de pessoas para agradecer é deveras grande.

Primeiramente, eu gostaria de agradecer ao Prof. Dr. Luiz Nunes de Oliveira pela orientação e, principalmente, pela paciência. Com sua capacidade de explicar qualquer coisa de forma inteligível (quase uma bruxaria que só ele é capaz de fazer), Luiz é um exemplo de pesquisador, professor e pessoa. Além disso, foi ele quem incentivou meus primeiros passos na pesquisa acadêmica, sem os quais eu não teria entusiasmo e motivação para prosseguir. Sinto-me infinitamente honrada com a oportunidade de trabalhar em seu grupo de pesquisa.

Agradeço especialmente à minha pequena família, que hoje compreende minha mãe, tia, primos e irmã gêmea Andressa. À minha irmã, particularmente, por ouvir-me nos momentos de dificuldade e converter nossa semelhança em motivação para que eu buscasse cada vez mais conhecimento. Se estivessem aqui, eu agradeceria ao meu pai, meu irmão Eduardo e às minhas avós Ana e Regina (in memorium) pelas reminiscências de minha feliz infância. Eu também destaco aqui um amigo cuja constante presença o tornou parte de minha família: Vinicius (ou Pé de Pano) você é uma das pessoas mais inteligentes e admiráveis que conheci. Obrigada pela paciência em lidar diariamente com minhas falas intermináveis e por esclarecer alegremente conceitos obscuros em temas diversos. Apenas conseguirei superar sua falta nos anos vindouros, pensando que você será tão notável como médico quanto é como físico.

Acho oportuno homenagear todos os docentes que contribuíram para minha formação. Em especial, os professores Totó, Gonzalo, Agostinho, Paulo Miranda, Alcaraz, Valter, Lidério, Reginaldo, Leornardo e Miled pelas aulas didáticas e interessantes e a professora Tereza pelos aconselhamentos. Gostaria também de retribuir a atenção do professor Tito quando fui sua monitora de laboratório e à turma de Engenharia da Computação que me recebeu muito bem nesta tarefa.

Ao professor Luciano, agradeço a oportunidade de ter trabalhado como aluna de iniciação científica e pelos ensinamentos culturais. Aproveito para incluir aqui os colegas do antigo Grupo de Visão Cibernética: Débora, Mauro, Matheus, Thomas, Lucas, Renato, Cesar e Mônica todos vocês são especiais para mim. 
Agradeço ao meu colega Paulo Matias por toda sua gentileza e disponibilidade ao longo de minha jornada no curso de Física Computacional. Eu diria que Paulo foi minha grande inspiração para iniciar e concluir a graduação, além de ter feito com que eu superasse diversas dificuldades computacionais. Seu conhecimento e suas fantásticas dicas fizeram da computação uma ferramenta indispensável em meu trabalho.

Tributo sinceros reconhecimentos à companhia dos colegas Raul e Celso nos cafés da tarde e nos cursos de pós-graduação. Aprecio deveras nossas conversas nessa última etapa de finalização das dissertações, pois elas foram fundamentais para criar uma expectativa mais positiva e atenuar a ansiedade do momento. Torço pelo sucesso de suas pesquisas no doutorado e espero que possamos manter o hábito da cafeína vespertina.

Agradeço aos meus amigos Heitor e Joseana (a nossa McGyver) por partilhar momentos doces (ou, como chamamos, de gordice) nos intervalos dos estudos. Aos colegas Hudson e Thiago, pelas conversas informais nas quais discutimos física e assuntos aleatórios. Ao colega Thiago Mosqueiro agradeço imensamente por disponibilizar o pacote LaTeX utilizado nesta dissertação. Também quero retribuir a felicidade de conviver com pessoas engajadas e verdadeiramente amigas como Luísa, Carla, Thereza e Marta.

Aos membros do grupo "Galera do Cinema", gostaria de agradecer pelos finais de semana de entretenimento e distração e à Comissão da quarta Semana Integrada SIFSC por aceitar-me neste ano como colaboradora. Ao meus colegas Tiago, Paulo, Bruno, Diego, Felipe (clap, clap, clap), Ciro, Prati e Karen, com os quais tive a honra de "atuar"no Grupo de Teatro Atuando em Psi $(\mathrm{GT}|\psi\rangle)$, agradeço por recordarem de minha pessoa em toda apresentação, mesmo eu tendo os deixado para dedicar-me mais à pesquisa.

Quero homenagear alguns funcionários do IFSC, especialmente a Beth, que me ajudou muito na época de graduação, a Cris, que eu destacaria pela eficiência para resolver as burocracias do grupo, e as bibliotecárias Cristina e Neusa, que corrigiram a dissertação adequando-a às normas técnicas.

Devo agradecer à FAPESP pelo apoio financeiro deste projeto.

Finalmente, gostaria de agradecer à banca examinadora pela disponibilidade para avaliar este trabalho. 
The fundamental laws necessary for the mathematical treatment of a large part of physics and the whole of chemistry are thus completely known, and the difficulty lies only in the fact that application of these laws leads to equations that are too complex to be solved.

Paul Dirac. 

Esta dissertação foi financiada pela FAPESP. 



\section{RESUMO}

ZAWADZKI, K. Relaxação magnética em ligas magnéticas diluídas. 2014. 139 p. Dissertação (Mestrado em Ciências) - Instituto de Física de São Carlos, Universidade de São Paulo, São Carlos, 2014.

Na década de 60, Kondo mostrou que o mínimo de resistividade observado em alguns metais a baixas temperaturas é devido ao acoplamento antiferromagnetico entre impurezas e a banda de condução do metal hospedeiro. Embora muitos resultados teóricos e experimentais tenham sido obtidos desde então, uma interessante questão remanesce: a estrutura da nuvem de elétrons de condução que blinda o momento magnético da impureza. Com o objetivo de estudar essa estrutura, apresentamos um procedimento de Grupo de Renormalização Numérico (NRG) para computar a taxa NMR de relaxação longitudinal $1 / T_{1}$ de uma ponta de prova como função da temperatura e da distância $R$ entre a ponta e a impureza. Introduzimos uma base quântica contendo dois conjuntos de estados de condução. Os elementos de um dos conjuntos, denotados $f_{n}$, são ondas $s$ acopladas à impureza e descritas pelo Hamiltoniano de Anderson, que pode ser diagonalizado pelo procedimento tradicional de NRG. Cada elemento do segundo subconjunto, denotado $\bar{c}_{\varepsilon}$, é uma combinação linear de um estado de onda $s$ centrado na ponta de prova com os $f_{n}$ 's, de modo que os $\bar{c}_{\varepsilon}$ são ortogonais aos $f_{n}$ 's. Diferente dos $f_{n}$ 's, os $\bar{c}_{\varepsilon}$ são desacoplados da impureza. Com base nessas definições, mostramos que $1 / T_{1}$ tem três componentes, que chamamos escalar, vetorial e matricial. A componente escalar, associada com os estados $\bar{c}_{\varepsilon}$ espalhados pela ponta de prova, é independente da temperatura e fracamente dependente de $R$. A componente $\left(1 / T_{1}\right)_{\text {mat }}$, associada com os elétrons $f_{n}$, decai rapidamente com $R$. Damos atenção especial à componente vetorial $\left(1 / T_{1}\right)_{\text {vet }}$, que está associada ao espalhamento cruzado de canais $f_{n}$ e $\bar{c}_{\varepsilon}$, e domina para distâncias $R$ grandes. A dependência térmica da taxa de relaxação mostra que há uma mudança no comportamento da curva quando passamos por $R_{K} \propto T_{K}^{-1}$. No limite de altas temperaturas, observamos que $\left(1 / T_{1}\right)_{\text {vet }}(T)$ pode ser mapeada nas curvas de condutância $\mathcal{G}_{\text {side }}\left(T / T_{K}\right)$ e $\mathcal{G}_{S E T}\left(T / T_{K}\right)$. Com respeito à dependência espacial, analisamos as oscilações de Friedel. A partir desses resultados verificamos a relação $R_{K}=\hbar v_{F} / K_{B} T_{K}$ e mostramos que as fases das oscilações de Fridel mudam quando olhamos para o interior e o exterior da nuvem. 
Palavras-chave: Efeito Kondo. Propriedades magnéticas. Nuvem Kondo. 


\section{ABSTRACT}

ZAWADZKI, K. Magnetic relaxation in dilute magnetic alloys. 2014. 139 p. Dissertação (Mestrado em Ciências) - Instituto de Física de São Carlos, Universidade de São Paulo, São Carlos, 2014.

In the 1960's, Kondo showed that the resistivity minimum observed in a number of metals at low temperatures is due to the antiferromagnetic coupling between magnetic impurities and the conduction electrons of the metallic host. Although many theoretical and experimental results have been obtained since then, an interesting question remains unanswered: the structure of the cloud of conduction electrons that screen the magnetic moment of the impurity. To add insight into that structure, we here present a Numerical Renormalization Group (NRG) procedure to compute the NMR longitudinal relaxation rate $1 / T_{1}$ of a probe at distance $R$ from the impurity, as a function of $R$ and of the temperature. We define a quantum basis containing two subsets of conduction states. The elements of one the subsets, denoted $f_{n}$, are $s$-wave states coupled to the impurity and described by the Anderson Hamiltonian, which can be diagonalized by the traditional NRG procedure. Each element of the second subset, denoted $\bar{c}_{\epsilon}$ is a linear combination of an $s$-wave state centered at the probe with the $f_{n}$ 's, the combination constructed to make $\bar{c}_{\epsilon}$ orthogonal to the $f_{n}$ 's. By contrast with the $f_{n}$ 's, the $\bar{c}_{\epsilon}{ }^{\prime} \mathrm{s}$ are decoupled from the impurity. On the basis of these definitions, we show that $1 / T_{1}$ has three components, which we name scalar, vector, and matrix. The scalar component, associated with the scattering of $\bar{c}_{\epsilon}$ states off the probe, is temperature independent and weakly dependent on $R$. The matrix component $\left(1 / T_{1}\right)_{\text {mat }}$, associated with the scattering of $f_{n}$ electrons, decays rapidly with $R$. The vector component $\left(1 / T_{1}\right)_{\text {vet }}$ is due to cross-chanel scattering between the $f_{n}$ and the $\bar{c}_{\epsilon}$ subsets. We give central attention to the latter, which is dominant over $\left(1 / T_{1}\right)_{\text {mat }}$ at large distances $R$. The $\mathrm{T}$-dependence of the relaxation rate changes as we cross $R_{K} \propto T_{K}^{-1}$. At high temperatures limit, we observe the $\left(1 / T_{1}\right)_{\text {vet }}(T)$ mapping in the universal conductance curves $\mathcal{G}_{\text {side }}\left(T / T_{K}\right)$ e $\mathcal{G}_{S E T}\left(T / T_{K}\right)$. Regarding the spatial dependence, we analize the Friedel oscillations. From our results we verify the relation $R_{K}=\hbar v_{F} / K_{B} T_{K}$ for the radius of the Kondo screening cloud and also show that the phase of the Friedel oscillations changes as we are inside or outside the cloud. launch the idea of a shell around $R_{K}$ where screening effects remain important. 
Keywords: Kondo effect. Magnetic properties. Kondo screening cloud. 


\section{LISTA DE FIGURAS}

Figura 1.1 - Comportamento resistivo de materiais metálicos. a) A medida que a temperatura no metal é reduzida, sua resistência diminui até alcançar um valor residual (azul). Certos materiais metálicos se tornam supercondutores a partir de uma temperatura crítica (verde). Em ligas metálicas, tal como $\mathrm{CoCu}$, a presença de impurezas dá origem ao Efeito Kondo, fazendo com que a resistência aumente com a redução da temperatura (vermelho). b) Pontos quânticos podem ser criados artificialmente em semicondutores dispondo um spin localizado entre fios metálicos. Sistemas dessa natureza permitem controlar o número de elétrons. Se esse número for ímpar, a condutância medida entre os dois fios aumenta a baixas temperaturas devido ao efeito Kondo (vermelho). Este comportamento não é verificado para um número par de elétrons, já que a condutância decresce a baixas temperaturas (azul). Fonte: KOUWENHOVEN, L. P. (1). . . . . . . . . . . . . . . . 26

Figura 1.2 - Resistividade $\rho$ em função da temperatura $T$. Comparação da curva experimental $\rho-T$ de ligas diluídas de $A u F e$ com os resultados do modelo de Kondo em seu trabalho de 1964. Fonte: KONDO, J. (2).

Figura 1.3 - Ilustração de pontos quânticos. a) Esses dispositivos de escala nanomêtrica consistem de eletrodos usualmente construídos por técnicas avançadas de epitaxia em nanocristais, por deposição iônica ou por litografia. Aplicando voltagens aos eletrodos metálicos é possível confinar elétrons em uma pequena região, formando pontos quânticos. Fonte: GOLDHABER-GORDON, D. (3). b) Um segundo exemplo, desta vez constituído por dois pontos quânticos. Pontos quânticos duplos são formados conectando dois pontos quânticos. Um efeito interessante decorre de pontos quânticos duplos: um dos pontos está no estado de Kondo, de modo que o spin do elétron confinado é compensado pelo spin dos elétrons ao redor. Fonte: ScienceDaily (4). . . . . . . . . . . 28

Figura 1.4 - Nuvem Kondo em um sistema metálico infinito e isotrópico com a impureza localizada na origem. Fonte: elaborada pela autora. 
Figura 2.1 - Metal hospedeiro semi-infinito na direção $z$ com a impureza localizada à distância $\vec{d}$ do plano limitante $z=0$. Fonte: elaborada pela autora. . 34

Figura 2.2 - Para $R_{K}<d$ a nuvem Kondo não há truncamento pela superfície, de modo que o comportamento esperado é o de uma nuvem com raio inversamente proporcional a $T_{K}$, como descrito em (5). Fonte: elaborada pela autora. . . . . . . . . . . . . . . . . . . . . . 34

Figura 2.3 - Para $R_{K}>d$ a nuvem Kondo é truncada pela superfície e seu tamanho dependeria da geometria do sistema. Fonte: elaborada pela autora. . . 35

Figura 2.4 - A relação de dispersão para as energias da banda de condução no intervalo $[-D, D]$ é aproximada por uma função linear, simétrica em torno do momento de Fermi $k_{F}$. Fonte: elaborada pela autora. . . . . . . . . 36

Figura 2.5 - Representação dos níveis de energia no Modelo de Anderson simétrico. Nesta situação $\varepsilon_{d}=-U / 2$, e o estado da impureza pode assumir quatro configurações: vazia $\left(n_{d}=0\right)$, semi ocupada com um elétron com spin up ou down $\left(n_{d}=1\right)$ e cheia com dois elétrons com spins opostos $\left(n_{d}=2\right)$. Fonte: elaborada pela autora.

Figura 2.6 - Transições virtuais da impureza no regime de acoplamento forte para o Modelo de Anderson simétrico. Nívels da banda de condução são representados em dourado, enquanto a impureza é ilustrada em prateado. a) Estado inicial da impureza no nível $\varepsilon_{d}$ com spin up. b) Uma vez que adicionar um elétron não é energeticamente possível por conta do valor de $U$, o elétron up da impureza ocupa um estado virtual e então é substituído por um elétron do metal. c) Da transição virtual resulta o flip (inversão) de spin da impureza. Fonte: elaborada pela autora. . . 38

Figura 3.1 - Cascas concêntricas em torno da impureza representando os estados na base Kondo. Fonte: WILSON, K. G. (10). . . . . . . . . . . . . . . . 49

Figura 3.2 - A banda de condução é discretizada em escala logarítimica, definida por um parâmetro adimensional $\Lambda$. Fonte: elaborada pela autora.

Figura 3.3 - Representação do hopping de elétrons entre os sítios $f_{n}$ da banda de condução. Cada elétron em um sítio $f_{n}$ pode ser transferido para um sítio $f_{n+1}$ e vice-versa. Fonte: elaborada pela autora. . . . . . . . . . . 55 
Figura 3.4 - Representação dos 4 estados de muitos corpos na iteração $N=-1$. Há apenas o sítio da impureza com quatro possíveis configurações eletrônicas: cada estado $|0\rangle, c_{d \uparrow}^{\dagger}|0\rangle, c_{d_{\downarrow}}^{\dagger}|0\rangle$ and $c_{d \uparrow}^{\dagger} c_{d \downarrow}^{\dagger}|0\rangle$ pode ter, respectivamente, 0 elétrons, 1 elétron com spin up, um elétron com spin down, ou dois elétrons com spins opostos. Fonte: elaborada pela autora. 57

Figura 3.5 - Ilustração dos 16 estados de muitos corpos na iteração $N=0$. Nesta iteração, participam os sítios $c_{d}$ e $f_{0}$, e o estado de maior carga possui dois elétrons em cada um destes sítio. Os estados que não foram identificados como $\left|Q, S, S_{z}, r\right\rangle$ correspondem às combinações singleto e tripleto de $c_{d \uparrow}^{\dagger} f_{0 \downarrow}^{\dagger}|0\rangle$ e $c_{d_{\downarrow}}^{\dagger} f_{0 \uparrow}^{\dagger}|0\rangle$. Fonte: elaborada pela autora. . . . . . 58

Figura 3.6 - Ciclo iterativo do NRG na solução do problema Kondo. A partir da solução analítica na iteração $N=-1$, construímos a base primitiva da iteração $N=0$ que inicia o ciclo iterativo. No restante das iterações $N$, construímos a base primitiva na qual o Hamiltoniano $H_{N}$ é diagonalizado. Os autovalores e autovetores de $H_{N}$ são armazenados para o cálculo de propriedades físicas e para a construção da iteração $N+1$. Fonte: elaborada pela autora.

Figura 3.7 - Setores ativos (em azul) para a iteração $N=6$. A carga máxima é $Q_{\max }^{5}=7$. Para $Q=0$, o conjunto de spins permitidos é $[0,1,2,3]$, enquanto para $Q=7$ só é permitido $S=1$. Em uma iteração par setores de carga par têm spins pares e setores de carga ímpar têm spins ímpares. Fonte: elaborada pela autora. 
Figura 3.8 - Construção da base primitiva de um setor $(Q, S)$ na iteração $N$ a partir dos estados da iteração $N-1$. Setores pais e filhos são ilustrados em vermelho e verde, respectivamente. a) Seja $\left\{\left|Q, S, S_{z}, l\right\rangle\right\}$ a base de estados do setor $(Q, S)$ da iteração $N-1$. A atuação dos os operadores $O_{g}(g=\mathcal{S}, \mathcal{W}, \mathcal{E}, \mathcal{N})$ nessa base dá origem aos estados filhos nos respectivos setores $(q-1, s),(q, s+1 / 2),(q+1, s)$ e $(q, s-1 / 2)$ na iteração $N$. Os gêneros atribuídos aos operadores $O_{g}$ correspondem à localização geográfica dos setores filhos de $Q, S$ : norte $(\mathcal{N})$, leste $(\mathcal{E})$, sul $(\mathcal{S})$ e oeste $(\mathcal{W})$. b) A base primitiva de um setor filho $(q, s)$ na iteração $N$ é implementada através da herança de estados dos setores pais oriundos da iteração $N-1$. O setor $(q, s)$ recebe estados dos setores $(Q-1, S)$ via $O_{\mathcal{S}},(Q, S+1 / 2)$ via $O_{\mathcal{W}},(Q+1, S)$ via $O \mathcal{N}$ e $(Q, S-1 / 2)$ via $O_{\mathcal{W}}$

Figura 3.9 - Setores pais (vermelho) e filhos (azul) nas iterações $N=-1$ e $N=$ 0 . A iteração $N=-1$ é composta pelos setores $(Q=1, S=0)$, $\left(Q=0, S=\frac{1}{2}\right)$ e $(Q=-1, S=0)$, cada um contendo um estado. Esses estados são herdados na iteração $N=0$, dando origem à base primitiva dos setores $(Q=2, S=0),\left(Q=1, S=\frac{1}{2}\right),(Q=0, S=0)$, $\left(Q=-1, S=\frac{1}{2}\right)$ e $(Q=-2, S=0)$. Na iteração $N=1$, os setores filhos herdam os estados em $N=0$ cujos autovalores são inferiores a $E_{U V}$. Fonte: elaborada pela autora.

Figura 3.10 -Esquema de cálculo dos invariantes $N E$ e $N W$ de um setor $(Q, S)$ com os setores $\left(Q+1, S+\frac{1}{2}\right)$ e $\left(Q+1, S-\frac{1}{2}\right)$, respectivamente. Os setores circulados em vermelho correspondem aos pais de $(Q, S)$ existentes na iteração anterior. a) Os invariantes nordeste $\left\langle q+1, s+1 / 2, r^{\prime}|| f_{N} \| q, s, r\right\rangle$ recebem a contribuição de estados com origem nos setores pais $(Q+$ $1, S)$ e $\left(Q, S+\frac{1}{2}\right)$. b) Os invariantes noroeste $\left\langle q+1, s-1 / 2, r^{\prime}\left\|f_{N}\right\| q, s, r\right\rangle$ recebem a contribuição de estados com origem nos setores pais $(Q+$ $1, S)$ e $\left(Q, S-\frac{1}{2}\right)$. Fonte: elaborada pela autora. . . . . . . . . . . 69 
Figura 3.11 -Cálculo dos invariantes entre os estados da iteração $N=-1$. a)Os estados $|Q=0, S=1 / 2,1\rangle$ e $|Q=1, S=0,1\rangle$ juntos compõe o elemento de matriz $\left\langle Q=1, S=0, r^{\prime}=1\left\|f_{-1}\right\| Q=0, S=1 / 2, r=1\right\rangle$ na direção $N W$ do setor $(Q=0, S=1 / 2)$. b) Por sua vez, o invariante $N E$ do setor $(Q=-1, S=0)$ é construído a partir dos estados $|Q=-1, S=0, r=1\rangle$ e $\left|Q=0, S=1 / 2, r^{\prime}=1\right\rangle$. Fonte: elaborada pela autora.

Figura 4.1 - Disposição dos elementos invariantes $\left\langle\varphi|| f_{n}^{\dagger} f_{m} \| \psi\right\rangle$ em uma matriz de $n$ linhas e $m$ colunas, com $m, n=0, \ldots, N$. Cada elemento $\left\langle\varphi\left\|f_{n}^{\dagger} f_{m}\right\| \psi\right\rangle$ pode ser determinado pelos vizinhos $\left\langle\varphi\left\|f_{n \pm 1}^{\dagger} f_{m \pm 1}\right\| \psi\right\rangle$. Fonte: elaborada pela autora.

Figura 4.2 - Cálculo recursivo dos elementos de matriz reduzidos $\left\langle\varphi\left\|f_{n}^{\dagger} f_{m}\right\| \psi\right\rangle$ quando $|\varphi\rangle=|\psi\rangle$. No início da computação recursiva as linhas e colunas coloridas são conhecidas: a linha colorida em vermelho corresponde aos elementos de matriz da forma $\left\langle\varphi\left\|f_{N}^{\dagger} f_{m}\right\| \psi\right\rangle(m=0, \ldots, N)$ e a coluna mostrada em azul diz respeito aos invariantes $\left\langle\varphi\left\|f_{n}^{\dagger} f_{0}\right\| \psi\right\rangle$ $(n=0, \ldots, N)$. Para obter um invariante iii da linha $N-1$ (circulado em vermelho) precisamos conhecer seus vizinhos $i$ ao noroeste e $i i$ ao nordeste, ambos localizados na linha $N$, aplicando a equação (4.33). Analogamente, para determinar um elemento iv na linha $N-2$ (circulado em azul) devemos ter além de seus vizinhos noroeste $i i$ e nordeste iii da linha $N-1$ o invariante $i$ da linha $N$. Com a equação (4.34) é possível determinar todos os elementos da mesma linha. Fonte: elaborada pela autora.

Figura 5.1 - Coeficientes $u_{10, m}$ e $u_{9, m}$ para $\Lambda=3.0$. O valor absoluto é máximo quando $m=n / 2$. Para $m>n / 2$, os coeficientes decaem à taxa $\Lambda^{(n / 2-m) / 2}$. O valor máximo de $n$ é definido pelos parâmetros do modelo, $E_{\min }$ e $\Lambda$, enquanto $m$ é arbitrariamente grande. O mesmo gráfico para $n>4$ revela que os $u_{n m}$ dependem somente da diferença $n / 2-m$.

Fonte: elaborada pela autora.

Figura 5.2 - Coeficientes $\gamma_{n}(R)$ para $k_{F} R=n \pi$ com $n \in[1000,100,10,1]$. O número $n$ de iteração característico $\mathcal{N}_{k_{F} R}$ no qual $\gamma_{n}$ é máximo aparece destacado para cada um dos casos mostrados na figura. Fonte: elaborada pela autora. 
Figura 5.3 - Coeficientes $\gamma_{n}(R)$ para $k_{F} R=\left(n+\frac{1}{2}\right) \pi$ com $n \in[1000,100,10,1]$. Para cada valor de $k_{F} R$ destacamos a iteração característica $\mathcal{N}_{k_{F} R}$, calculada através da equação (5.15). Conforme $k_{F} R$ fica menor, a contribuição dos coeficientes $\gamma_{0}$ vai se tornando cada vez maior, superando o pico típico em $n=\mathcal{N}_{k_{F} R}$. Comparando estes resultados com a figura 5.2, notamos que esse efeito é mais proeminente para valores $k_{F} R$ múltiplos de $\frac{\pi}{2}$, conforme esperado pela expansão dos $\gamma_{n}$ na equação (5.10). Fonte: elaborada pela autora. . . . . . . . . . . . . . . . . 96

Figura 5.4 - Oscilações de Friedel no ponto fixo de temperatura $T \ll T_{K}$. Pontos destacados com setas correspondem aos máximos e aos nós das oscilações. O ponto $(n+1 / 4) \pi$ combina os comportamentos destrutivo $\mathrm{e}$ construtivo. Fonte: elaborada pela autora.

Figura 5.5 - Contribuição vetorial para a taxa de relaxação $1 / T_{1}$ com $k_{F} R=(n+$ $\left.\frac{1}{2}\right) \pi$. A correspondente parcela da taxa é proporcional a $\left(k_{F} R\right)^{2}$ e decai para baixas temperaturas. A pequenas distâncias, no limite de altas temperaturas, a curva pode ser mapeada na condutância $\mathcal{G}_{\text {side }}$ de um dispositivo formato $\mathrm{T}$. Fonte: elaborada pela autora.

Figura 5.6 - Contribuição para a taxa de relaxação $1 / T_{1}$. com $k_{F} R=n \pi$. A contribuição começa muito pequena para altas temperaturas e aumenta até atingir um patamar constante em $T<T_{K}$. O comportamento global é análogo ao da condutância $\mathcal{G}_{S E T}$ de um transístor de um elétron. Fonte: elaborada pela autora. . . . . . . . . . . . . . . . . 100

Figura 5.7 - Taxa de relaxação $\left(1 / T_{1}\right)_{\text {vet }}$ em função da temperatura na ausência de oscilação. A altas temperatuas, a componente vetorial de $1 / T_{1}$ mescla os comportamentos das condutâncias $\mathcal{G}_{\text {side }}$ e $\mathcal{G}_{S E T}$. A mudança de comportamento ocorre nas vizinhanças do raio da nuvem Kondo $R_{K}=$ $\hbar v_{F} / k_{B} T_{K}$, confirmando a hipótese de que o raio seria encontrado em uma escala de distância mesoscópica. Fonte: elaborada pela autora. . 101

Figura 5.8 - Taxa de relaxação em função da temperatura e os limites da nuvem Kondo. Para $R \gg R_{K}$ a dependência térmica é análoga a da curva $\mathcal{G}_{S E T}$, enquanto para $R \ll R_{K}$ ela pode ser mapeada na condutância $\mathcal{G}_{\text {side }}$. Fonte: elaborada pela autora. . . . . . . . . . . . . . . . . . . 102 
Figura A.1 - Implementação da completeza (A.1) para o cálculo dos elementos de matriz $\left\langle\varphi\left|f_{n \mu}^{\dagger} f_{m \nu}\right| \psi\right\rangle$. Os estados $|\varphi\rangle$ e $|\psi\rangle$ pertencem ao mesmo setor $(q, s)$, enquanto os estados $|\xi\rangle$ podem estar a sudeste $S E$ ou sudoeste $S W$. Fonte: elaborada pela autora.

Figura B.1 - Setores contendo estados que contribuem direta ou indiretamente para o invariante $\left\langle q, s+1, r^{\prime}\left\|f_{N}^{\dagger} f_{N}\right\| q, s, r\right\rangle$. Uma vez que os dois estados primitivos (coloridos em laranja) devem ter o mesmo pai, o estado que Ihes deu origem deve pertencer ao setor $(q, s+1 / 2)$ (indicado pelo rótulo inclinado $s+1 / 2$ ). As abas representam os gêneros dos estados primitivos. Fonte: elaborada pela autora. . . . . . . . . . . . . . . . 125

Figura B.2 - Setores envolvidos no cálculo dos elementos de matriz $\left\langle q, s-1, r^{\prime}|| f_{N}^{\dagger} f_{N} \| q, s, r\right\rangle$. Quando o invariante deve ser tomado entre um ket e um bra de gêneros $\mathcal{E}$ e $\mathcal{W}$, respectivamente, estes foram herdados de estado da iteração $N-1$ que pertencia ao setor $(q, s+1 / 2)$. Fonte: elaborada pela autora. 126

Figura B.3 - Setores envolvidos no cálculo dos elementos de matriz da equação (B.34) para estados com gêneros $g_{p^{\prime}}=g_{p}=\mathcal{E}$. O invariante $\left\langle q, s, p^{\prime}\left\|f_{0}^{\dagger} f_{n}\right\| q, s, p\right\rangle$ é tomado entre estados do mesmo setor $(q, s)$ herdados de $(q, s-1 / 2)$ no início do ciclo iterativo. Fonte: elaborada pela autora. . . . . . . . . 129

Figura B.4 - Casos especiais de herança para os quais o invariante da da equação (B.34) é não-nulo. São mostrados os seguintes pares de gêneros $g_{p^{\prime}} g_{p}$ : a) $\mathcal{W}$-W , b) $\mathcal{W}$-E e b) $\mathcal{E}$-W . Fonte: elaborada pela autora. . . . . . . 131

Figura B.5 - Setores $(q, s)$ e $(q, s+1)$ envolvidos no cálculo da equação (B.41). Mostramos os possíveis gêneros dos estados herdados pelos setores pais (com rótulos inclinados): a) $\mathcal{E}-\mathcal{E}$, b) $\mathcal{W}-\mathcal{W}$ e c) $\mathcal{E}-\mathcal{W}$. Fonte: elaborada pela autora.

Figura B.6 - Setores $(q, s)$ e $(q, s-1)$ cujos estados participam da relação (B.44). Destacamos os casos não-triviais de herança para os gêneros $g_{p^{\prime}}-g_{p}:$ a) $\mathcal{E}-\mathcal{E}$, b) $\mathcal{W}-\mathcal{W}$ e c) $\mathcal{W}-\mathcal{E}$. Fonte: elaborada pela autora. 
Figura C.1 - Geometrias de ponto quântico acoplado com um fio quântico. a) Dispositivo em formato $T$. O círculo laranja refere-se a um ponto quântico acoplado lateralmente a um fio quântico. Os potenciais de gate $V_{d}$ e $V_{w}$ controlam, respectivamente, a ocupação eletrônica do ponto e do orbital de Wannier. Esse dispositivo permite que haja interferência entre a corrente fluindo ao longo do fio (setas brancas) e os elétrons que conseguem passar do fio ao ponto. b) Transistor de um único elétron SET. Um ponto quântico faz a ponte entre dois fios quânticos não-interagentes. Um potencial $V_{d}$ controla a energia no ponto quântico, enquanto os potenciais $V_{w}$ deslocam a energia dos orbitais do fio próximo ao ponto. Fonte: elaborado pela autora. . . . . . . . . . . . 136

Figura C.2 - Condutâncias $\mathcal{G}_{S E T}$ e $\mathcal{G}_{\text {side }}$ como função de $T / T_{K}$ normalizadas pela condutância quântica $\mathcal{G}_{2}$. A curva vermelha mostra a condutância $\mathcal{G}_{\text {side }}$ através do dispositivo lateralmente acoplado. A medida que a temperatura é resfriada, a condutância decai a zero. Por outro lado, a condutância $\mathcal{G}_{S E T}$, mostrada em verde, cresce para $T<T_{K}$ e tende à unidade no limite $T \rightarrow 0$. Note que para $T=T_{K}$ temos $G\left(T / T_{K}\right)=0.5 \mathcal{G}_{2} \mathrm{e}$ as duas condutâncias coincidem. Fonte: elaborado pela autora. . . . . 139 


\section{LISTA DE TABELAS}

Tabela 3.1 - Elementos não-diagonais da matriz Hamiltoniano $H_{N}$ entre os estados $\left|q, s, s_{z}, p^{\prime}\right\rangle_{N}^{g^{\prime}}$ e $\left|q, s, s_{z}, p\right\rangle_{N}^{g}$. Os fatores que aparecem na frente dos invariantes são os coeficientes de Clebsch-Gordan. As combinações não listadas de gêneros $g$ e $g^{\prime}$ são nulas. . . . . . . . . . . . . . . . 68

Tabela 3.2 - Elementos de matriz ${ }^{g^{\prime}}\left\langle q+1, s^{\prime}, s_{z}+\mu, p^{\prime}\left|f_{N \mu}^{\dagger}\right| q, s, s_{z}, p\right\rangle_{N}^{g}$ não nulos a partir dos quais os invariantes são computados. A última coluna apresenta os coeficientes de Clebsch-Gordan para as combinações de gêneros que produzem elementos diferentes de zero. . . . . . . . . . . 70

Tabela 5.1 - Parâmetros de entrada do código adotados no cálculo da taxa de relaxação $\left(1 / T_{1}\right)_{v e t} \ldots \ldots \ldots \ldots$. . . . . . . . . . . . . . . . 98

Tabela A.1 - Coeficientes de Clebsch-Gordan referentes aos elementos de matriz $S W .118$

Tabela A.2 - Coeficientes de Clebsch-Gordan referentes aos elementos de matriz SE. 118

Tabela B.1 - Elementos da matriz de coeficientes $\mathcal{G}$ para as possíveis combinações de gêneros. Embora a matriz seja diagonal, de modo que elementos entre estados com mesmo gênero são pressupostamente diferentes de zero, os elementos que efetivamente são não-nulos têm gêneros $\mathcal{E}$ e $\mathcal{W} .124$

Tabela B.2 - Coeficientes $\alpha_{g p}$ relacionando os elementos de matriz na (B.30). Das possíveis combinações dos gêneros $g=\mathcal{N}, \mathcal{E}, \mathcal{S}, \mathcal{W}$, os elementos nãonulos têm $p^{\prime} \neq p \ldots \ldots \ldots$. . . . . . . . . . . . . . 127

Tabela B.3 - Matriz $\mathcal{A}_{g_{p^{\prime}}, g_{p}}$ na soma à direita da equação (B.34). Além dos termos diagonais, destacamos as combinações $\left(g_{p^{\prime}}, g_{p}\right)=(\mathcal{E}, \mathcal{W})$ e $(\mathcal{W}, \mathcal{E}) . \quad .129$

Tabela B.4 - Matriz de coeficientes $\mathcal{B}_{g_{p^{\prime}}, g_{p}}$ na soma à direita da equação (B.41). . . 132

Tabela B.5 - Coeficientes $\mathcal{C}_{g^{\prime} g}$ da matriz $\mathcal{C}$ definida à direita da equação (B.44). . . . 133 



\section{SUMÁRIO}

1 Introdução

2 O sistema quântico 33

2.1 O Modelo de Anderson de uma impureza . . . . . . . . . . . . . . . . . . . 35

2.2 A ponta de prova . . . . . . . . . . . . . . . . . . . . . 39

2.3 Base contínua . . . . . . . . . . . . . . . . . . . . . . . . . . . . . . . . 40

3 O Grupo de Renormalização numérico $\quad 45$

3.1 Da Teoria de Renormalização ao Grupo de Renormalização Numérico .... 46

3.2 O Grupo de Renormalização . . . . . . . . . . . . . . . . . . . . . . . . . . . 47

3.3 O Grupo de Renormalização Numérico no Problema Kondo . . . . . . . . . . 48

3.4 Discretização da banda de condução . . . . . . . . . . . . . . . . . . . 50

3.5 Mudança de base e Transformação de Lanczos . . . . . . . . . . . . . . . . . 52

3.6 Diagonalização iterativa . . . . . . . . . . . . . . . . . . . 56

3.6.1 Iteração $N=-1 \quad \ldots \ldots \ldots \ldots$

3.6.2 Setores $Q, S$ e base primitiva $\ldots \ldots \ldots \ldots$

3.6.3 Cálculo dos invariantes . . . . . . . . . . . . . . . . . . . . . . . . 69

3.6.4 Cálculo recursivo dos elementos de matriz . . . . . . . . . . . . . . 73

4 A taxa de relaxação magnética longitudinal $\quad 77$

4.1 Calculando a relaxação de um sistema Kondo . . . . . . . . . . . . . . . . . 78

4.2 Hamiltoniano da ponta de prova na base de Lanczos . . . . . . . . . . . . . 79

4.2 .1 Contribuição vetorial . . . . . . . . . . . . . . . . . . . . . 81

4.2 .2 Contribuição matricial . . . . . . . . . . . . . . . . . . . . . 82 
5.1 Coeficientes da transformação de Grupo de Renormalização . . . . . . . . . . . 91

5.2 A taxa de relaxação associada à contribuição vetorial . . . . . . . . . . . . . . . 97

$\begin{array}{lll}6 \text { Conclusão } & 105\end{array}$

$\begin{array}{lr}\text { REFERÊNCIAS } & 109\end{array}$

$\begin{array}{ll}\text { Apêndice A - Relação de completeza } & 115\end{array}$

A.1 Calculando os elementos de matriz de $f_{n \mu}^{\dagger} f_{m \nu}$ por completeza . . . . . . . . 115

Apêndice B - Calculando a contribuição matricial por rotação 121

B.1 Cálculo dos elementos de matriz de $f_{N \uparrow}^{\dagger} f_{N \downarrow} \ldots \ldots \ldots . \ldots . \ldots . \ldots 121$

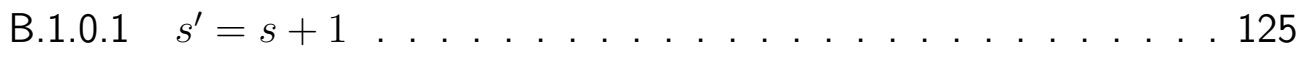

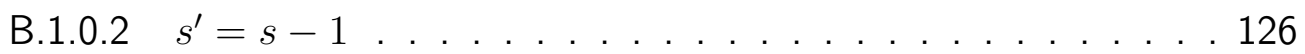

B.2 Cálculo dos elementos de matriz de $f_{m \uparrow}^{\dagger} f_{N \downarrow} \ldots \ldots . \ldots . \ldots . \ldots 127$

B.3 Cálculo dos lementos de matriz de $f_{0 \uparrow}^{\dagger} f_{n \downarrow} \ldots \ldots \ldots$. . . . . . . . . 128

B.3.1 Elementos de $f_{0 \uparrow}^{\dagger} f_{n \downarrow}$ entre estados com $s=s^{\prime}$ e $s_{z}^{\prime} \neq s_{z} \ldots \ldots . . . .128$

B.3.1.1 East-East . . . . . . . . . . . . . . . . . . . . . . . . 129

B.3.2 Elementos de $f_{0 \uparrow}^{\dagger} f_{n \downarrow}$ entre estados com spins diferentes . . . . . . . 131

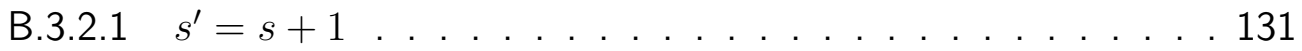

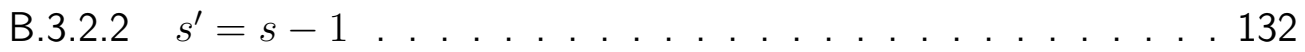

Apêndice C - Condutâncias em nanoestrututas 135

C.1 Condutância universal . . . . . . . . . . . . . . . . . . . . . . . . . 135

C.2 Dispositivo em formato $T \ldots \ldots$. . . . . . . . . . . . . . . 137

C.3 Transístor de um único elétron . . . . . . . . . . . . . . . . . . . . . . . . 138

C.4 Resultados numéricos . . . . . . . . . . . . . . . . . . . . . . . . . . . . . . . . . 139 


\section{Introdução}

Before I came here I was confused about this subject. Having listened to your lecture I am still confused. But on a higher level. Enrico Fermi

Ao longo de décadas, teóricos buscaram explicar o comportamento da resistividade elétrica de metais com a variação da temperatura. Sabemos que na maioria dos metais, o aumento de temperatura é acompanhado por um aumento na resistividade: conforme a temperatura aumenta no metal, cada vez mais vibrações de seus núcleos são excitadas, levando a um espalhamento dos elétrons de condução que resulta em aumento da resistividade. De fato, este tipo de dependência é verificada para altas temperaturas (superiores à temperatura ambiente), até as quais a resistividade é praticamente a resistividade intrínseca do material. A baixas temperaturas, entretanto, verificou-se que a resistividade de muitos metais cresce a medida que a temperatura diminui após passar por um mínimo.

Essa descoberta, que intrigou os físicos por cerca de 30 anos, teve um histórico muito interessante até que, finalmente, Jun Kondo (2) propôs uma explicação para o fenômeno, hoje conhecido como Efeito Kondo.

Em 1934, a observação de um mínimo de resistividade em ouro a baixas temperaturas por Haas, Boer e van den Berg (6) foi um dos primeiros indícios da violação do modelo de resistividade postulado na teoria eletrônica dos metais. Isso levou à suspeita da existência de uma forma de espalhamento anômalo, verificado mesmo quando as vibrações da rede não são suficientes para incitá-lo. Em trabalhos posteriores, o grupo de pesquisadores associou a influência da pureza química e física à posição do mínimo em fios metálicos (7). Na década de 1960, reconheceu-se que o comportamento resistivo a baixas temperaturas estava associado à presença de impurezas magnéticas no metal (8).

Em 1964, van den Berg (8) mostrou que os mínimos de resistividade estariam correlacionados com número de impurezas presentes no metal hospedeiro. No mesmo ano, o físico Jun 
Kondo foi capaz de elucidar a natureza desse fenômeno tal como hoje conhecemos: a presença de impurezas, defeitos ou vacâncias em metais hospedeiros contribui para a resitividade residual a baixas temperaturas (2). A figura 1.1 mostra o comportamento resistivo ou condutivo de alguns materiais, comparando-os com sistemas em que o efeito Kondo pode manifestar-se.
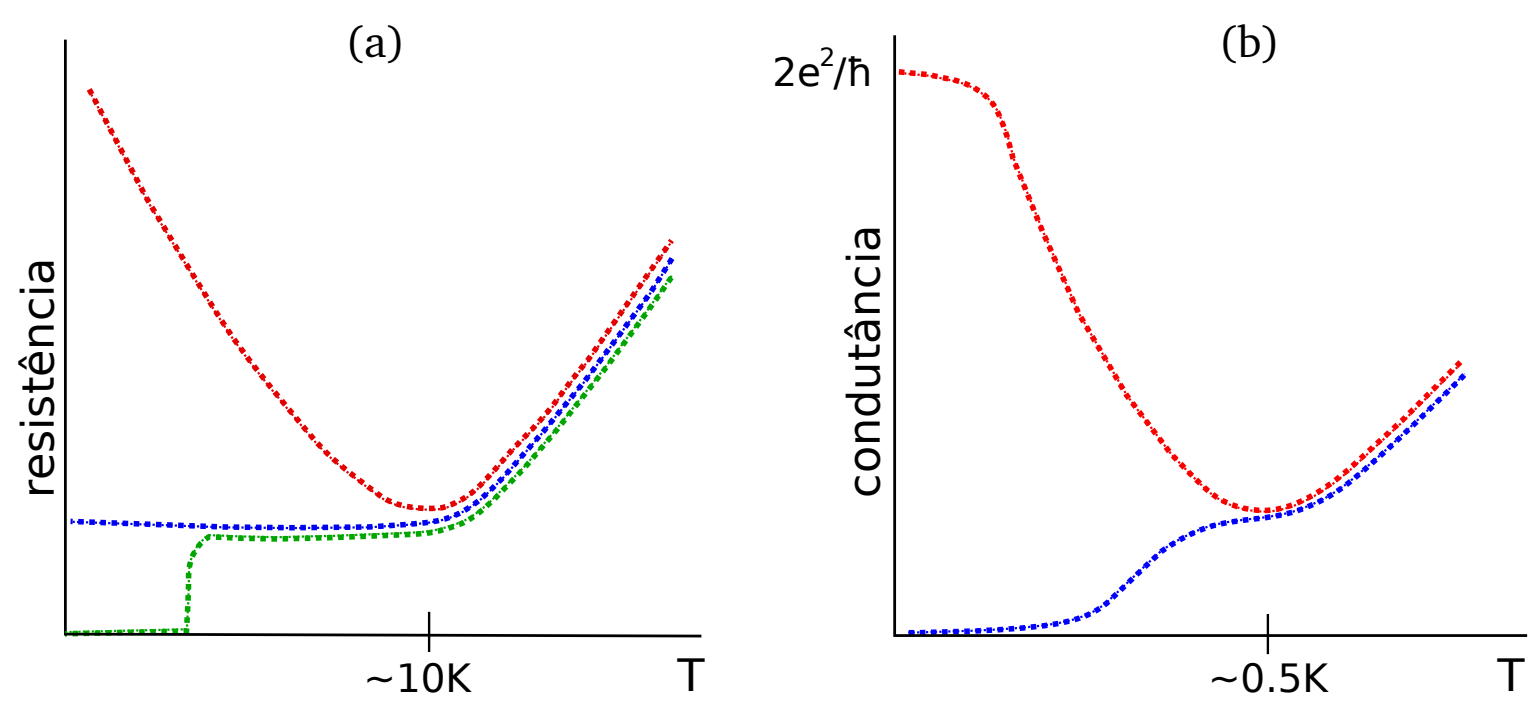

Figura 1.1 - Comportamento resistivo de materiais metálicos. a) A medida que a temperatura no metal é reduzida, sua resistência diminui até alcançar um valor residual (azul). Certos materiais metálicos se tornam supercondutores a partir de uma temperatura crítica (verde). Em ligas metálicas, tal como $\mathrm{CoCu}$, a presença de impurezas dá origem ao Efeito Kondo, fazendo com que a resistência aumente com a redução da temperatura (vermelho). b) Pontos quânticos podem ser criados artificialmente em semicondutores dispondo um spin localizado entre fios metálicos. Sistemas dessa natureza permitem controlar o número de elétrons. Se esse número for ímpar, a condutância medida entre os dois fios aumenta a baixas temperaturas devido ao efeito Kondo (vermelho). Este comportamento não é verificado para um número par de elétrons, já que a condutância decresce a baixas temperaturas (azul). Fonte: KOUWENHOVEN, L. P. (1).

Utilizando a segunda aproximação de Born, Kondo calculou a probabilidade de espalhamento dos elétrons de condução devido à perturbação causada por impurezas e obteve um modelo para a resitividade com um termo logarítmico $\log (T)$ divergente para $T \rightarrow 0$. Ainda no mesmo trabalho, Kondo mostrou que o mínimo observado em metais no regime de baixas temperaturas ocorre quando interação de troca entre o spin da impureza e os elétrons de condução é antiferromagnética. Desde então, o efeito de impurezas magnéticas na resistência de metais - que no regime de baixas temperaturas apresenta um mínimo - é conhecido como Efeito Kondo. 


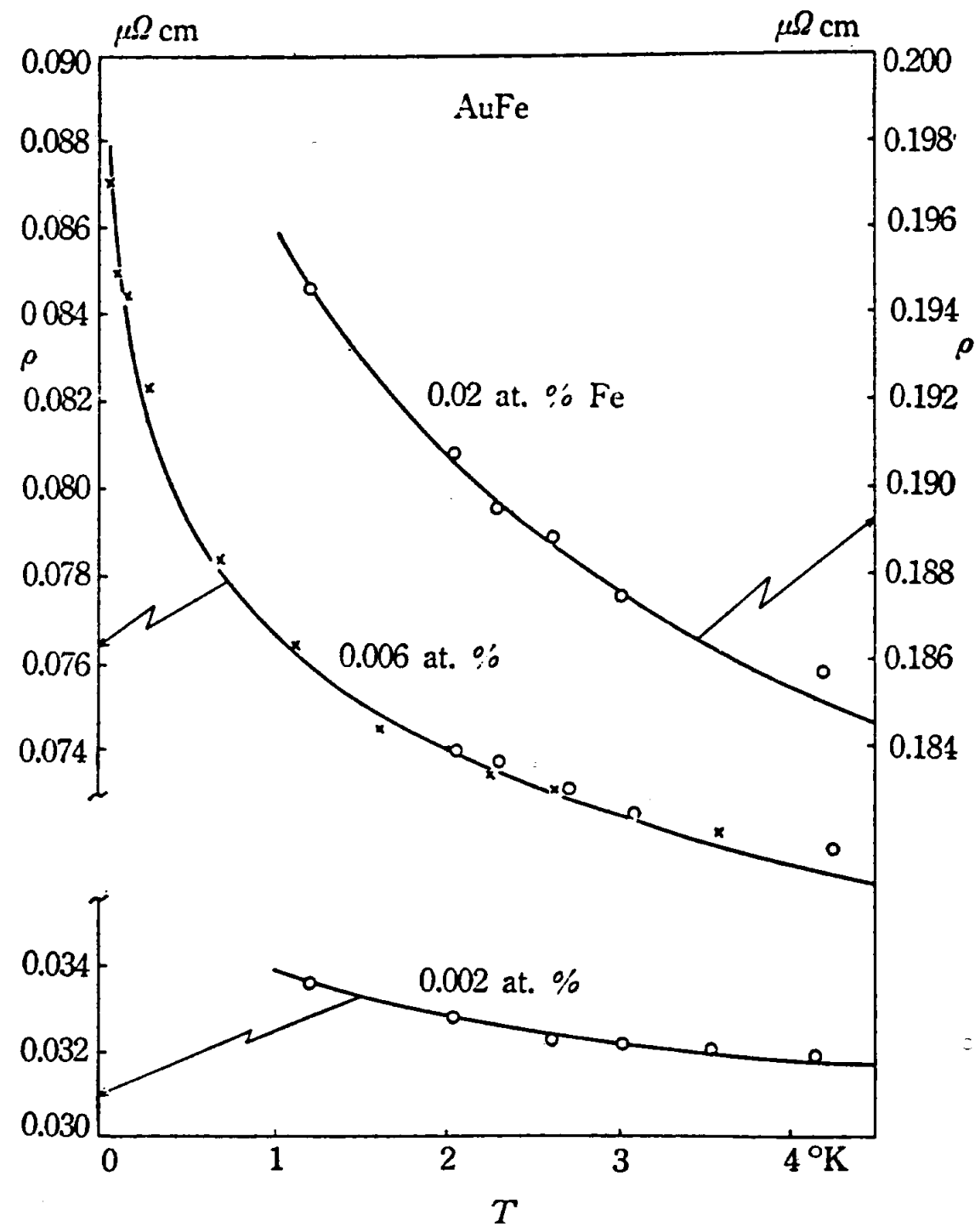

Figura 1.2 - Resistividade $\rho$ em função da temperatura $T$. Comparação da curva experimental $\rho-T$ de ligas diluídas de $A u F e$ com os resultados do modelo de Kondo em seu trabalho de 1964. Fonte: KONDO, J. (2).

Até aquele momento, o modelo de Kondo fornecia a explicação teórica da origem física do fenômeno com resultados compatíveis com estudos experimentais - veja a Figura 1.2. Contudo, ainda era necessário encontrar uma solução isenta de divergêcias. Diversos métodos foram propostos na tentativa de superar tal dificuldade. Phillip Anderson desenvolveu um método de Grupo de Renormalização com o objetivo de eliminar energias altas em favor de uma interação efetiva $J_{\text {eff }}$ entre a impureza e a banda de condução. Conhecido como "Poor Man's Scaling"(9), o método perturbativo de Anderson era inválido para $J$ tendendo a valores grandes. Assim, uma vez que $J_{\text {eff }}$ cresce a medida que as energias baixam, o método se tornava inescapavelmente impreciso depois de algumas rodadas do processo eliminatório. 
a)

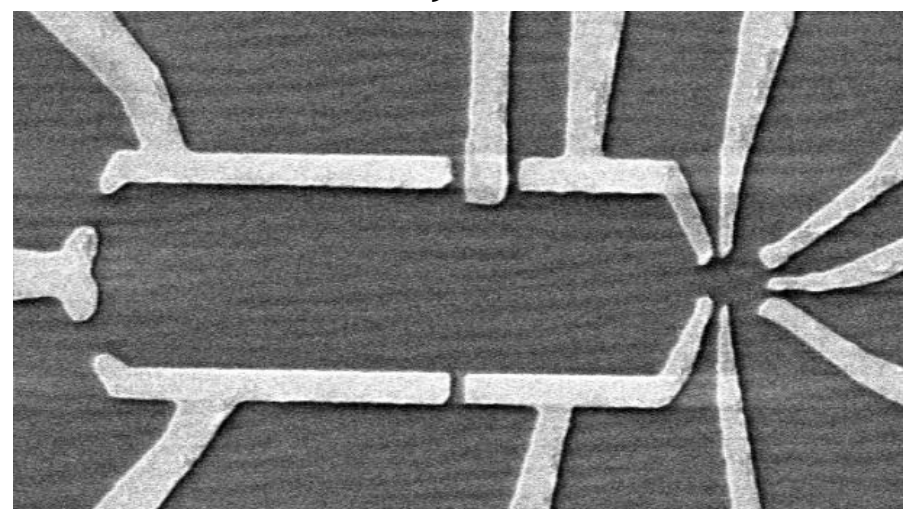

b)

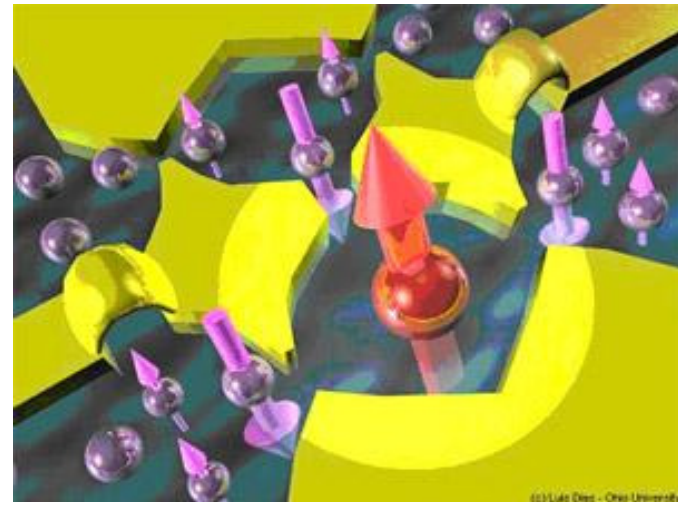

Figura 1.3 - Ilustração de pontos quânticos. a) Esses dispositivos de escala nanomêtrica consistem de eletrodos usualmente construídos por técnicas avançadas de epitaxia em nanocristais, por deposição iônica ou por litografia. Aplicando voltagens aos eletrodos metálicos é possível confinar elétrons em uma pequena região, formando pontos quânticos. Fonte: GOLDHABER-GORDON, D. (3). b) Um segundo exemplo, desta vez constituído por dois pontos quânticos. Pontos quânticos duplos são formados conectando dois pontos quânticos. Um efeito interessante decorre de pontos quânticos duplos: um dos pontos está no estado de Kondo, de modo que o spin do elétron confinado é compensado pelo spin dos elétrons ao redor. Fonte: ScienceDaily (4).

Na década de 70, Kenneth Wilson (10) utilizou o problema Kondo como base para o desenvolvimento do Grupo de Renormalização Numérico (NRG). A técnica descrita por Wilson no trabalho que the rendeu o Nobel obteve resultados promissores no cálculo de observáveis físicos, tais como a susceptibilidade magnética e o calor específico, em todo o limite de temperaturas de interesse. Assim, a divergência do modelo de Kondo foi resolvida satisfatoriamente, já que as propriedades físicas derivadas via NRG são compatíveis com o comportamento esperado quando a temperatura vai a zero. Mais tarde, Krishna-murthy (11) consagrou o sucesso do método na solução do modelo de Anderson com uma impureza (12) .

Embora o problema Kondo seja bem consolidado, ele continua sendo vastamente estudado e tem contribuído sobremaneira à compreensão de propriedades eletrônicas de uma série de materiais, tais como férmions pesados e supercondutores a altas temperaturas. Além disso, o problema se torna inspirador para o teste de novas ferramentas analíticas e numéricas em problemas de muitos corpos.

Com o advento da microscopia de tunelamento (Scanning tunnel microscopy - STM) foi possível estudar o efeito Kondo em experimentos de manipulação atômica e imageamento, com principais resultados em 1998 (13, 14). Atualmente, o efeito Kondo vem sendo explorado em pontos quânticos (15), onde é possível controlá-lo experimentalmente (16-21) - veja a figura 1.3. Há grande interesse da indústria tecnológica em pesquisas com pontos quânticos, pois esses dispositivos são essenciais à fabricação das novas gerações de chips. 
Desse modo, o problema Kondo ainda é bastante atrativo às pesquisas em Matéria Condensada. Em particular, uma questão ainda aberta diz respeito à chamada nuvem Kondo. A nuvem Kondo corresponde à região dinamicamente polarizada que se forma ao redor da impureza devido às correlações eletrônicas e ao forte acoplamento da impureza com os elétrons metálicos a baixas temperaturas. Para um sistema isotrópico infinito, a nuvem poderia ser aproximada por uma esfera de raio $R_{K}$, como mostra a figura 1.4 .

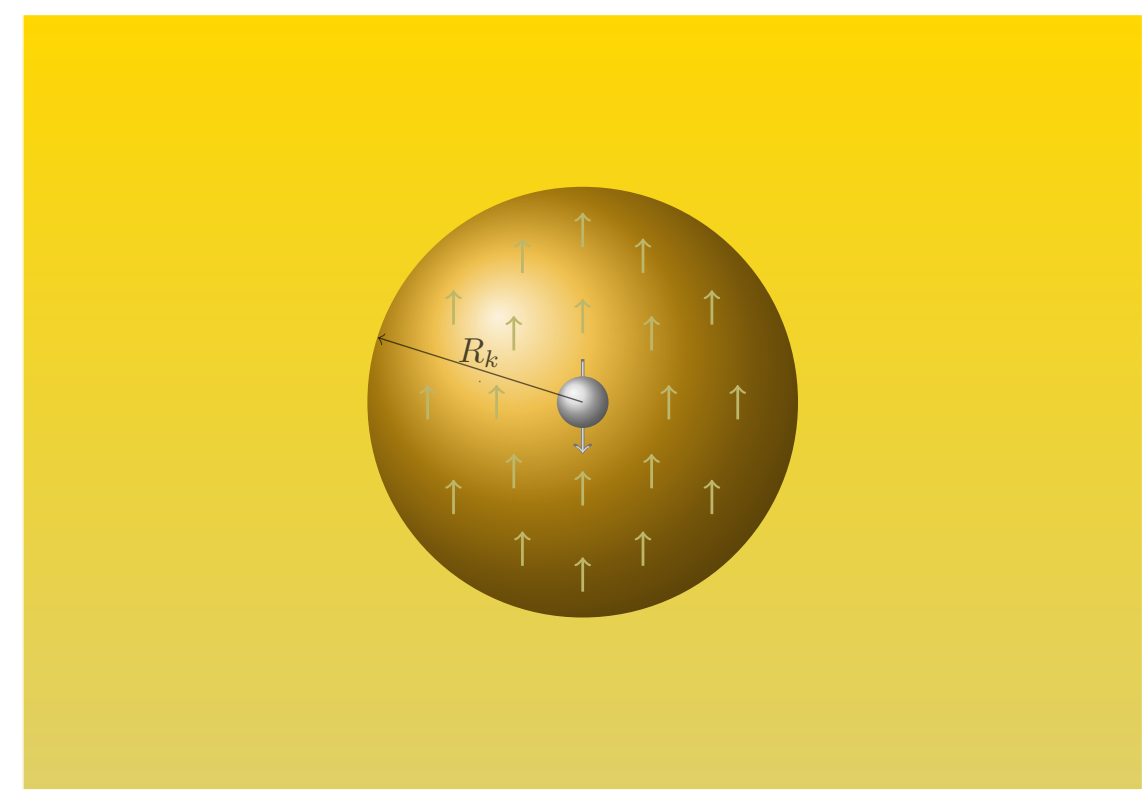

Figura 1.4 - Nuvem Kondo em um sistema metálico infinito e isotrópico com a impureza localizada na origem. Fonte: elaborada pela autora.

A primeira estimativa para o raio da nuvem Kondo concluiu que esse tamanho estaria relacionado ao comprimento de coerência Kondo $\xi_{K}=\hbar v_{F} / k_{B} T_{K}$. Para o valores típicos de temperatura Kondo $T_{K} \sim 1 \mathrm{~K}$ e velocidade de Fermi $v_{F}$ observados em metais, o raio seria encontrado na escala mesoscópica $\operatorname{com} \xi_{K} \sim 1 \mu \mathrm{m}$ - o que contradiria a manifestação do efeito Kondo em sistemas nanoscópicos.

Um dos principais desafios remanescentes envolvendo o efeito Kondo consiste em medir e controlar a nuvem Kondo. Igualmente importantes são o estudo da formação e evolução temporal dos estados quânticos em sistemas de impurezas e da interação entre momentos magnéticos localizados. Há ainda muito a investigar em teorias e experimentos, lançando mão de ferramentas tecnológicas que abrangem desde métodos numéricos avançados até instrumentos ultra-precisos.

Até o presente, diversos trabalhos tentaram estimar o raio da nuvem $R_{K}$ através de ambas abordagens teóricas e experimentais (22-26). Entretanto, discrepâncias entre os resultados 
impediram que se chegasse a um valor $R_{K}$ definitivo. Uma possível explicação para tais inconclusões advém da ideia de que o raio da nuvem Kondo é o mesmo, independente da geometria.

Com o objetivo de verificar se há dependência geométrica na formação da nuvem Kondo, propomos um método para estimar $R_{K}$ em uma liga metática diluída (um metal hospedeiro com baixa concentração de impurezas) com geometria especial. Em particular, vamos considerar um metal semi-infinito na direção $z$ com a impureza localizada a uma distância $d$ do plano que delimita o metal. Então, estudaremos a taxa de relaxação magnética $1 / T_{1}(5,27,28)$ de uma ponta de prova como função da temperatura $T$ e da sua distância $R$ à impureza.

Experimentalmente nossa abordagem é semelhante à reportada por Boyce e Slichter $(5,5)$ em 1964 em medidas de ressonância magnética. Nesse trabalho, o grupo mediu o deslocamento Knight de impurezas de ferro em um hospedeiro de cobre como função da temperatura, da distância da ponta de prova às impurezas e da concentração destas. Como resultado, eles obtiveram a seguinte relação para a polarização:

$$
\chi(r, T)=f(r) g(T)
$$

Além de verificarem a expressão 1.1 para o deslocamento Knight, Boyce e Slichter mostraram que a relaxação do sistema é muito pouco afetada pela distância e, assim, concluíram que não seria possível precisar qual a distância $r$ associada ao tamanho $R_{K}$ da nuvem Kondo. Contudo, uma vez que o sinal medido pela ponta de prova física decai rapidamente com a distância, esses resultados valem apenas nas proximidades das impurezas. Na prática, tais resultados não valem na escala mesoscópica, onde supostamente estaria o raio da nuvem Kondo. Caso pudéssemos simular a medida da taxa de relaxação para distâncias suficientemente grandes (sem limitações experimentais),s gostaríamos de verificar se $1 / T_{1}$ é um procedimento válido para estimar o raio da nuvem Kondo.

Nesta dissertação, propomos uma abordagem inicial para tratar esse problema computacionalmente. Em nossa formulação, o Hamiltoniano que representa a ponta de prova, denotado $H_{\text {probe }}$, é responsável pela transição entre estados de spin dos elétrons metálicos e da ponta de prova. Como consequência, a taxa de relaxação $1 / T_{1}$ é uma combinação de três termos: $\left(1 / T_{1}\right)_{s c a}$, $\left(1 / T_{1}\right)_{v e t}$ e $\left(1 / T_{1}\right)_{\text {mat }}$. A contribuição $\left(1 / T_{1}\right)_{s c a}$, que chamamos escalar, corresponde a uma constante de fundo, independente da temperatura. A componente $\left(1 / T_{1}\right)_{v e t}$, ou vetorial, diz respeito à interferência entre os estados metálicos acoplados à impureza e os estados de condução da ponta de prova. Por fim, o termo que chamamos matricial, denotado $\left(1 / T_{1}\right)_{\text {mat }}$, é devido somente aos estados de condução do sistema não perturbado, ou seja, 
aos estados de ondas $s$ dos elétrons de condução ao redor da impureza.

Uma vez que nosso interesse é voltado às medidas de $1 / T_{1}$ na região experimentalmente inacessível, vamos começar tratando de distâncias $R$ e $d$ tais que a ponta de prova e a impureza encontram-se longe da superfície e interagem a longo alcance. Nessa situação, a taxa $\left(1 / T_{1}\right)_{\text {mat }}$ pode ser desprezada, pois decai rapidamente quando $R$ aumenta. Assim, vamos analisar o comportamento da taxa $\left(1 / T_{1}\right)_{v e t}$ da contribuição vetorial.

O cálculo da taxa $1 / T_{1}$ requer essencialmente que diagonalizemos o Hamiltoniano do sistema não-perturbado, descrito pelo Hamiltoniano de Anderson $H_{A}$. Essa tarefa é realizada através do método de Grupo de Renormalização Numérico (NRG), que nos permite obter o espectro de $H_{A}$, seus autovalores e, também, computar elementos de matriz reduzidos (conhecidos como invariantes).

Os desenvolvimentos que propomos aqui para o cálculo da taxa de relaxação podem ser generalizados para outras geometrias, incluindo também os efeitos de borda nas proximidades das superfícies metálicas. Em trabalhos futuros, estaremos interessados na taxa de relaxação associada à contribuição matricial, que tornam-se importantes quando reduzimos $R$ progressivamente e onde os efeitos de borda não podem ser desprezados. Nesse sentido, o presente trabalho representa uma primeira contribuição para a investigação da influência da geometria à formação da nuvem Kondo. 


\section{O sistema quântico}

More is different.

P.W. Anderson

Quando uma impureza magnética ( $\mathrm{Fe}$, por exemplo) se encontra em um ambiente metálico não magnético ( $\mathrm{Au}$, por exemplo), surge uma interação antiferromagética entre os elétrons do metal e os da impureza. Em torno desta, no regime de baixas temperaturas, forma-se uma região dinamicamente polarizada, tradicionalmente chamada de nuvem de Kondo.

A medida do tamanho da nuvem, que em primeira instância seria associada ao seu raio $R_{K}$, representa uma interessante questão que permanece indefinida no problema Kondo. Do modelo de Kondo para a resistividade em ligas com impurezas à atual fase de estudos em pontos quânticos, foram propostos diversos trabalhos buscando estimar teoricamente ou medir experimentalmente o tamanho da nuvem Kondo. Contudo, não há consenso entre os resultados obtidos, de modo que não foi possível encontrar $R_{K}$.

Um aspecto não considerado nos trabalhos precedentes é a geometria do sistema, que acreditamos afetar a formação da nuvem Kondo. Nossa proposta consiste, então, em escolher um sistema de geometria particular e verificar como esta altera alguma propriedade intrinsecamente relacionada à polarização dos spins da nuvem. Nesse sentido, estudaremos a taxa de relaxação magnética de um spin de prova imerso no sistema metálico.

O sistema que investigamos é constituído de duas partes. A primeira, ilustrada na figura 2.1, refere-se ao metal hospedeiro de geometria semi-infinita com uma impureza magnética localizada no eixo $z$ a uma distância $d$ do plano $z=0$. Esse termo é descrito quanticamente pelo Hamiltoniano de Anderson.

A segunda parte do nosso sistema constitui a ponta de prova, que tratamos como uma perturbação do sistema original. Consideramos que a ponta de prova é fixa na posição $\vec{R}$, a uma distância $R$ da impureza. Mostraremos como o Hamiltoniano da ponta de prova $H_{\text {probe }}$ é ultilizado no cálculo da taxa de relaxação magnética $1 / T_{1}$. 


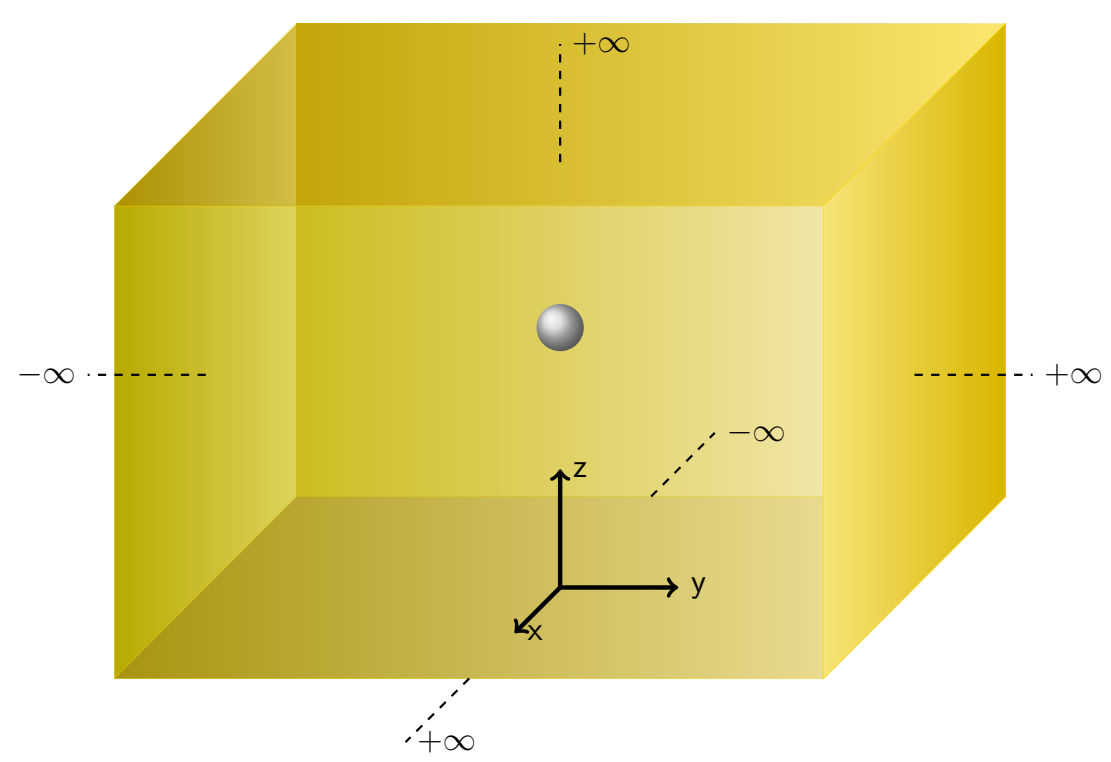

Figura 2.1 - Metal hospedeiro semi-infinito na direção $z$ com a impureza localizada à distância $\vec{d}$ do plano limitante $z=0$. Fonte: elaborada pela autora.

Fisicamente, nossa abordagem pressupõe que a relaxação da ponta de prova seja sensível à nuvem Kondo e dependa da distância $R$ e da temperatura $T$. Caso contrário, esperamos observar um comportamento equivalente ao do metal hospedeiro na ausência de impurezas.

No regime de baixas temperaturas, a nuvem Kondo pode se formar de duas formas distintas. No caso em que $R_{k}<d$, a nuvem consegue se formar dentro do material como se não existisse superfície - como ilustramos na figura 2.2. Neste caso, espera-se que o raio da nuvem seja dado por $\xi_{K}=\hbar v_{F} / k_{B} T_{K}(22)$, .

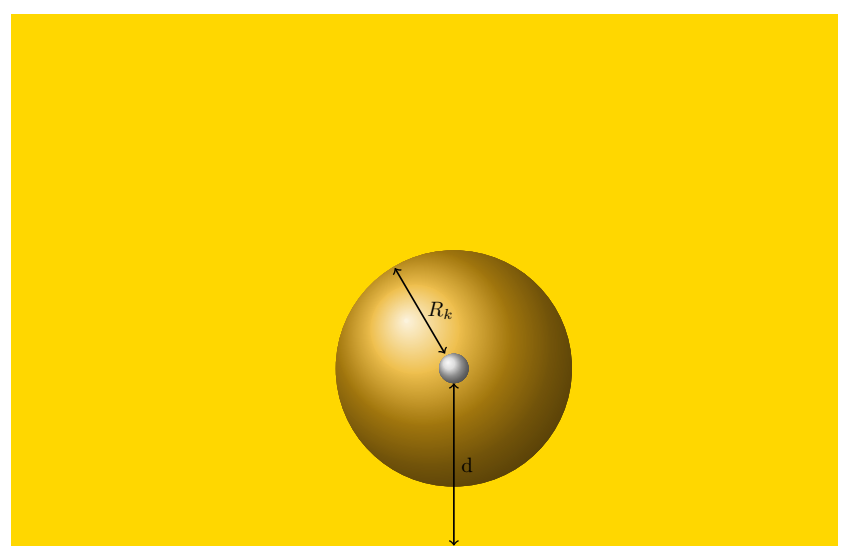

Figura 2.2 - Para $R_{K}<d$ a nuvem Kondo não há truncamento pela superfície, de modo que o comportamento esperado é o de uma nuvem com raio inversamente proporcional a $T_{K}$, como descrito em (5). Fonte: elaborada pela autora. 
Por outro lado, se $R_{k}>d$, de modo que a nuvem é parcialmente truncada pela superfície - como ilustrado na figura 2.3 - o tamanho da nuvem ficaria dependente da geometria do sistema.

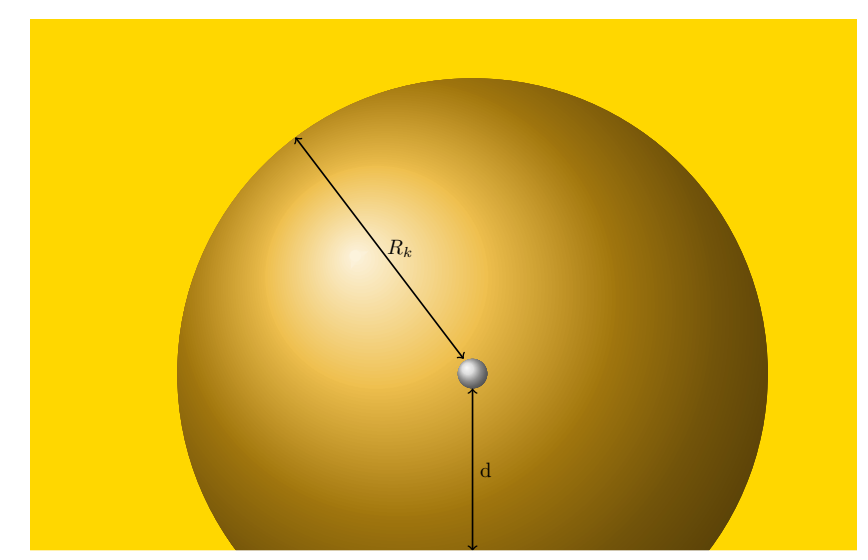

Figura 2.3 - Para $R_{K}>d$ a nuvem Kondo é truncada pela superfície e seu tamanho dependeria da geometria do sistema. Fonte: elaborada pela autora.

A seguir, mostraremos como cada uma das partes envolvidas no nosso sistema - o metal hospedeiro com a impureza e a ponta de prova - são representados quanticamente pelos Hamiltonianos de Anderson $H_{A}$ e $H_{\text {probe }}$.

\subsection{O Modelo de Anderson de uma impureza}

O Modelo de Anderson (12) é um modelo de Hamiltoniano usado para descrever quanticamente sistemas metálicos com impurezas magnéticas diluídas. O Hamiltoniano de Anderson $H_{A}$ engloba três termos, referentes à banda de condução do metal hospedeiro com elétrons não-interagentes, ao orbital da impureza localizado e à hibridização:

$$
H_{A}=H_{\text {cond }}+H_{d}+H_{\text {int }} .
$$

A banda de condução, de largura energética $2 D$, tem densidade de estados dada por:

$$
\rho=\frac{N}{2 D}
$$


e é definida pelo Hamiltoniano $H_{\text {cond }}$ :

$$
H_{\text {cond }}=\sum_{\mathbf{k} \mu} \varepsilon_{\mathbf{k}} c_{\mathbf{k} \mu}^{\dagger} c_{\mathbf{k} \mu}
$$

onde $\varepsilon_{\mathbf{k}}$ são as energias dos elétrons da banda com momento $\mathbf{k}$, medidas a partir do nível de Fermi, e $c_{\mathbf{k} \mu}^{\dagger}\left(c_{\mathbf{k} \mu}\right)$ são os operadores de criação (aniquilação) de elétrons com spin $\mu= \pm \frac{1}{2}$.

Em um metal real, a descrição dos elétrons livres da banda de condução pela eq. (2.3) corresponde a estados de onda $s$ ou $p$.

A banda de condução é isotrópica $\left(\varepsilon_{\mathbf{k}}=\varepsilon_{k}\right)$ e estende-se de $-D$ a $D$ em torno de $k_{F}$. Consideramos que a relação de dispersão é aproximadamente linear, ou seja,

$$
\varepsilon=\frac{v_{F}}{D}\left(k-k_{F}\right)
$$

onde $v_{F}$ é a velocidade associada ao nível de Fermi e $k_{F}$ é o momento de Fermi.

A aproximação linear em 2.4 tem como justificativa nosso interesse em energias $\left(k_{B} T \ll\right.$ $\varepsilon_{F}$ ) bem menores do que a energia de Fermi. Veja a figura 2.4 .

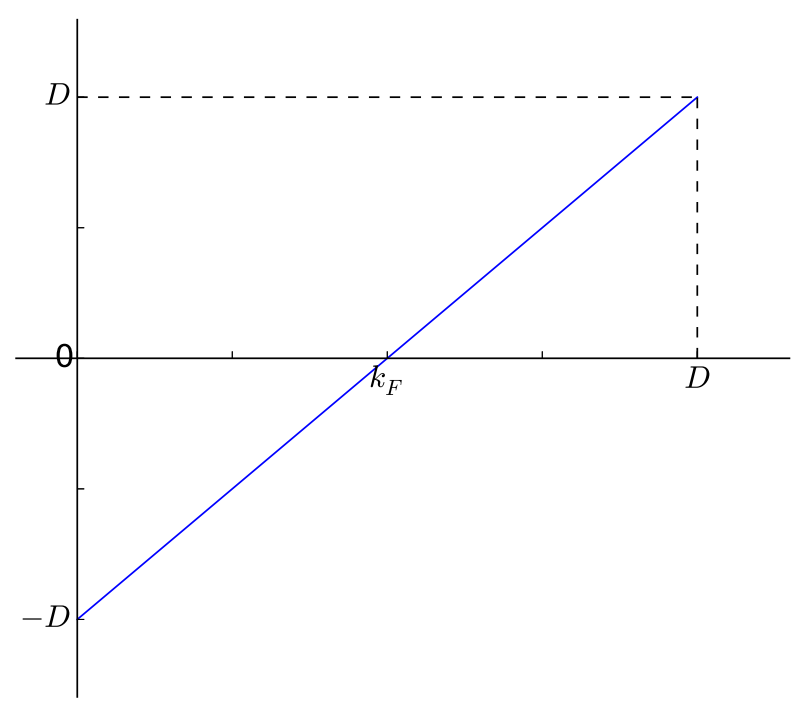

Figura 2.4 - A relação de dispersão para as energias da banda de condução no intervalo $[-D, D]$ é aproximada por uma função linear, simétrica em torno do momento de Fermi $k_{F}$. Fonte: elaborada pela autora. 
A impureza é descrita pelo Hamiltoniano $H_{d}$ :

$$
H_{d}=\sum_{\mu} \varepsilon_{d} c_{d \mu}^{\dagger} c_{d \mu}+U n_{d \uparrow} n_{d \downarrow}
$$

onde $\varepsilon_{d}$ denota a energia da impureza no orbital $d$ e $U$ é a repulsão Coulombiana no nível duplamente ocupado.

O acoplamento (hibridização) é responsável pela troca de elétrons entre a banda de condução e o nível localizado na impureza. O Hamiltoniano correspondente, $H_{\text {int }}$, é

$$
H_{i n t}=\sqrt{\frac{\Gamma}{\pi}} \sum_{\mathbf{k} \mu}\left(e^{i \mathbf{k} . \mathbf{d}} c_{\mathbf{k} \mu}^{\dagger} c_{d \mu}+H . c .\right),
$$

onde $d$ é posição da impureza no metal hospedeiro.

As equações (2.3), (2.5) e (2.6) definem o modelo de Anderson para descrever um metal hospedeiro com uma impureza, também conhecido como Single Impurity Anderson Model (SIAM). Em particular, o termo de interação $H_{\text {int }}$ descreve a repulsão - de largura finita $\Gamma$ entre os elétros da impureza localizada e os níveis da banda de condução.

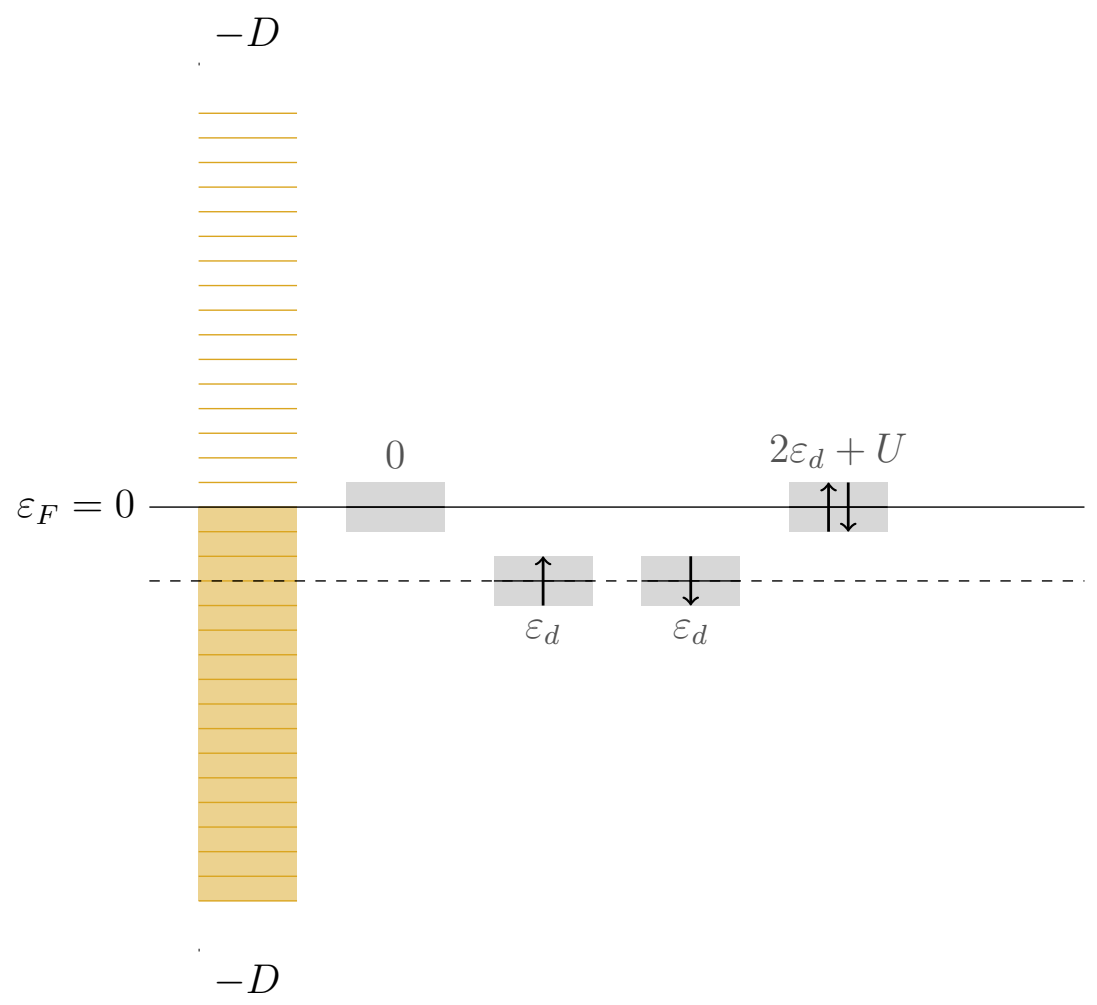

Figura 2.5 - Representação dos níveis de energia no Modelo de Anderson simétrico. Nesta situação $\varepsilon_{d}=-U / 2$, e o estado da impureza pode assumir quatro configurações: vazia $\left(n_{d}=0\right)$, semi ocupada com um elétron com spin up ou down $\left(n_{d}=1\right)$ e cheia com dois elétrons com spins opostos $\left(n_{d}=2\right)$. Fonte: elaborada pela autora. 
Os parâmetros energéticos $U, \varepsilon_{d}$ e $\Gamma$ determinam alguns limites do modelo de Anderson. Por exemplo, quando $\varepsilon_{d}=-U / 2$ ocorre a degenerescência dos estados vazio e totalmente cheio da impureza, como ilustra a figura 2.5. Nesta situação, chamada de modelo de Anderson simétrico, o Hamiltoniano correspondente,

$$
H_{A}^{S}=\sum_{k} \varepsilon_{k} c_{k}^{\dagger} c_{k}+\sqrt{\frac{\Gamma}{\pi}} \sum_{k}\left(c_{k}^{\dagger} c_{d}+H . c .\right)-\frac{U}{2}\left(c_{d \uparrow}^{\dagger} c_{d \uparrow}-c_{d \downarrow}^{\dagger} c_{d \downarrow}\right)^{2},
$$

torna-se invariante pelas trocas $c_{k}^{\dagger} \leftrightarrow c_{-k}$ e $-c_{d}^{\dagger} \leftrightarrow c_{d}$, dando origem à simetria partícula buraco.

a)

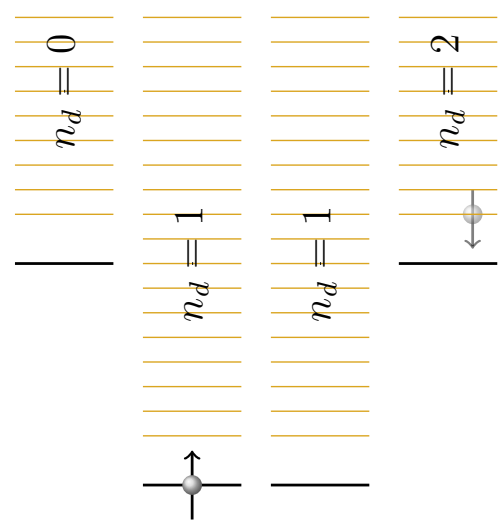

b)

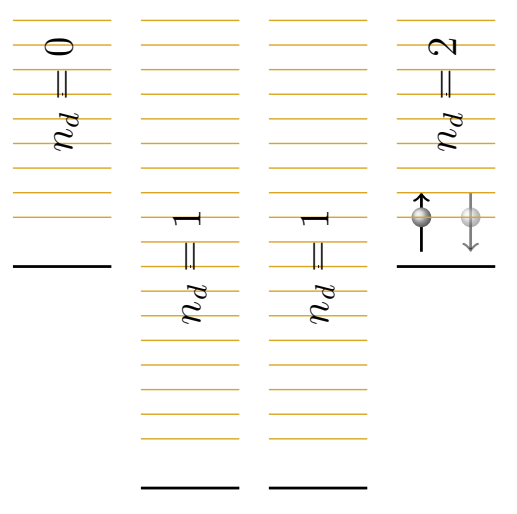

c)

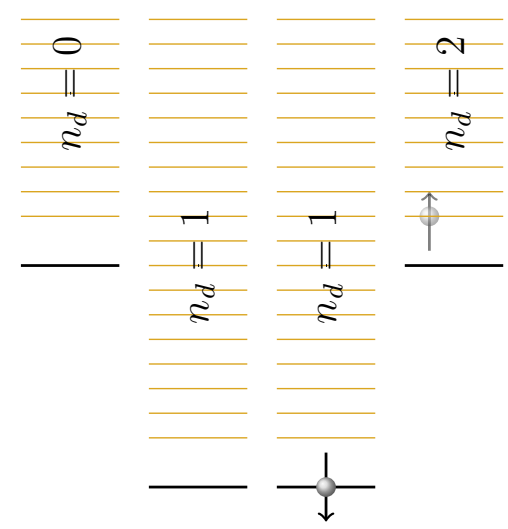

Figura 2.6 - Transições virtuais da impureza no regime de acoplamento forte para o Modelo de Anderson simétrico. Nívels da banda de condução são representados em dourado, enquanto a impureza é ilustrada em prateado. a) Estado inicial da impureza no nível $\varepsilon_{d}$ com spin up. b) Uma vez que adicionar um elétron não é energeticamente possível por conta do valor de $U$, o elétron up da impureza ocupa um estado virtual e então é substituído por um elétron do metal. c) Da transição virtual resulta o flip (inversão) de spin da impureza. Fonte: elaborada pela autora.

Quando a interação repulsiva é suficientemente grande, isto é, para $U \gg \Gamma$, o nível da impureza $\varepsilon_{d}$ não pode ser encontrado com dois elétrons de spins opostos. Forma-se um spin localizado no nível da impureza e o sistema entra no regime de acoplamento antiferromagnético, no qual são permitidas transições virtuais entre os estados da banda de condução e da impureza. Nessa situação, o Hamiltoniano (2.1) reproduz exatamente o modelo de impurezas estudado por Kondo e pode ser reescrito na forma (11):

$$
H_{K}=\sum_{k} \varepsilon_{k} c_{k}^{\dagger} c_{k}+2 J \vec{S} \cdot \sum_{k, q, \mu, \nu} c_{k \mu}^{\dagger} \vec{\sigma}_{\mu \nu} c_{q \nu}
$$

onde $\vec{S}$ é o spin da impureza, $\vec{\sigma}$ é o vetor composto pelas matrizes de Pauli e $J>0$ é a 
constante de acoplamento calculada como (29)

$$
J=\frac{2 \Gamma}{\rho \pi} \frac{U}{\left|\varepsilon_{d}\right|\left(\varepsilon_{d}+U\right)} .
$$

Por outro lado, no limite não-interagente $U \ll \Gamma$, a impureza se amalgama com a banda de condução e apenas aumenta a densidade de estados desta última. Esse limite tem pouco interesse.

Ambos os Hamiltonianos (2.1) e (2.8) permitem descrever sistemas Kondo, embutindo a física do acoplamento entre a impureza e os elétrons de condução. Contudo, há dificuldades em resolvê-los analiticamente, já que as somas sobre os momenta $k$ estendem-se por toda a banda de condução de uma forma contínua. Para superar essa dificuldade, recorremos ao método do Grupo de Renormalização Numérico (NRG), que nos permite diagonalizar numericamente - Hamiltoniano (2.1). Antes de derivar a expressão do Hamiltoniano passível de tratamento numérico, precisamos definir a o Hamiltoniano $H_{\text {probe }}$ descrevendo a interação do sistema não perturbado (metal hospedeiro e a impureza magnética) com a ponta de prova.

\subsection{A ponta de prova}

Voltando ao Hamiltoniano (2.8), observamos que o termo proporcional a $J$ em $H_{K}$ tem dois efeitos, respectivos à parcela longitudinal $(z)$ e às transversais ( $x$ e $y$ ). A contribuição devida a $\sigma_{z}$ polariza antiferromagneticamente os elétrons ao redor da impureza. As parcelas proporcionais a $\sigma_{x}$ e $\sigma_{y}$ dão origem a processos de espalhamento que invertem, simultaneamente, os spins da impureza e do elétron de condução espalhado. Assim, este último termo faz com que o spin da impureza sofra inversões a uma taxa $\Omega$ que depende da temperatura e da polarização dos elétrons em torno da impureza.

A altas temperaturas a polarização não consegue compensar a taxa $\Omega$. Por exemplo, na inversão do spin da impureza entre os estados $\mu=\uparrow \mathrm{e}-\mu=\downarrow$, a polarização deve trocar de sinal, passando de positiva a negativa. Contudo, isto requer que os spins de muitos elétrons de condução sejam invertidos. Na prática, estes últimos ficam eficazmente desacoplados da impureza de forma que a taxa de relaxação da impureza pode ser calculada como se não houvesse polarização.

Um efeito diferente ocorre ao resfriar o metal, pois a taxa de relaxação tende a diminuir, ou seja, porque o tempo médio de relaxação tende a aumentar. A medida em que o tempo médio 
de relaxação cresce, este aos poucos se aproxima do tempo médio de inversão da polarização. Na temperatura de Kondo $T_{K}$, as taxas de relaxação da impureza e de inversão da polarização se tornam comparáveis e a polarização passa a exercer um efeito viscoso sobre a relaxação da impureza, originando, portanto, um efeito de ressonância. Dada a degenerescência dos estados $\uparrow e \downarrow$ da impureza, a frequência de ressonância é nula. A largura da ressonância é determinada pela taxa de inversão da polarização e assim é da ordem de $\hbar / k_{B} T_{K}$, onde $k_{B}$ é a constante de Boltzman. Para valores típicos de temperatura $T_{K} \sim 1 \mathrm{~K}$ a largura de ressonância é aproximadamente $0.1 \mathrm{meV}$.

A inversão da polarização pode ser medida por ressonância magnética $(5,27)$. Para tanto, é preciso medir a relaxação magnética de um spin de prova posicionado a uma distância $R$ da impureza. Em torno desta, a baixas temperaturas, forma-se a nuvem Kondo com extensão espacial $R_{K}$. Desse modo, esperamos que quando a ponta estiver no interior da região polarizada, ou seja, $R<R_{k}$, ela seja sensível à dinâmica da nuvem.

A perturbação (28) da ponta de prova no sistema não perturbado (metal e impureza) é descrita pelo Hamiltoniano $H_{\text {probe }}$, definido por

$$
H_{\text {probe }}=-A\left[\Psi_{\uparrow}^{\dagger}(\vec{R}) \Psi_{\downarrow}(\vec{R}) I_{-}+\Psi_{\downarrow}^{\dagger}(\vec{R}) \Psi_{\uparrow}(\vec{R}) I_{+}\right],
$$

onde $A$ é a interação de troca efetiva constante entre os elétrons de condução e a ponta; $\Psi_{\mu}(\vec{R})=\sum_{\vec{k}} e^{i \vec{k} \vec{R}} c_{k \mu}$ é o operador de campo responsável pelo aniquilamento de um elétron da ponta num sítio $\vec{R} ; I_{-(+)}$é o operador de spin de abaixamento (levantamento), representando o estado magnético da ponta de prova.

Na equação (2.10) a ausência do termo longitudinal $\Psi_{\mu}(R)^{\dagger} \Psi_{\mu}(R) I_{z}$ deve-se ao fato de que o mesmo não contribui para a troca de spin e, por conseguinte, não afeta a taxa de relaxação.

A seguir, exploraremos os conjuntos de transformações de bases quânticas que nos permitem escrever o Hamiltoniano do na eq.(2.1) de forma computável.

\subsection{Base contínua}

Nosso sistema é caracterizado essencialmente pelos Hamiltonianos $H_{A}$ e $H_{\text {probe }}$, das equações (2.1) e (2.10), os quais carregam as dependências espaciais da impureza e da ponta de prova. Desse modo, antes de iniciar o procedimento do NRG, é conveniente fazer uma mu- 
dança de base, de forma que o Hamiltoniano $H$ seja escrito apenas em termos de operadores embutindo tais dependências.

Introduzimos a base contínua de operadores $c_{\varepsilon}$ e $d_{\varepsilon}$ que representam ondas esféricas em torno de $\vec{d}$ e $\vec{R}$, respectivamente. Como tais operadores não são ortogonais, é preciso construir uma nova base $\left\{c_{\varepsilon}, \bar{c}_{\varepsilon}\right\}$ através do método de Gram-Schmidt. Fazendo isto, seremos capazes de definir as mudança para a base $\left\{f_{n}\right\}$ do Grupo de Renormalização, na qual $H_{A}$ pode ser numericamente tratado.

Utilizando a relação de dispersão na equação (2.4), definimos a base de operadores $\left\{c_{\varepsilon}, d_{\varepsilon}\right\}$ como combinação dos $c_{k}$ :

$$
c_{\varepsilon \mu}=\frac{1}{\sqrt{\rho}} \sum_{\mathbf{k}} \delta\left(\varepsilon-\varepsilon_{k}\right) e^{i \mathbf{k} . \mathbf{d}} c_{\mathbf{k} \mu},
$$

e

$$
d_{\varepsilon \mu}=\frac{1}{\sqrt{\rho}} \sum_{\mathbf{k}} \delta\left(\varepsilon-\varepsilon_{k}\right) e^{i \mathbf{k} \cdot \mathbf{R}} c_{\mathbf{k} \mu}
$$

onde $\vec{d}$ e $\vec{R}$ são as posições da impureza e da ponta de prova, respectivamente, e $\rho$ é a densidade de estados dada na equação (2.2).

Os operadores $c_{\varepsilon \mu}\left(c_{\varepsilon \mu}^{\dagger}\right)$ e $d_{\varepsilon \mu}\left(d_{\varepsilon \mu}^{\dagger}\right)$ atuam de modo análogo aos $c_{k}\left(c_{k}^{\dagger}\right)$. Os $c_{\varepsilon \mu}$ aniquilam um elétron localizado em $\vec{d}$, com energia $\varepsilon$ e spin $\mu$, no estado de onda $s$ do sistema não perturbado (íon de Anderson), enquanto os $d_{\varepsilon \mu}$ destroem um elétron da ponta de prova na posição $\vec{R}$. Eles obedecem às relações de comutação

$$
\left\{c_{\varepsilon \mu}^{\dagger}, c_{\varepsilon^{\prime} \mu^{\prime}}\right\}=\delta\left(\varepsilon-\varepsilon^{\prime}\right) \delta_{\mu, \mu^{\prime}}
$$

e

$$
\left\{d_{\varepsilon \mu}^{\dagger}, d_{\varepsilon^{\prime} \mu^{\prime}}\right\}=\delta\left(\varepsilon-\varepsilon^{\prime}\right) \delta_{\mu, \mu^{\prime}}
$$

Os operadores $c_{\varepsilon}$ e $d_{\varepsilon}$ não são ortogonais, pois seu comutador é

$$
\left\{c_{\varepsilon \mu}^{\dagger}, d_{\varepsilon^{\prime} \mu^{\prime}}\right\}=\frac{\sin (k|\vec{R}-\vec{d}|)}{k|\vec{R}-\vec{d}|} \delta\left(\varepsilon-\varepsilon^{\prime}\right) \delta_{\mu, \mu^{\prime}} .
$$

Assim, deveremos encontrar uma base de operadores ortogonais entre si para reescrever o Hamiltoniano total do sistema nesta nova base. Para isso, aplicamos o método de GramSchmidt (28) para definir o operador $\bar{c}_{\varepsilon}$ ortogonal a $c_{\varepsilon}$, dado por

$$
\bar{c}_{\varepsilon \mu}=\frac{1}{\sqrt{1-W^{2}}}\left(d_{\varepsilon \mu}-W(\varepsilon, R) c_{\varepsilon \mu}\right),
$$


onde

$$
W(\varepsilon, R)=\frac{\sin (k R)}{k R},
$$

com $R=\mid \vec{R}-\overrightarrow{d \mid}$ sendo a distância entre a impureza e a ponta de prova e

$$
k R=k_{F} R\left(1+\frac{\varepsilon}{D}\right) \text {. }
$$

A equação (2.18) define $k$ como função explícita das energias, sem a aproximação $k=$ $k_{F}$. Somente podemos desprezar o segundo termo entre os parênteses à direita na equação (2.18) quando o produto $k_{F} R \epsilon / D$ for muito menor do que a unidade, porque as funções trigonométricas de $k R$ dependem apenas de $k R \bmod \pi$, que é da ordem da unidade.

Não podemos desprezar o valor efetivo de $k$, pois as oscilações da função $\sin (k|\vec{R}-\vec{d}|)$ têm efeito apreciável mesmo quando seu argumento varia muito pouco, isto é, quando ( $k \ll$ $\left.k_{F}\right)$. Isto ficará mais claro no Capítulo 4, onde mostraremos como a adoção de $k(\varepsilon)$ é importante para o cálculo da taxa de relaxação.

Podemos agora reescrever os Hamiltonianos (2.3) e (2.6) na base $\left\{c_{\varepsilon}, \bar{c}_{\varepsilon}\right\}$. O Hamiltoniano de condução $H_{\text {cond }}$ na eq. (2.3) fica expresso em termos de autoestados que representam ondas $s$ esféricas com centros de simetria situados no íon de Anderson $\left(H_{c}\right)$

$$
H_{c}=\int_{-D}^{D} d \varepsilon \varepsilon c_{\varepsilon \mu}^{\dagger} c_{\varepsilon \mu},
$$

e na ponta de prova $\left(H_{\bar{c}}\right)$

$$
\bar{H}_{c}=\int_{-D}^{D} d \varepsilon \varepsilon \bar{c}_{\varepsilon \mu}^{\dagger} \bar{c}_{\varepsilon \mu}
$$

Segue que o Hamiltoniano da banda de condução recebe ambas as contribuições (2.19) e (2.20), ou seja,

$$
H_{\text {cond }}=\int_{-D}^{D} \varepsilon\left(c_{\varepsilon \mu}^{\dagger} c_{\varepsilon \mu}+\bar{c}_{\varepsilon \mu}^{\dagger} \bar{c}_{\varepsilon \mu}\right) d \varepsilon .
$$

O Hamiltoniano de hibridização fica escrito como:

$$
H_{\text {int }}=\sqrt{\frac{\Gamma}{\pi}} \int_{-D}^{D} d \varepsilon\left(c_{\varepsilon}^{\dagger} c_{d}+c_{d}^{\dagger} c_{\varepsilon}\right) .
$$


Finalmente, o Hamiltoniano da ponta de prova na eq. (2.10) toma a forma

$$
\begin{aligned}
H_{\text {probe }}= & -A\left[\iint_{-D}^{D} d \varepsilon d \varepsilon^{\prime} \sqrt{1-W(\varepsilon, R)} \sqrt{1-W\left(\varepsilon^{\prime}, R\right)} \bar{c}_{\varepsilon \mu}^{\dagger} \bar{c}_{\varepsilon^{\prime} \nu}\right. \\
& +\iint_{-D}^{D} d \varepsilon d \varepsilon^{\prime} \sqrt{1-W(\varepsilon, R)} W\left(\varepsilon^{\prime}, R\right) \bar{c}_{\varepsilon \mu}^{\dagger} c_{\varepsilon^{\prime} \nu} \\
& +\iint_{-D}^{D} d \varepsilon d \varepsilon^{\prime} W(\varepsilon, R) \sqrt{1-W\left(\varepsilon^{\prime}, R\right)} c_{\varepsilon \mu}^{\dagger} \bar{c}_{\varepsilon^{\prime} \nu} \\
& \left.+\iint_{-D}^{D} d \varepsilon d \varepsilon^{\prime} W(\varepsilon, R) W\left(\varepsilon^{\prime}, R\right) c_{\varepsilon \mu}^{\dagger} c_{\varepsilon^{\prime} \nu}\right] \mathbf{I}_{-}+H . c .,
\end{aligned}
$$

onde $W(\varepsilon, R)$ é dado em (2.17) e $\nu=-\mu$.

Para facilirar a manipulação do Hamiltoniano $H_{\text {probe }}$ definido em (2.23), vamos definir os operadores $\phi_{\mu}(R)$ and $\Phi_{0 \mu}(R)$ como combinações dos $c_{\varepsilon}$ e $\bar{c}_{\varepsilon}$. Então,

$$
\phi_{\mu}(R) \equiv \int_{-D}^{D} d \varepsilon W(\varepsilon, R) c_{\varepsilon \mu}
$$

e

$$
\Phi_{0 \mu}(R) \equiv \int_{-D}^{D} d \varepsilon \sqrt{1-W(\varepsilon, R)} \bar{c}_{\varepsilon \mu}
$$

de modo que a equação (2.23) fica reescrita como

$$
H_{\text {probe }}=-A\left[\Phi_{0 \uparrow}^{\dagger} \Phi_{0 \downarrow}+\phi_{\uparrow}^{\dagger} \phi_{\downarrow}+\left(\Phi_{0 \uparrow}^{\dagger} \phi_{\downarrow}+\phi_{\uparrow}^{\dagger} \Phi_{0 \downarrow}\right)\right] \mathbf{I}_{-}+H . c . .
$$

As relações (2.24) e (2.25) separam os estados $|\psi\rangle$ do Hamiltoniano de Anderson $H_{A}$, dado por

$$
H_{A}=\int_{-D}^{D} \varepsilon c_{\varepsilon \mu}^{\dagger} c_{\varepsilon \mu} d \varepsilon+\sqrt{\frac{\Gamma}{\pi}} \int_{-D}^{D} d \varepsilon\left(c_{\varepsilon}^{\dagger} c_{d}+c_{d}^{\dagger} c_{\varepsilon}\right)+H_{d},
$$

dos estados $|\bar{\psi}\rangle$ de $\bar{H}_{c}$.

Assim, o sistema compreendendo o metal e a impureza não-perturbados e a ponta de prova é representado pelo Hamiltoniano

$$
\tilde{H}=H_{A}+\bar{H}_{c},
$$


cujos autoestados são desacoplados, ou seja,

$$
|\Psi\rangle=|\psi\rangle|\bar{\psi}\rangle
$$

e, portanto, os autovalores podem ser separados em contribuições independentes

$$
E_{\Psi}=E_{\psi}+\bar{E}_{\bar{\psi}}
$$

A relação (2.29) mostra que os $\bar{c}_{\varepsilon}$ são desacoplados da impureza. Desse modo, podemos tratar o operador $\Phi_{0 \mu}(R)$, apresentado na equação (2.25), perturbativamente. Por sua vez, a definição de $\phi_{\mu}(R)$ em (2.24) deve ser projetada na base $\left\{f_{n}\right\}$ do Grupo de Renormalização Numérico - o que mostraremos no capítulo 4.

Com o Hamiltoniano (2.27) definido na base contínua $\left\{c_{\varepsilon}\right\}$, encerramos o tratamento puramente analítico do nosso sistema quântico não-pertubado. A seguir, no capítulo 3, introduzimos os fundamentos do Grupo de Renormalização através dos quais é possível transformar $\tilde{H}$ em uma forma numericamente solúvel. 


\section{O Grupo de Renormalização numérico}

Physicists like to think that all you have to do is say, these are the conditions, now what happens next? Richard P. Feynman

O Grupo de Renormalização Numérico (NRG) $(10,11,30)$ consiste em um método desenvolvido por Wilson (10) na década de 70 na tentativa de tratar sistemas quânticos de muitos corpos com impurezas. Originalmente utilizada no problema Kondo, a técnica de Wilson obteve grande avanço, uma vez que as tentativas analíticas outrora elaboradas para resolver o problema perturbativamente falhavam para baixas energias e, por conseguinte, não contemplavam a escala típica de baixas temperaturas. Em particular, o NRG obteve resultados promissores no cálculo de observáveis físicos - como susceptibilidade magnética e calor específico - em todo o limite de temperaturas de interesse do efeito Kondo (regimes de momento local e acoplamento forte, a altas e baixas temperaturas, respectivamente). Mais tarde, o sucesso do método foi comprovado por Krishna-murthy (11) no modelo de Anderson (12) com uma impureza. Assim, não tardou para que o NRG rendesse a Wilson o Prêmio Nobel (em 1982).

Os conceitos de renormalização, pontos fixos e fluxo iterativos, hoje bastante conhecidos na teoria da Matéria Condensada são ingredientes essenciais do Grupo de Renormalização Numérico. Aplicações recentes do método incluem a diagonalização do Hamiltoniano de diversos modelos de metais com impurezas e ligas diluídas, bem como o cálculo de propriedades de excitação e transporte e correlações espaciais.

Ao longo deste capítulo, apresentaremos os fundamentos do Grupo de Renormalização Numérico e mostraremos como ele será utilizado no nosso problema da medida da nuvem Kondo. 


\subsection{Da Teoria de Renormalização ao Grupo de Renor- malização Numérico}

O Grupo de Renormalização Numérico remete à Teoria de Renormalização devida aos trabalhos de Bethe, Shwinger, Tomonaga, Feynman e Dyson (31-39) para eliminar as divergências em Eletrodinâmica Quântica. O Grupo de Renormalização (RG) foi o passo inicial nas técnicas voltadas ao cálculo do limite estatístico do contínuo em uma teoria local, caracterizado pela ausência de um comprimento característico $\xi$ e de escalas de tempo. Contudo, - RG aplicava-se somente a problemas passíveis de expansão por diagramas de Feynman, ao mesmo tempo que a física no limite do contínuo não era palpável por este método.

A ideia básica do Grupo de Renormalização consiste na existência de um acoplamento local entre várias escalas de comprimento ou de energia (10), de modo que flutuações entre comprimentos (energias) intermediários tendem a ser idênticos a menos de um fator de escala. A medida que consideramos escalas de energia cada vez mais baixas, é produzido um efeito cascata, pois cada pequena mudança em um dado passo afeta todos os demais. A primeira etapa do RG consiste na determinação de um passo particular (isto é, uma escala de energia) da cascata, a partir do qual serão definidos os demais passos (iterações) para a solução do problema de interesse. Nesse processo, a cada iteração é associado um Hamiltoniano $\mathscr{H}_{N}$ representando uma interação efetiva no sistema e acessando as escalas energéticas que ainda não foram resolvidas. Nas próximas iterações, os Hamiltonianos $\mathscr{H}_{N+1}$ são construídos através de uma transformação de renormalização do Hamiltoniano da iteração precedente $\mathscr{H}_{N}$.

A possibilidade do RG de trabalhar a solução de um sistema iterativamente é bastante atrativa ao tratamento numérico. Para tanto, basta elaborar um conjunto de transformações de renormalização dispensando o uso de diagramas de Feynman e capaz de ser implementada em forma de algoritmo em um programa de computador.

À época em que Wilson propôs o NRG, o estudo perturbativo de sistemas quânticos com impurezas era barrado por divergências no limite de baixas temperaturas - o acoplamento spin-spin entre a impureza e a banda de condução apresentava uma divergência no termo quadrático $J^{2} \ln (D / k T)$ no limite $T \rightarrow 0$. Desse modo, a análise de sistemas quânticos com impurezas requeria o desenvolvimento de um método válido simultaneamente para altas energias (graus de liberdade quasi-contínuos do ambiente) e baixíssimas energias de excitação (acoplamento forte da impureza com o restante do sistema). A escolha dos intervalos de energia que caracterizam o sistema na transição entre estes dois regimes deveria permitir o estudo da física contida nos subintervalos energéticos, preservando o limite do contínuo de 
estados.

Nesse contexto, o desafio de resolver o problema Kondo aproveitando o potencial numérico oferecido pelo Grupo de Renormalização foi a motivação para o Grupo de Renormalização Numérico concebido por Kenneth Wilson (40). A solução do problema Kondo apresentada por Wilson (10) foi o primeiro exemplo de implementação de um programa de NRG capaz de incorporar aspectos quantitativos e qualitativos importantes, outrora considerados impossíveis de serem derivados pelas teorias vigentes. O NRG trazia não somente resultados satisfatórios, como também a possibilidade de controlar a precisão dos cálculos, alcançava o valor de até $1 \%$ para sistemas Kondo mais simples (10).

A seguir, apresentaremos a definição formal do Grupo de Renormalização seguido pelas bases do Grupo de Renormalização Numérico aplicado por Wilson ao efeito Kondo.

\subsection{O Grupo de Renormalização}

O Grupo de Renormalização é uma transformação $\tau$ que leva o Hamiltoniano $\mathscr{H}(\mathbf{K})$ especificado pelo conjunto de parâmetros $\mathbf{K}=\left(K_{1}, K_{2}, \ldots\right)$ em um novo Hamiltoniano $\mathscr{H}\left(\mathbf{K}^{\prime}\right)$ com um novo conjunto de parâmetros $\mathbf{K}^{\prime}=\left(K_{1}^{\prime}, K_{2}^{\prime}, \ldots\right)$, isto é,

$$
\tau\{\mathscr{H}(\mathbf{K})\}=\mathscr{H}\left(\mathbf{K}^{\prime}\right)
$$

ou, equivalentemente,

$$
\tau(\mathbf{K})=\mathbf{K}^{\prime}
$$

O Hamiltoniano $\mathscr{H}(\mathbf{K})$ representa uma interação efetiva do sistema para uma dada escala energética. Por sua vez, o Hamiltoniano $\mathscr{H}\left(\mathbf{K}^{\prime}\right)$ corresponde a esta interação em uma escala reduzida. Por conseguinte, deve haver uma razão entre as escalas acessadas por tranformações $\tau$ sucessivas, que pode ser caracterizada por um parâmetro $\Lambda$. Assim, a sequência

$$
\mathbf{K}^{\prime}=\tau_{\Lambda}(\mathbf{K}), \quad \mathbf{K}^{\prime \prime}=\tau_{\Lambda}\left(\mathbf{K}^{\prime}\right), \quad \mathbf{K}^{\prime \prime \prime}=\tau_{\Lambda}\left(\mathbf{K}^{\prime \prime}\right), \quad \text { etc. }
$$

dá origem a uma sequência de pontos que, quando $\Lambda$ é uma variável contínua, torna-se uma trajetória no espaço de parâmetros $\mathbf{K}$. A transformação $\tau_{\Lambda}$, em geral não-linear e nãoinversível, constitui um semi-grupo satisfazendo a propriedade

$$
\tau_{\Lambda^{\prime}}\left\{\tau_{\Lambda}\left(\mathbf{K}^{\prime}\right)\right\}=\tau_{\Lambda^{\prime} \Lambda}(\mathbf{K})
$$


Uma importante característica de uma transformação $\tau_{\Lambda}(\mathbf{K})$ do RG consiste na existência de pontos $\mathbf{K}^{*}$ para os quais vale

$$
\tau_{\Lambda}\left(\mathbf{K}^{*}\right)=\mathbf{K}^{*}
$$

ou seja, a atuação de $\tau_{\Lambda}$ sobre $\mathbf{K}^{*}$ o deixa invariante.

Pontos do tipo $\mathbf{K}^{*}$ satisfazendo (3.5) são denotados pontos fixos e são especiais por trazerem inúmeras vantagens analíticas. A principal delas, diz respeito à possibilidade de linearizar a transformação $\tau$ nas proximidades do ponto fixo $\mathbf{K}^{*}$. Desse modo, dado um ponto $\mathbf{K}=\mathbf{K}^{*}+\delta K$ na vizinhança de $K^{*}$, podemos fazer a seguinte expansão:

$$
\tau_{\Lambda}\{\mathbf{K}\}=\mathbf{K}^{*}+\mathbf{L}_{\Lambda}^{*} \delta \mathbf{K}+O\left(\delta K^{2}\right)
$$

onde $\mathbf{L}_{\Lambda}^{*}$ é uma transformação linear qualquer.

Na seção subsequente mostaremos como os fundamentos do Grupo de Renormalização foram utilizados por Wilson na formulação do Grupo de Renormalização Numérico aplicado ao efeito Kondo.

\subsection{O Grupo de Renormalização Numérico no Pro- blema Kondo}

Como mencionamos, o NRG proposto por Wilson (10) foi a primeira abordagem teórica que obteve sucesso na solução do problema Kondo. Diversos trabalhos precedentes foram devotados ao estudo de metais com impurezas magnéticas no limite de baixas temperaturas. Buscava-se não apenas a confluência entre os resultados teóricos e experimentais, mas também a possibilidade de predizer e comparar o comportamento destes sistemas para $T \rightarrow 0$. Particularmente, o NRG devido a Wilson foi uma continuação dos desenvolvimentos de RG derivados em trabalhos precedentes (41-44), cujos principais resultados mostravam-se válidos somente para altas temperaturas.

A essência do NRG de Wilson no problema Kondo consiste em transformar o Hamiltoniano de Kondo de tal modo que ele seja solúvel numericamente. Na prática, isso equivale a reescrever o Hamiltoniano de Kondo em uma base de estados apropriada e desenvolvê-lo iterativamente a partir de uma transformação de renormalização, como em (3.1). Para tanto, a banda de condução é associada a uma base infinita de estados discretos, denominada base de Kondo, distribuídos ao redor da impureza de acordo com o parâmetro $\Lambda$. Pictoricamente, podemos 
ilustrar estes estados por meio cascas concêntricas ao redor da impureza, como mostra a figura 3.1.

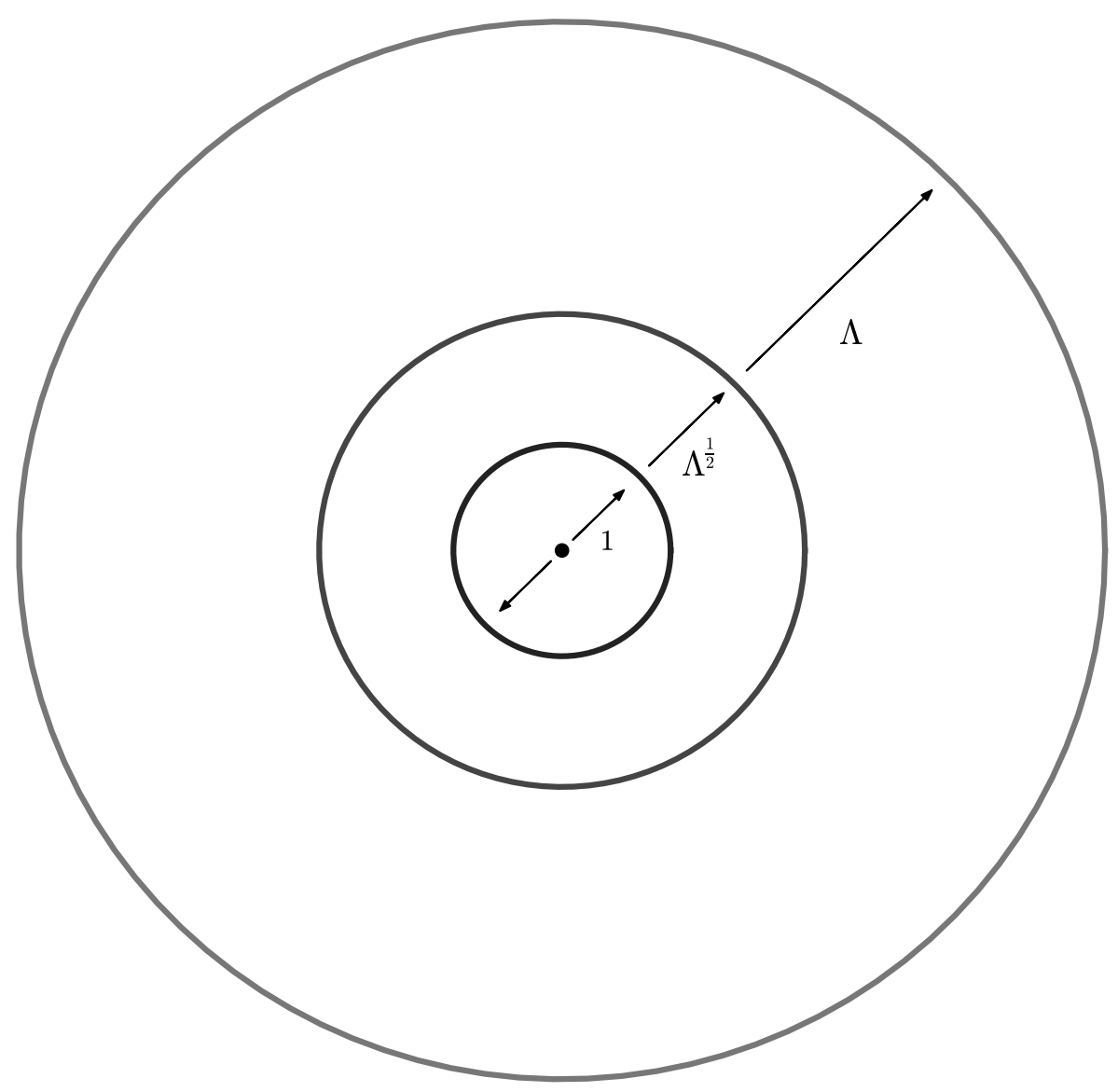

Figura 3.1 - Cascas concêntricas em torno da impureza representando os estados na base Kondo. Fonte: WILSON, K. G. (10).

Uma vez definida a base discreta referente à banda de condução, a solução iterativa do Hamiltoniano pode ser obtida através da adição sucessiva de estados da base de Kondo ao estado da impureza: na iteração inicial, resolvemos um sistema que equivale à impureza acoplada com o primeiro estado da banda de condução; na próxima iteração, adicionamos os segundos estados da banda de condução e resolvemos o Hamiltoniano para a combinação desses estados com os primeiros e com a impureza; nas próximas iterações, adicionamos o terceiro estado, seguido pelo quarto estado, e assim sucessivamente.

Obviamente, o tratamento numérico do problema exige um limite para o numéro máximo de iterações a serem processadas. Ao mesmo tempo, o aumento do número de estados e suas combinações ao longo das iterações torna a computação cara em tempo e memória. Ambas limitações podem ser solucionadas através de truncamentos: o número de iterações 
fica condicionado ao corte infravermelho associado à escala térmica de interesse, ao passo que o número de estados em cada iteração fica limitado pela energia ultravioleta.

Em suma, a técnica NRG para o problema Kondo pode ser resumida nas etapas a seguir:

1. Discretização da banda de condução em escala logarítmica, definida por um parâmetro adimensional $\Lambda$.

2. Mudança de base para obtenção de uma cadeia infinita representando a banda discretizada. Normalmente, esta etapa é guiada por uma Transformação de Lanczos (45) que gera, por recorrência, uma base $\left\{f_{n}\right\}$ com $f_{0}$ sendo o operador que representa a semente da transformação.

3. Truncamento da cadeia infinita que define o Hamiltoniano, limitando o número máximo de iterações a um valor inteiro $N$ tal que os coeficientes da escala de energia da banda de condução $\mathscr{E}(\Lambda, N)$ são bem menores do que $k_{B} T / D$.

4. Diagonalização iterativa do Hamiltoniano do sistema. Uma vez diagonalizado o Hamiltoniano $H_{N-1}$, descartam-se ses autoestados com energias muito superiores a $k_{B} T$, projeta-se o Hamiltoniano $H_{N}$ na base de autovetores remanescentes e diagonaliza-se a matriz resultante. A cada iteração, resulta o espectro e os autovetores necessários para se calcular observáveis físicos.

Nas próximas seções, exploraremos cada uma dessas etapas, detalhando sua motivação e justificando as aproximações.

\subsection{Discretização da banda de condução}

O passo inicial para a solução do problema Kondo via NRG consiste em representar o Hamiltoniano de Kondo em uma base quântica capaz de reproduzir numericamente o espectro contínuo associado à banda de condução e que, ao mesmo tempo, permita acessar todas as escalas energéticas de interesse. Em outras palavras, devemos discretizar a banda de condução de modo que cada estado da base seja associado a um intervalo energético com escala definida.

Em princípio, poderíamos ser tentados a dividir a banda de condução em uma escala linear, porém esta escolha nos levaria a introduzir energias características que não existem no sistema, além de limitar o cálculo para temperaturas $T$, tal que $k_{B} T \gg \Delta$, onde $\Delta$ é o espaçamento entre os intervalos energéticos definidos pela discretização linear. 
A escolha da discretização logarítmica é conveniente neste tipo de problema, pois no cálculo de muitas propriedades físicas do sistema aparecem integrais da energia da forma

$$
\int_{E_{0}}^{\infty} \frac{d \varepsilon}{\varepsilon},
$$

cujo resultado $\ln (\varepsilon)$, diverge no limite superior.

Com a discretização logarítmica, a banda de condução pode ser representada por uma cadeia infinita de estados, ao passo que a interação entre a impureza e os elétrons do metal é feita pelo acoplamento com um único estado. Mais detalhes na seção 3.5, equação (3.23). Em termos práticos, a divisão logarítmica permite alcançar escalas de energias tão pequenas quanto se queira, separando altas de baixas energias. Em termos computacionais, sua principal vantagem diz respeito ao número de intervalos necessários para se atingir valores típicos de energia do sistema, que na escolha da escala linear pode chegar a $4^{10^{6}}$ !

A discretização logarítmica é feita dividindo o intervalo energético da banda de condução $[-D, D]$ em subintervalos modelados por um parâmetro adimensional $\Lambda>1$, que é a razão entre duas energias discretas sucessivas. Dada uma energia $\varepsilon_{k}$ no Hamiltoniano da banda de condução, sempre podemos encontrar $\varepsilon_{k}^{\prime}=\varepsilon_{k} / \Lambda$ tal que a divisão resulta em intervalos energéticos definidos por

$$
\mathscr{I}_{m \pm}=\left[D \Lambda^{-m-1}, D \Lambda^{-m}\right] \quad(m=0,1,2, \ldots)
$$

de tamanho $d_{m}=D \Lambda^{-m}\left(1-\Lambda^{-1}\right)$ entre $[-D, D]$. Note que $\mathscr{I}_{m \pm} \rightarrow \mathscr{I}_{m+1 \pm}$ é invariante sobre a transformação de escala $\varepsilon_{k} \rightarrow \varepsilon_{k} / \Lambda$. V

A equação (3.8) determina uma sequência de intervalos $\mathscr{I}_{m}$ tais que a razão entre intervalos sucessivos é $\Lambda$. Esse processo dá origem à discretização logarítmica da banda de condução ilustrado na figura 3.2 .

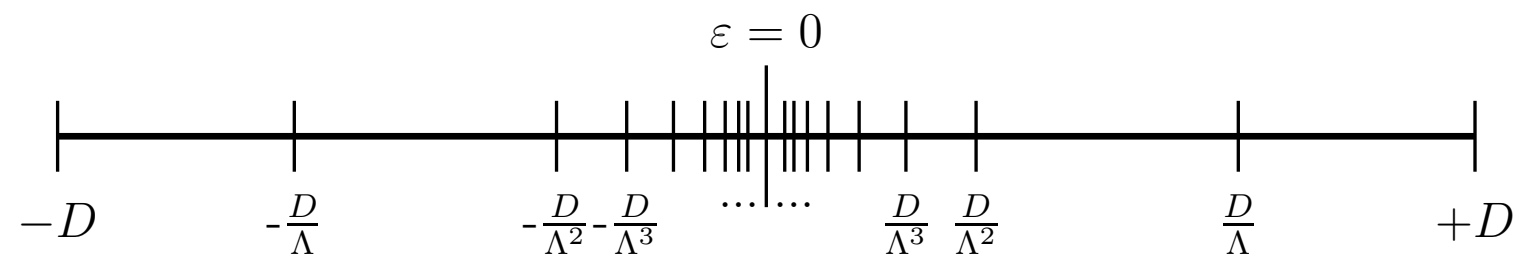

Figura 3.2 - A banda de condução é discretizada em escala logarítimica, definida por um parâmetro adimensional $\Lambda$. Fonte: elaborada pela autora. 
Uma vez definidos os intervalos energéticos discretizando a banda de condução, prosseguimos com o método NRG construindo a base de operadores fermiônicos $\left\{a_{m}, b_{m}\right\}$ associados aos intervalos $\mathscr{I}_{m \pm}$ e apresentando o conjunto de transformações para a base de Kondo $\left\{f_{n}\right\}$ na qual a o Hamiltoniano $H_{A}$ é solúvel numericamente.

\subsection{Mudança de base e Transformação de Lanczos}

Dentro de cada um dos intervalos $\mathscr{I}_{m \pm}$ apresentados em (3.8), definimos o conjunto de operadores fermiônicos $a_{m \mu}\left(b_{m \mu}\right)$ e $\bar{a}_{m \mu}\left(\bar{b}_{m \mu}^{\dagger}\right)$ como combinações dos $c_{\varepsilon \mu}$. Não é necessário discretizar os $\bar{c}_{\varepsilon}$ : eles serão tratados analiticamente* no cálculo da taxa $1 / T_{1}$. Segue que

$$
\begin{aligned}
& a_{m \mu}=\frac{\Lambda^{m / 2}}{\sqrt{D\left(1-\Lambda^{-1}\right)}} \int_{\mathcal{I}_{m+}} c_{\varepsilon \mu} d \varepsilon, \\
& b_{m \mu}=\frac{\Lambda^{m / 2}}{\sqrt{D\left(1-\Lambda^{-1}\right)}} \int_{\mathcal{I}_{m-}} c_{\varepsilon \mu} d \varepsilon,
\end{aligned}
$$

Na base $\left\{a_{m}, b_{m}\right\}$, o Hamiltoniano da banda de condução em (2.3) fica expresso na forma

$$
H_{\text {cond }}=\sum_{m \pm} \mathscr{E}_{m \pm}(\Lambda)\left(a_{m \mu}^{\dagger} a_{m \mu}-b_{m \mu}^{\dagger} b_{m \mu}\right)
$$

onde as energias $\mathscr{E}_{m \pm}$ são calculadas como

$$
\mathscr{E}_{m \pm}(\Lambda)=\frac{\int_{\mathscr{I}_{m \pm}} d \varepsilon}{\int_{\mathscr{I}_{m \pm}} \frac{d \varepsilon}{\varepsilon}}= \pm \frac{D\left(1-\Lambda^{-1}\right)}{\log \Lambda} \Lambda^{-m}
$$

onde utilizamos a expressão de Vivaldo $(46,47)$ derivada na formulação generalizada do NRG.

Cada energia $\mathscr{E}_{m \pm}(\Lambda)$ corresponde à média da energia cinética dos elétrons livres dentro do intervalo $\mathscr{I}_{m \pm}$.

Agora, a partir da base $\left\{a_{m \mu}, b_{m \mu}\right\}$, vamos construir a base ortogonal $\left\{f_{n}\right\}$ na qual o $\mathrm{Ha}$ miltoniano Kondo será resolvido via NRG. Para tanto, definimos os operadores $f_{n}$ como sendo combinações dos $a_{m}$ e $b_{m}$ com os respectivos coeficientes $u_{n m}$ e $v_{n m}$ sendo transformações ortogonais. Matematicamente, escrevemos

$$
f_{n \mu}=\sum_{m}\left(u_{n m} a_{m \mu}+v_{n m} b_{m \mu}\right)
$$

*Mais detalhes no Capítulo 4. 
valendo as transformações inversas:

$$
\begin{aligned}
& a_{m \mu}=\sum_{n} u_{n m} f_{n \mu}, \\
& b_{m \mu}=\sum_{n} v_{n m} f_{n \mu},
\end{aligned}
$$

A base $\left\{f_{n}\right\}$ deve satisfazer as seguintes relações exigidas pela ortogonalidade:

$$
\begin{gathered}
\sum_{n} u_{n m} u_{n m^{\prime}}=\delta_{m, m^{\prime}}, \\
\sum_{n} u_{n m} v_{n m^{\prime}}=0, \\
\sum_{n} v_{n m} v_{n m^{\prime}}=\delta_{m, m^{\prime}}, \\
\sum_{m} u_{n m} u_{n^{\prime} m}+v_{n m} v_{n^{\prime} m}+=\delta_{n, n^{\prime}}, \\
v_{n m}=(-1)^{n} u_{n m} .
\end{gathered}
$$

Vamos também definir o operador $f_{0}$ :

$$
f_{0 \mu}=\frac{1}{\sqrt{2}} \sum_{m} \alpha_{m}\left(a_{m \mu}+b_{m \mu}\right)
$$

onde

$$
\alpha_{m}=\left(\frac{1-\Lambda^{-1}}{D}\right)^{1 / 2} \Lambda^{-m / 2}
$$

Então, usando a eq. (3.21), o acoplamento entre a impureza e a banda de condução, descrito pelo Hamiltoniano (2.6), pode ser reescrito como

$$
H_{\text {int }}=\sqrt{2} V\left(c_{d}^{\dagger} f_{0}+f_{0}^{\dagger} c_{d}\right) .
$$

Portanto, no Hamiltoniano de interação (3.23), a impureza se acopla com um único estado da base $f_{0}$, resultando em um termo que não é afetado pela discretização.

Usando a transformação de Lanczos (45), admitimos que cada operador $f_{n \mu}$ se acopla 
com $f_{n \pm 1 \mu}$. Então, $H_{\text {cond }}$ em (3.11) fica escrito na base $\left\{f_{n}\right\}$ como

$$
H_{\text {cond }}=\sum_{n=0}^{\infty} t_{n}\left(f_{n}^{\dagger} f_{n+1}+f_{n+1}^{\dagger} f_{n}\right)
$$

onde os coeficientes energéticos $t_{n}$ são dados por (11)

$$
t_{n}=D \Lambda^{-n / 2} \frac{1-\Lambda^{-(n+1)}}{\sqrt{1-\Lambda^{-(2 n+3)}} \sqrt{1-\Lambda^{-(2 n+1)}}} \frac{1+\Lambda^{-1}}{\log \Lambda},
$$

Para $n$ suficientemente grande, os coeficientes $t_{n}$ da última equação (3.25) comportam-se assintoticamente como

$$
t_{n} \approx \mathcal{D}_{n}=D \frac{1-\Lambda^{-1}}{\log \Lambda} \Lambda^{-n / 2}
$$

com erro $O\left(\Lambda^{-n}\right)$.

Expandindo a soma do Hamiltoniano (3.24), é possível determinar os coeficientes $u_{n, m} \mathrm{e}$ $v_{n, m}$ da transformação (3.13). Em particular, para $n=0$, comparando as relações (3.21) e (3.24), estes coeficientes são definidos pela relação

$$
u_{0 m}=v_{0 m}=D\left(\frac{1-\Lambda^{-1}}{\log \Lambda}\right)^{1 / 2} \Lambda^{-m / 2}
$$

Para $n=1$, substituímos (3.27) para encontrar os $u_{1 m}$ and $v_{1 m}$, de modo que

$$
u_{1 m}=-v_{1 m}=D\left(\frac{1-\Lambda^{-3}}{\log \Lambda}\right)^{1 / 2} \Lambda^{-3 m / 2}
$$

As equações (3.24), (3.27) e (3.28) indicam uma relação de recorrência envolvendo os $u_{1 m}$ e os $u_{0 m}$. Em geral, podemos expandir a soma em $H_{\text {cond }}$ até $N+1$ e compará-la com a soma expandida até $N$. Coletando os termos $u_{n m}, u_{n+1 m}$ e $u_{n-1 m}$, é possível generalizar a recorrência para todos os elementos da cadeia, ou seja,

$$
\begin{gathered}
t_{n-1} u_{n-1, m}+t_{n} u_{n+1, m}=\mathcal{D}_{2 m} u_{n m} \\
t_{n-1} v_{n-1, m}+t_{n} v_{n+1, m}=-\mathcal{D}_{2 m} v_{n m}
\end{gathered}
$$

O Hamiltoniano na eq. (3.24) é conhecido como Hamiltoniano de "hopping", uma vez que o acoplamento de cada operador $f_{n}$ com $f_{n+1}$ é análogo a uma troca (ou "pulo") de elétrons entre sítios vizinhos, como ilustra a figura 3.3. Esse Hamiltoniano não contém termos diagonais $f_{n}^{\dagger} f_{n}$, pois a energia média de um estado criado pelo operador $f_{n}$ será a energia de Fermi, que já foi descontada. 


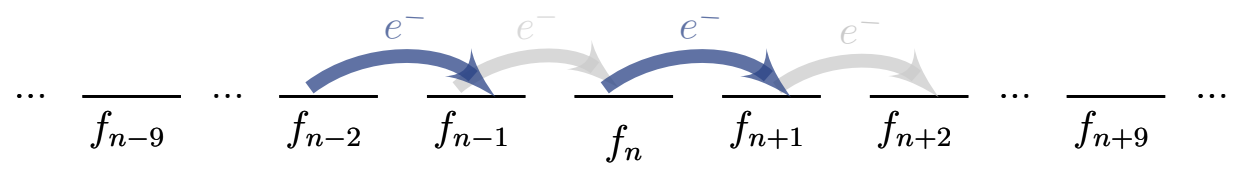

Figura 3.3 - Representação do hopping de elétrons entre os sítios $f_{n}$ da banda de condução. Cada elétron em um sítio $f_{n}$ pode ser transferido para um sítio $f_{n+1}$ e vice-versa. Fonte: elaborada pela autora.

Finalmente, juntamos as equações (2.5), (3.23) e (3.24) para escrever o Hamiltoniano (2.1) na base discreta $\left\{f_{n}, c_{d}\right\}$, isto é,

$$
H=\sum_{n=0}^{\infty} t_{n}\left(f_{n}^{\dagger} f_{n+1}+H . c .\right)+\sqrt{2} V\left(c_{d}^{\dagger} f_{0}+H . c .\right)+H_{d} .
$$

Com a equação (3.31), conseguimos obter uma expressão para o Hamiltoniano que resolve a questão da discretização. Contudo, o Hamiltoniano $H_{A}$ na base $\left\{f_{n}, c_{d}\right\}$ ainda não é numericamente tratável, pois embute somas infinitas nos termos em $f_{n}$ e $f_{0}$ - veja as eqs.(3.24) e (3.21). Para superar esta dificuldade devemos truncar a série infinita que aparece na eq. (3.24), preservando o termo de acoplamento.

Uma vez que os coeficientes $t_{n}$ em (3.24) e $\alpha_{m}$ em (3.21) decaem exponencialmente com $n$, poderíamos ser tentados a truncar ambas as somas em (3.24) e (3.21). Entretanto, isto afetaria o operador $f_{0}$ e tornaria a precisão do cálculo dependente da magnitude de $V$ multiplicando $f_{0}$. Truncando somente a soma da equação (3.24), o acomplamento é preservado.

Dada uma energia térmica $k_{B} T$, onde $k_{B}$ é a constante de Boltzman, e um parâmetro adimensional $\gamma \ll 1$, podemos limitar a soma infinita em (3.24). O Hamiltoniano $H_{\text {cond }}$ truncado torna-se

$$
H_{\text {cond }}^{\text {trunc }}=\sum_{n=0}^{N-1} t_{n}\left(f_{n}^{\dagger} f_{n+1}+f_{n+1}^{\dagger} f_{n}\right)
$$

onde $N$ é o mínimo inteiro satisfazendo

$$
t_{N}<\gamma k_{B} T
$$

Tendo determinado o número máximo de iterações com a condição (3.33), vamos obter a transformação de renormalização para a solução do problema Kondo.

É conveniente escalar o Hamiltoniano de tal modo que o menor autovalor proveniente da diagonalização seja da ordem da unidade, isto é,

$$
H_{N}=\frac{1}{\mathcal{D}_{N}}\left(\sum_{n=0}^{N-1} t_{n}\left(f_{n}^{\dagger} f_{n+1}+H . c .\right)+\sqrt{2} V\left(c_{d}^{\dagger} f_{0}+H . c .\right)+H_{d}\right),
$$


onde $\mathcal{D}_{N}$ é dado em (3.26).

A equação (3.34) define uma transformação de Grupo de Renormalização, ou seja, ela constitui uma transformação que adiciona progressivamente escalas de energia cada vez menores ao Hamiltoniano, tal que as menores energias são da ordem da unidade. Se denotarmos esta transformação por $\tau$, segue que

$$
\tau\left[H_{N-1}\right] \equiv H_{N}=\sqrt{\Lambda} H_{N-1}+\frac{t_{N-1}}{\mathcal{D}_{N}}\left(f_{N-1}^{\dagger} f_{N}+H . c .\right) .
$$

Por meio da transformação $\tau$ em (3.35), podemos construir o ciclo iterativo para a diagonalização do Hamiltoniano de Kondo.

\subsection{Diagonalização iterativa}

Podemos interpretar fisicamente o Hamiltoniano $H_{N}$ como um conjunto de $N+1$ sítios, tal que cada sítio pode conter nenhum, um ou dois elétrons. Portanto, cada iteração possui $2^{2 N+4}$ estados de muitos corpos.

Ao adicionar sítios e elétrons, modificamos a carga e o spin do sistema. Uma vez que a projeção (3.34) do Hamiltoniano na base $\left\{f_{n}, c_{d}\right\}$ conserva carga e spin, podemos escrever os operadores $Q$ e $S$ associados nessa base como

$$
Q=\left(c_{d}^{\dagger} c_{d}-1 / 2\right)+\sum_{n=0}^{N}\left(f_{n}^{\dagger} f_{n}-1 / 2\right),
$$

e

$$
\vec{S}=\frac{1}{2}\left(c_{d \mu}^{\dagger} \vec{\sigma}_{\mu \nu} c_{d \nu}+\sum_{n=0}^{N} f_{n \mu}^{\dagger} \vec{\sigma}^{\mu \nu} f_{n \nu}\right),
$$

onde $\sigma_{i}(i=x, y, z)$ são as matrizes de Pauli.

Uma observação importante com respeito à carga medida pelo operador $Q$ é que os valores que ela assume estão no intervalo $[-(N+2),+(N+2)]$. Assim, atribuímos a carga mínima ao estado com 0 elétrons e a carga máxima ao estado com o máximo número de elétrons posível para uma dada iteração (que é $2 N+4$ na $\mathrm{N}$-ésima iteração). Por exemplo, na iteração $N=-1$, os possíveis valores de carga dos estados da impureza são $q \in\{-1,0,1\}$, na iteração $N=0$ são $q \in\{-2,-1,0,1,2\}$, na iteração $N=1$ são $q \in\{-3,-2,-1,0,1,2,3\}$, etc.

Dadas as leis de conservação do sistema, é natural representarmos o Hamiltoniano na base de autoestados simultâneos dos operadores $Q, S$ e $S_{z}$. O uso da base $\left\{\left|Q, S, S_{z}\right\rangle\right\}$ é 
conveniente, pois a projeção do Hamiltoniano nos estados da base é uma matriz diagonal por blocos. Cada bloco contém $p_{\max }<N$ estados $\left|Q, S, S_{z}, p\right\rangle\left(r=1, \ldots, p_{\max }\right)$ de mesma carga, spin e componente $z$ do spin, de modo que seus autovalores e autovetores podem ser facilmente computados através das rotinas tradicionais de diagonalização.

Para ilustrar esta ideia, imagine que estamos na iteração $N=-1$. Nesta situação, temos um único sítio $c_{d}$ que representa a impureza e os 4 estados que ela pode ter, como mostra a figura 3.4.

Obter a iteração $N=0$ a partir da iteração $N=-1$ significa acrescentar o sítio $f_{0}$ ao sítio $c_{d}$ e gerar todas as configurações eletrônicas possíveis para estes dois sítios que obeceçam ao Princípio de Exclusão de Pauli e às regras de soma de spins. Veja a Figura 3.5.

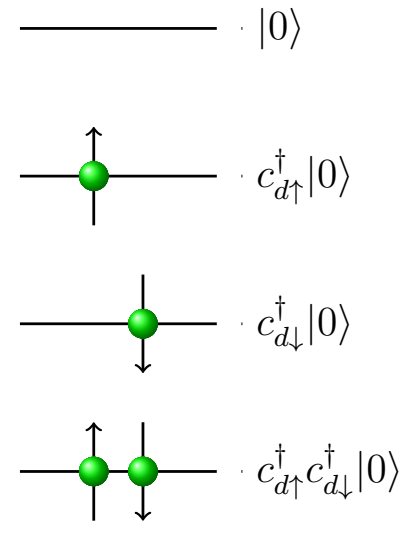

Figura 3.4 - Representação dos 4 estados de muitos corpos na iteração $N=-1$. Há apenas o sítio da impureza com quatro possíveis configurações eletrônicas: cada estado $|0\rangle, c_{d \uparrow}^{\dagger}|0\rangle$, $c_{d_{\downarrow}}^{\dagger}|0\rangle$ and $c_{d \uparrow}^{\dagger} c_{d \downarrow}^{\dagger}|0\rangle$ pode ter, respectivamente, 0 elétrons, 1 elétron com spin up, um elétron com spin down, ou dois elétrons com spins opostos. Fonte: elaborada pela autora.

Outra simplificação que pode ser feita é aproveitar a simetria em $S_{z}$ para economizar no cálculo de energias degeneradas, já que estados de mesma carga $Q$ e mesmo spin $S$, diferindo apenas pelo sinal de $S_{z}$, são degenerados. Assim, estados com cargas e spins iguais formam subespaços ou setores $(Q, S)$, onde os cálculos são amigáveis. 


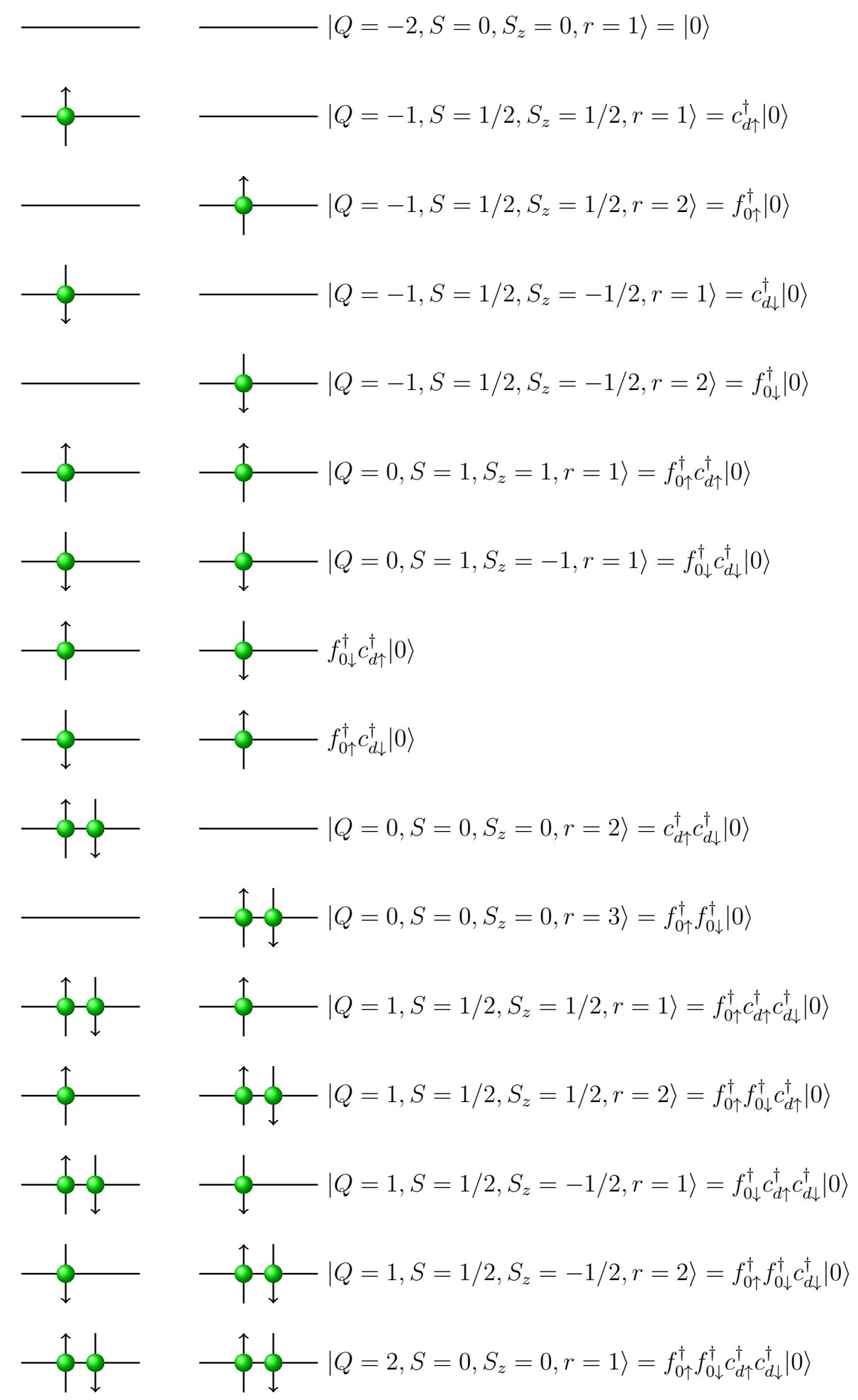

Figura 3.5 - Ilustração dos 16 estados de muitos corpos na iteração $N=0$. Nesta iteração, participam os sítios $c_{d}$ e $f_{0}$, e o estado de maior carga possui dois elétrons em cada um destes sítio. Os estados que não foram identificados como $\left|Q, S, S_{z}, r\right\rangle$ correspondem às combinações singleto e tripleto de $c_{d \uparrow}^{\dagger} f_{0 \downarrow}^{\dagger}|0\rangle$ e $c_{d_{\downarrow}}^{\dagger} f_{0 \uparrow}^{\dagger}|0\rangle$. Fonte: elaborada pela autora. 
Para obtermos o conjunto de autovalores e autovetores do Hamiltoniano $H_{N}$ na iteração $N$, projetamos este operador na base de estados de cada setor $(Q, S)$ e diagonalizamos as matrizes que representam os blocos de $H_{N}$. Pelo menos na primeira iteração (que pode ser $N=-1$ ou $N=0$ ) este cálculo é feito analiticamente. Nas próximas iterações $N+1$, o Hamiltoniano $H_{N+1}$ é construído a partir da a transformação de renormalização (3.35) que relaciona os elementos de $H_{N+1}$ aos autovalores de $H_{N}$ e aos elementos de matriz $\left\langle Q, S, S_{z}, r\left|f_{N}^{\dagger}\right| Q^{\prime}, S^{\prime}, S_{z}^{\prime}, r^{\prime}\right\rangle$. A base de estados na nova iteração $\left\{\left|q, s, s_{z}, r\right\rangle_{N+1}\right\}$ também pode ser construída a partir da base da iteração anterior $\left\{\left|Q, S, S_{z}, p\right\rangle_{N}\right\}$, lembrando que a adição de sítios e elétrons corresponde à mudança de carga e spin com as regras de seleção

$$
\begin{aligned}
& Q^{\prime}=Q+1 \\
& S^{\prime}=S \pm \frac{1}{2}
\end{aligned}
$$

A relação de herança entre os setores pais e filhos faz com que o número de estados aumente rapidamente entre iterações sucessivas. Isso é muito custoso computacionalmente, pois requer memória para armazenar muitos autovalores, autovetores e elementos de matriz, enquanto aumenta o tempo de processamento de todos estes dados.

É conveniente truncarmos o espectro de $H_{N}$, mantendo apenas autovalores $\lambda_{i}<E_{U V}$, onde $E_{U V}$ é a energia de corte Ultravioleta $U V$ adimensional. Na prática, após a diagonalização de $H_{N}$ na base de estados de um setor $(Q, S)$, mantemos apenas os $R(Q, S)<L(Q, S)$ autovalores e seus respectivos autoestados $\left|Q, S, S_{z}, r_{i}\right\rangle$. Os últimos entram no cálculo dos elementos de matriz e na construção da base primitiva da iteração $N+1$.

Em suma, o ciclo iterativo da solução de $H_{N}$ compreende as etapas: construção da base primitiva $\left\{\left|q, s, s_{z}, p\right\rangle_{N}\right\}$ na qual o Hamiltoniano $H_{N}$ é projetado, diagonalização de $H_{N}$, truncamento dos estados com energias $\lambda_{i}>E_{U V}$, cálculo dos elementos de matriz reduzidos $\left\langle q, s, s_{z}, r\left\|f_{N}^{\dagger}\right\| q^{\prime}, s^{\prime}, s_{z}^{\prime}, r^{\prime}\right\rangle$, também conhecidos como invariantes. Ilustramos o fluxo computacional na Figura 3.6.

A seguir, apresentaremos cada uma das etapas envolvidas no ciclo.

\subsubsection{Iteração $N=-1$}

O princípio de nossa solução consiste na diagonalização do Hamiltoniano (3.34) para $N=-1$ na base de 4 estados de muitos corpos ilustrada na figura (3.4). Na representação 


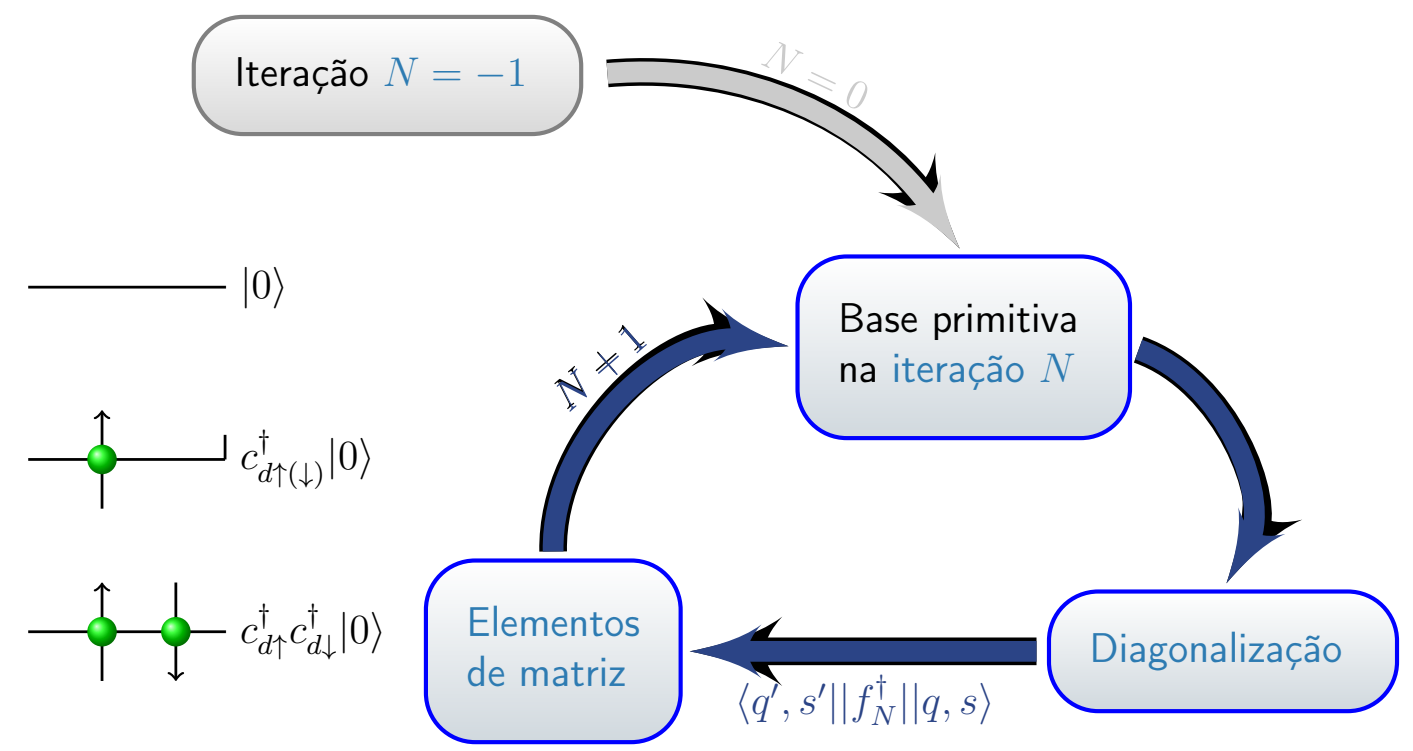

Figura 3.6 - Ciclo iterativo do NRG na solução do problema Kondo. A partir da solução analítica na iteração $N=-1$, construímos a base primitiva da iteração $N=0$ que inicia o ciclo iterativo. No restante das iterações $N$, construímos a base primitiva na qual o Hamiltoniano $H_{N}$ é diagonalizado. Os autovalores e autovetores de $H_{N}$ são armazenados para o cálculo de propriedades físicas e para a construção da iteração $N+1$. Fonte: elaborada pela autora.

$\left|Q, S, S_{z}, r\right\rangle$ esta é dada por

$$
\begin{gathered}
\left|Q=-1, S=0, S_{z}=0,1\right\rangle=|0\rangle \\
\left|Q=0, S=1 / 2, S_{z}=1 / 2,1\right\rangle=c_{d \uparrow}^{\dagger}|0\rangle \\
\left|Q=0, S=1 / 2, S_{z}=-1 / 2,1\right\rangle=c_{d \downarrow}^{\dagger}|0\rangle \\
\left|Q=1, S=0, S_{z}=0,1\right\rangle=c_{d \uparrow}^{\dagger} c_{d \downarrow}^{\dagger}|0\rangle .
\end{gathered}
$$

Nesta iteração, o Hamiltoniano do sistema toma a forma

$$
H_{-1}=\tilde{V}\left(c_{d}^{\dagger} f_{0}+H . c .\right)+\tilde{\varepsilon_{d}} c_{d}^{\dagger} c_{d}+\tilde{U} n_{d \uparrow} n_{d \downarrow}
$$

$\operatorname{com} \tilde{V}=\frac{\sqrt{2 \Gamma / \pi}}{\mathcal{D}_{-1}}, \tilde{\varepsilon_{d}}=\frac{\varepsilon_{d}}{\mathcal{D}_{-1}}$ e $\tilde{U}=\frac{U}{\mathcal{D}_{-1}}$

Os setores $Q, S$ que representam os subespaços de estados onde $H_{-1}$ é diagonalizado são $Q=-1, S=0, Q=0, S=1 / 2$ e $Q=1, S=0$. Desse modo, determinamos as matrizes Hamiltoniano em cada setor $Q, S$ e encontramos seus autovalores e autovetores.

- $(Q=-1, S=0)$

Neste setor a base é composta por um único estado $\left|Q=-1, S=0, S_{z}=0,1\right\rangle=|0\rangle$, que é o próprio autovetor de $H_{-1}$ no subespaço. O Hamiltoniano $H_{-1}$ neste subespaço 
tem dimensão 1 , pois

$$
\left(\left\langle 0\left|H_{-1}\right| 0\right\rangle\right)=(0)
$$

com autovalor $\lambda_{1}=0$ e autovetor $|0\rangle$.

- $(Q=0, S=1 / 2)$

Neste setor a base é composta por dois estados degenerados: $\left|Q=0, S=1 / 2, S_{z}=1 / 2,1\right\rangle=$ $c_{d \uparrow}^{\dagger}|0\rangle=|1\rangle$ e $\left|Q=0, S=1 / 2, S_{z}=-1 / 2,1\right\rangle=c_{d \downarrow}^{\dagger}|0\rangle=|2\rangle$. O Hamiltoniano $H_{-1}$ pode ser projetado em qualquer um dos dois estados $|1\rangle$ ou $|2\rangle$, isto é,

$$
\left(\left\langle 1\left|H_{-1}\right| 1\right\rangle\right)=\left(\left\langle 2\left|H_{-1}\right| 2\right\rangle\right)=\left(\tilde{\varepsilon_{d}}\right),
$$

com autovalor $\lambda_{2}=\tilde{\varepsilon_{d}}$ e autovetor $|1\rangle$ ou $|2\rangle$.

Dada a simetria em $S_{z}$, podemos escolher qualquer um dos dois autovetores.

- $(Q=1, S=0)$

Neste setor a base é composta por um único estado $\left|Q=1, S=0, S_{z}=0,1\right\rangle=c_{d \uparrow}^{\dagger} c_{d \downarrow}^{\dagger}|0\rangle=$ $|3\rangle$, que é o próprio autovetor de $H_{-1}$ no subespaço. que é o autovetor de $H_{1}$ Assim como o setor $(Q=-1, S=0)$ O Hamiltoniano $H_{-1}$ neste subespaço tem dimensão 1 . Veja:

$$
\left(\left\langle 3\left|H_{-1}\right| 3\right\rangle\right)=\left(2 \tilde{\varepsilon_{d}}+\tilde{U}\right)
$$

O autovalor e o autovetor associado à matriz $H_{-1}$ em (3.47) são $2 \tilde{\varepsilon_{d}}+\tilde{U}$ e $|3\rangle$, respectivamente.

Obtido o espectro $\vec{\lambda}=\left(\lambda_{i}\right)(i=0,1,2)$ de $H_{-1}$, determinamos a energia do estado fundamental $E_{g}=\min \left(\lambda_{i}\right)$. Então, descontamos $E_{g}$ de cada autovalor $\lambda_{i}$ de modo que todas as energias sejam medidas a partir do estado fundamental.

Antes de prosseguirmos para a iteração $N=0$, devemos determinar os elementos de matriz $\left\langle Q^{\prime}, S^{\prime}, S_{z}^{\prime}\left|c_{d \mu}^{\dagger}\right| Q, S, S_{z}\right\rangle(\mu=\uparrow, \downarrow)$ do operador $c_{d \mu}^{\dagger}$ entre os pares de estados em (3.40). Uma vez que o operador $c_{d \mu}^{\dagger}$ atua em um estado $\left|Q, S, S_{z}\right\rangle$ modificando sua carga e seu spin, calculamos apenas os elementos de matriz entre estados satisfazendo as regras de seleção (3.38), (3.39) e $S_{z}^{\prime}=S_{z}+\mu$.

Uma simplificação conveniente consiste em computar os elementos de matriz reduzidos $\left\langle Q^{\prime}, S^{\prime}\left\|c_{d \mu}^{\dagger}\right\| Q^{\prime}, S^{\prime}\right\rangle$, também conhecidos como invariantes. Independentes de $S_{z}$, os invariantes são relacionados com os elementos de matriz através do Teorema de Wigner-Eckart 
(48)

$$
\left\langle Q+1, S^{\prime}, s_{z}+\mu\left|f_{N \mu}^{\dagger}\right| Q, S, S_{z}\right\rangle=\left\langle Q+1, S^{\prime}\left\|f_{N \mu}^{\dagger}\right\| Q, S\right\rangle\left(\begin{array}{c|cc}
S^{\prime} & \frac{1}{2} & S \\
S_{z}+\mu & \mu & S_{z}
\end{array}\right),
$$

onde o fator em parênteses é o coeficiente de Clebsch-Gordan para a adição de spin $\vec{S}=\overrightarrow{S^{\prime}}+\vec{s}$, com $\vec{s}$ sendo uma variável de spin $1 / 2$.

Na nossa notação, definimos

$$
\left\langle j_{1}, m_{1} ; j_{2}, m_{2} \mid j, m\right\rangle=\left(\begin{array}{c|cc}
j & j_{2} & j_{1} \\
m & m_{2} & m_{1}
\end{array}\right) .
$$

Em termos computacionais, o teorema de Wigner-Eckart é proveitoso à economia de memória, já que qualquer cálculo que dependa explicitamente de $S_{z}$ pode ser obtido através dos invariantes multiplicados pelos coeficientes de Clebsch-Gordan apropriados.

Na iteração $N=-1$, os elementos de matriz permitidos pelas regras de seleção (3.38) e (3.39) são:

$$
\begin{aligned}
& \left\langle Q=1, S=0, S_{z}+\mu, 1\left|c_{d \mu}^{\dagger}\right| Q=0, S=1 / 2, S_{z}=1 / 2,1\right\rangle \\
& =\left\langle Q=1, S=0\left\|c_{d}^{\dagger}\right\| Q=0, S=1 / 2\right\rangle\left(\begin{array}{c|cc}
0 & \frac{1}{2} & \frac{1}{2} \\
\frac{1}{2}+\mu & \mu & \frac{1}{2}
\end{array}\right)=-\sqrt{2},
\end{aligned}
$$

e

$$
\begin{aligned}
& \left\langle Q=1, S=1 / 2, S_{z}+\mu, 1\left|c_{d \mu}^{\dagger}\right| Q=-1, S=0, S_{z}=0,1\right\rangle \\
& =\left\langle Q=1, S=1 / 2\left\|c_{d \mu}^{\dagger}\right\| Q=-1, S=0\right\rangle\left(\begin{array}{c|cc}
\frac{1}{2} & \frac{1}{2} & 0 \\
0+\mu & \mu & 0
\end{array}\right)=1
\end{aligned}
$$

Uma vez computados todos os invariantes e os autovalores de $H_{-1}$, a iteração $N=-1$ é fechada. Prosseguimos com a iteração $N=0$, que inicia o ciclo iterativo até o máximo valor $N$ satisfazendo a equação (3.33).

Como mencionamos anteriormente, qualquer iteração $N$ do ciclo pode ser construída a partir de cálculos da iteração $N-1$, pois os Hamiltonianos $H_{N}$ e $H_{N-1}$ estão relacionados pela transformação (3.35). Seja $\left\{\left|Q, S, S_{z}, p\right\rangle\right\}_{N}$ a base de estados da $N$-ésima, segue que 


$$
\begin{aligned}
\left\langle Q^{\prime}, S^{\prime}, S_{z}^{\prime}, p^{\prime}\left|H_{N}\right| Q, S, S_{z}, p\right\rangle= & \sqrt{\Lambda}\left\langle Q^{\prime}, S^{\prime}, S_{z}^{\prime}, p^{\prime}\left|H_{N-1}\right| Q, S, S_{z}, p\right\rangle \\
& +\frac{t_{N-1}}{\mathcal{D}_{N}}\left\langle Q^{\prime}, S^{\prime}, S_{z}^{\prime}, p^{\prime}\right|\left(f_{N-1}^{\dagger} f_{N}+\text { H.c. }\right)\left|Q, S, S_{z}, p\right\rangle .
\end{aligned}
$$

Em particular, para $N=0$, a equação (3.52) explicita a forma como $H_{0}$ pode ser construído a partir dos resultados da iteração $N=-1$. A seguir, mostraremos como construir a base $\left\{\left|Q, S, S_{z}, p\right\rangle\right\}_{N}$ a partir da base $\left\{\left|Q, S, S_{z}, p\right\rangle\right\}_{N-1}$ e demonstraremos como a nova base permite determinar a matriz Hamiltoniano $H_{N}(N>0)$ por meio dos autovalores $\lambda_{N-1}$ e dos elementos de matriz $\left\langle q^{\prime}, s^{\prime}, s_{z}^{\prime}\left|f_{N}^{\dagger}\right| q^{\prime}, s^{\prime}, s_{z}^{\prime}\right\rangle$.

\subsubsection{Setores $Q, S$ e base primitiva}

Como vimos na seção 3.6, as configurações eletrônicas possíveis para uma dada iteração $N$ definem os pares $(Q, S)$ permitidos pela adição de carga e spin, bem como a base de estados de $H_{N}$. Cada valor de carga $Q$ está associada a um conjunto definido de spins $[S]=[S, S+1, S+2, \ldots]$, assim como para um valor de spin $S$ temos o conjunto de cargas $[Q]=[Q, Q+1, Q+2, \ldots]$. Em princípio, a paridade da carga máxima $Q_{\max }^{N}$ da iteração $N$ fica condicionada à paridade da iteração, ou seja, para $N$ par segue que $Q_{\max }^{N}$ é par e para $N$ ímpar a carga $Q_{\max }^{N}$ é ímpar. Em geral, $Q_{\max }^{N}=N+2$.

Nesse sentido, podemos identificar os setores $(Q, S)$ ativos e inativos da iteração $N$ com casas pretas e brancas de um tabuleiro de xadrez, onde as linhas correspondem à carga $Q$ e as colunas ao spin $S$. Embora pictoricamente conveniente, esta analogia não é totalmente perfeita, uma vez que nem todas as casas de uma linha $Q$ possíveis no jogo real são ativas em uma iteração $N$, valendo o mesmo para as colunas $S$. Na prática, fixamos o número máximo de linhas $Q_{M A X}$ e colunas $S_{M A X}$ do tabuleiro, de modo que quando $Q_{\max }^{N}>Q_{M A X}$ a carga máxima da iteração $N$ pode ser $Q_{M A X}$ ou $Q_{M A X}-1$. As casas ativas da iteração $N$ formam um triângulo cuja base está na coluna $S=0$. Na figura 3.7 ilustramos os setores $(Q, S)$ ativos na iteração $N=6 \operatorname{com} Q_{M A X}=7$ e $S_{M A X}=7$.

No início da iteração $N(N=0,1, \ldots)$ são conhecidos o conjunto de autovalores $\lambda_{N-1}^{Q, S, l}$ $(l=1, \ldots, L(Q, S))$ (onde $L(Q, S)$ denota a dimensão do subespaço $(Q, S)$ na iteração $N-1$ ) e os invariantes $\left\langle Q+1, S^{\prime}, l^{\prime}\left\|f_{N-1}^{\dagger}\right\| Q, S, l\right\rangle_{N-1}$, com $S^{\prime}=S \pm 1 / 2, l=1, \ldots, L(Q, S)$ e

†Considerando a cadeia $\left\{f_{n}\right\}$ como infinita, sem restringir a carga máxima e o spin máximo para todo o ciclo iterativo. 
$l^{\prime}=1, \ldots, L\left(Q+1, S^{\prime}\right)$.

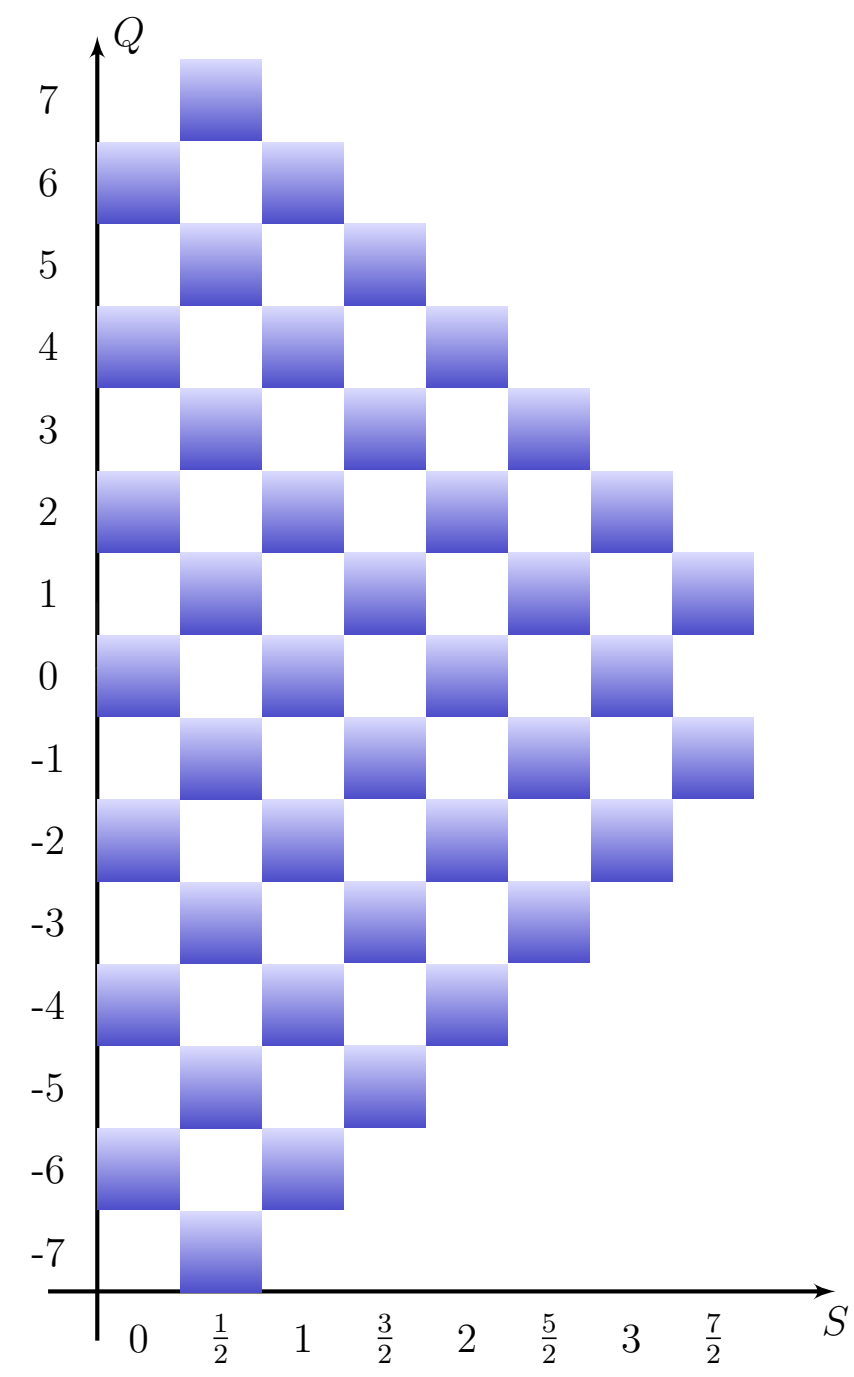

Figura 3.7 - Setores ativos (em azul) para a iteração $N=6$. A carga máxima é $Q_{\max }^{5}=7$. Para $Q=0$, o conjunto de spins permitidos é $[0,1,2,3]$, enquanto para $Q=7$ só é permitido $S=1$. Em uma iteração par setores de carga par têm spins pares e setores de carga ímpar têm spins ímpares. Fonte: elaborada pela autora.

A cada iteração $N$ introduzimos o operador $f_{N}$ no sistema. Pictoricamente, introduzir $f_{N}$ representa adicionar um novo sítio ao sistema em $N-1$, como ilustrado nas figuras 3.4 e 3.5. No contexto do tabuleiro de setores $(Q, S)$, tal operação corresponde a uma inversão de cores, ou seja, os setores ativos em $N-1$ tornam-se inativos em $N$. Ambas representações permitem relacionar a base de estados entre as iterações $N$ e $N-1$.

Dado um autovetor $\left|Q, S, S_{z}, l\right\rangle_{N-1}$ de $H_{N-1}$, geramos quatro bases de estados $\left|q, s, s_{z}, p\right\rangle_{N}$ 
com carga, spin e componente $z$ de spin bem determinados, através das relações

$$
\begin{aligned}
\left|Q-1, S, S_{z}, p_{3}\right\rangle_{N}^{\mathcal{S}} & =O_{\mathcal{S}}\left|Q, S, S_{z}, l\right\rangle_{N-1}, \\
\left|Q, S-1 / 2, S_{z}-1 / 2, p_{4}\right\rangle_{N}^{\mathcal{W}} & =O_{\mathcal{W}}\left|Q, S, S_{z}, l\right\rangle_{N-1}, \\
\left|Q, S+1 / 2, S_{z}+1 / 2, p_{2}\right\rangle_{N}^{\mathcal{E}} & =O_{\mathcal{E}}\left|Q, S, S_{z}, l\right\rangle_{N-1} \\
\left|Q+1, S, S_{z}, p_{1}\right\rangle_{N}^{\mathcal{N}} & =O_{\mathcal{N}}\left|Q, S, S_{z}, l\right\rangle_{N-1}
\end{aligned}
$$

onde os operadores $O_{g}(g=\mathcal{S}, \mathcal{W}, \mathcal{E}, \mathcal{N})$ são definidos pelas identidades

$$
\begin{aligned}
& O_{\mathcal{S}}\left|Q, S, S_{z}, l\right\rangle_{N-1} \equiv\left|Q, S, S_{z}, l\right\rangle_{N-1} \\
& O_{\mathcal{W}}\left|Q, S, S_{z}, l\right\rangle_{N-1} \equiv\left(\begin{array}{c|cc}
S-\frac{1}{2} & \frac{1}{2} & S \\
S_{z}-\frac{1}{2} & \frac{1}{2} & S_{z}-1
\end{array}\right) f_{N \uparrow}^{\dagger}\left|Q, S, S_{z}, l\right\rangle_{N-1} \\
& +\left(\begin{array}{c|cc}
S-\frac{1}{2} & \frac{1}{2} & S \\
S_{z}-\frac{1}{2} & -\frac{1}{2} & S_{z}
\end{array}\right) f_{N \downarrow}^{\dagger}\left|Q, S, S_{z}, l\right\rangle_{N-1} \\
& O_{\mathcal{E}}\left|Q, S, S_{z}, l\right\rangle_{N-1} \equiv \quad\left(\begin{array}{c|cc}
S+\frac{1}{2} & \frac{1}{2} & S \\
S_{z}+\frac{1}{2} & \frac{1}{2} & S_{z}
\end{array}\right) f_{N \uparrow}^{\dagger}\left|Q, S, S_{z}, l\right\rangle_{N-1} \\
& +\left(\begin{array}{c|cc}
S+\frac{1}{2} & \frac{1}{2} & S \\
S_{z}+\frac{1}{2} & -\frac{1}{2} & S_{z}+1
\end{array}\right) f_{N \downarrow}^{\dagger}\left|Q, S, S_{z}, l\right\rangle_{N-1} \\
& O_{\mathcal{N}}\left|Q, S, S_{z}, l\right\rangle_{N-1} \equiv f_{N \uparrow}^{\dagger} f_{N \downarrow}^{\dagger}\left|Q, S, S_{z}, l\right\rangle_{N-1} \text {. }
\end{aligned}
$$

Dizemos que os estados $\left|Q, S, S_{z}, l\right\rangle_{N-1}$ são os pais (parent) dos quatro estados filhos (child) e associamos a cada estado filho um genero $g=\mathcal{S}, \mathcal{W}, \mathcal{E}$ e $\mathcal{N}$, tal como apresentamos nas equações (3.53)-(3.56). Os rótulos $g=\mathcal{S}, \mathcal{W}, \mathcal{E}$ e $\mathcal{N}$ são atribuídos em analogia às coordenadas geográficas sul (south), oeste (west), leste (east) e norte (north) que direcionam estados dos setores pais - ativos na iteração $N-1$ - para os filhos da iteração $N$. Na figura 3.8 ilustramos como a base de $(Q, S)$ dá origem aos filhos $(q-1, s),(q, s+1 / 2),(q+1, s)$ e $(q, s-1 / 2)$.

Como cada setor filho $(q, s)$ herda estados de 4 setores pais nas direções $\mathcal{S}, \mathcal{W}, \mathcal{E}$ e $\mathcal{N}$, sua dimensão primitiva $n_{p}$ é dada pela soma

$$
n_{p}=L(q, s)=L(Q+1, S)+L\left(Q, S+\frac{1}{2}\right)+L(Q-1, S)+L\left(Q, S-\frac{1}{2}\right) .
$$

Dizemos que a base $|Q, S, p\rangle$ e a dimensão $n_{p}$ são primitivas por referirem-se aos estados 
existentes antes do corte ultravioleta. Após a diagonalização, somente os $n_{r}$ autovetores (e estados) associados aos autovalores $\lambda_{i}<E_{U V}$ são mantidos, de modo que $L(q, s)=n_{r}$.
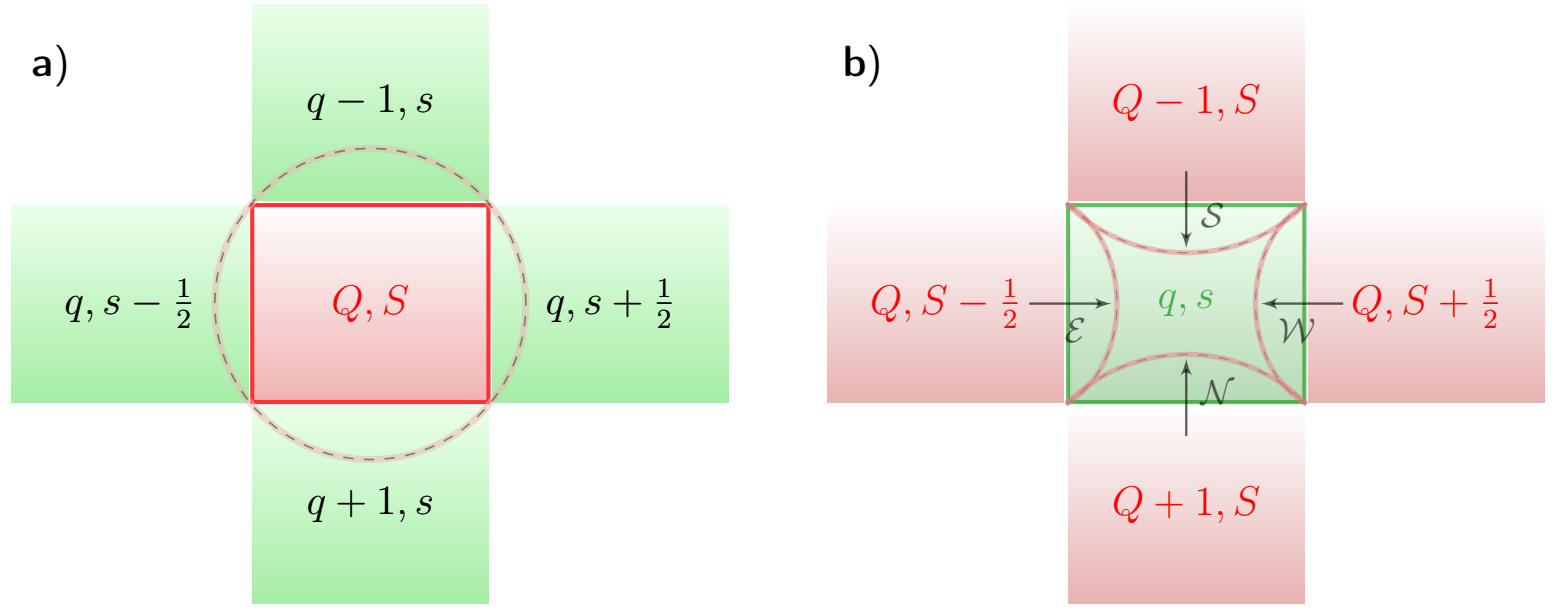

Figura 3.8 - Construção da base primitiva de um setor $(Q, S)$ na iteração $N$ a partir dos estados da iteração $N-1$. Setores pais e filhos são ilustrados em vermelho e verde, respectivamente. a) Seja $\left\{\left|Q, S, S_{z}, l\right\rangle\right\}$ a base de estados do setor $(Q, S)$ da iteração $N-1$. A atuação dos os operadores $O_{g}(g=\mathcal{S}, \mathcal{W}, \mathcal{E}, \mathcal{N})$ nessa base dá origem aos estados filhos nos respectivos setores $(q-1, s),(q, s+1 / 2),(q+1, s)$ e $(q, s-1 / 2)$ na iteração $N$. Os gêneros atribuídos aos operadores $O_{g}$ correspondem à localização geográfica dos setores filhos de $Q, S$ : norte $(\mathcal{N})$, leste $(\mathcal{E})$, sul $(\mathcal{S})$ e oeste $(\mathcal{W})$. b) A base primitiva de um setor filho $(q, s)$ na iteração $N$ é implementada através da herança de estados dos setores pais oriundos da iteração $N-1$. O setor $q, s$ recebe estados dos setores $(Q-1, S)$ via $O_{\mathcal{S}},(Q, S+1 / 2)$ via $O_{\mathcal{W}},(Q+1, S)$ via $O \mathcal{N}$ e $(Q, S-1 / 2)$ via $O_{\mathcal{W}}$ Fonte: elaborada pela autora.

Na figura 3.9 ilustramos a herança de estados entre os setores das iterações $N=-1 \mathrm{e}$ $N=0$. Partindo dos setores $(Q=1, S=0),(Q=0, S=1 / 2)$ e $(Q=-1, S=0)$ contendo um estado cada, obtemos um total de 36 estados os operadores $O_{g}(g=\mathcal{S}, \mathcal{W}, \mathcal{E}, \mathcal{N})$. Esses 36 estados filhos estão distribuídos entre os setores $(q=2, s=0),\left(q=1, s=\frac{1}{2}\right),(q=0, s=0)$, $\left(q=-1, s=\frac{1}{2}\right)$ e $(q=-2, s=0)$.

Em resumo, operamos com $O_{g}(g=\mathcal{S}, \mathcal{W}, \mathcal{E}, \mathcal{N})$ em todos os estados calculados na iteração $N-1$ para construir a base de estados da iteração $N$, na qual o Hamiltoniano (3.34) será projetado.

Para implementar a projeção no código NRG, basta notar que $O_{\mathcal{S}}$ e $O_{\mathcal{N}}\left(O_{\mathcal{W}}\right.$ e $\left.O_{\mathcal{E}}\right)$ comutam (anticomutam) com $f_{N-1 \mu}$. Desse modo, apenas o elemento $\sqrt{\Lambda} H_{N-1}$ da transformação (3.35) contribui para os elementos diagonais da matriz Hamiltoniano $H_{N}$, enquanto que os demais termos estão relacionados aos elementos não-diagonais. 

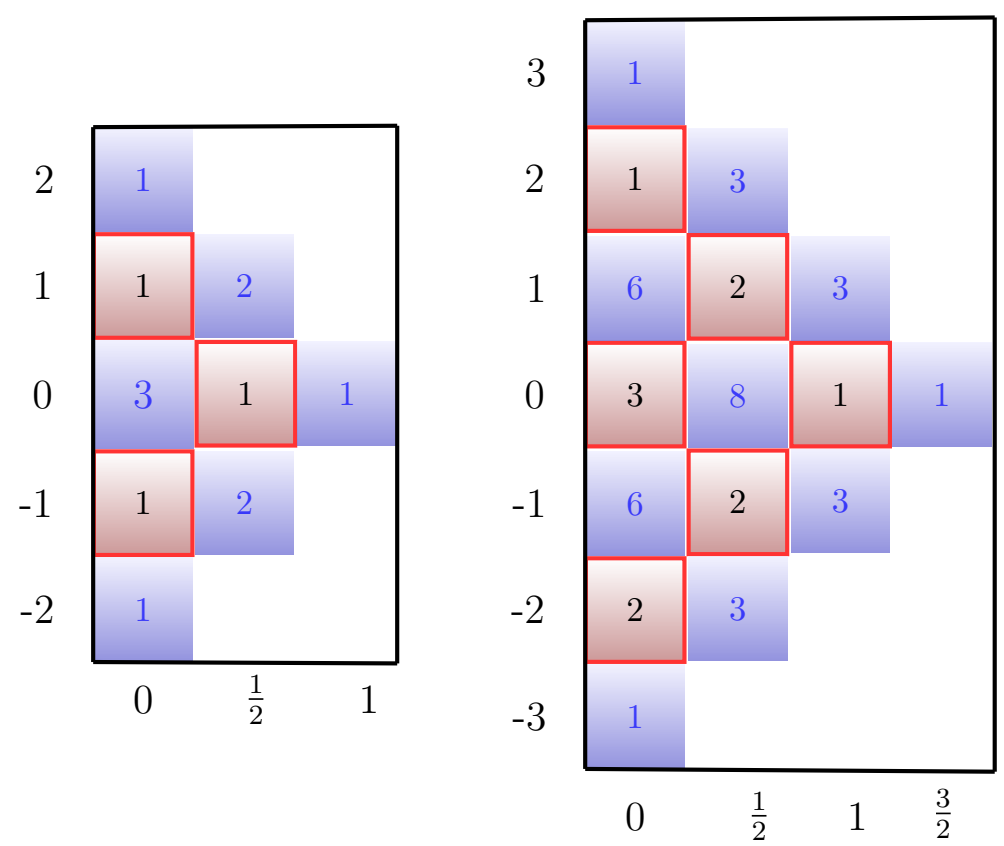

Figura 3.9 - Setores pais (vermelho) e filhos (azul) nas iterações $N=-1$ e $N=0$. A iteração $N=-1$ é composta pelos setores $(Q=1, S=0),\left(Q=0, S=\frac{1}{2}\right)$ e $(Q=-1, S=0)$, cada um contendo um estado. Esses estados são herdados na iteração $N=0$, dando origem à base primitiva dos setores $(Q=2, S=0),\left(Q=1, S=\frac{1}{2}\right),(Q=0, S=0)$, $\left(Q=-1, S=\frac{1}{2}\right)$ e $(Q=-2, S=0)$. Na iteração $N=1$, os setores filhos herdam os estados em $N=0$ cujos autovalores são inferiores a $E_{U V}$. Fonte: elaborada pela autora.

Os elementos da diagonal são simplesmente os autovalores computados na iteração anterior escalados pelo fator $\sqrt{\Lambda}$

$$
{ }^{g}\left\langle q, s, p\left|H_{N}\right| q, s, p\right\rangle^{g}=\sqrt{\Lambda} \lambda_{N-1}^{Q, S, l},
$$

onde $\lambda_{N-1}^{Q, S, l}(l=1, \ldots, L(Q, S))$ é o $l$-ésimo autovalor do setor $Q, S$ calculado na iteração $N-1$, enquanto $|Q, S, l\rangle_{N-1}$ é o estado pai de $|q, s, p\rangle_{N}^{g}$ com gênero $g$.

Cada elemento não-diagonal da matriz Hamiltoniano em um dado setor $Q, S$ pode ser fatorado em termos dos elementos de matriz dos operadores $f_{N}\left(f_{N}^{\dagger}\right)$ e de $f_{N-1}\left(f_{N-1}^{\dagger}\right)$

$$
\begin{gathered}
{ }^{g^{\prime}}\left\langle q, s, s_{z}, p\left|H_{N}\right| q, s,, s_{z}, p\right\rangle^{g}=\frac{t_{N-1}}{D_{N}}\left(\sum_{\mu} \alpha_{g^{\prime}, g}\left(S, S_{z}, \mu\right)\left\langle Q, S, S_{z}, l\left|f_{N-1 \mu}^{\dagger}\right| Q^{\prime}, S^{\prime}, S_{z}^{\prime}, l^{\prime}\right\rangle_{N-1}\right. \\
\left.+\sum_{\mu} \alpha_{g, g^{\prime}}\left(S, S_{z}, \mu\right)\left\langle Q^{\prime}, S^{\prime}, S_{z}^{\prime}, l^{\prime}\left|f_{N-1 \mu}\right| Q, S, S_{z}, l\right\rangle_{N-1}\right)
\end{gathered}
$$

onde $\left|Q, S, S_{z}, l\right\rangle_{N-1}$ é o pai de $\left|q, s, s_{z}, p\right\rangle_{N}^{g}$.

Os coeficientes $\alpha_{g, g^{\prime}}$ nas somas da eq. (3.63) são calculados a partir das identidades para os operadores $O_{g}(g=\mathcal{S}, \mathcal{W}, \mathcal{E}, \mathcal{N})$ dadas nas eqs. (3.57)-(3.60) Ao efetuarmos a soma 
sobre spins $\mu$ na eq. (3.63), obtemos uma expressão relacionando os elementos não-diagonais $\left\langle q, s, s_{z}, p^{\prime}\left|H_{N}\right| q, s, s_{z}, p\right\rangle$ aos invariantes da iteração $N-1$. Tal expressão, que depende somente dos gêneros de $\left|q, s, s_{z}, p^{\prime}\right\rangle$ e $\left|q, s, s_{z}, p\right\rangle$, é apresentada na tabela 3.1.

Assim como os elementos diagonais, os elementos não-diagonais são independentes de $S_{z}$ devido à invariância rotacional do Hamiltoniano. Na prática, a diagonalização do Hamiltoniano é feita sem referência a $S_{z}$.

Tabela 3.1 - Elementos não-diagonais da matriz Hamiltoniano $H_{N}$ entre os estados $\left|q, s, s_{z}, p^{\prime}\right\rangle_{N}^{g^{\prime}}$ e $\left|q, s, s_{z}, p\right\rangle_{N}^{g}$. Os fatores que aparecem na frente dos invariantes são os coeficientes de Clebsch-Gordan. As combinações não listadas de gêneros $g$ e $g^{\prime}$ são nulas.

\begin{tabular}{|c|c|c|}
\hline$g$ & $g^{\prime}$ & $g^{\prime}\left\langle q, s, s_{z}, p^{\prime}\left|H_{N}\right| q, s, s_{z}, p\right\rangle_{N}^{g}$ \\
\hline $\mathcal{E}$ & $\mathcal{N}$ & $\sqrt{\frac{2 s}{2 s+1}}\left\langle q, s-\frac{1}{2}, l^{\prime}\left\|f_{N-1}^{\dagger}\right\| q-1, s, l\right\rangle_{N-1}$ \\
\hline $\mathcal{W}$ & $\mathcal{N}$ & $-\sqrt{\frac{2 s+2}{2 s+1}}\left\langle q, s-\frac{1}{2}, l^{\prime}\left\|f_{N-1}^{\dagger}\right\| q-1, s, l\right\rangle_{N-1}$ \\
\hline $\mathcal{N}$ & $\mathcal{E}$ & $\sqrt{\frac{2 s}{2 s+1}}\left\langle q-1, s, l^{\prime}\left\|f_{N-1}^{\dagger}\right\| q, s-\frac{1}{2}, l\right\rangle_{N-1}$ \\
\hline $\mathcal{S}$ & $\mathcal{E}$ & $\left\langle q+1, s, l^{\prime}\left\|f_{N-1}^{\dagger}\right\| q, s-\frac{1}{2}, l\right\rangle_{N-1}$ \\
\hline $\mathcal{E}$ & $\mathcal{S}$ & $\left\langle q, s-\frac{1}{2}, l^{\prime}\left\|f_{N-1}^{\dagger}\right\| q+1, s, l\right\rangle_{N-1}$ \\
\hline $\mathcal{W}$ & $\mathcal{S}$ & $\left\langle q, s+\frac{1}{2}, l^{\prime}\left\|f_{N-1}^{\dagger}\right\| q+1, s, l\right\rangle_{N-1}$ \\
\hline $\mathcal{N}$ & $\mathcal{W}$ & $-\sqrt{\frac{2 s+2}{2 s+1}}\left\langle q-1, s, l^{\prime}\left\|f_{N-1}^{\dagger}\right\| q, s+\frac{1}{2}, l\right\rangle_{N-1}$ \\
\hline $\mathcal{S}$ & $\mathcal{W}$ & $\left\langle q+1, s, l^{\prime}\left\|f_{N-1}^{\dagger}\right\| q, s+\frac{1}{2}, l\right\rangle_{N-1}$ \\
\hline
\end{tabular}

Fonte: elaborada pela autora.

Recapitulando as etapas do ciclo apresentadas até a presente seção: para cada setor $(Q, S)$ os elementos de matriz diagonais de $H_{N}$ são obtidos a partir dos autovalores de $H_{N-1}$, como mostra a eq. (3.62). Os termos não-diagonais são calculados a partir dos elementos de matriz do operador $f_{N-1}^{\dagger}$ computados na iteração precedente. Uma vez construída, a matriz representando o Hamiltoniano $H_{N}$ em um dado setor $Q, S$ é diagonalizada, e seus autovalores e autovetores são armazenados. Os autovalores são mantidos até a iteração $N+1$. Os 
autovetores são temporariamente necessários, pois são utilizados para o cálculo dos elementos de matriz reduzidos do operador $f_{N}^{\dagger}$ na iteração $N$ atual. Detalharemos na próxima seção o cálculo desses elementos $\left\langle q, s, l\left\|f_{N}^{\dagger}\right\| q, s, l\right\rangle_{N}$.

\subsubsection{Cálculo dos invariantes}

A relação de Wigner-Eckart (3.48) mostra que os invariantes $\left\langle q+1, s^{\prime}, r^{\prime}\left\|f_{N}^{\dagger}\right\| q, s, r\right\rangle_{N}$ são facilmente calculáveis a partir dos elementos de matriz $\left\langle q^{\prime}, s^{\prime}, s_{z}^{\prime}, r^{\prime}\left|f_{N \mu}^{\dagger}\right| q, s, s_{z}, r\right\rangle_{N}(\mu=$ $\pm 1 / 2$ ). Os últimos estão relacionados com os elementos de matriz de $f_{N \mu}^{\dagger}$ na base dos estados filhos $\left|q, s, s_{z}, p\right\rangle_{N}^{g}$ pela igualdade

$$
\begin{aligned}
\left\langle q+1, s^{\prime}, s_{z}^{\prime}, r^{\prime}\left|f_{N \mu}\right| q, s, s_{z}, r\right\rangle_{N}= & \sum_{p, p^{\prime}} U_{r^{\prime} p^{\prime}}^{\dagger}\left(q+1, s^{\prime}\right) U_{r p}(q, s) \\
& \times^{g^{\prime}}\left\langle q+1, s^{\prime}, s_{z}^{\prime}, p^{\prime}\left|f_{N \mu}^{\dagger}\right| q, s, s_{z}, p\right\rangle_{N}^{g},
\end{aligned}
$$

onde $U_{r p}(q, s)$ denota a transformação unitária que diagonaliza $H_{N}$ na base dos estados filhos $\left|q, s, s_{z}, p\right\rangle_{N}^{g}$. A transformação $U_{r p}(q, s)$ é a matriz composta pelos autovetores resultantes da diagonalização de $H_{N}$, onde cada coluna é um autovetor.

a)

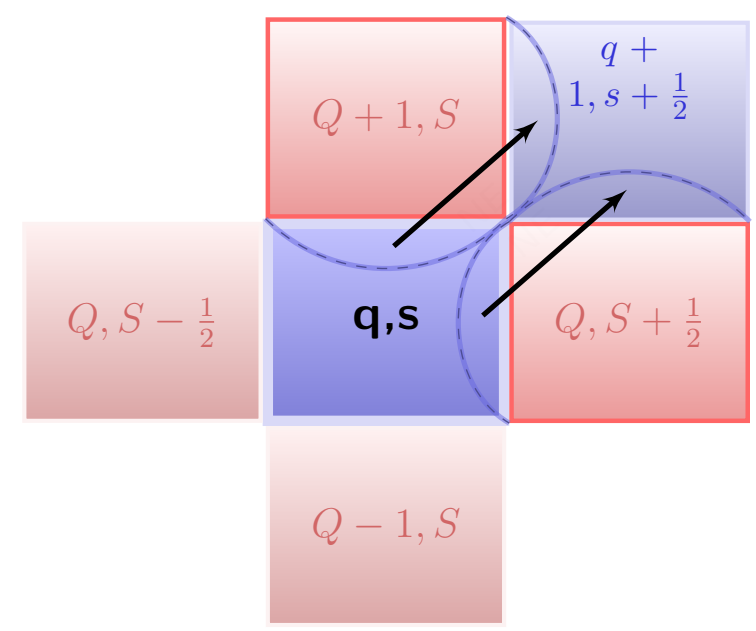

b)

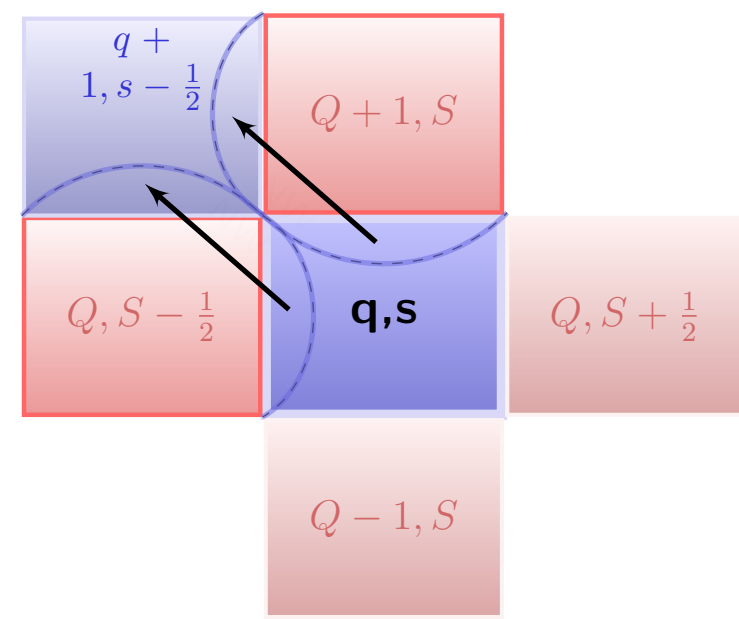

Figura 3.10 - Esquema de cálculo dos invariantes $N E$ e $N W$ de um setor $(Q, S)$ com os setores $\left(Q+1, S+\frac{1}{2}\right)$ e $\left(Q+1, S-\frac{1}{2}\right)$, respectivamente. Os setores circulados em vermelho correspondem aos pais de $(Q, S)$ existentes na iteração anterior. a) Os invariantes nordeste $\left\langle q+1, s+1 / 2, r^{\prime}|| f_{N} \| q, s, r\right\rangle$ recebem a contribuição de estados com origem nos setores pais $(Q+1, S)$ e $\left(Q, S+\frac{1}{2}\right)$. b) Os invariantes noroeste $\left\langle q+1, s-1 / 2, r^{\prime}\left\|f_{N}\right\| q, s, r\right\rangle$ recebem a contribuição de estados com origem nos setores pais $(Q+1, S)$ e $\left(Q, S-\frac{1}{2}\right)$. Fonte: elaborada pela autora. 
Lembrando que os operadores $f_{N \mu}$ aniquilam os autoestados $\left|Q, S, S_{z}, r\right\rangle_{N-1}$, é relativamente simples computar cada um dos elementos de matriz da equação (3.64) a partir das relações (3.53)-(3.56). Os elementos de matriz são nulos a menos que os estados $\left|q+1, s^{\prime}, s_{z}+\mu, p^{\prime}\right|_{N}^{g^{\prime}}\left|q, s, s_{z}, p\right\rangle_{N}$ tenham um pai comum, ou seja, a menos que verifiquemse as relações

$$
\left|q, s, s_{z}, p\right\rangle_{N}^{g}=O_{g}\left|Q, S, S_{z}, r\right\rangle_{N-1}
$$

e

$$
\left|q^{\prime}, s^{\prime}, s_{z}+\mu, p\right\rangle_{N}^{g^{\prime}}=O_{g^{\prime}}\left|Q, S, S_{z}, r\right\rangle_{N-1},
$$

com gêneros $g$ e $g^{\prime}$ tais que $q^{\prime}=q+1$ e $s^{\prime}=s \pm 1 / 2$.

Com as equações (3.65) e (3.66), concluímos que os elementos de matriz não se anulam para as combinações de gêneros $\mathcal{N} \mathcal{W}, \mathcal{E S}, \mathcal{N E}$ e $\mathcal{W S}$. Apresentados na tabela 3.2 os resultados de ${ }^{\prime}\left\langle q+1, s^{\prime}, s_{z}+\mu, p^{\prime}\left|f_{N \mu}^{\dagger}\right| q, s, s_{z}, p\right\rangle_{N}^{g}$ para esses pares de gêneros.

Tabela 3.2 - Elementos de matriz ${ }^{\prime}\left\langle q+1, s^{\prime}, s_{z}+\mu, p^{\prime}\left|f_{N \mu}^{\dagger}\right| q, s, s_{z}, p\right\rangle_{N}^{g}$ não nulos a partir dos quais os invariantes são computados. A última coluna apresenta os coeficientes de Clebsch-

\begin{tabular}{|c|c|c|c|}
\hline$s^{\prime}$ & $g$ & $g^{\prime}$ & $g^{\prime}\left\langle q+1, s^{\prime}, s_{z}+\mu, p^{\prime}\left|f_{N \mu}^{\dagger}\right| q, s, s_{z}, p\right\rangle_{N}^{g}$ \\
\hline$s+\frac{1}{2}$ & $\mathcal{N}$ & $\mathcal{W}$ & $2 \mu\left(\begin{array}{c|cc}s & \frac{1}{2} & s+\frac{1}{2} \\
s_{z} & -\mu & s_{z}+\mu\end{array}\right)$ \\
\hline$s+\frac{1}{2}$ & $\mathcal{E}$ & $\mathcal{S}$ & $\left(\begin{array}{c|cc}s+\frac{1}{2} & \frac{1}{2} & s \\
s_{z}+\mu & \mu & s_{z}\end{array}\right)$ \\
\hline$s-\frac{1}{2}$ & $\mathcal{N}$ & $\mathcal{E}$ & $2 \mu\left(\begin{array}{c|cc}s & \frac{1}{2} & s-\frac{1}{2} \\
s_{z} & -\mu & s_{z}+\mu\end{array}\right)$ \\
\hline$s-\frac{1}{2}$ & $\mathcal{W}$ & $\mathcal{S}$ & $\left(\begin{array}{c|cc}s-\frac{1}{2} & \frac{1}{2} & s \\
s_{z}+\mu & \mu & s_{z}\end{array}\right)$ \\
\hline
\end{tabular}
Gordan para as combinações de gêneros que produzem elementos diferentes de zero.

Fonte: elaborada pela autora.

É possível derivar outra simplificação no cálculo dos invariantes, observando que os coeficientes de Clebsch-Gordan na primeira e segunda linhas da última coluna da tabela 3.2 são 
relacionados pela expressão

$$
\left(\begin{array}{c|cc}
s & \frac{1}{2} & s+\frac{1}{2} \\
s_{z} & -\mu & s_{z}+\frac{1}{2}
\end{array}\right)=2 \mu \sqrt{\frac{2 s+1}{2 s+1}}\left(\begin{array}{c|cc}
s+\frac{1}{2} & \frac{1}{2} & s \\
s_{z}+\mu & \mu & s_{z}
\end{array}\right)
$$

enquanto os coeficientes da terceira e da quarta linha estão relacionados por

$$
\left(\begin{array}{c|cc}
s & \frac{1}{2} & s-\frac{1}{2} \\
s_{z} & -\mu & s_{z}+\frac{1}{2}
\end{array}\right)=2 \mu \sqrt{\frac{2 s+1}{2 s}}\left(\begin{array}{c|cc}
s-\frac{1}{2} & \frac{1}{2} & s \\
s_{z}+\mu & \mu & s_{z}
\end{array}\right) .
$$

Desse modo, é conveniente definir uma matriz $\mathcal{M}_{g, g^{\prime}}\left(g, g^{\prime}=\mathcal{N}, \mathcal{E}, \mathcal{S}, \mathcal{W}\right)$ de dimensão $4 \times 4$, cujos elementos não nulos são:

$$
\begin{aligned}
& \mathcal{M}_{\mathcal{N}, \mathcal{W}}=\sqrt{\frac{2 s+1}{2 s+2}} \\
& \mathcal{M}_{\mathcal{E}, \mathcal{S}}=1 \\
& \mathcal{M}_{\mathcal{N}, \mathcal{W}}=-\sqrt{\frac{2 s+1}{2 s}} \\
& \mathcal{M}_{\mathcal{W}, \mathcal{S}}=1 .
\end{aligned}
$$

Com as definições (3.69)-(3.72), a tabela 3.2 pode ser sumarizada na equação

$$
{ }^{g^{\prime}}\left\langle q+1, s \pm \frac{1}{2}, s_{z}+\mu, r^{\prime}\left|f_{N \mu}^{\dagger}\right| q, s, s_{z}, r\right\rangle_{N}^{g}=\left(\begin{array}{c|cc}
s \pm \frac{1}{2} & \frac{1}{2} & s \\
s_{z}+\mu & \mu & s_{z}
\end{array}\right) \mathcal{M}_{g, g^{\prime}}
$$

Substituindo a eq. (3.73) em (3.64), obtemos uma expressão simples para os elementos de matriz de $f_{N \mu}^{\dagger}$ na base de autoestados de $H_{N}$

$$
\begin{aligned}
\left\langle q+1, s \pm \frac{1}{2}, s_{z}+\mu, r^{\prime}\left|f_{N \mu}^{\dagger}\right| q, s, s_{z}, r\right\rangle_{N}= & \left(\begin{array}{c|cc}
s \pm \frac{1}{2} & \frac{1}{2} & s \\
s_{z}+\mu & \mu & s_{z}
\end{array}\right) \\
& \times \sum_{p, p^{\prime}} U_{r^{\prime} p^{\prime}}^{\dagger}\left(q+1, s^{\prime}\right) \mathcal{M}_{g, g^{\prime}} U_{r p}(q, s)
\end{aligned}
$$

onde $g$ e $g^{\prime}$ são os gêneros do $p$-ésimo e $p^{\prime}$-ésimo estados filhos dos setores $q, s$ e $q+1, s \pm \frac{1}{2}$, respectivamente. 
Comparando a última equação com relação de Wigner-Eckart em (3.48), podemos então calcular os invariantes através da expressão

$$
\left\langle q+1, s \pm \frac{1}{2}, r^{\prime}\left\|f_{N}^{\dagger}\right\| q, s, r\right\rangle_{N}=\sum_{p, p^{\prime}} U_{r^{\prime} p^{\prime}}^{\dagger}\left(q+1, s^{\prime}\right) \mathcal{M}_{g, g^{\prime}} U_{r p}(q, s)
$$

a)

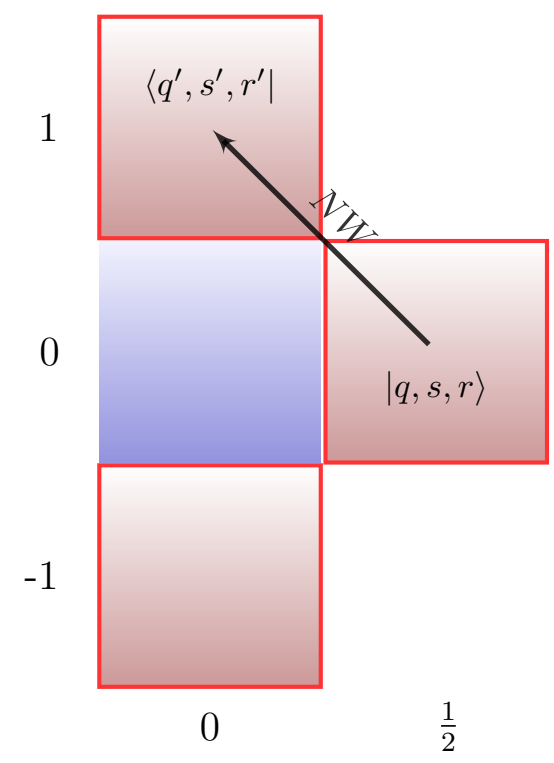

b)

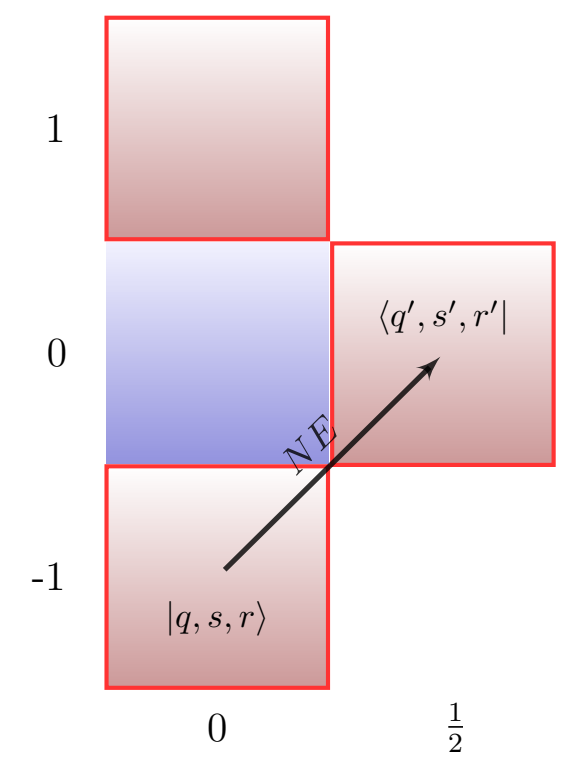

Figura 3.11 - Cálculo dos invariantes entre os estados da iteração $N=-1$. a)Os estados $|Q=0, S=1 / 2,1\rangle$ e $|Q=1, S=0,1\rangle$ juntos compõe o elemento de matriz $\left\langle Q=1, S=0, r^{\prime}=1\left\|f_{-1}\right\| Q=0, S=1 / 2, r=1\right\rangle$ na direção $N W$ do setor $(Q=$ $0, S=1 / 2)$. b)Por sua vez, o invariante $N E$ do setor $(Q=-1, S=0)$ é construído a partir dos estados $|Q=-1, S=0, r=1\rangle$ e $\left|Q=0, S=1 / 2, r^{\prime}=1\right\rangle$. Fonte: elaborada pela autora.

A última passagem nos diz que a soma em $p$ e $p^{\prime}$ é restrita a estados que obedeçam às relações (3.65) e (3.66). Na prática, se $L(Q, S)$ é a dimensão do setor $Q, S$ a partir dos quais foram gerados os estados filhos $\left|q, s, s_{z}, p\right\rangle_{N}$ e $\left|q+1 s \pm 1 / 2, s_{z}+\mu, p^{\prime}\right\rangle_{N}$, então a soma que aparece à direita na eq. (3.75) engloba $L$ termos, e não $L^{2}$ como a soma dupla poderia sugerir. Portanto, o custo para computar $L^{2}$ invariantes à esquerda da eq. (3.75) é proporcional a $L^{3}$.

Seguindo o procedimento detalhado na presente seção, computamos e armazenamos os invariantes $\left\langle q^{\prime}, s^{\prime}, r^{\prime}\left\|f_{N}^{\dagger}\right\| q, s, r\right\rangle$ em cada iteração $N$. Em seguida, prosseguimos calculando propriedades físicas, tais como a condutância, a densidade espectral ou mesmo a taxa de 
relaxação magnética. Tais propriedades dependem essencialmente de elementos de matriz na forma $\left\langle q^{\prime}, s^{\prime}, r^{\prime}\left|c_{d \mu}^{\dagger}\right| q, s, r\right\rangle$ e $\left\langle q^{\prime}, s^{\prime}, r^{\prime}\left|f_{n \mu}^{\dagger}\right| q, s, r\right\rangle(n=0, \ldots, N)$.

Para economizar memória, mantemos armazenados somente os invariantes, uma vez a partir deles podemos obter os elementos não-diagonais do Hamiltoniano nas iterações subsequentes e ainda calcular os demais elementos de matriz úteis às propriedades de interesse. Na próxima seção, detalharemos a última etapa do ciclo iterativo, que compreende o cálculo recursivo dos elementos de matriz reduzidos $\left\langle q^{\prime}, s^{\prime}, r^{\prime}\left|f_{n \mu}^{\dagger}\right| q, s, r\right\rangle(n=-1,0, \ldots, N){ }^{\ddagger}$ para a obtenção de propriedades físicas.

\subsubsection{Cálculo recursivo dos elementos de matriz}

A cada iteração $N$, estaremos interessados no comportamento de propriedades de excitação ou transporte, que podem variar com a energia ou com a temperatura do sistema. Em geral, um observável $\mathcal{X}$ associado à grandeza física $\chi$ é escrito como

$$
\chi \propto \sum_{\varphi, \psi}\left|\left\langle\varphi\left|f_{n}\right| \psi\right\rangle\right|^{2} f(T, \varepsilon)
$$

com $n=-1,0, \ldots, N,|\varphi\rangle$ e $|\psi\rangle$ sendo autoestados do Hamiltoniano correspondente e $f(T, \varepsilon)$ é uma função que pode depender da temperatura $T$ ou das energias $\varepsilon$.

Como mencionamos na seção 3.6.3, é conveniente manter em memória apenas os invariantes $\left\langle q^{\prime}, s^{\prime}, r^{\prime}\left\|f_{N}^{\dagger}\right\| q, s, r\right\rangle$ e a partir deles resgatar os demais elementos $\left\langle q^{\prime}, s^{\prime}, r^{\prime}\left\|f_{n}^{\dagger}\right\| q, s, r\right\rangle$ da cadeia $\left\{f_{n}\right\}$. Para tanto, precisamos encontrar uma relação envolvendo os $f_{n} \operatorname{com}$ os $f_{N}$ o que mostramos a seguir.

Iniciamos calculando o comutador de $H_{N}$ com o operador $f_{n}^{\dagger}$

$$
\begin{aligned}
{\left[H_{N}, f_{n}^{\dagger}\right] } & =\frac{1}{\mathcal{D}_{N}} \sum_{p} t_{p}\left(\left[f_{p}^{\dagger} f_{p+1}, f_{n}^{\dagger}\right]+\left[f_{p+1}^{\dagger} f_{p}, f_{n}^{\dagger}\right]\right) \\
& =\frac{1}{\mathcal{D}_{N}} \sum_{p} t_{p} f_{p}^{\dagger} \delta_{p+1, n}+\frac{1}{\mathcal{D}_{N}} \sum_{p} t_{p} f_{p+1}^{\dagger} \delta_{n, p} \\
& =\frac{t_{n-1}}{\mathcal{D}_{N}} f_{n-1}^{\dagger}+\frac{t_{n}}{\mathcal{D}_{N}} f_{n+1}^{\dagger} .
\end{aligned}
$$

¥O índice $n=-1$ é utilizado como notação para indicar o elemento de matriz do operador $c_{d}$, ou seja, $\left\langle q^{\prime}, s^{\prime}, r^{\prime}\left|f_{-1 \mu}^{\dagger}\right| q, s, r\right\rangle=\left\langle q^{\prime}, s^{\prime}, r^{\prime}\left|c_{d \mu}^{\dagger}\right| q, s, r\right\rangle$. 
Em particular, para $n=N$

$$
\left[H_{N}, f_{N}^{\dagger}\right]=\frac{t_{N-1}}{\mathcal{D}_{N}} f_{N-1}^{\dagger}
$$

de modo que o elemento de matriz do comutador é dado por

$$
\begin{aligned}
\frac{t_{N-1}}{\mathcal{D}_{N}}\left\langle q+1, s \pm \frac{1}{2}, r^{\prime}\left\|f_{N-1}^{\dagger}\right\| q, s, r\right\rangle_{N}= & \left\langle q+1, s \pm \frac{1}{2}, r^{\prime}\left\|H_{N} f_{N}^{\dagger}\right\| q, s, r\right\rangle_{N} \\
& -\left\langle q+1, s \pm \frac{1}{2}, r^{\prime}\left\|f_{N}^{\dagger} H_{N}\right\| q, s, r\right\rangle_{N} .
\end{aligned}
$$

Uma vez que os estados $|q, s, r\rangle_{N}$ e $\left|q+1, s \pm \frac{1}{2}, r^{\prime}\right\rangle_{N}$ são autoestados de $H_{N}$ com autovalores $E_{N}(q, s, r)$ e $E_{N}\left(q+1, s \pm \frac{1}{2}, r^{\prime}\right)$, respectivamente, a equação (3.79) pode ser reescrita como

$$
\frac{t_{N-1}}{\mathcal{D}_{N}}\left\langle q+1, s \pm \frac{1}{2}, r^{\prime}\left\|f_{N-1}^{\dagger}\right\| q, s, r\right\rangle_{N}=\Delta E_{N}\left\langle q+1, s \pm \frac{1}{2}, r^{\prime}\left\|f_{N}^{\dagger}\right\| q, s, r\right\rangle_{N},
$$

onde

$$
\Delta E_{N}=E_{N}\left(q+1, s \pm \frac{1}{2}, r^{\prime}\right)-E_{N}(q, s, r),
$$

é a diferença energética entre os estados $r^{\prime}$ e $r$.

A relação (3.80) determina os elementos de matriz de $f_{N-1}^{\dagger}$ entre quaisquer pares de estados $|q, s, r\rangle$ e $\left|q+1, s \pm \frac{1}{2}, r^{\prime}\right\rangle$.

Em seguida, gostaríamos de calcular os elementos de matriz de $f_{n}^{\dagger}$ com $n=N-2, N-$ $1, \ldots, 0$ de maneira análoga. Assim, computamos o comutador $\left[H_{N}, f_{n+1}^{\dagger}\right]$

$$
\left[H_{N}, f_{n+1}^{\dagger}\right]=\frac{1}{\mathcal{D}_{N}}\left(t_{n} f_{n}^{\dagger}+t_{n+1} f_{n+2}^{\dagger}\right)
$$

seguindo que

$$
\begin{aligned}
\left\langle q+1, s \pm \frac{1}{2}, r^{\prime}\left\|f_{n}^{\dagger}\right\| q, s, r\right\rangle_{N}= & -\frac{t_{n+1}}{t_{n}}\left\langle q+1, s \pm \frac{1}{2}, r^{\prime}\left\|f_{n+2}^{\dagger}\right\| q, s, r\right\rangle_{N} \\
& +\frac{\mathcal{D}_{N}}{t_{n}} \Delta E_{N}\left\langle q+1, s \pm \frac{1}{2}, r^{\prime}\left\|f_{n+1}^{\dagger}\right\| q, s, r\right\rangle_{N} .
\end{aligned}
$$

Finalmente, para calcular o elemento de matriz do operador $c_{d}$, devemos calcular o comutador $\left[H_{N}, f_{0}^{\dagger}\right]$

$$
\left[H_{N}, f_{0}^{\dagger}\right]=\frac{1}{\mathcal{D}_{N}}\left(\sqrt{2} V c_{d}^{\dagger} t_{0} f_{1}^{\dagger}\right)
$$


a partir da qual obtemos a seguinte expressão

$$
\begin{aligned}
\left\langle q+1, s \pm \frac{1}{2}, r^{\prime}\left\|c_{d}^{\dagger}\right\| q, s, r\right\rangle_{N}= & -\frac{t_{0}}{\sqrt{2} V}\left\langle q+1, s \pm \frac{1}{2}, r^{\prime}\left\|f_{1}^{\dagger}\right\| q, s, r\right\rangle_{N} \\
& +\frac{\mathcal{D}_{N}}{\sqrt{2} V} \Delta E_{N}\left\langle q+1, s \pm \frac{1}{2}, r^{\prime}\left\|f_{0}^{\dagger}\right\| q, s, r\right\rangle_{N} .
\end{aligned}
$$

A equação (3.83) define a recursão entre os elementos $\left\langle q+1, s \pm \frac{1}{2}, r^{\prime}\left\|f_{n}^{\dagger}\right\| q, s, r\right\rangle_{N}$ para $n=0, \ldots, N$. Calculados os elementos de matriz entre os operadores $f_{0}^{\dagger}$ e $f_{1}^{\dagger}$, é possível obter os invariantes de $c_{d}^{\dagger}$ conforme a equação (3.85). O custo computacional de calcular o conjunto de todos os elementos de matriz dos operadores $c_{d}^{\dagger}$ e $f_{n}^{\dagger}$ para cada um dos autoestados dos setores $(q, s)$ de dimensão $L(q, s)$ é da ordem de $O\left(N L^{2}\right)$, o que para matrizes $L>N$ é inferior do que a complexidade $O\left(L^{3}\right)$ da diagonalização de $H_{N}$ projetado no subespaço $(q, s)$.

Até o presente desenvolvimento, nosso problema foi tratado analiticamente com ferramentas conhecidas na literatura. Todos os conceitos introduzidos nos capítulos 2 e 3 foram essenciais para fundamentar o problema quanticamente. Fomos capazes de obter uma expressão para o Hamitolniano $H_{A}$ não-perturbado na base do NRG, enquanto o operador $H_{\text {probe }}$ representando a ponta de prova foi projetado na base contínua $\left\{c_{\varepsilon}, \bar{c}_{\varepsilon}\right\}$. No próximo capítulo, de número 4 , mostraremos como resolver $H_{\text {probe }}$ na base $\left\{f_{n}, \bar{c}_{\varepsilon}\right\}$, de modo que a taxa de relaxação $1 / T_{1}$ seja calculada numericamente. 


\section{A taxa de relaxação magnética longitudinal}

Nothing in life is to be feared, it is only to be understood. Now is the time to understand more, so that we may fear

less. Marie Curie

Nossa proposta de estudar a nuvem Kondo e determinar seu raio $R_{k}$ é baseada no cálculo perturbativo da taxa de relaxação longitudinal $1 / T_{1}(5,27)$ de uma ponta de prova magnética inserida no metal hospedeiro a uma distância $R$ da impureza.

A motivação para esta proposta é decorrente do interesse recente em técnicas experimentais para explorar propriedades magnéticas em sistemas Kondo, tal como espectroscopia de ressonância paramagnética eletrônica (ESR, electron spin ressonance) na medida da susceptibilidade $(28,49)$. Essa técnica é capaz de medir a largura de ressonância de absorção de sistemas com momento magnético bem definido. No caso especial de uma liga metálica típica para um sistema Kondo, as rápidas flutuações tornam o sinal ilegível, requerindo a introdução de uma ponta de prova adicional, usualmente $G d^{3+}$. Assim, consegue-se detectar indiretamente o efeito da susceptibilidade do íon de Kondo por meio da interação de troca $R K K Y$ transmitida através dos elétrons de condução. A largura de linha da prova com momento magnético estável é dada pela taxa de relaxação de spin $\left(1 / T_{2}\right)$, que em metais é igual a taxa longitudinal $\left(1 / T_{1}\right)$.

Vamos mostrar que o Hamiltoniano $H_{\text {probe }}$ na equação (2.26) possui três termos contribuindo para a taxa de relaxação $1 / T_{1}$, os quais denominaremos contribuições escalar, vetorial e matricial. O primeiro é uma constante associada aos operadores $\bar{c}_{\varepsilon}$. Os outros dois termos são dependentes da temperatura, sendo um deles associado aos operadores $f_{n}$, enquanto o outro corresponde à interferência entre os $\bar{c}_{\varepsilon}$ e $f_{n}$. 


\subsection{Calculando a relaxação de um sistema Kondo}

Objetivando estudar a relaxação magnética do sistema perturbado pela ponta de prova, devemos ser capazes de medir a taxa de transição do sistema entre estados quânticos eletrô-

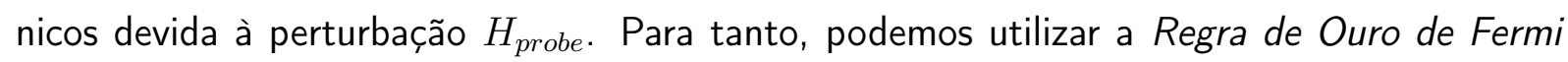
$(28,49)$ que permite calcular a taxa de relaxação magnética da ponta de prova através da expressão

$$
\frac{1}{T_{1}}=\frac{4 \pi}{\hbar} \sum_{I, F} P_{I}\left|\left\langle I\left|H_{\text {probe }}\right| F\right\rangle\right|^{2} \delta\left(E_{I}-E_{F}\right),
$$

onde $\beta=1 / k_{B} T$, e $|I\rangle$ e $|F\rangle$ são os estados inicial e final do Hamiltoniano (2.1) com energias $E_{I}$ e $E_{F}$, respectivamente e

$$
P_{I}=\frac{e^{-\beta E_{I}}}{\sum_{|I\rangle} e^{-\beta E_{I}}}
$$

onde são os pesos de Boltzmann dependentes da temperatura $T$.

A taxa $1 / T_{1}$ na equação (4.1) mede a probabilidade de transição entre os estados $|I\rangle$ e $|F\rangle$ do Hamiltoniano $H_{A}$ não-perturbado com pesos probabilísticos dados em (4.2). Em experimentos de ressonância magnética, as taxas de relaxação são medidas através da resposta do sistema a um campo pulsado com frequência $\omega$ (o que, no nosso caso, equivale a ter $H_{\text {probe }}$ dependente do tempo). Mais adiante, vamos discutir como física envolvida na transição mediada por $H_{\text {probe }}$ pode incorporada na solução analítica da soma em (4.1).

O cálculo de $1 / T_{1}$ em (4.1) requer essencialmente a projeção do Hamiltoniano $H_{\text {probe }}$ na base de autoestados do sistema não perturbado. No capítulo 2 obtivemos a expressão (2.26) para $H_{\text {probe }}$ em termos dos operadores $\Phi_{0 \mu}(R)$ e $\phi_{\mu}(R)$ definidos em (2.24) e (2.25), respectivamente. Mostramos que a projeção de $H_{A}$ na base contínua $\left\{c_{\varepsilon}, \bar{c}_{\varepsilon}\right\}$ resulta no Hamiltoniano $\tilde{H}$ contendo o termo $\bar{H}_{c}$ de contribuição perturbativa. Assim, concluímos que os $\Phi_{0 \mu}(R)$ são dependentes exclusivamente dos operadores $\bar{c}_{\varepsilon}$ da base contínua e, uma vez que os autoestados do Hamiltoniano perturbado $\tilde{H}$ são desacoplados, podemos tratar cada termo $\phi_{\mu}(R)$ e $\Phi_{0 \mu}(R)$ independentemente.

Tendo definido as transformações (3.9) e (3.10), que relacionam os operadores fermiônicos $c_{\varepsilon}$ e $\bar{c}_{\varepsilon}$ à base do NRG $\left\{f_{n}\right\}$, vamos aproveitar o desacoplamento dos $\bar{c}_{\varepsilon}$ do sistema nãoperturbado, descrito por $H_{A}$, para reescrever $\phi_{\mu}(R)$ em uma forma numérica.

*Veja a seção 4.2.2. 


\subsection{Hamiltoniano da ponta de prova na base de Lanc- zos}

Considerando as contribuições independentes de (2.24) e (2.25), vamos expressar $H_{\text {probe }}$ em termos da base $\left\{f_{n}, \bar{c}_{\varepsilon}\right\}$. Para isso, vamos propor que o operador $\phi_{\mu}(R)$ pode ser escrito como

$$
\phi_{\mu}(R)=\sum_{n} \gamma_{n} f_{n \mu}
$$

onde os coeficientes $\gamma_{n}$ são determinados pelo comutador $\left\{f_{n \nu}^{\dagger}, \phi_{\mu}(R)\right\}$

$$
\gamma_{n}=\left\{f_{n \nu}^{\dagger}, \phi_{\mu}(R)\right\}=\sum_{m} u_{n m}\left(\zeta_{m}^{+}+(-1)^{n} \zeta_{m}^{-}\right)
$$

com

$$
\zeta_{m}^{+}=\left\{a_{m \nu}^{\dagger}, \phi_{\mu}(R)\right\}=\delta_{\mu, \nu} \frac{\Lambda^{m / 2}}{\sqrt{D\left(1-\Lambda^{-1}\right)}} \int_{\mathcal{I}_{m+}} d \varepsilon W(\varepsilon, R)
$$

e

$$
\zeta_{m}^{-}=\left\{b_{m \nu}^{\dagger}, \phi_{\mu}(R)\right\}=\delta_{\mu, \nu} \frac{\Lambda^{m / 2}}{\sqrt{D\left(1-\Lambda^{-1}\right)}} \int_{\mathcal{I}_{m-}} d \varepsilon W(\varepsilon, R),
$$

onde $W(\varepsilon, R)$ foi definido em (2.17).

A equação (4.3) relaciona os operadores $\phi_{\mu}(R)$ com os $f_{n}$ através dos coeficientes $\gamma_{n}$ calculados por (4.4). Tais coeficientes podem ser obtidos resolvendo as integrais (4.5) e (4.6) para uma distância $R$ fixa. Assim, usando a definição (2.17), devemos calcular

$$
\mathcal{S}_{m+}(R)=\mathcal{E}_{m} \int_{\mathcal{I}_{m+}} \frac{d \varepsilon}{\varepsilon} W(\varepsilon, R)=\int_{D \Lambda^{-m-1}}^{D \Lambda^{-m}} d \varepsilon \frac{\sin \left(k_{F} R\left(1+\frac{\varepsilon}{D}\right)\right)}{k_{F} R\left(1+\frac{\varepsilon}{D}\right)},
$$

e

$$
\mathcal{S}_{m-}(R)=\mathcal{E}_{m} \int_{\mathcal{I}_{m-}} \frac{d \varepsilon}{\varepsilon} W(\varepsilon, R)=\int_{-D \Lambda^{-m}}^{-D \Lambda^{-m-1}} d \varepsilon \frac{\sin \left(k_{F} R\left(1+\frac{\varepsilon}{D}\right)\right)}{k_{F} R\left(1+\frac{\varepsilon}{D}\right)} .
$$

Considerando conhecidos os valores das integrais $\mathcal{S}_{m \pm}$ (calculados por integração numérica ou pelas aproximações dos casos assintóticos) e todos os $u_{n m}$, podemos determinar os coeficientes $\gamma_{n}$ da transformação (4.3) através da relação

$$
\gamma_{n}(R)=\sum_{m}\left(\mathcal{S}_{m+}(R)+(-1)^{n} \mathcal{S}_{m-}(R)\right) \frac{u_{n m}}{u_{0 m}},
$$

onde substituímos os $v_{n m}$ por (3.20).

Emboras as integrais $\mathcal{S}_{m \pm}$ nas equações (4.7) e (4.8) possam ser efetuadas, a solução não é atraente. Como explicamos no capítulo 2 , não podemos aproximar o valor efetivo de $k$ por 
$k_{F}$, de modo que é mais prático efetuar as integrações numericamente.

Finalmente, tendo determinado todos os $\gamma_{n}$ através da expressão (4.9), somos capazes de reescrever o Hamiltoniano $H_{\text {probe }}$ na base $\left\{f_{n}, \bar{c}_{\varepsilon}\right\}$

$$
\begin{aligned}
H_{\text {probe }}= & -A\left[\iint_{-D}^{D} d \varepsilon d \varepsilon^{\prime} \sqrt{1-W(\varepsilon, R)} \sqrt{1-W\left(\varepsilon^{\prime}, R\right)} \bar{c}_{\varepsilon \mu}^{\dagger} \bar{c}_{\varepsilon^{\prime} \nu}\right. \\
& +\sum_{n} \gamma_{n}\left(\int_{-D}^{D} d \varepsilon \sqrt{1-W(\varepsilon, R)} \bar{c}_{\varepsilon \mu}^{\dagger} f_{n \nu}+\int_{-D}^{D} d \varepsilon \sqrt{1-W(\varepsilon, R)} f_{n \mu}^{\dagger} \bar{c}_{\varepsilon \nu}\right) \\
& \left.+\sum_{n, m} \gamma_{n} \gamma_{n^{\prime}} f_{n \mu}^{\dagger} f_{m \nu}\right] \mathbf{I}+\text { H.c. },
\end{aligned}
$$

$\operatorname{com} \mu=\uparrow$ e $\nu=\downarrow$.

A expressão (4.10) para $H_{\text {probe }}$ na base $\left\{\bar{c}_{\varepsilon}, f_{n}\right\}$ revela que a ponta de prova pode ser decomposta em três contribuições: o termo $\bar{c}_{\varepsilon \uparrow}^{\dagger} \bar{c}_{\varepsilon \downarrow}\left(\right.$ ou $\Phi_{0 \uparrow}^{\dagger} \Phi_{0 \downarrow}$ ) é associado somente aos estados de onda $s$ da ponta de prova ortogonais aos estados da impureza; a contribuição $f_{n \uparrow}^{\dagger} f_{m \downarrow}$ (ou $\left.\phi_{\uparrow}^{\dagger} \phi_{\downarrow}\right)$ abrange somente estados do sistema metálico com a impureza na base de Lanczos $\left\{f_{n}\right\}$; finalmente, elementos da forma $f_{n}^{\dagger} \bar{c}_{\varepsilon}$ e $\bar{c}_{\varepsilon}^{\dagger} f_{n}$ (ou $\phi_{\uparrow}^{\dagger} \Phi_{0 \downarrow}$ e $\Phi_{0 \uparrow}^{\dagger} \phi_{\downarrow}$ ) são combinações simultâneas dos estados da ponta de prova e da impureza, sendo responsável pela interferência entre ambos.

Na prática, para computar a taxa de relaxação $1 / T_{1}$ em (4.1) precisamos obter os elementos de matriz de $H_{\text {probe }}$ entre os autoestados de $\tilde{H}$, isto é,

$$
\begin{aligned}
\left\langle\tilde{\varphi}\left|H_{\text {probe }}\right| \tilde{\psi}\right\rangle \propto & \left\langle\tilde{\varphi}\left|\Phi_{0 \uparrow}^{\dagger} \Phi_{0 \downarrow}\right| \tilde{\psi}\right\rangle+\sum_{n} \gamma_{n}\left\langle\tilde{\varphi}\left|\left(\Phi_{0 \uparrow}^{\dagger} f_{n \downarrow}+f_{n \uparrow}^{\dagger} \Phi_{0 \downarrow}\right)\right| \tilde{\psi}\right\rangle \\
& +\sum_{n, m} \gamma_{n} \gamma_{m}\left\langle\tilde{\varphi}\left|f_{n \uparrow}^{\dagger} f_{m \downarrow}\right| \tilde{\psi}\right\rangle,
\end{aligned}
$$

onde $|\tilde{\varphi}\rangle$ e $|\tilde{\psi}\rangle$ têm energias $\tilde{E}_{\varphi}$ e $\tilde{E}_{\psi}$, respectivamente.

A partir da relação (4.11) inferimos que a taxa de relaxação depende somente do cálculo de elementos de matriz da forma $\left\langle\tilde{\varphi}\left|\Phi_{0 \uparrow}^{\dagger} \Phi_{0 \downarrow}\right| \tilde{\psi}\right\rangle,\left\langle\tilde{\varphi}\left|\Phi_{0 \mu}^{\dagger} \phi_{\nu}\right| \tilde{\psi}\right\rangle$, $\left\langle\tilde{\varphi}\left|\phi_{\mu}^{\dagger} \phi_{\nu}\right| \tilde{\psi}\right\rangle$ e seus Hermitianos conjugados. Em particular, a contribuição $\Phi_{0 \uparrow} \Phi_{0 \downarrow}$, que chamamos de contribuição escalar, corresponde a uma constante e, portanto, não será considerada no cálculo de $1 / T_{1}$.

Assim, vamos trabalhar somente com as componentes $\Phi_{0 \mu}^{\dagger} \phi_{\nu}$ e $\phi_{\mu}^{\dagger} \phi_{\nu}$, cujas respectivas contribuições serão denotadas vetorial e matricial. A justificativa para a adoção desta terminologia se encontra no tipo de dependência que cada contribuição tem com os estados acoplados 
à impureza: a componente $\Phi_{0 \mu}^{\dagger} f_{n \nu}$ depende de $N$ elementos de matriz da forma $\left\langle\varphi\left|f_{n \nu}\right| \psi\right\rangle$ e a componente $\phi_{\mu}^{\dagger} \phi_{\nu}$ depende de $N^{2}$ elementos. Os detalhes no cálculo das contribuições vetorial e matricial será apresentado a seguir nas subseções 4.2.1 e 4.2.2.

\subsubsection{Contribuição vetorial}

Voltando à expressão (2.29) derivada no capítulo 2 , recordamos que os $\bar{c}_{\varepsilon}$ desacoplados dos $f_{n}$, de modo que as contribuições dos termos contendo $\Phi_{0 \mu}$ podem ser tratadas analiticamente.

Substituindo as definições de $\Phi_{0 \mu}(R)$ e $\phi_{\mu}(R)$, respecitvamente em (2.25) e (4.3), podemos reescrever o terceiro termo à direita da equação (4.11) como

$$
\left\langle\tilde{\varphi}\left|\phi_{\mu}^{\dagger}(R) \Phi_{0 \nu}(R)\right| \tilde{\psi}\right\rangle=\sum_{n} \gamma_{n}\left\langle\tilde{\varphi}\left|f_{n \mu}^{\dagger} \int_{-D}^{D} d \varepsilon \sqrt{1-W(\varepsilon)^{2}} \bar{c}_{\varepsilon \nu}\right| \tilde{\psi}\right\rangle
$$

onde $|\tilde{\varphi}\rangle=|\bar{\varphi}\rangle|\varphi\rangle$ and $|\tilde{\psi}\rangle=|\bar{\psi}\rangle|\psi\rangle,|\varphi\rangle$ e $|\psi\rangle$ são autoestados de $H_{A}$ não-perturbado com energias $E_{\varphi}$ and $E_{\psi}$, respectivamente, e $\gamma_{n}$ é definido em (4.9). Segue que

$$
\left\langle\tilde{\varphi}\left|\phi_{\mu}^{\dagger}(R) \Phi_{0 \nu}(R)\right| \tilde{\psi}\right\rangle=\left\langle\varphi^{\prime}\left|\left\langle\bar{\varphi}\left|\phi_{\mu}^{\dagger}(R) \int_{-D}^{D} d \varepsilon \sqrt{1-W(\varepsilon)^{2}} \bar{c}_{\varepsilon \nu}\right| \bar{\psi}\right\rangle\right| \psi^{\prime}\right\rangle .
$$

Podemos decompor os elementos de matriz na equação (4.13), separando os $\left\langle\varphi\left|f_{n}^{\dagger}\right| \psi\right\rangle$ dos $\left\langle\bar{\varphi}\left|\bar{c}_{\varepsilon \nu}\right| \bar{\psi}\right\rangle$, ou seja,

$$
\left\langle\tilde{\varphi}\left|\phi_{\mu}^{\dagger}(R) \Phi_{0 \nu}(R)\right| \tilde{\psi}\right\rangle=\left\langle\varphi\left|\phi_{\mu}^{\dagger}(R)\right| \psi\right\rangle\left\langle\bar{\varphi}\left|\int_{-D}^{D} d \varepsilon \sqrt{1-W(\varepsilon)^{2}} \bar{c}_{\varepsilon \nu}\right| \bar{\psi}\right\rangle .
$$

O segundo termo à direita de (4.14) conecta os estados $|\bar{\varphi}\rangle$ e $|\bar{\psi}\rangle$ pela relação

$$
|\bar{\varphi}\rangle=\bar{c}_{\varepsilon \nu}|\bar{\psi}\rangle
$$

tal que o elemento de matriz existe somente se o estado $|\bar{\psi}\rangle$ não é vazio.

Assim, o elemento de matriz de $\bar{c}_{\varepsilon}$ entre os estados de $\bar{H}$ exige que as energias da transição de $|\bar{\varphi}\rangle$ e $|\bar{\psi}\rangle$ satisfaçam

$$
\left\langle\bar{\varphi}\left|\bar{c}_{\varepsilon \nu}\right| \bar{\psi}\right\rangle=\delta\left(E_{\bar{\varphi}}-E_{\bar{\psi}}-\varepsilon\right)
$$

seguindo que

$$
\left\langle\bar{\varphi}\left|\phi_{\mu}^{\dagger}(R) \Phi_{0 \nu}(R)\right| \bar{\psi}\right\rangle=\left\langle\varphi\left|\phi_{\mu}^{\dagger}(R)\right| \psi\right\rangle \sqrt{\left.1-W\left(E_{\varphi}-E_{\psi}\right)\right)^{2}} .
$$


Denotando $\left(1 / T_{1}\right)_{v e t}$ a taxa de relaxação magnética devida ao termo $\left\langle\bar{\varphi}\left|\phi_{\mu}^{\dagger}(R) \Phi_{0 \nu}(R)\right| \bar{\psi}\right\rangle$, obtemos

$$
\left(\frac{1}{T_{1}}\right)_{v e t}=\frac{4 \pi}{\hbar Z} \sum_{|\varphi\rangle,|\psi\rangle}\left|\left\langle\varphi\left|\phi_{\mu}^{\dagger}(R)\right| \psi\right\rangle\right|^{2}\left(1-W\left(E_{\varphi}-E_{\psi}\right)^{2}\right) e^{-\beta\left(E_{\varphi}\right)} f_{F D}\left(E_{\varphi}-E_{\psi}\right),
$$

onde $Z$ é a função de partição do sistema $H_{A}$ não-perturbado incluindo todos os estados $|\varphi\rangle$ e $|\psi\rangle$ e $f_{F D}(\varepsilon)$ é a distribuição de Fermi-Dirac definida como

$$
f_{F D}(\varepsilon)=\frac{1}{1+e^{-\beta \varepsilon}}
$$

Manipulando a equação (4.18) obtemos a seguinte expressão analítica para $\left(1 / T_{1}\right)_{\text {vet }}$

$$
\left(\frac{1}{T_{1}}\right)_{v e t}=\frac{4 \pi}{\hbar Z} \sum_{|\varphi\rangle,|\psi\rangle}\left(1-W\left(E_{\varphi}-E_{\psi}\right)^{2}\right) \frac{\left|\left\langle\varphi\left|\phi_{\mu}^{\dagger}(R)\right| \psi\right\rangle\right|^{2}}{e^{\beta E_{\varphi}}+e^{\beta E_{\psi}}}
$$

Podemos repetir os passos (4.12)-(4.20) para obter o termo $\left\langle\varphi\left|\Phi_{0 \mu}^{\dagger}(R) \phi_{\nu}(R)\right| \psi\right\rangle$. O resultado é equivalente a $\left(1 / T_{1}\right)_{\text {vet }}$ obtido em (4.20) trocando $|\varphi\rangle$ por $|\psi\rangle$, de modo que a taxa total com ambos os termos cruzados é obtida multiplicando esta expressão por dois.

De acordo com última expressao (4.20), o cálculo de $\left(1 / T_{1}\right)_{v e t}$, dependente de termos do tipo $\left\langle\varphi\left|\phi_{\nu}^{\dagger}(R) \Phi_{0 \mu}(R)\right| \psi\right\rangle$, pode ser efetuado através da computação dos elementos de matriz $\left\langle\varphi\left|f_{n \mu}^{\dagger}\right| \psi\right\rangle$ entre os estados do Hamiltoniano $H_{A}$ não perturbado. Esse procedimento é relativamente simples, uma vez que os $\left\langle\varphi\left|f_{n \mu}^{\dagger}\right| \psi\right\rangle$ estão associados aos elementos de matriz reduzidos $\left\langle\varphi\left\|f_{n}^{\dagger}\right\| \psi\right\rangle$ pelo teorema de Wigner-Eckart. Vimos na seção 3.6 .4 que todos os $N$ invariantes de uma dada iteração podem ser obtidos recursivamente a partir dos elementos $\left\langle\varphi|| f_{N}^{\dagger}|| \psi\right\rangle$, os quais são um subproduto do procedimento iterativo.

A próxima seção é dedicada ao cálculo da contribuição do termo $\left\langle\tilde{\varphi}\left|f_{n \mu}^{\dagger} f_{m \nu}\right| \tilde{\psi}\right\rangle$ à taxa $1 / T_{1}$. Apresentaremos diferentes formas de realizar este cálculo, mostrando suas vantagens e desvantagens no contexto do Grupo de Renormalização Numérico.

\subsubsection{Contribuição matricial}

Para calcular a contribuição matricial dos operadores $\phi_{\uparrow}^{\dagger}(R) \phi_{\downarrow}(R)$ devemos encontrar os elementos de matriz dos autoestados de $\tilde{H}$. Vimos no capítulo 2 e na seção 4.2 .1 que os estados $\bar{H}_{c}$ e $H_{A}$ são desacoplados, de forma que a soma de estados que contribuem para a 
taxa $\left(1 / T_{1}\right)_{\text {mat }}$ associada ao termo matricial é dada por

$$
\left(\frac{1}{T_{1}}\right)_{m a t}=\frac{4 \pi}{\hbar Z} \sum_{|\varphi\rangle,|\psi\rangle} e^{-\beta E_{\varphi}}\left|\left\langle\varphi\left|\phi_{\uparrow}^{\dagger}(R) \phi_{\downarrow}(R)\right| \psi\right\rangle\right|^{2} \delta\left(E_{\varphi}-E_{\psi}\right),
$$

onde $|\varphi\rangle$ e $|\psi\rangle$ são os autoestados do Hamiltoniano de Anderson $H_{A}$.

Rigorosamente, a expressão (4.1) é escrita como

$$
\left(\frac{1}{T_{1}}\right)_{m a t}=\frac{4 \pi}{\hbar Z} \sum_{|\varphi\rangle,|\psi\rangle} e^{-\beta E_{\varphi}}\left|\left\langle\varphi\left|\phi_{\uparrow}^{\dagger}(R) \phi_{\downarrow}(R)\right| \psi\right\rangle\right|^{2} \delta\left(E_{\varphi}-E_{\psi}-\hbar \omega\right),
$$

de modo que a transição acontece entre os estados $|\varphi\rangle$ e $|\psi\rangle$ que diferem energeticamente pela quantidade $\hbar \omega$.

Na prática, como $|\varphi\rangle$ e $|\psi\rangle$ são estados da banda de condução com energias inicialmente diferentes $E_{\varphi}>E_{\psi}, H_{\text {probe }}(\omega)$ inverte seus spins e desloca a energia $E_{\varphi}$ infinitesimalmente. Analiticamente, a diferença energética final entre esses estados pode ser computada como

$$
\left(\frac{1}{T_{1}}\right)_{m a t}=\frac{4 \pi}{\hbar Z} \sum_{|\varphi\rangle,|\psi\rangle} e^{-\beta E_{\varphi}} \frac{\left|\left\langle\varphi\left|\phi_{\uparrow}^{\dagger} \phi_{\downarrow}\right| \psi\right\rangle\right|^{2}}{\left|\frac{d\left(E_{\varphi}(z)\right)-E_{\psi}(z)}{d z}\right|_{\hbar \omega=E_{\psi}-E_{\varphi}}},
$$

onde as energias $E_{\varphi}(z)$ e $E_{\psi}(z)$ são os autovalores de $H_{A}$ no formalismo do Grupo de Renormalização Numérico generalizado (46).

A derivada no denominador da soma à direita da equação (4.23) é exatamente a densidade de estados $\rho$. Desse modo, o cálculo de $1 /\left(T_{1}\right)^{\text {mat }}$ depende essencialmente dos elementos de matriz $\left\langle\varphi\left|f_{n \mu}^{\dagger} f_{m \nu}\right| \psi\right\rangle$ entre os estados do Hamiltoniano $H_{A}$ não-perturbado. Nossa tarefa consiste, pois, em calcular

$$
\left\langle\tilde{\varphi}\left|\phi_{\mu}^{\dagger}(R) \phi_{\nu}(R)\right| \tilde{\psi}\right\rangle=\langle\bar{\varphi} \mid \bar{\psi}\rangle \sum_{n, m} \gamma_{n} \gamma_{m}\left\langle\varphi\left|f_{n \mu}^{\dagger} f_{m \nu}\right| \psi\right\rangle
$$

com $\nu=-\mu=(\uparrow, \downarrow)$ e $(n, m=0, \ldots, N)$.

Elementos deste tipo podem ser obtidos de duas formas. A primeira diz respeito à completeza dos estados $f_{n \mu}|\varphi\rangle$ e $f_{m \nu}|\psi\rangle$, enquanto a outra consiste em determinar relações recorrência envolvendo os elementos $\left\langle\varphi\left\|f_{n}^{\dagger} f_{m}\right\| \psi\right\rangle$ para $n, m=0, \ldots N$.

Em nosso trabalho, não iremos utilizar a completeza para o cálculo dos elementos matriciais: embora a completeza seja uma identidade, há erros numéricos envolvendo o truncamento dos estados cujo efeito é mais dramático nas últimas iterações. No apêndice $A$ apresentamos uma tentativa de implementar a completeza para o cálculo dos $\left\langle\varphi\left|f_{n \mu}^{\dagger} f_{m \nu}\right| \psi\right\rangle$, discutindo os 
problemas práticos envolvendo seu uso.

A segunda alternativa que propomos é mais promissora do ponto de vista prático, porque nos leva a uma expressão análoga à relação recursiva (3.83) obtida no capítulo 3 para determinar recursivamente o conjunto de invariantes $\left\langle\varphi|| f_{n}^{\dagger} \| \psi\right\rangle(\operatorname{com} n=0, \ldots, N)$. Uma vez que a implementação da recorrência (3.83) apresenta resultados numéricos satisfatórios (veja o apêndice C), acreditamos que esta maneira de computar os $\left\langle\varphi\left|f_{n \mu}^{\dagger} f_{m \nu}\right| \psi\right\rangle$ poderia ser eficiente e mais precisa.

Para obter as relações de recorrência entre os $\left\langle\varphi\left|f_{n \mu}^{\dagger} f_{m \nu}\right| \psi\right\rangle$ para todos os inteiros $n, m=$ $0, \ldots, N$, começamos calculando os elementos de matriz dos estados $|\varphi\rangle$ e $|\psi\rangle$ com o comutador

$$
\left[H, f_{n \uparrow}^{\dagger} f_{m \downarrow}\right]=\left[H, f_{n \uparrow}^{\dagger}\right] f_{m \downarrow}+f_{n \uparrow}^{\dagger}\left[H, f_{m \downarrow}\right]
$$

Usando a relação (3.77), calculamos ambos os comutadores à direita da equação (4.25)

$$
\left[H, f_{n \uparrow}^{\dagger}\right] f_{m \downarrow}=t_{n-1} f_{n-1 \uparrow}^{\dagger} f_{m \downarrow}+t_{n} f_{n+1 \uparrow}^{\dagger} f_{m \downarrow}
$$

e

$$
f_{n \uparrow}^{\dagger}\left[H, f_{m \downarrow}\right]=t_{m-1} f_{n \uparrow}^{\dagger} f_{m-1 \downarrow}+t_{m} f_{n \uparrow}^{\dagger} f_{m+1 \downarrow},
$$

bem como seus respectivos elementos de matriz reduzidos

$$
\begin{aligned}
t_{n-1}\left\langle\varphi\left\|f_{n-1}^{\dagger} f_{m}\right\| \psi\right\rangle+t_{n}\left\langle\varphi\left\|f_{n+1}^{\dagger} f_{m}\right\| \psi\right\rangle & =\left\langle\varphi\left\|\left[H, f_{n}^{\dagger}\right] f_{m}\right\| \psi\right\rangle \\
& =E_{\varphi}\left\langle\varphi\left\|f_{n}^{\dagger} f_{m}\right\| \psi\right\rangle-\left\langle\varphi\left\|f_{n}^{\dagger} H f_{m}\right\| \psi\right\rangle,
\end{aligned}
$$

e

$$
\begin{aligned}
t_{m-1}\left\langle\varphi\left\|f_{n}^{\dagger} f_{m-1}\right\| \psi\right\rangle+t_{m}\left\langle\varphi\left\|f_{n}^{\dagger} f_{m+1}\right\| \psi\right\rangle & =\left\langle\varphi\left\|f_{n}^{\dagger}\left[H, f_{m}\right]\right\| \psi\right\rangle \\
& =\left\langle\varphi\left\|f_{n}^{\dagger} H f_{m}\right\| \psi\right\rangle-E_{\psi}\left\langle\varphi\left\|f_{n}^{\dagger} f_{m}\right\| \psi\right\rangle
\end{aligned}
$$

Substituindo as relações (4.28) e (4.29) em (4.25), obtemos

$$
\begin{aligned}
\left(E_{\varphi}-E_{\psi}\right)\left\langle\varphi\left\|f_{n}^{\dagger} f_{m}\right\| \psi\right\rangle & =t_{n-1}\left\langle\varphi\left\|f_{n-1}^{\dagger} f_{m}\right\| \psi\right\rangle+t_{n}\left\langle\varphi\left\|f_{n+1}^{\dagger} f_{m}\right\| \psi\right\rangle \\
& -t_{m-1}\left\langle\varphi\left\|f_{n}^{\dagger} f_{m-1}\right\| \psi\right\rangle-t_{m}\left\langle\varphi\left\|f_{n}^{\dagger} f_{m+1}\right\| \psi\right\rangle .
\end{aligned}
$$

A equação (4.30) é semelhante à relação de recorrência (3.83) para os $f_{n}^{\dagger}$, exigindo um pouco mais de cuidado por envolver dois índices. Em princípio, seria conveniente representar a recorrência (4.30) através de uma matriz de elementos reduzidos $\left\langle\varphi \mid\left\|f_{n}^{\dagger} f_{m}\right\| \psi\right\rangle$ com $N+1$ linhas e $N+1$ colunas, tal como ilustramos na figura 4.1. Assim, para cada par de estados $|\varphi\rangle=$ 
$\left|q^{\prime}, s^{\prime}, r^{\prime}\right\rangle$ e $|\psi\rangle=|q, s, r\rangle$ temos de computar uma matriz de invariantes $\left\langle q^{\prime}, s^{\prime}, r^{\prime}\left\|f_{n}^{\dagger} f_{m}\right\| q, s, r\right\rangle$ $(n, m=0, \ldots, N)$ cujos elementos são utilizados no cálculo da taxa $1 /\left(T_{1}\right)^{\text {mat }}$.

Obviamente, como os operadores $f_{n}\left(f_{n}^{\dagger}\right)$ aniquilam (criam) elétrons em um dado estado e, por conseguinte, modificam sua carga e seu spin, precisamos identificar quais estados $|q, s, r\rangle$ e $\left|q^{\prime}, s^{\prime}, r^{\prime}\right\rangle$ contribuem efetivamente para a relaxação, ou seja, devemos determinar as regras de seleção para a carga e o spin desses estados quando conectados pelo operador $f_{n}^{\dagger} f_{m}$ $(n, m=0, \ldots, N)$. Para um ket $|q, s, r\rangle$ fixo, computamos apenas as matrizes de invariantes $\left\langle q^{\prime}, s^{\prime}, r^{\prime}\left\|f_{n}^{\dagger} f_{m}\right\| q, s, r\right\rangle$ diagonais, tais que $\left|q^{\prime}, s^{\prime}, r^{\prime}\right\rangle$ e $|q, s, r\rangle$ têm $q^{\prime}=q$ e $s^{\prime}=s$, com componentes $s_{z}$ que podem diferir entre si.

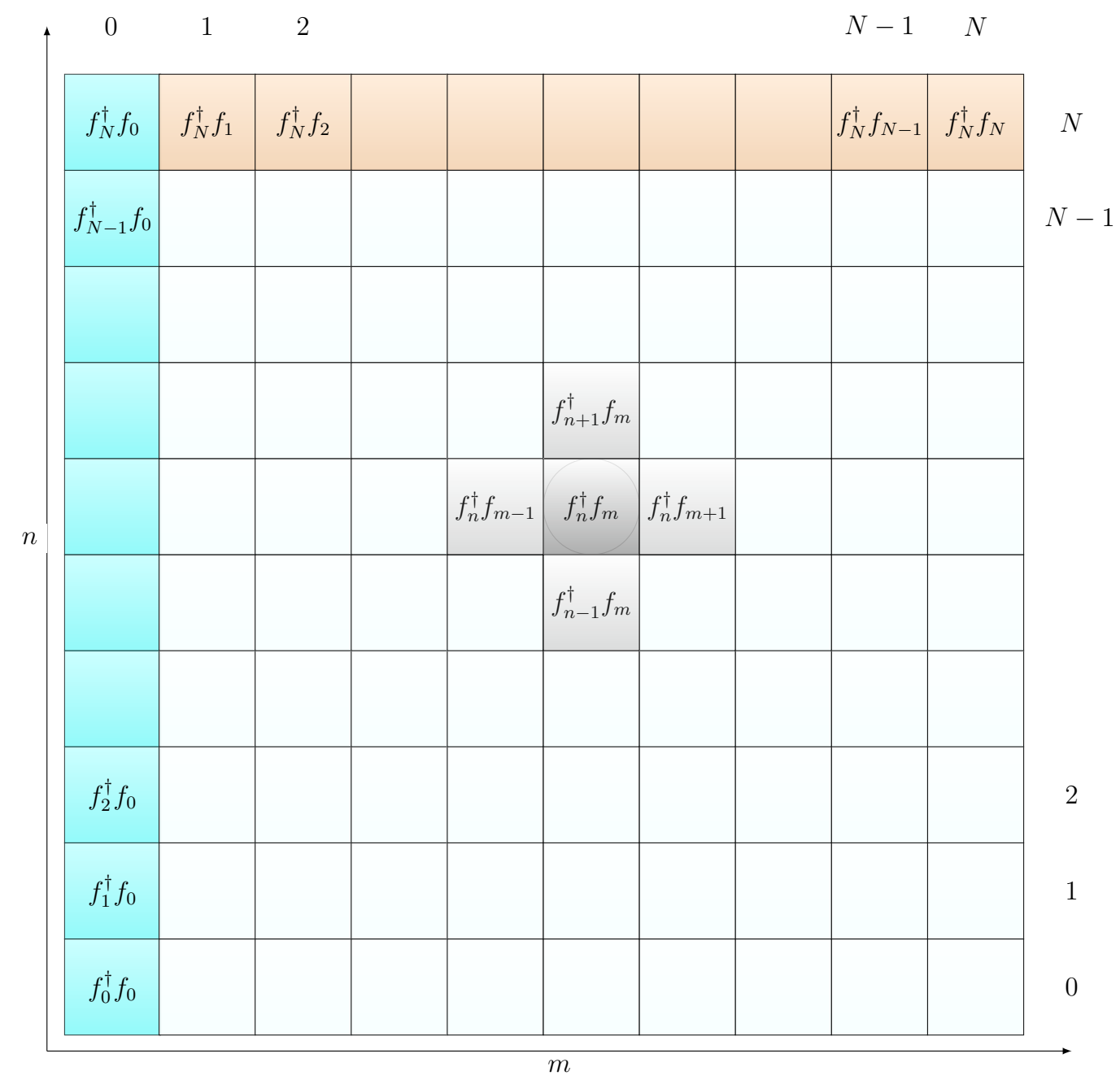

Figura 4.1 - Disposição dos elementos invariantes $\left\langle\varphi\left\|f_{n}^{\dagger} f_{m}\right\| \psi\right\rangle$ em uma matriz de $n$ linhas e $m$ colunas, com $m, n=0, \ldots, N$. Cada elemento $\left\langle\varphi\left\|f_{n}^{\dagger} f_{m}\right\| \psi\right\rangle$ pode ser determinado pelos vizinhos $\left\langle\varphi\left\|f_{n \pm 1}^{\dagger} f_{m \pm 1}\right\| \psi\right\rangle$. Fonte: elaborada pela autora.

Tendo definido os estados $|q, s, r\rangle$ e $\left|q^{\prime}, s^{\prime}, r^{\prime}\right\rangle$ que contribuem para a taxa $1 /\left(T_{1}\right)^{\text {mat }}$, podemos prosseguir com o cálculo dos conjuntos de $N^{2}$ invariantes representados pela matriz 
da figura 4.1. Por ora, sabemos que um dado elemento $\left\langle\varphi\left\|f_{n}^{\dagger} f_{m}\right\| \psi\right\rangle$ pode ser determinado através da relação de recorrência (4.30), o que exige conhecimento prévio dos seus vizinhos $\left\langle\varphi|| f_{n-1}^{\dagger} f_{m}|| \psi\right\rangle,\left\langle\varphi|| f_{n}^{\dagger} f_{m+1}|| \psi\right\rangle,\left\langle\varphi\left\|f_{n+1}^{\dagger} f_{m}\right\| \psi\right\rangle$ e $\left\langle\varphi\left\|f_{n}^{\dagger} f_{m-1}\right\| \psi\right\rangle$. Observando que nas bordas da matriz a relação (4.30) é mais simples, devemos ser capazes de encontrar uma forma eficiente de determinar todo o conjunto de $N^{2}$ invariantes entre estados $|\psi\rangle$ e $|\varphi\rangle$.

Reescrevendo a relação (4.30) para um elemento da $N$-ésima linha, segue que

$$
\begin{aligned}
\left(E_{\varphi}-E_{\psi}\right)\left\langle\varphi\left\|f_{N}^{\dagger} f_{m}\right\| \psi\right\rangle= & t_{N-1}\left\langle\varphi\left\|f_{N-1}^{\dagger} f_{m}\right\| \psi\right\rangle-t_{m-1}\left\langle\varphi\left\|f_{N}^{\dagger} f_{m-1}\right\| \psi\right\rangle \\
& -t_{m}\left\langle\varphi\left\|f_{N}^{\dagger} f_{m+1}\right\| \psi\right\rangle,
\end{aligned}
$$

ou, invertendo a relação para destacar o elemento $\left\langle\varphi|| f_{N-1}^{\dagger} f_{m} \| \psi\right\rangle$,

$$
\begin{aligned}
\left\langle\varphi\left\|f_{N-1}^{\dagger} f_{m}\right\| \psi\right\rangle= & \frac{t_{m-1}}{t_{N-1}}\left\langle\varphi\left\|f_{N}^{\dagger} f_{m-1}\right\| \psi\right\rangle+\frac{t_{m}}{t_{N-1}}\left\langle\varphi\left\|f_{N}^{\dagger} f_{m+1}\right\| \psi\right\rangle \\
& +\frac{\left(E_{\varphi}-E_{\psi}\right)}{t_{N-1}}\left\langle\varphi\left\|f_{N}^{\dagger} f_{m}\right\| \psi\right\rangle .
\end{aligned}
$$

A equação (4.32) nos revela que conhecendo o conjunto de elementos de matriz reduzidos da linha $N$, é possível determinar os invariantes da linha $N-1$. Na prática, podemos reescrever a relação (4.31) para quaisquer elemento da borda da matriz de invariantes da figura 4.1, ou seja, para elementos do tipo $\left\langle\varphi\left\|f_{n}^{\dagger} f_{N}\right\| \psi\right\rangle,\left\langle\varphi\left\|f_{n}^{\dagger} f_{0}\right\| \psi\right\rangle,\left\langle\varphi\left\|f_{0}^{\dagger} f_{n}\right\| \psi\right\rangle$ ou $\left\langle\varphi\left\|f_{N}^{\dagger} f_{n}\right\| \psi\right\rangle$ $(n=0, \ldots, N)$. A relação obtida será análoga a (4.32) e permitirá determinar os elementos das linhas e colunas adjacentes indexadas pelos índices $N-1$ e 1 . Como a matriz dos invariantes $\left\langle\varphi|| f_{n}^{\dagger} f_{m} \| \psi\right\rangle$ é simétrica, determinar a linha $\left\langle\varphi\left\|f_{n}^{\dagger} f_{N}\right\| \psi\right\rangle$ é equivalente a determinar a coluna $\left\langle\varphi\left\|f_{N}^{\dagger} f_{n}\right\| \psi\right\rangle$, valendo o mesmo para $\left\langle\varphi\left\|f_{n}^{\dagger} f_{0}\right\| \psi\right\rangle$ e $\left\langle\varphi\left\|f_{0}^{\dagger} f_{n}\right\| \psi\right\rangle$. Conhecendo agora o conjunto de elementos da linha $N-1$, podemos reescrever a relação de recorrência (4.30) para todos os $\left\langle\varphi\left\|f_{N-1}^{\dagger} f_{m}\right\| \psi\right\rangle$, o que nos possibilita determinar toda a linha $N-2$. Prosseguindo até $N=0$ a matriz de invariantes pode ser facilmente computada.

Em particular, para o caso em que os estados $|q, s, r\rangle$ e $\left|q^{\prime}, s^{\prime}, r^{\prime}\right\rangle$ coincidem, a diferença de energias é nula. As relações para os invariantes das linhas $N-1$ e $N-2$ tornam-se

$$
\begin{aligned}
\left\langle q, d s, r^{\prime}\left\|f_{m}^{\dagger} f_{N-1}\right\| q, d s, r\right\rangle= & \frac{t_{m}}{t_{N-1}}\left\langle q, d s, r^{\prime}\left\|f_{m+1}^{\dagger} f_{N}\right\| q, d s, r\right\rangle \\
& +\frac{t_{m-1}}{t_{N-1}}\left\langle q, d s, r^{\prime}\left\|f_{m-1}^{\dagger} f_{N}\right\| q, d s, r\right\rangle
\end{aligned}
$$




$$
\begin{aligned}
\left\langle q, d s, r^{\prime}\left\|f_{m}^{\dagger} f_{n-1}\right\| q, d s, r\right\rangle= & \frac{t_{m}}{t_{n-1}}\left\langle q, d s, r^{\prime}\left\|f_{m+1}^{\dagger} f_{n}\right\| q, d s, r\right\rangle \\
& +\frac{t_{m-1}}{t_{n-1}}\left\langle q, d s, r^{\prime}\left\|f m-1^{\dagger} f_{n}\right\| q, d s, r\right\rangle \\
& -\frac{t_{n}}{t_{n-1}}\left\langle q, d s, r^{\prime}\left\|f_{m}^{\dagger} f_{n+1}\right\| q, d s, r\right\rangle,
\end{aligned}
$$

respectivamente, o que é ilustrado na figura 4.2.

As equações (4.33) determinam, respectivamente, os elementos ao longo das linhas $N-1$ e $N-2$ quando um estado $|q, s, r\rangle$ faz transições via $f_{n}$ e retorna ao mesmo estado.

A implementação do cálculo recursivo dos elementos dados na equação (4.30) é relativamente simples e eficiente, desde que tenhamos conhecimento prévio de todos os elementos da linha $n=N$ e da coluna $m=0$. Por outro lado, a computação desses últimos requer um método robusto, já que a completeza não pode ser usada no contexto numérico. A proposta que apresentamos no apêndice B mostra como calcular os elementos $\left\langle\varphi\left\|f_{N}^{\dagger} f_{m}\right\| \psi\right\rangle(m=0, \ldots, N)$ e $\left\langle\varphi\left\|f_{n}^{\dagger} f_{0}\right\| \psi\right\rangle(n=0, \ldots, N-1)$ através de rotações. Esse procedimento é análogo ao desenvolvido na seção 3.6.3 onde mostramos o cálculo dos invariantes, mas exige que outros elementos de matriz sejam calculados além dos diagonais.

A ideia básica da rotação consiste em reescrever os elementos de matriz da iteração $N$ na base de estados da iteração $N-1$ e através do teorema de Wigner-Eckart identificar os invariantes requeridos pela rotação. Para tanto, começamos reescrevendo os autoestados $\left|q, s, s_{z}, r\right\rangle$ e $\left|q^{\prime}, s^{\prime}, s_{z}^{\prime}, r^{\prime}\right\rangle$ na base primitiva

$$
\begin{aligned}
\left\langle q^{\prime}, s^{\prime}, s_{z}^{\prime}, r^{\prime}\left|f_{m \uparrow}^{\dagger} f_{m \downarrow}\right| q, s, s_{z}, r\right\rangle= & \sum_{p, p^{\prime}} U\left(p^{\prime}, r^{\prime}\right) U(p, r) \\
& \times\left\langle Q^{\prime}, S^{\prime}, S_{z}^{\prime}, p^{\prime}\left|\mathcal{O}_{g_{p^{\prime}}} f_{m \uparrow}^{\dagger} f_{m \downarrow} \mathcal{O}_{g_{p}}\right| Q, S, S_{z}, p\right\rangle,
\end{aligned}
$$

onde $U$ é a matriz da transformação unitária que diagonaliza $H_{N} ; \mathcal{O}_{g}$ é um dos operadores definidos pelas equações (3.57)-(3.60); $\left|Q, S, S_{z}, p\right\rangle$ e $\left|Q^{\prime}, S^{\prime}, S_{z}^{\prime}, p^{\prime}\right\rangle$ são os estados da iteração $N-1$ que deram origem aos estados $\left|q, s, s_{z}, r\right\rangle$ e $\left|q^{\prime}, s^{\prime}, s_{z}^{\prime}, r^{\prime}\right\rangle$ na iteração $N$.

Em seguida, usamos o teorema de Wigner-Eckart para relacionar os elementos de matriz $\left\langle q^{\prime}, s^{\prime}, s_{z}^{\prime}, r^{\prime}\left|f_{m \uparrow}^{\dagger} f_{m \downarrow}\right| q, s, s_{z}, r\right\rangle$ aos invariantes para obtermos a expressão

$$
\begin{aligned}
\left\langle q^{\prime}, s^{\prime}, s_{z}^{\prime}, r^{\prime}\left|f_{n \uparrow}^{\dagger} f_{m \downarrow}\right| q, s, s_{z}, r\right\rangle= & \left(\begin{array}{c|cc}
s^{\prime} & \frac{1}{2} & s+\frac{1}{2} \\
s_{z}^{\prime} & \frac{1}{2} & s_{z}-\frac{1}{2}
\end{array}\right)\left(\begin{array}{c|cc}
s & \frac{1}{2} & s \pm \frac{1}{2} \\
s_{z} & -\frac{1}{2} & s_{z}+\frac{1}{2}
\end{array}\right) \\
& \times\left\langle q^{\prime}, s^{\prime}, r^{\prime}\left\|f_{n}^{\dagger} f_{m}\right\| q, s, r\right\rangle .
\end{aligned}
$$




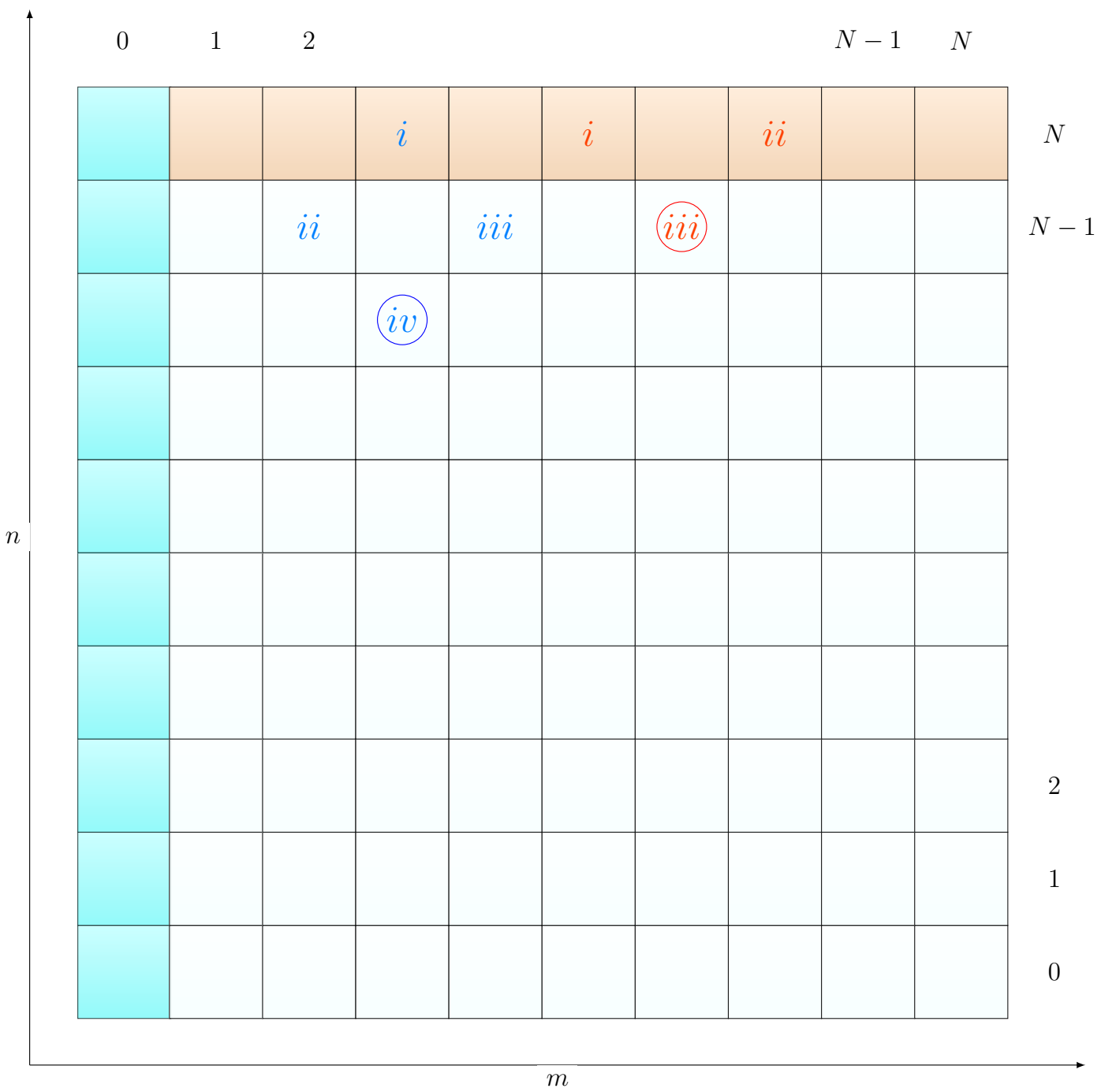

quando $|\varphi\rangle=|\psi\rangle]$

Figura 4.2 - Cálculo recursivo dos elementos de matriz reduzidos $\left\langle\varphi|| f_{n}^{\dagger} f_{m} \| \psi\right\rangle$ quando $|\varphi\rangle=|\psi\rangle$. No início da computação recursiva as linhas e colunas coloridas são conhecidas: a linha colorida em vermelho corresponde aos elementos de matriz da forma $\left\langle\varphi|| f_{N}^{\dagger} f_{m} \| \psi\right\rangle$ $(m=0, \ldots, N)$ e a coluna mostrada em azul diz respeito aos invariantes $\left\langle\varphi|| f_{n}^{\dagger} f_{0} \| \psi\right\rangle$ $(n=0, \ldots, N)$. Para obter um invariante $i i i$ da linha $N-1$ (circulado em vermelho) precisamos conhecer seus vizinhos $i$ ao noroeste e $i i$ ao nordeste, ambos localizados na linha $N$, aplicando a equação (4.33). Analogamente, para determinar um elemento $i v$ na linha $N-2$ (circulado em azul) devemos ter além de seus vizinhos noroeste ii e nordeste iii da linha $N-1$ o invariante $i$ da linha $N$. Com a equação (4.34) é possível determinar todos os elementos da mesma linha. Fonte: elaborada pela autora.

A soma à direita da equação (4.35) deve ser calculada para todos os pares de gêneros $g_{p^{\prime}}, g_{p}=\mathcal{N}, \mathcal{E}, \mathcal{S}, \mathcal{W}$, de modo a identificar o invariante à direita da equação (4.36) no elemento de matriz resultante, escrito como

$$
\left\langle q^{\prime}, s^{\prime}, r^{\prime}\left\|f_{n}^{\dagger} f_{m}\right\| q, s, r\right\rangle=\sum_{p, p^{\prime}} U(r, p) U\left(r^{\prime}, p^{\prime}\right) \mathcal{G}_{g_{p^{\prime}}, g_{p}}\left\langle Q^{\prime}, S^{\prime}, p^{\prime}\left\|f_{n}^{\dagger} f_{m}\right\| Q, S, p\right\rangle
$$


onde a matriz $\mathcal{G}$ abrange coeficientes dependentes dos gêneros $g_{p^{\prime}}$ e $g_{p}$. Os demais casos são discutidos no apêndice $B$.

Em síntese, este capítulo abordou o tratamento analítico do Hamiltoniano da ponta de prova $H_{\text {probe }}$ na base de Lanczos e o cálculo da taxa de relaxação magnética longitudinal $1 / T_{1}$. Conseguimos mostrar que o operador $\phi_{\mu}(R)$ pode ser definido como combinação linear dos $f_{n \mu}$ com coeficientes $\gamma_{n}$ relacionados à distância $R$ entre a impureza e ponta de prova e à energia. Com isto, pudemos obter a expressão (4.10) para $H_{\text {probe }}$ na base $\left\{\bar{c}_{\varepsilon}, f_{n}\right\}$, cuja projeção nos estados $\langle\varphi|$ e $|\psi\rangle$ do Hamiltoniano nos levou a identificar três contribuições para relaxação.

A taxa associada à contribuição escalar, dependente somente dos estados desacoplados da impureza, foi atribuída a uma constante de fundo que não afeta a relaxação total do sistema. Por outro lado, o termo dependente dos estados da impureza e da ponta de prova, denominado contribuição vetorial, foi calculado analiticamente na expressão (4.20). Mostramos que a taxa $\left(1 / T_{1}\right)_{\text {vet }}$ associada a esta contribuição depende basicamente dos invariantes $\left\langle\varphi\left\|f_{n}^{\dagger}\right\| \psi\right\rangle$ modulados por uma função de $R$ e das energias $E_{\varphi}$ e $E_{\psi}$. A contribuição matricial foi associada somente aos estados da impureza. Com a relação (4.21) demonstramos que a taxa $1 /\left(T_{1}\right)_{\text {mat }}$ requer o cálculo de elementos de matriz da forma $\left\langle\varphi\left|f_{n \uparrow}^{\dagger} f_{m \downarrow}\right| \psi\right\rangle$. Mostramos que a computação destes poderia ser feita implementando a recorrência (4.30), desde que conhecido o conjunto de elementos $\left\langle\varphi\left|f_{n \uparrow}^{\dagger} f_{0 \downarrow}\right| \psi\right\rangle(n=0, \ldots, N)$ e $\left\langle\varphi\left|f_{N \uparrow}^{\dagger} f_{m \downarrow}\right| \psi\right\rangle(m=0, \ldots, N)$.

Finalmente, podemos prosseguir para a abordagem numérica deste trabalho. No próximo capítulo dedicaremos especial atenção aos resultados numéricos da contribuição vetorial $\left(1 / T_{1}\right)_{v e t}$, discutindo seu comportamento com função $T$ e $k_{F} R$ e dando uma interpretação física da fenomenologia envolvida nesta taxa. 


\title{
Resultados
}

\author{
There are two possible outcomes: if the \\ result confirms the hypothesis, then \\ you've made a measurement. If the \\ result is contrary to the hypothesis, then \\ you've made a discovery. \\ Enrico Fermi
}

A seguir, apresentaremos os resultados numéricos da taxa de relaxação associada à contribuição vetorial. Antes, porém, de iniciarmos esta discussão, vamos fazer uma breve análise do comportamento dos coeficientes $\gamma_{n}$ definidos para a transformação do operador $\phi_{\mu}(R)$. Em particular, faremos um estudo do comportamento assintótico das integrais $\mathcal{S}_{m \pm}$ discutindo a validade das expansões e seu efeito sobre a taxa de relaxação.

\subsection{Coeficientes da transformação de Grupo de Re- normalização}

No capítulo 4 vimos que é possível escrever o Hamiltoniano $H_{\text {probe }}$ na base $\left\{f_{n}\right\}$ do NRG definindo a transformação (4.3) para o operador $\phi_{\mu}(R)$. Com isto, a atuação do operador $\phi_{\mu}(R)$ sobre os autoestados de $H_{A}$ torna-se uma tarefa simples, pois depende de elementos de matriz (análogos aos invariantes) multiplicados por amplitudes proporcionais às integrais $\mathcal{S}_{m \pm}$ multiplicadas pelos coeficientes da transformação unitária $u_{n m}$ e $v_{n m}$ da definição (3.13).

Os coeficientes $u_{n m}$ podem ser computados pela relação de recorrência (3.29) ou ainda através da função geradora

$$
U_{n}(z)=\sum_{m=0}^{\infty} u_{n m} z^{m} .
$$



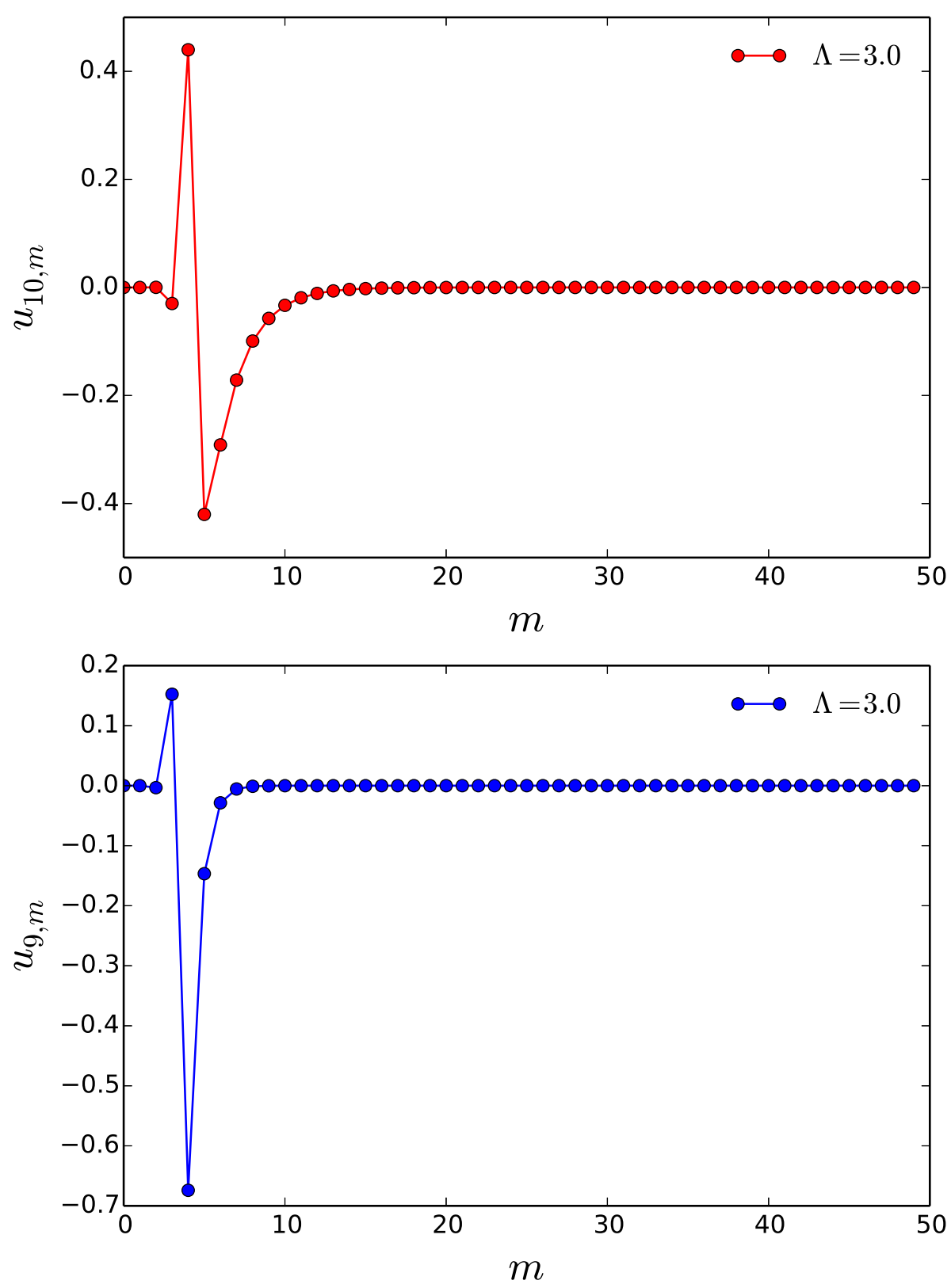

Figura 5.1 - Coeficientes $u_{10, m}$ e $u_{9, m}$ para $\Lambda=3.0$. O valor absoluto é máximo quando $m=n / 2$. Para $m>n / 2$, os coeficientes decaem à taxa $\Lambda^{(n / 2-m) / 2}$. O valor máximo de $n$ é definido pelos parâmetros do modelo, $E_{\min }$ e $\Lambda$, enquanto $m$ é arbitrariamente grande. O mesmo gráfico para $n>4$ revela que os $u_{n m}$ dependem somente da diferença $n / 2-m$. Fonte: elaborada pela autora.

Podemos observar o comportamento assintótico da função geradora $U_{n}(z)$. Para $n$ par, $u_{n m}$ decai à taxa $\Lambda^{-m / 2}$. Para $n$ ímpar, o decaimento é proporcional a $\Lambda^{-3 m / 2}$. Essa análise pode ser feita explicitamente, decompondo a função geradora $U_{n}(z)$ em funções polinomiais 
pares e ímpares, o que é feito através da relação (10)

$$
U_{n}(z)=\left(\frac{1-\Lambda^{-(2 n+1)}}{2 \Lambda^{n(n-1) / 2}}\right)^{1 / 2}\left(\frac{1}{1-z \Lambda^{-(n+1 / 2)}}\right) \times \begin{cases}\prod_{r=0}^{(n-2) / 2} \frac{1-z \Lambda^{2 r+1 / 2}}{1-z \Lambda^{-(2 r+1 / 2)}}, & n \text { par } \\ \prod_{r=0}^{(n-3) / 2} \frac{1-z \Lambda^{2 r+3 / 2}}{1-z \Lambda^{-(2 r+3 / 2)}}, & n \text { ímpar. }\end{cases}
$$

Em particular, para $n=0$ temos

$$
U_{0}(z)=\sqrt{\frac{1-\Lambda^{-1}}{2}}\left(\frac{1}{1-z \Lambda^{-(n+1 / 2)}}\right),
$$

seguindo que

$$
u_{0 m}=\frac{\alpha_{m}}{\sqrt{2}}
$$

Ainda no capítulo 4 mostramos que os coeficientes $\gamma_{n}$ envolvidos nesta transformação são escritos na forma geral através da equação (4.9). Com isto, o conjunto de $\gamma_{n}(n=0, \ldots, N)$ requer o cálculo dos coeficientes $u_{n m}$, abordados anteriormente, e das integrais $\mathcal{S}_{m \pm}$, que devem ser computadas numericamente dado que estas não têm solução analítica.

A partir dos valores de $\gamma_{n}$ obtidos a partir da integração numérica das somas $\mathcal{S}_{m \pm}$, é interessante observar o que acontece com $\varepsilon$ atinge os limites de altas e baixas energias. Admitimos, em princípio, que $\varepsilon$ pode assumir valores

$$
\mathcal{E}_{m}=D \frac{1-\Lambda^{-1}}{\log \Lambda} \Lambda^{-m} .
$$

Como estamos interessados em distâncias $R$ superiores à escala atômica, usualmente $k R \gg 1$. Para $m$ pequeno, tal que $k_{F} R \mathcal{E}_{m} / D \gg 1$, as integrais à direita das equações (4.7) e (4.8) são muito pequenas, pois o integrando oscila rápido dentro dos intervalos $\mathcal{I}_{m \pm}$. Neste caso, procedemos com o cálculo numérico das integrais $\mathcal{S}_{m \pm}$ para $m<m_{0}$, onde $m_{0}$ é o menor inteiro satisfazendo a condição

$$
k_{F} R \mathcal{E}_{m_{0}} \gtrsim D
$$

Assim, encontrando $m_{0}$ a partir de (5.6), para todos os demais números $m \geq m_{0}$, é conveniente substituir $k R$ por $k_{F} R$ no denominador de $W(\varepsilon, R)$. Essa simplificação facilita o cálculo das integrais $\mathcal{S}_{m \pm}(R)$, que podem ser escritas como

$$
\mathcal{S}_{m \pm}=\frac{1}{k_{F} R} \int_{\mathcal{I}_{m \pm}} \sin (k R) d \varepsilon
$$


A integral (5.7) é solúvel analiticamente, resultando em

$$
\mathcal{S}_{m \pm}(R)=\frac{2 D}{\left(k_{F} R\right)^{2}} \sin \left[k_{F} R\left(1 \pm \frac{1-\Lambda^{-1}}{2} \Lambda^{-m}\right)\right] \sin \left[k_{F} R\left(\frac{1-\Lambda^{-1}}{2} \Lambda^{-m}\right)\right]
$$

ou ainda

$$
\mathcal{S}_{m \pm}(R)=\frac{2 D}{\left(k_{F} R\right)^{2}} \sin \left[k_{F} R\left(1 \pm \mathcal{E}_{m}\right)\right] \sin \left[k_{F} R \alpha_{m}^{2} / 2\right]
$$

onde usamos as relações (3.22) e (5.6) para os coeficientes $\alpha_{m}$ e $\mathcal{E}_{m}$.

Para $m \gg m_{0}$, podemos distinguir os coeficientes $\gamma_{n}$ pela paridade de $n$. Substituindo as somas $\mathcal{S}_{m \pm}$ expandidas em (5.9) na equação (4.9), obtemos

$$
\gamma_{n}(R)=\sum_{m=m_{0}}^{\infty} \frac{2 D}{\left(k_{F} R\right)^{2}}\left(\sin \left[k_{F} R\left(1+\mathcal{E}_{m}\right)\right]+(-1)^{n} \sin \left[k_{F} R\left(1-\mathcal{E}_{m}\right)\right]\right) \frac{u_{n m}}{u_{0 m}}
$$

onde, considerando a soma em $m$ uma constante, destacamos a amplitude $\gamma_{n}(R) \propto \frac{\sin \left(k_{F} R+\theta\right)}{\left(k_{F} R\right)^{2}}$ $\operatorname{com} \theta$ sendo uma fase.

A fase $\theta$, além de influenciar a amplitude de oscilação, nos permite associar à forma geral dos coeficientes $\gamma_{n}$ soluções pares e ímpares. No caso em que $\theta=n \pi \operatorname{com} n \in \mathbb{Z}$, os coeficientes $\gamma_{n}$ comportam-se como $\sin \left(k_{F} R\right)$ e, portanto, temos uma solução ímpar. Por outro lado, para $\theta=(n+1 / 2) \pi$ com $n \in \mathbb{Z}$, temos uma solução par $\gamma_{n} \propto \cos \left(k_{F} R\right)$.

Distinguir as soluções $\gamma_{n}$ pares e ímpares é equivalente a fixar o valor de $k_{F} R$ múltiplo de $\pi$ ou múltiplo de $\frac{\pi}{2}$. Para $k_{F} R=n \pi$, com $n \in \mathbb{Z}$ a equação (5.10) é exatamente

$$
\gamma_{n}(R)=\frac{4 D \cos \left(k_{F} R\right)}{\left(k_{F} R\right)^{2}} \sum_{m=m_{0}}^{\infty} \sin \left(\frac{k_{F} R \alpha_{m}^{2}}{2}\right) \sin \left(k_{F} R \mathcal{E}_{m}\right) \frac{u_{n m}}{u_{0 m}},
$$

enquanto para $k_{F} R=\left(n+\frac{1}{2}\right) \pi$, com $n$ inteiro, vale

$$
\gamma_{n}(R)=\frac{4 D \sin \left(k_{F} R\right)}{\left(k_{F} R\right)^{2}} \sum_{m=m_{0}}^{\infty} \sin \left(\frac{k_{F} R \alpha_{m}^{2}}{2}\right) \cos \left(k_{F} R \mathcal{E}_{m}\right) \frac{u_{n m}}{u_{0 m}} .
$$

Em particular, para $m$ suficientemente grande, os valores de $\mathcal{E}_{m}$ e $\alpha_{m}$ são cada vez menores. Nesta situação, como $k_{F} R \mathcal{E}_{m} \ll 1$, podemos expandir as expressões (5.11) e (5.12) em

$$
\gamma_{n}(R) \approx( \pm 1) 4 D \sum_{m}^{\infty} \mathcal{E}_{m} u_{0 m} u_{n m} \approx( \pm 1) 4 D \sum_{m}^{\infty} u_{1 m} u_{n m}
$$

e

$$
\gamma_{n}(R)=\frac{ \pm 4 D}{k_{F} R} \sum_{m}^{\infty} u_{0 m} u_{n m}
$$



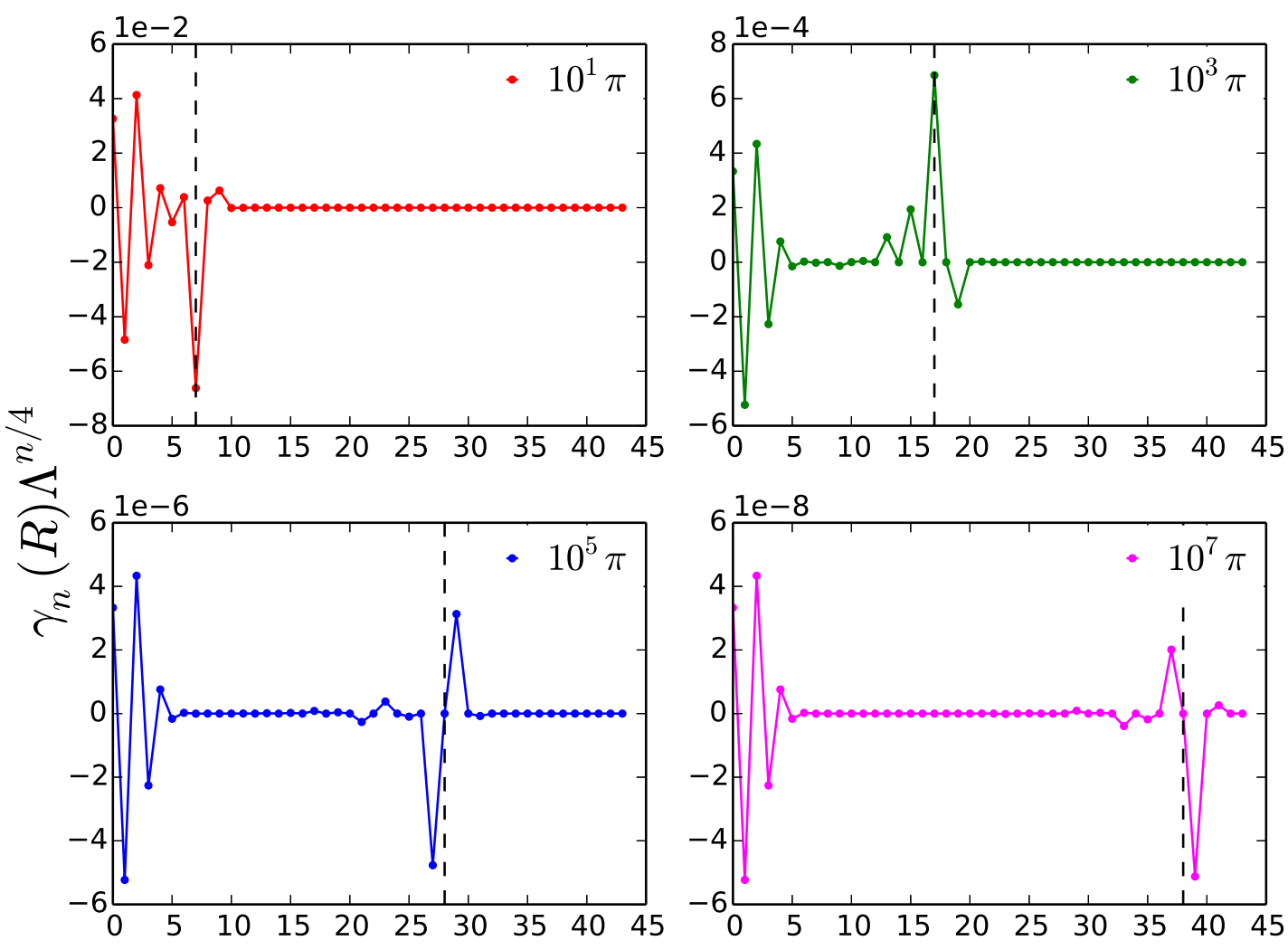

$n$

Figura 5.2 - Coeficientes $\gamma_{n}(R)$ para $k_{F} R=n \pi$ com $n \in[1000,100,10,1]$. O número $n$ de iteração característico $\mathcal{N}_{k_{F} R}$ no qual $\gamma_{n}$ é máximo aparece destacado para cada um dos casos mostrados na figura. Fonte: elaborada pela autora.

Nas figuras 5.2 e 5.3 mostramos os coeficientes $\gamma_{n}(R)$ como função de $n$ para $k_{F} R=n \pi$ e $k_{F} R=(n+1 / 2) \pi$ com $r \in[1000,100,10,1]$. Estes resultados foram obtidos calculando numericamente as integrais $\mathcal{S}_{m \pm}$ através da regra de Simpson. O parâmetro de discretização escolhido foi $\Lambda=3.0$

Em geral, observamos que há um valor $n$ característico no qual o coeficiente $\gamma_{n}$ é máximo e, portanto, maior será sua contribuição associada aos $\phi_{\mu}(R)$. Esses coeficientes podem ser calculados com a relação

$$
\mathcal{N}_{k_{F} R}= \begin{cases}\left\lceil\frac{\log \left(k_{F} R\right)}{\log (\sqrt{\Lambda})}\right], & \text { se } k_{F} R=n \pi \\ \left\lceil\frac{\log \left(k_{F} R\right)}{\log (\sqrt{\Lambda})}\right]+1, & \text { se } k_{F} R=\left(n+\frac{1}{2}\right) \pi\end{cases}
$$

onde $\lceil x\rceil$ denota a parte inteira de $x$ arredondada para cima, ou o teto de $x$. 

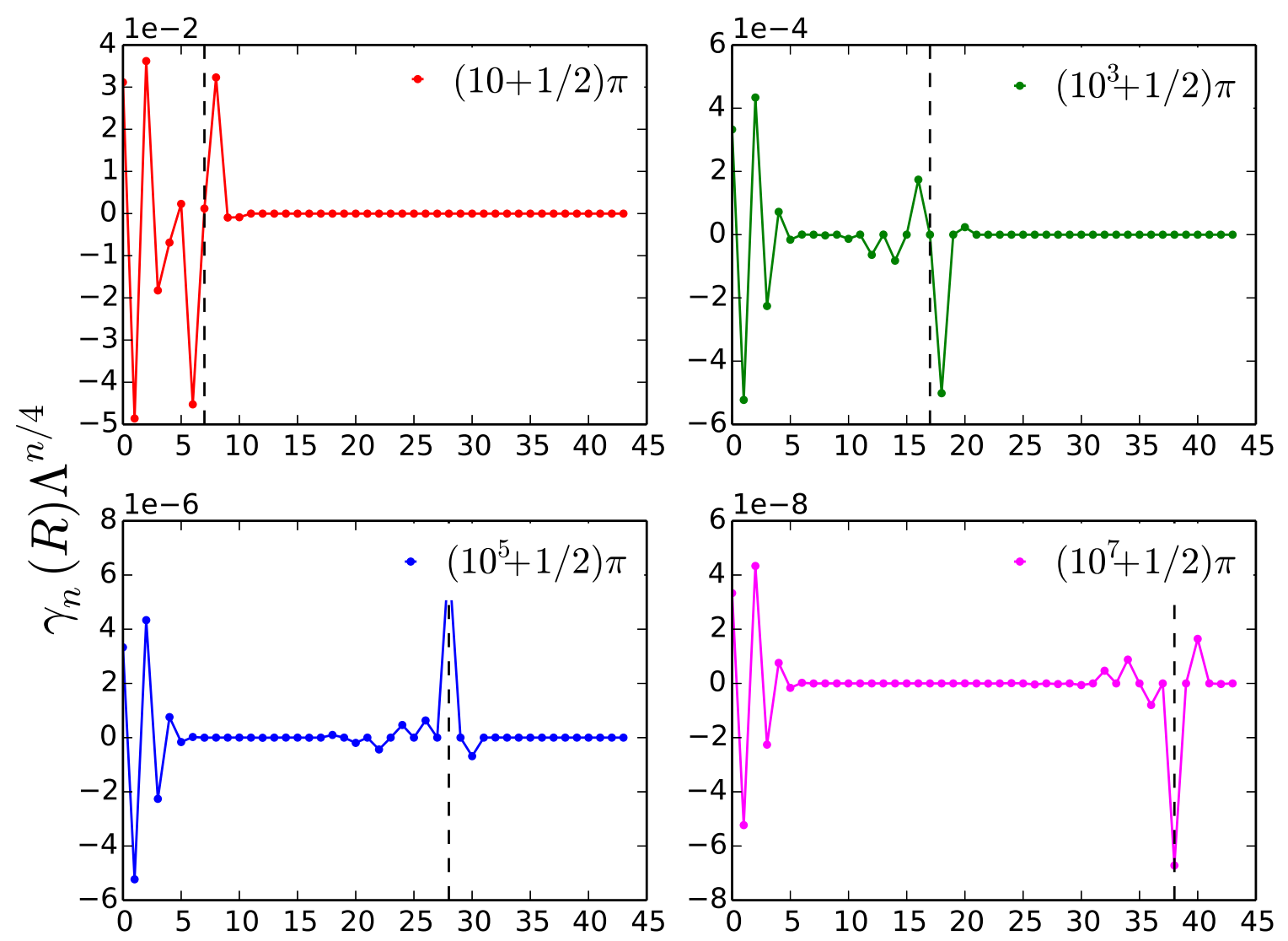

$n$

Figura 5.3 - Coeficientes $\gamma_{n}(R)$ para $k_{F} R=\left(n+\frac{1}{2}\right) \pi$ com $n \in[1000,100,10,1]$. Para cada valor de $k_{F} R$ destacamos a iteração característica $\mathcal{N}_{k_{F} R}$, calculada através da equação (5.15). Conforme $k_{F} R$ fica menor, a contribuição dos coeficientes $\gamma_{0}$ vai se tornando cada vez maior, superando o pico típico em $n=\mathcal{N}_{k_{F} R}$. Comparando estes resultados com a figura 5.2, notamos que esse efeito é mais proeminente para valores $k_{F} R$ múltiplos de $\frac{\pi}{2}$, conforme esperado pela expansão dos $\gamma_{n}$ na equação (5.10). Fonte: elaborada pela autora.

Em primeira aproximação, poderíamos calcular $\mathcal{N}_{k_{F} R}$ conforme a equação (5.15) e considerar somente o coeficiente $\gamma_{n}$ associado a $\mathcal{N}_{k_{F} R}$, desprezando os demais termos. Contudo, para o caso em que $k_{F} R$ é um múltiplo inteiro de $\frac{\pi}{2}$ essa aproximação não seria boa: o coeficiente $\gamma_{0}$ vai aumentando a medida que diminuimos $k_{F} R$ e supera os demais $\gamma_{n}(n=1, \ldots, N)$, como podemos observar na figura 5.3.

Em suma, as expansões (5.11) e (5.12), bem como a identificação dos valores de $\mathcal{N}_{k_{F} R}$ nos quais $\gamma_{n}$ é máximo, são ilustrativas para a análise de propriedades que possam ser mapeadas no operador $\phi_{\mu}(R)$. Isto será exemplificado na seção subsequente com a taxa de relaxação $\left(1 / T_{1}\right)_{\text {vet }}$. 


\subsection{A taxa de relaxação associada à contribuição ve- torial}

No capítulo 4, mostramos que a contribuição dos termos cruzados $f_{n}^{\dagger} \bar{c}_{\varepsilon}\left(\bar{c}_{\varepsilon}^{\dagger} f_{n}\right)$ depende somente dos elementos de matriz $\left\langle\varphi\left|f_{n}^{\dagger}\right| \psi\right\rangle$ multiplicados por uma amplitude dependente das energias e da distância $R$. A taxa de relaxação devida a esta contribuição, que denominamos vetorial, tem a forma

$$
\left(\frac{1}{T_{1}}\right)_{v e t} \propto \frac{4 \pi}{\hbar Z} \sum_{|\varphi\rangle,|\psi\rangle}\left(1-W^{2}\left(k_{\Delta E} R\right)\right) \frac{\left|\sum_{n} \gamma_{n}(R)\left\langle\varphi\left|f_{n}^{\dagger}\right| \psi\right\rangle\right|^{2}}{e^{\beta E_{\varphi}}+e^{\beta E_{\psi}}},
$$

onde $|\varphi\rangle$ e $|\psi\rangle$ são autoestados do Hamiltoniano $H_{N}, k_{\Delta E}=k_{F}(1+\Delta E / D)$ e $\Delta E=$ $E_{\varphi}-E_{\psi}$.

Ao longo das iterações, as energias $\varepsilon$ dos autovalores $E_{\varphi^{\prime}}$ e $E_{\psi^{\prime}}$ vão ficando cada vez menores, já que estão essencialmente associadas à temperatura do sistema. Quando $T<T_{k}$ estamos no regime de baixas energias, que é exatamente a zona de interesse relacionada à manifestação do efeito Kondo.

Para um $R$ fixo e $\varepsilon$ pequeno recaímos na aproximação (5.7) para as integrais $\mathcal{S}_{m \pm}$. Nesta situação, podemos estudar o comportamento da função seno no limites de baixas energias. Fazendo a expansão para $\varepsilon$ pequeno, segue que

$$
\sin (k R) \approx \begin{cases}\sin \left(k_{F} R\right)\left(1-\frac{\left(k_{F} R\right)^{2}}{2} \frac{\varepsilon^{2}}{D^{2}}\right) & \text { se } k_{F} R=\left(n+\frac{1}{2}\right) \pi \\ \left(k_{F} R \frac{\varepsilon}{D}\right) \cos \left(k_{F} R\right) & \text { se } k_{F} R=n \pi\end{cases}
$$

onde $n \in \mathbb{Z}$.

Assim como os coeficientes $\gamma_{n}$, a separação das soluções pares e ímpares na expansão (5.17) nos permite diferenciar diferentes comportamentos conforme o valor de $k_{F} R$ é múltiplo de $\pi$ ou de $\pi / 2$. Uma vez que a taxa $\left(1 / T_{1}\right)_{\text {vet }}$ está associada à interferência entre os estados acoplados com a ponta de prova e com a impureza, devemos ser capazes de identificar os pontos $\pi$ e $\pi / 2$ com os casos de interferência construtiva ou destrutiva. Para tanto, vamos inicialmente analisar as oscilações de Friedel da taxa $\left(1 / T_{1}\right)_{\text {vet }}$ em função da distância $k_{F} R$. Em seguida, mostraremos qual a dependência da taxa de relaxação com a temperatura para os casos de interferência construtiva, destrutiva e para um caso onde não há oscilação de Friedel. Nessa dissertação, mostraremos os resultados para o modelo de Anderson simétrico. Generalizações para o caso assimétrico serão contempladas em trabalhos futuros. 
Os parâmetros de entrada do programa são apresentados na tabela 5.1, onde $D$, $\varepsilon_{d}$ e $U$ denotam as energias do Hamiltoniano de Anderson; $\Lambda$ é o parâmetro de discretização; $E_{\text {min }}$ corresponde à temperatura limitando do número $N$ de iterações; $E_{U V}$ é a energia de corte para o truncamento de estados; $\beta_{0}$ denota a temperatura inicial a partir da qual o sistema é resfriado.

Tabela 5.1 - Parâmetros de entrada do código adotados no cálculo da taxa de relaxação $\left(1 / T_{1}\right)_{v e t}$.

\begin{tabular}{|c|c|c|c|c|c|c|}
\hline \hline$D$ & $\varepsilon_{d}$ & $U$ & $\Lambda$ & $E_{\min }$ & $E_{U V}$ & $\beta_{0}$ \\
\hline 1.0 & -15.0 & 30.0 & 2.4 & $10^{-11}$ & 27.0 & 0.4 \\
\hline \hline
\end{tabular}

Fonte: elaborada pela autora.

Uma vez escolhidos os parâmetros para o modelo de Anderson e para o NRG, computamos o cálculo da taxa $\left(1 / T_{1}\right)_{v e t}$ e estudamos seu comportamento como função da temperatura $T$ e da distância $R$, considerando a última no conjunto $k_{F} R=[n \pi,(n+1 / 2) \pi,(n+1 / 4) \pi]$, com $n \in \mathbf{Z}$. Nessas condições, a temperatura de Kondo foi calculada* como $k_{b} T_{K}=1.2512 \times 10^{-5}$, de modo que o raio de Kondo associado seria $R_{K} \approx 729 \times 10^{4}$.

Inicialmente, analisamos a componente vetorial como função da distância. Na figura 5.4 mostramos as oscilações de Friedel de $\left(1 / T_{1}\right)_{\text {vet }}$ no ponto fixo de baixa temperatura.

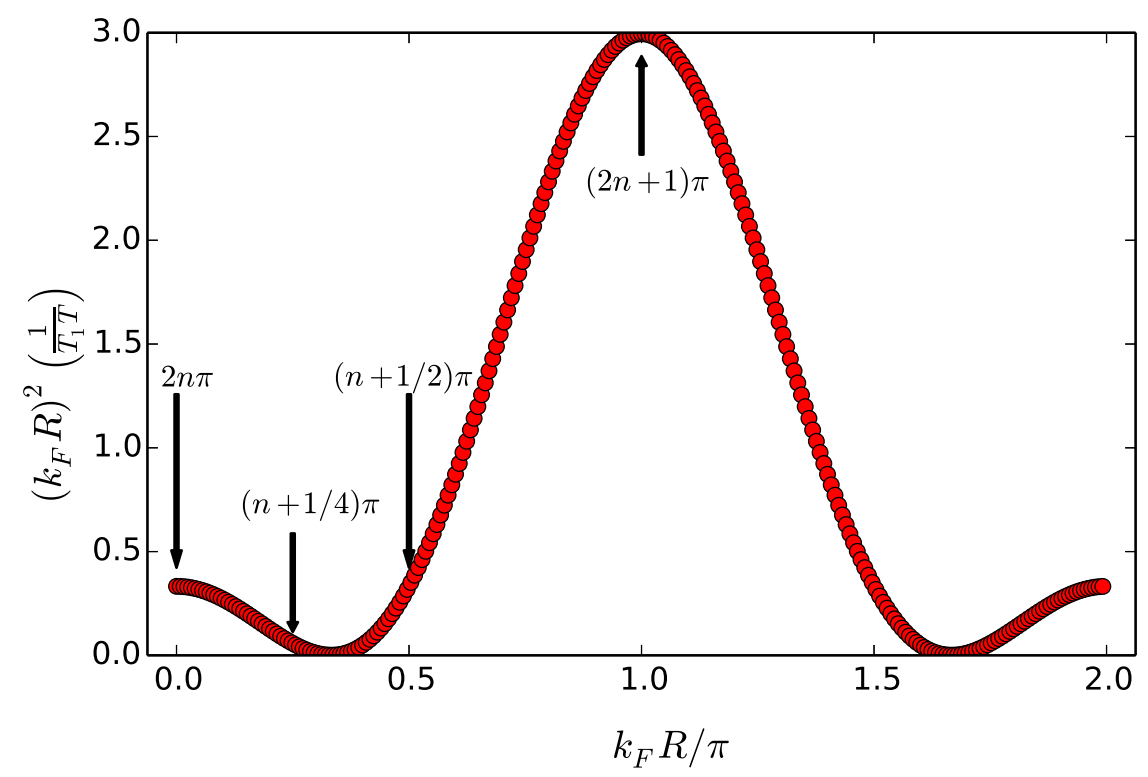

Figura 5.4 - Oscilações de Friedel no ponto fixo de temperatura $T \ll T_{K}$. Pontos destacados com setas correspondem aos máximos e aos nós das oscilações. O ponto $(n+1 / 4) \pi$ combina os comportamentos destrutivo e construtivo. Fonte: elaborada pela autora.

*Usamos a definição da condutância para calcular $T_{K}$ 
A curva apresentada na figura 5.4 representa as oscilações da taxa de relaxação quando $T \ll T_{K}$. O período das oscilações é $2 \pi$ com os máximos ocorrendo em $(2 n+1) \pi$ (múltiplos inteiros de $\pi$ ) e os mínimos próximos a $\pi / 4$. Os nós das oscilações equivalem a pontos associados a distâncias na forma $2 n \pi$ e $(n+1 / 2) \pi$. O ponto $(n+1 / 4) \pi$ é ilustrativo para analisar distâncias que combinam ambos os comportamentos destrutivo e construtivo da relaxação $\left(1 / T_{1}\right)_{\text {vet }}$. Assim, em seguida, vamos analisar as dependências térmicas dos nós da oscilação $2 n \pi$ e $(n+1 / 2) \pi$ e de pontos da forma $(n+1 / 4) \pi$.

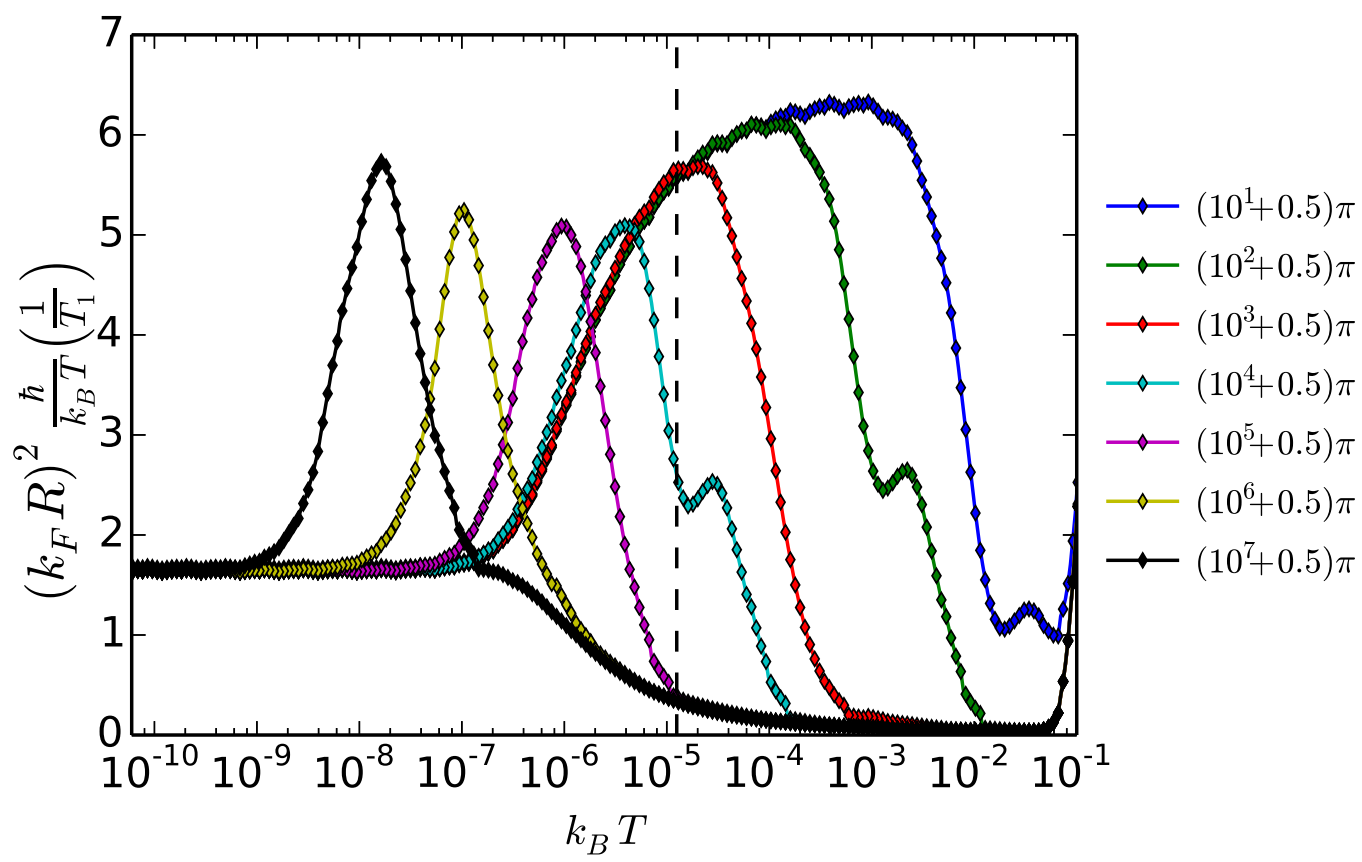

Figura 5.5 - Contribuição vetorial para a taxa de relaxação $1 / T_{1}$ com $k_{F} R=\left(n+\frac{1}{2}\right) \pi$. A correspondente parcela da taxa é proporcional a $\left(k_{F} R\right)^{2}$ e decai para baixas temperaturas. A pequenas distâncias, no limite de altas temperaturas, a curva pode ser mapeada na condutância $\mathcal{G}_{\text {side }}$ de um dispositivo formato $\mathrm{T}$. Fonte: elaborada pela autora.

Com relação à dependência térmica de $\left(1 / T_{1}\right)_{\text {vet }}$ para distâncias fixas, podemos isolar os casos $k_{F} R=(n+1 / 2) \pi$ e $k_{F} R=n \pi$ e mostrar o que acontece quando resfriamos o sistema. A figura 5.5 ilustra a transição entre o ponto de interferência de construtiva para destrutiva como função da temperatura para distâncias $k_{F} R=(n+1 / 2) \pi$ com $n \in$ $\left[10,10^{2}, 10^{3}, 10^{4}, 10^{5}, 10^{6}, 10^{7}\right]$. No regime de altas temperaturas, observamos que as curvas associadas às menores distâncias tendem à mesma curva uninversal descrevendo a condutância 
$\mathcal{G}_{\text {side }}$ para o dispositivo em formato $T^{\dagger}$ No limite $T \rightarrow 0$, assim como a condutância, a taxa de relaxação decai rapidamente, mas atinge um patamar não-nulo.

Por outro lado, para a transição entre a interferência destrutiva para construtiva (distâncias $k_{F} R=n \pi$, com $n \in \mathbb{Z}$ ), a taxa de relaxação começa baixa e vai aumentando até atingir um patamar constante, como mostramos na figura 5.6. Analogamente ao caso anterior, podemos identificar um comportamento semelhante ao da curva universal que descreve a condutância $\mathcal{G}_{S E T}$ do transístor de um elétron.

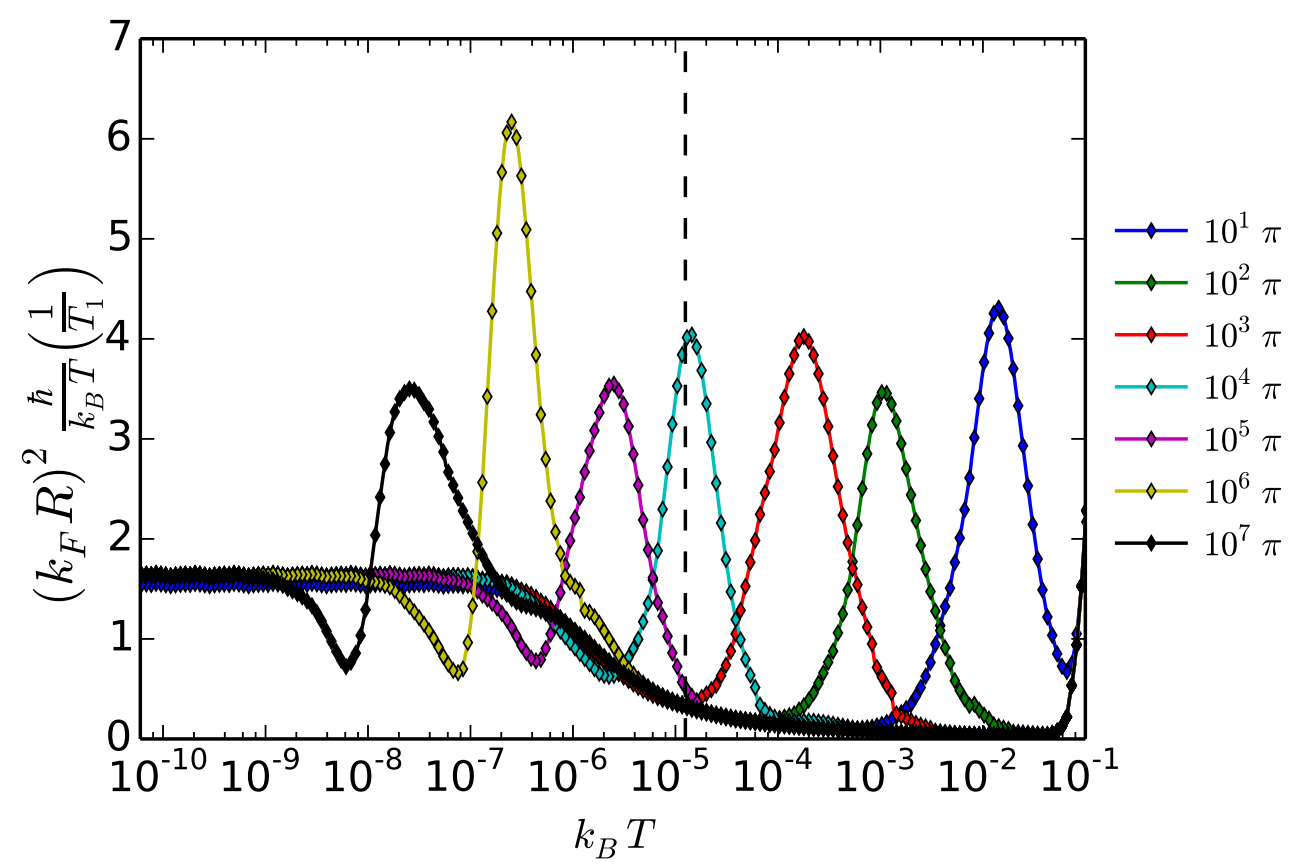

Figura 5.6 - Contribuição para a taxa de relaxação $1 / T_{1}$. com $k_{F} R=n \pi$. A contribuição começa muito pequena para altas temperaturas e aumenta até atingir um patamar constante em $T<T_{K}$. O comportamento global é análogo ao da condutância $\mathcal{G}_{S E T}$ de um transístor de um elétron. Fonte: elaborada pela autora.

Nos nós das oscilações (figuras 5.5 e 5.6, respectivamente), observamos uma mudança no comportamento térmico de $\left(1 / T_{1}\right)_{\text {vet }}$ quando ultrapassamos a temperatura de Kondo e, consequentemente, o raio de Kondo $R_{K}$. Esse efeito é mais proeminente no caso em que o nó da oscilação marca uma transição de interferência construtiva para destrutiva, especificamente quando as curvas $R>R_{K}$ passam a se destacar do comportamento universal análogo à condutância $\mathcal{G}_{\text {side }}$. Um efeito interessante diz respeito aos picos que ocorrem a diferentes temperaturas para cada uma das distâncias consideradas e a partir dos quais começamos a

†Os detalhes envolvendo o cálculo da condutância $\mathcal{G}_{\text {side }}$ side-coupled e seu resultado numérico são apresentados no apêndice $C$. 
observar os efeitos da relaxação. Esses máximos estão associados ao comprimento térmico de de Broglie $\lambda_{B}=2 \pi \hbar v_{F} / k_{B} T$, de modo que para distâncias $R>\lambda_{B}$ estamos em uma região cega aos efeitos quânticos e, assim, não há sinal de relaxação magnética. Uma vez imersos na região $R<\lambda_{B}$, o sinal associado à $\left(1 / T_{1}\right)_{\text {vet }}$ torna-se visível e conseguimos capturar a inteferência entre os estados acoplados à impureza e à ponta de prova.

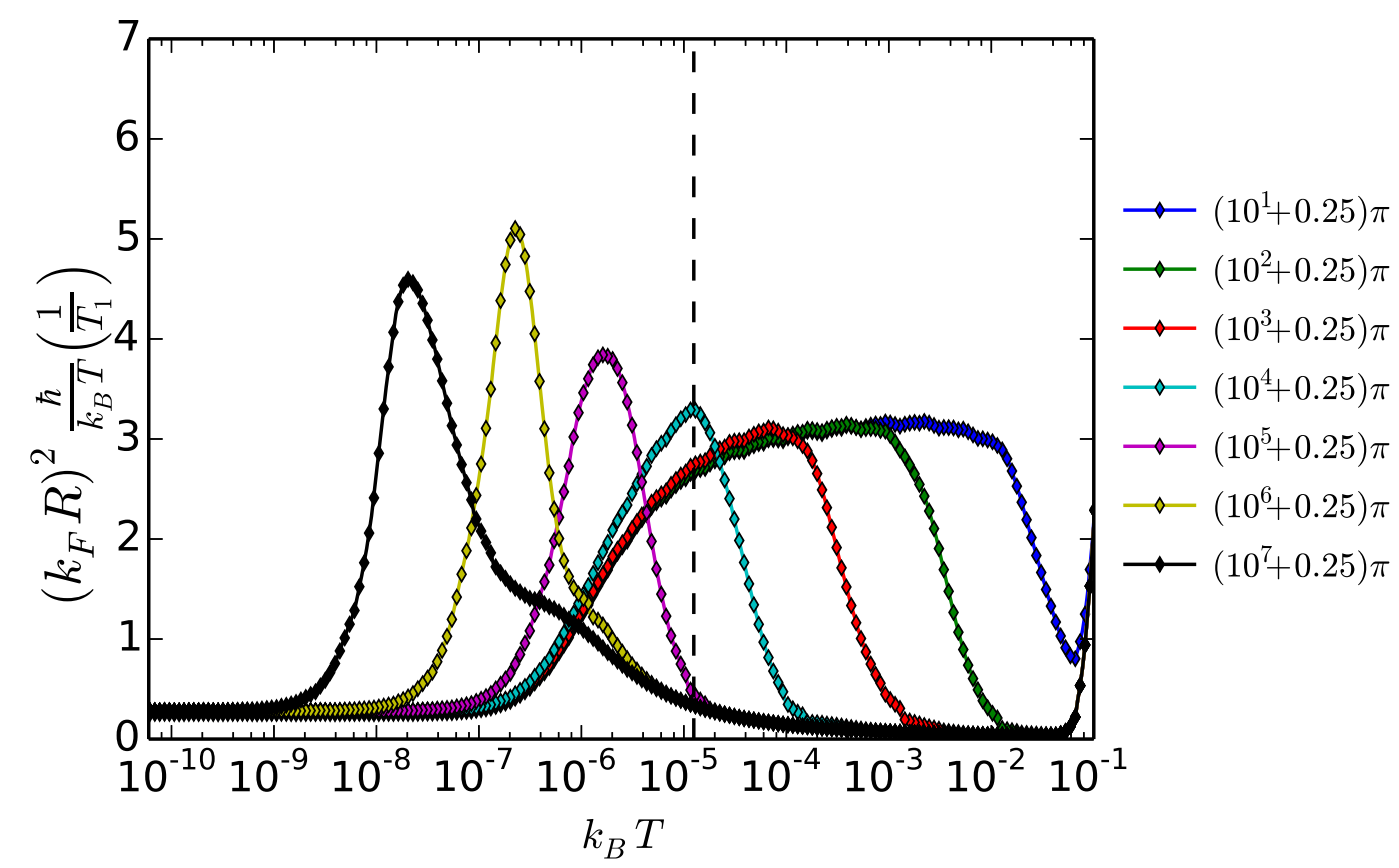

Figura 5.7 - Taxa de relaxação $\left(1 / T_{1}\right)_{\text {vet }}$ em função da temperatura na ausência de oscilação. A altas temperatuas, a componente vetorial de $1 / T_{1}$ mescla os comportamentos das condutâncias $\mathcal{G}_{\text {side }}$ e $\mathcal{G}_{S E T}$. A mudança de comportamento ocorre nas vizinhanças do raio da nuvem Kondo $R_{K}=\hbar v_{F} / k_{B} T_{K}$, confirmando a hipótese de que o raio seria encontrado em uma escala de distância mesoscópica. Fonte: elaborada pela autora.

Voltando à figura 5.4, nos resta analisar a dependência térmica da taxa de relaxação para pontos onde temos uma mistura dos comportamentos destrutivo e construtivo, ou seja, para distâncias $k_{F} R=(n+\pi / 4)$, com $n \in \mathbb{Z}$. Na figura 5.7 apresentamos a taxa $\left(1 / T_{1}\right)_{\text {vet }}$ como função da temperatura para pequenas e grandes distâncias. Podemos observar a transição entre os comportamentos das figuras 5.5 e 5.6 para $R>R_{K}$ e $R<R_{K}$, respectivamente. Em outras palavras, dentro na nuvem, após passarmos pela temperatura associada ao pico de de Broglie, a taxa de relaxação decai até atingir o patamar constante. Fora da nuvem, por outro lado, a taxa começa próxima de zero e atinge o patamar constante após passar pelo pico de de Broglie. Inferimos que na ausência de oscilação, a mudança de comportamento da taxa $\left(1 / T_{1}\right)_{\text {vet }}$ permite capturar o efeito da nuvem Kondo na ponta de prova, pois a taxa 
de relaxação pressupostamente assume formas diferentes quando estamos dentro ou fora da nuvem, de modo que que a transição entre os dois comportamentos se dá na vizinhança de $R_{K}$. Esse resultado ratifica a validade da relação $R_{K}=\hbar v_{F} / k_{B} T_{K}$ e, consequentemente, confirma a hipótese de que o raio da nuvem Kondo seria encontrado na escala mesoscópica.

Finalmente, podemos sumarizar nossos resultados na figura 5.8, na qual identificamos as distâncias correspondentes ao interior e ao exterior da nuvem, bem como as redondezas de $R_{K}$. Dentro da nuvem, ou seja, para $R \ll R_{K}$ a dependência de $\left(1 / T_{1}\right)_{\text {vet }}$ pode ser mapeada na curva universal que descreve a condutância universal $\mathcal{G}_{\text {side }}$. Por outro lado, para distâncias suficientemente grandes, tais que $R \gg R_{K}$, a relaxação vetorial em função da temperatura é análoga à curva da condutância $\mathcal{G}_{S E T}$. Uma observação importante é que a mudança entre esses comportamentos não é súbita: entre $10^{3}<k_{F} R / \pi<10^{6}$ o comportamento térmico análogo às condutâncias fica mascarado pelo pico de de Broglie.

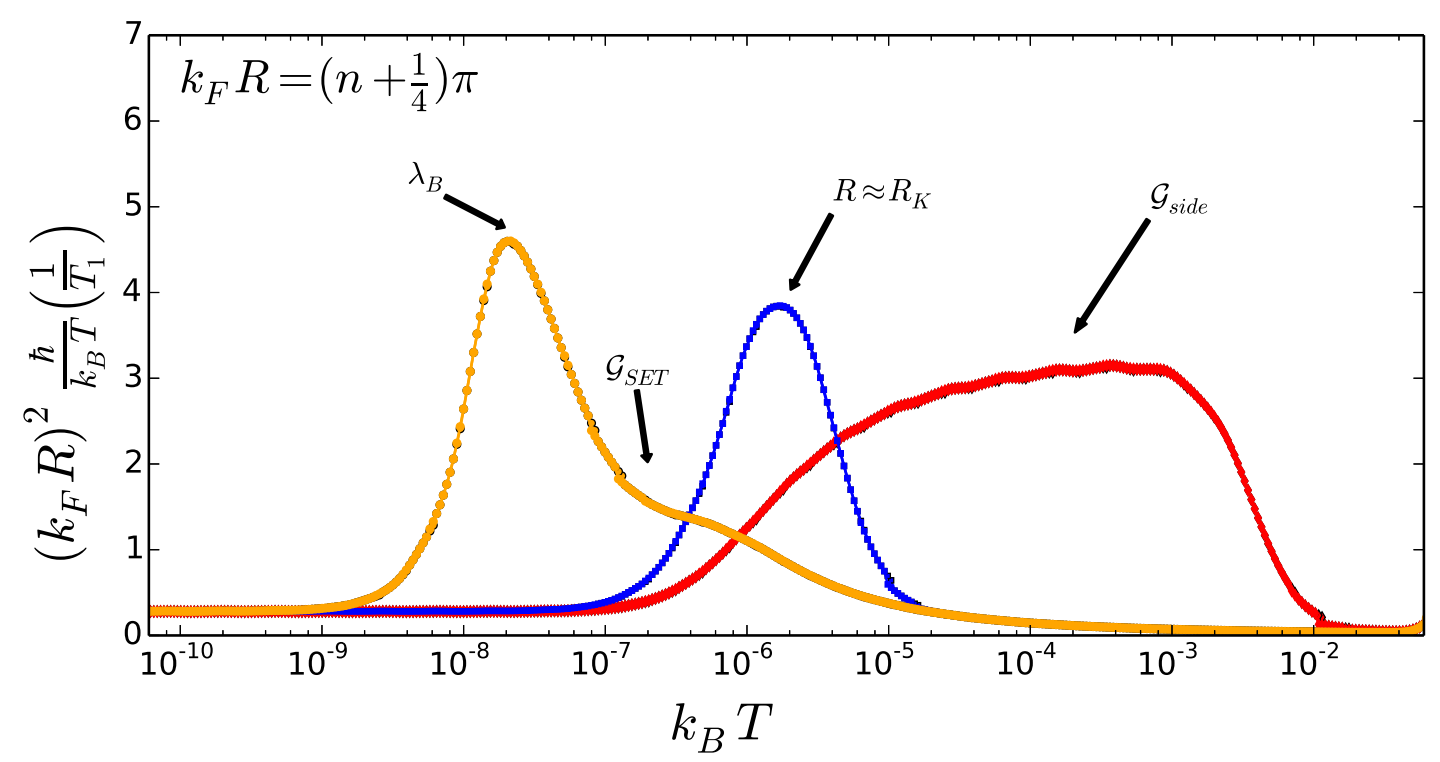

Figura 5.8 - Taxa de relaxação em função da temperatura e os limites da nuvem Kondo. Para $R \gg R_{K}$ a dependência térmica é análoga a da curva $\mathcal{G}_{S E T}$, enquanto para $R \ll R_{K}$ ela pode ser mapeada na condutância $\mathcal{G}_{\text {side }}$. Fonte: elaborada pela autora.

Matemática e fisicamente, nossos resultados têm um paralelo físico com as condutâncias observadas em nanoestrutras de geometria $T$ e no transístor de um único elétron $(19,20)$. Nesses dispositivos um potencial controla o número quântico de ocupação no ponto, favorecendo ou bloqueando a passagem de corrente. Quando o potencial favorece o transporte através do fio, a condutância cresce rapidamente de zero, a temperaturas baixas, até um patamar, a altas temperaturas. Por outro lado, quando o potencial favorece o ponto - que neste 
caso funciona como uma impureza Kondo - a condutância decai para baixas temperaturas devido ao bloqueio à passagem de carga através do fio.

O efeito capturado pela taxa de relaxação $\left(1 / T_{1}\right)_{\text {vet }}$ é a interferência entre estados acoplados à impureza - descritos pelos operadores $f_{n}$ - e os estados ortogonais aos últimos descritos pelos operadores $\bar{c}_{\varepsilon}$. Podemos interpretar fisicamente a taxa de relaxação da contribuição vetorial como um efeito de espalhamento cruzado, isto é, um elétron do canal da banda de condução associado ao orbital $\phi_{n}$ é espalhado pela impureza e alcança o canal dos orbitais $\bar{c}_{\varepsilon}$ da ponta de prova invertendo seu spin. A volta também é válida: um elétron do canal da ponta de prova troca um estado de momento magnético com o canal da banda de condução. Esse efeito é análogo ao que observamos com a condutância em pontos quânticos para ambas as geometrias. Nesses sistemas há também dois canais - um canal par acoplado ao ponto quântico e outro canal ímpar desacoplado - que dependem da simetria de inversão em torno do ponto quântico. A condutância exige a transferência de elétrons entre os canais. Assim, não é surpreendente que a curva da taxa de relaxação siga um comportamento universal semelhante.

Uma vez que nesta dissertação focamos nossa atenção em distâncias grandes, nas quais a contribuição do termo matricial é pequena, não vamos apresentar os resultados numéricos da taxa $\left(1 / T_{1}\right)_{m a t}$. No entanto, deixamos aqui o procedimento analítico e o algoritmo para o cálculo da mesma - os quais serão úteis quando as distâncias de interesse forem muito pequenas ou ainda para geometrias mais complexas, nas quais efeitos de superfície tornam-se importantes.

O efeito de todos os $N^{2}$ elementos de matriz na taxa total $\left(1 / T_{1}\right)_{\text {mat }}$ é, portanto, uma questão que deverá ser analisada em trabalhos futuros dedicados à relaxação para distâncias $R$ pequenas (nas quais a ponta de prova está muito próxima da impureza). Com essa nova abordagem, verificaremos se a taxa de relaxação ESR é, de fato, uma técnica capaz de fornecer algum indício do raio $R_{K}$ da nuvem Kondo.

\footnotetext{
${ }^{\ddagger}$ Apêndice B e seção 4.2.2.
} 


\section{Conclusão}

Na presente dissertação estudamos a taxa de relaxação longitudinal $1 / T_{1}$ de uma ponta de prova magnética em uma liga metálica de geometria especial. Considerando um metal semi-infinito na direção $z$ com uma impureza localizada a uma distância $d$ do plano $z=0$, propusemos analisar a taxa $1 / T_{1}$ como função da temperatura $T$ e da distância $R$ entre a ponta de prova e a impureza. A motivação para este procedimento remete ao alcance da polarização de elétrons ao redor da impureza, cuja dinâmica poderia afetar a relaxação do sistema e, assim, nos forneceria indícios sobre a formação a chamada nuvem Kondo.

No capítulo 1 contextualizamos historicamente o problema Kondo e recapitulamos os principais resultados que contribuíram para o estudo de impurezas magnéticas em metais desde as primeiras formulações até a atualidade. Discorremos sobre avanços teóricos (NRG) e experimentais (STM) que permitiram o estudo do efeito Kondo e abriram caminho para a investigação de problemas correlatos e questões indefinidas, destacando a medida da nuvem Kondo.

No segundo capítulo introduzimos o sistema quântico de nosso interesse, apresentando os Hamiltonianos envolvidos. Usamos o modelo de Anderson para descrever o sistema metálico (equação (2.1)) e o Hamiltoniano $H_{\text {probe }}$ para a interação do sistema com a ponta de prova (equação (2.10)). Definimos a base contínua $\left\{c_{\varepsilon}, \overline{c_{\varepsilon}}\right\}$ (equações (2.11) e (2.16)) e

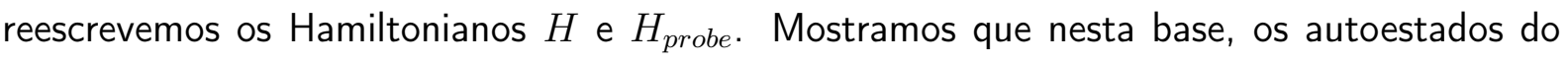
Hamiltoniano perturbado $\tilde{H}$ (relação (2.28)) são desacoplados, o que simplifica o tratamento analítico dos estados ortogonais à impureza (autoestados do operador $\bar{c}_{\varepsilon}$ ).

No capítulo 3 apresentamos o método do Grupo de Renormalização Numérico (NRG), versando sobre seu sucesso no problema Kondo. Detalhamos as etapas envolvidas na implementação do NRG, com especial atenção à diagonalização iterativa do Hamiltoniano de Anderson. Ilustramos como a base primitiva $\left\{\left|q, s, s_{z}, r\right\rangle\right\}$ de estados é construída na iteração $N$ (conjunto de definições (3.53) a (3.56)), além de abordar o cálculo de elementos de matriz $\left\langle q^{\prime}, s^{\prime}, s_{z}^{\prime}, r\left|f_{n}^{\dagger}\right| q, s, s_{z}, r\right\rangle$ necessários para o cálculo de propriedades físicas. Pudemos aplicar o método NRG para a diagonalização do nosso modelo obtendo dados numéricos para computar a taxa de relaxação magnética. 
Nosso quarto capítulo foi dedicado à taxa de relaxação magnética longitudinal $1 /\left(T_{1}\right)$. Começamos definindo a expressão para a taxa $1 /\left(T_{1}\right)$ com base na transição de estados quânticos (definição (4.1)) mediada por $H_{\text {probe }}$. Tendo definido o operador $\phi_{\mu}(R)$ (apresentado em (2.24)) como combinação dos estados de Lanczos (equação (4.3)), fomos capazes de obter uma expressão analítica para a taxa de relaxação magnética na base do NRG.

Ainda no capítulo 4, mostramos que a taxa de relaxação poderia ser decomposta em três contribuições, as quais foram denominadas escalar, vetorial e matricial. O termo escalar foi associado aos estados de $\Phi_{0 \mu}(R)$ (ou $\bar{c}_{\varepsilon}$ ) e, assim, completamente desacoplado do sistema não-perturbado. Independente da temperatura e da polarização de spin envolvida na formação da nuvem Kondo, este termo não foi considerado na análise da taxa de relaxação, pois funciona como um termo residual que soma à taxa total uma constante.

A contribuição dos termos cruzados $\bar{c}_{\varepsilon}^{\dagger} f_{n}$ e $f_{n}^{\dagger} \bar{c}_{\varepsilon}$ foi denominada vetorial. A justificativa para a escolha dessa terminologia deveu-se ao tipo de dependência que a taxa associada ao termo cruzado apresenta. Neste caso, ela requer o cálculo do vetor de elementos de matriz $\left\langle\varphi\left|f_{n \mu}^{\dagger}\right| \psi\right\rangle(n=0, \ldots, N)$ (expressão final apresentada em (4.20)). Relacionamos o cálculo desta contribuição à computação dos elementos de matriz apresentada na seção 3.6.4.

A taxa de relaxação devida apenas a estados da banda de condução e da impureza (que são autoestados dos operadores $f_{n}$ ) foi atribuída ao termo que denotamos matricial. Analogamente ao termo vetorial, a contribuição matricial recebe este nome por envolver uma matriz de elementos $\left\langle\varphi\left|f_{n \uparrow}^{\dagger} f_{m \downarrow}\right| \psi\right\rangle(n, m=0, \ldots, N)$ (equação (4.21)). Tendo identificado os estados $|\varphi\rangle|\psi\rangle$ contribuindo para a relaxação como àqueles pertencendo ao mesmo setor $(q, s)$, prosseguimos buscando um método eficiente e acurado para determinar os elementos de matriz da forma $\left\langle\varphi\left|f_{n \uparrow}^{\dagger} f_{m \downarrow}\right| \psi\right\rangle(n, m=0, \ldots, N)$ —tarefa que constituiu uma das principais contribuições técnicas desta dissertação. A contribuição do termo matricial diminui rapidamente com a distância e, por isso, não apresentamos resultados numéricos para $(1 / T 1)_{\text {mat }}$. No entanto, deixamos aqui registrado o procedimento que permite calculá-lo, o qual servirá para descrever medidas de STM nas vizinhanças de impurezas magnéticas.

Na seção 4.2.2 discutimos duas formas para implementar o cálculo dos elementos matriciais. A primeira delas, a completeza, foi testada numericamente para diversos parâmetros do modelo, falhando justamente no regime de interesse $\left(T<T_{K}\right)$. Apresentamos no apêndice $\mathrm{A}$ os detalhes do cálculo dos elementos $\left\langle\varphi\left|f_{n \uparrow}^{\dagger} f_{m \downarrow}\right| \psi\right\rangle(n, m=0, \ldots, N)$ através da completeza, enfatizando suas imprecisões numéricas e mostrando porque ele é inadequado. O principal problema com a completeza é o truncamento de estados, que afeta dramaticamente as últimas iterações. 
A segunda alternativa, também mostrada no capítulo 4 consistiu na determinação de relações de recorrência e culminou em uma expressão relacionando os elementos $\left\langle\varphi\left|f_{n \uparrow}^{\dagger} f_{m \downarrow}\right| \psi\right\rangle$ aos vizinhos $\left\langle\varphi\left|f_{n \pm 1 \uparrow}^{\dagger} f_{m \pm 1 \downarrow}\right| \psi\right\rangle$ (relação (4.30)). Fomos capazes de demonstrar que determinando a linha $n=N$ e a coluna $m=0$, poderíamos aplicar a relação de recorrência para determinar todo o restante dos elementos matriciais (figuras 4.2 e 4.1). No apêndice B apresentamos como calcular estes elementos por rotações - o que exige muito mais cuidado do que o cálculo de elementos vetoriais, dada a necessidade de armazenar elementos das iterações precedentes e computar alguns invariantes não-diagonais.

No capítulo 5 analisamos o comportamento dos coeficientes $u_{m n}$ e $\gamma_{n}$ envolvidos nas transformações dos operadores $\phi_{\mu}(R)$ na base de Lanczos. Analisamos o comportamento assintótico dos $u_{n m}$ com auxílio da função geradora $U_{n}(z)$ (equação (5.2)). Expandindo os coeficientes $\gamma_{n}$ e usando uma aproximação para as somas $\mathcal{S}_{m \pm}$ no limite de $m$ grande, pudemos mostrar que eles podem ser decompostos em termos de soluções pares e ímpares conforme o valor de $k_{F} R$. Exploramos esta propriedade graficando $\gamma_{n}$ como função de $n$ para $k_{F} R=p \pi$ e $k_{F} R=\left(p+\frac{1}{2}\right) \pi$, com $p \in \mathbb{Z}$ (figuras 5.2 e 5.3). Encontramos um número $n$ característico no qual $\gamma_{n}$ é máximo e observamos que o coeficiente $\gamma_{0}$ é dominante.

A segunda parte do quinto capítulo foi dedicada à análise de resultados numéricos obtidos para a taxa de relaxação da contribuição vetorial como função da temperatura e da distância. Mostramos que esse termo corresponde à interferência entre os estados acoplados à impureza e à ponta de prova. Acompanhamos as oscilações de Friedel no ponto fixo de baixa temperatura $T \ll T_{K}$. Identificamos os pontos de interferência construtiva com valores $k_{F} R=(2 n+1) \pi$ e os de interferência destrutiva próximos a valores de $k_{F} R$ múltiplos de $\pi / 4$.

Na sequência, analisamos o comportamento da taxa de relaxação vetorial como função da temperatura nos nós das oscilações que marcam a transição entre as situações de interferência construtiva e destrutiva (figuras 5.5, 5.6 e 5.7, respectivamente). Um aspecto interessante da dependência térmica diz respeito aos picos de de Broglie captados pela taxa de relaxação. Esses picos estão associados à manifestação da interferência quântica entre os estados acoplados à impureza e à ponta de prova.

Observamos uma mudança na dependência térmica de $\left(1 / T_{1}\right)_{\text {vet }}$ quando passamos por $T_{K}$, de modo que usando a relação $R_{K} \propto T_{K}^{-1}$, esse resultado nos mostra que a taxa de relaxação é diferente conforme estamos dentro, fora ou na vizinhança do raio da nuvem Kondo. Em particular, no interior da nuvem e para altas temperaturas, a dependência térmica de $\left(1 / T_{1}\right)_{v e t}$ pode ser mapeada nas curvas universais que descrevem a condutância em pontos quânticos: para a transição de interferência construtiva-destrutiva, o mapeamento é feito com 
a condutância $\mathcal{G}_{\text {side }}$ de um side-coupled quantum dot e, no caso destrutiva-construtiva, a taxa de relaxação é semelhante à condutância $\mathcal{G}_{S E T}$ de um transístor de um único elétron. $\mathrm{Na}$ região supostamente externa à nuvem, passados os picos de de Broglie, a taxa de relaxação tende a um patamar constante - o que fisicamente, indica que, fora da nuvem, há troca de momento magnético da ponta de prova com um único estado metálico, o sítio $f_{1}$. A distâncias próximas a $R_{K}$, o comportamento térmico de $\left(1 / T_{1}\right)_{v e t}$ é caracterizado por uma mistura das curvas observadas a distâncias $R<R_{K}$ e $R>R_{K}$. O efeito da transição do perfil $\mathcal{G}_{\text {side }}$ para $\mathcal{G}_{S E T}$ é mais proeminente quando consideramos o caso de mistura entre os comportamentos destrutivo e construtivo $\left(k_{F} R=(n+1 / 4) \pi\right.$, com $\left.n \in \mathbb{Z}\right)$.

Finalmente, sumarizamos nossa análise da dependência térmica da taxa de relaxação identificando o interior, o exterios e as vizinhanças da da nuvem Kondo (figura 5.8). Verificamos que a mudança é sutil na faixa $<10^{3} k_{F} R<10^{6}$, de modo que nessa região a dependência espacial da taxa de relaxação varia muito pouco e sugere que os efeitos de espalhamento não estão restritos à superfície da nuvem com raio $R_{K}$, mas podem afetar uma faixa de distâncias na vizinhança da nuvem.

Em síntese, conseguimos mostrar que a curva de relaxação do termo vetorial está associada à interferência entre os estados acoplados à ponta de prova e à impureza e, a partir da análise das dependências espacial e térmica desta componente, inferimos que a relação relação $R_{K}=\hbar v_{F} / k_{B} T_{K}$ é verificada para a estimativa do raio da nuvem Kondo. Considerando as oscilações de Friedel da taxa de relaxação nos casos de interferência construtiva e destrutiva, verificamos ainda que há uma região em torno de $R_{K}$ onde os efeitos de espalhamento ainda são importantes.

Esperamos que incluindo a taxa de relaxação total $1 / T_{1}$, incluindo o termo matricial relevante para $R$ pequeno, seja possível incluir os efeitos de superfície aos quais a ponta de ESR seria sensível e, assim, também daria informação sobre a dinâmica da nuvem Kondo. Nesse sentido, trabalhos futuros incluem o cálculo numérico da taxa $\left(1 / T_{1}\right)_{\text {mat }}$ e a análise conjunta dos termos vetorial e matricial em grandes e pequenas distâncias.

Outra investigação interessante diz respeito à geometria. Poderíamos considerar um sistema finito e ver o que acontece com a taxa de relaxação quando a nuvem se forma muito próxima a superfície (situação análoga à ilustrada pela figura 2.3). Uma vez que o procedimento proposto neste trabalho pode ser generalizado, poderíamos investigar também simetrias mais complexas e sistemas com mais impurezas. 


\section{REFERÊNCIAS}

1 KOUWEnhoVen, L. P.; GLAZMAN, L. Revival of the Kondo effect. Physics World, Bristol, v. 14, n. 1, p. 33-38, Jan. 2001.

2 KONDO, J. Resistance minimum in dilute magnetic alloys. Progress of Theoretical Physics, Kyoto, v. 32, n. 1, p. 37-49, 1964. doi: 10.1143/PTP.32.37.

3 GOLDHABER-GORDON, D.; GÖRES, J.; SHTRIKMAN, H.; MAHALU, D.; MEIRAV, U.; KASTNER, M. The Kondo effect in a single-electron transistor. Materials Science and Engineering B, v. 84, n. 1-2, p. 17 - 21, 2001. doi: 10.1016/S0921-5107(01)00550-5.

4 OHIO UNIVERSITY. Double quantum dots control Kondo effect in nanoscience study, 2006. Disponivel em: http://www.sciencedaily.com/releases/2006/09/060914181605.htm. Acesso em: 10 dez. 2013.

5 BOYCE, J. B.; SLICHTER, C. P. Conduction-electron spin density around Fe impurities in $\mathrm{Cu}$ above and below $T_{\mathrm{k}}$. Physical Review Letters, v. 32, n. 2, p. 61-64, Jan. 1974. doi: 10.1103/PhysRevLett.32.61.

6 DE HAAS, W.; DE BOER, J.; DEN BERG, G. The electrical resistance of gold, copper and lead at low temperatures. Physica, v. 1, n. 7-12, p. $1115-1124$, 1934. doi: 10.1016/S00318914(34)80310-2.

7 DE HAAS, W.; VAN DEN BERG, G. Further experiments on the minimum in the resistancetemperature curve of gold. Physica, v. 4, n. 8, p. 683-694, Aug. 1937. doi: 10.1016/S00318914(37)80167-6.

8 VAN DER BERG, G. J. Anomalies in dilute metallic solutions of transition metals. In: DAUNT, J.; EDWARDS, D.; MILFORD, F.; YAQUB, M. (Eds.) Low Temperature Physics LT9. Columbus, Ohio: Springer US, 1965. p. 955-984. doi: 10.1007/978-1-4899-6443-4_64.

9 ANDERSON, P. W. A poor man's derivation of scaling laws for the Kondo problem. Journal of Physics C: solid state physics, v. 3, n. 12, p. 2436, 1970.

10 WILSON, K. G. The renormalization group: critical phenomena and the Kondo problem. Reviews of Modern Physics, New York, v. 47, n. 4, p. 773-840, Oct. 1975. doi: 10.1103/RevModPhys.47.773. 
11 KRISHNA-MURTHY, H. R.; WILKINS, J. W.; WILSON, K. G. Renormalization-group approach to the Anderson model of dilute magnetic alloys. I. static properties for the symmetric case. Physical Review B, v. 21, n. 3, p. 1003-1043, Feb. 1980. doi: 10.1103/PhysRevB.21.1003.

12 ANDERSON, P. W. Localized magnetic states in metals. Physical Review, v. 124, n. 1, p. 41-53, Oct. 1961. doi: 10.1103/PhysRev.124.41.

13 LI, J.; SCHNEIDER, W.-D.; BERNDT, R.; DELLEY, B. Kondo scattering observed at a single magnetic impurity. Physical Review Letters, v. 80, n. 13, p. 2893-2896, Mar. 1998. doi: $10.1103 /$ PhysRevLett.80.2893.

14 MADHAVAN, V.; CHEN, W.; JAMNEALA, T.; CROMMIE, M. F.; WINGREEN, N. S. Tunneling into a single magnetic atom: spectroscopic evidence of the Kondo resonance. Science, v. 280, n. 5363, p. 567, Apr. 1998. doi: 10.1126/science.280.5363.567.

15 KOUWENhOVEN, L.; MARCUS, C. Quantum dots. Physics World, v. 11, n. 6, p. 35-39, 1998.

16 GLAZMAN, L. I.; RAIKKH, M. É. Resonant Kondo transparency of a barrier with quasilocal impurity states. Soviet Journal of Experimental and Theoretical Physics Letters, v. 47, n. 8, p. 452-455, Apr. 1988.

17 NG, T. K.; LEE, P. A. On-site Coulomb repulsion and resonant tunneling. Physical Review Letters, v. 61, n. 15, p. 1768-1771, Oct. 1988. doi: 10.1103/PhysRevLett.61.1768.

18 GOLDHABER-GORDON, D.; SHTRIKMAN, H.; MAHALU, D.; ABUSCH-MAGDER, D.; MEIRAV, U.; KASTNER, M. A. Kondo effect in a single-electron transistor. Nature, v. 391, n. 6663 , p. 156-159, 1998. doi: 10.1038/34373.

19 SERIDONIO, A. C.; YOSHIDA, M.; OLIVEIRA, L. N. Universal zero-bias conductance through a quantum wire side-coupled to a quantum dot. Physical Review B, v. 80, n. 23, p. 235318, Dec. 2009. doi: 10.1103/PhysRevB.80.235318.

20 YOSHIDA, M.; SERIDONIO, A. C.; OLIVEIRA, L. N. Universal zero-bias conductance for the single-electron transistor. Physical Review B, v. 80, n. 23, p. 235317, Dec. 2009. doi: 10.1103/PhysRevB.80.235317.

21 SERIDONIO, A. C.; YOSHIDA, M.; OLIVEIRA, L. N. Thermal dependence of the zerobias conductance through a nanostructure. EPL (Europhysics Letters), v. 86, n. 6, p. 67006, 2009.

22 BORDA, L. Kondo screening cloud in a one-dimensional wire: numerical renormalization group study. Physical Review B, v. 75, n. 4, p. 041307, Jan. 2007. doi: 10.1103/PhysRevB.75.041307. 
23 AFFLECK, I.; SIMON, P. Detecting the Kondo screening cloud around a quantum dot. Physical Review Letters, v. 86, n. 13, p. 2854-2857, Mar. 2001. doi: 10.1103/PhysRevLett.86.2854.

24 SØRENSEN, E. S.; AFFLECK, I. Scaling theory of the Kondo screening cloud. Physical Review B, v. 53, n. 14, p. 9153-9167, Apr. 1996. doi: 10.1103/PhysRevB.53.9153.

25 BARZYKIN, V.; AFFLECK, I. The Kondo screening cloud: what can we learn from perturbation theory? Physical Review Letters, v. 76, n. 26, p. 4959-4962, June 1996. doi: 10.1103/PhysRevLett.76.4959.

26 PEREIRA, R. G.; LAFLORENCIE, N.; AFFLECK, I.; HALPERIN, B. I. Kondo screening cloud and charge staircase in one-dimensional mesoscopic devices. Physical Review B, v. 77, n. 12, p. 125327, Mar. 2008. doi: 10.1103/PhysRevB.77.125327.

27 BOYCE, J. B.; SLICHTER, C. P. Conduction-electron spin density around Fe impurities in $\mathrm{Cu}$ above and below the Kondo temperature. Physical Review B, v. 13, n. 1, p. 379-396, Jan. 1976. doi: 10.1103/PhysRevB.13.379.

28 PINTO, J. W. M.; FROTA, H. O. ESR of a magnetic probe in the neighborhood of an Anderson impurity. International Journal of Modern Physics B, v. 24, n. 31, p. 6185-6202, 2010. doi: 10.1142/S0217979210057638.

29 SCHRIEFFER, J. R.; WOLFF, P. A. Relation between the Anderson and Kondo Hamiltonians. Physical Review, v. 149, n. 2, p. 491-492, Sept. 1966. doi: 10.1103/PhysRev.149.491.

30 BULLA, R.; COSTI, T. A.; PRUSCHKE, T. Numerical renormalization group method for quantum impurity systems. Reviews of Modern Physics, Woodbury, v. 80, n. 2, p. 395-450, Apr. 2008. doi: 10.1103/RevModPhys.80.395.

31 BETHE, H. A. The electromagnetic shift of energy levels. Physical Review, v. 72, n. 4, p. 339-341, Aug. 1947. doi: 10.1103/PhysRev.72.339.

32 TOMONAGA, S. On a relativistically invariant formulation of the quantum theory of wave fields. Progress of Theoretical Physics, Kyoto, v. 1, n. 2, p. 27-42, 1946. doi: 10.1143/PTP.1.27.

33 SCHWINGER, J. On quantum-electrodynamics and the magnetic moment of the electron. Physical Review, v. 73, n. 4, p. 416-417, Feb. 1948. doi: 10.1103/PhysRev.73.416.

34 FEYNMAN, R. Space-time approach to quantum electrodynamics. Physical Review, v. 76, n. 6, p. 769-789, Sept. 1949. doi: 10.1103/PhysRev.76.769.

35 FEYNMAN, R. The theory of positrons. Physical Review, v. 76, n. 6, p. 749-759, Sept. 1949. doi: 10.1103/PhysRev.76.749. 
36 FEYNMAN, R. P. Mathematical formulation of the quantum theory of electromagnetic interaction. Physical Review, v. 80, n. 3, p. 440-457, Nov. 1950. doi: 10.1103/PhysRev.80.440.

37 DYSON, F. The radiation theories of Tomonaga, Schwinger, and Feynman. Physical Review, v. 75, n. 3, p. 486-502, Feb. 1949. doi: 10.1103/PhysRev.75.486.

38 SCHWINGER, J. Selected papers on quantum electrodynamics. Mineola, New York: Dover Publications, 1958.

39 SCHWEBER, S. QED and the men who made it: Dyson, Feynman, Schwinger, and Tomonaga. Princeton, New Jersey: Princeton University Press, 1994.

40 WILSON, K. G. Renormalization group methods. Advances in Mathematics, v. 16, n. 2, p. 170 - 186, 1975. doi: 10.1016/0001-8708(75)90149-8.

41 ANDERSON, P.; YUVAL, G.; HAMANN, D. Scaling theory for the Kondo and onedimensional ising models. Solid State Communications, Oxford, v. 8, n. 13, p. 1033 - 1037, 1970. doi: 10.1016/0038-1098(70)90253-X.

42 YUVAL, G.; ANDERSON, P. W. Exact results for the Kondo problem: one-body theory and extension to finite temperature. Physical Review B, v. 1, p. 1522-1528, Feb. 1970. doi: 10.1103/PhysRevB.1.1522.

43 ABRIKOSOV, A. A.; MIGDAL, A. A. On the theory of the Kondo effect. Journal of Low Temperature Physics, v. 3, n. 5, p. 519-536, 1970. doi: 10.1007/BF00628220.

44 FOWLER, M.; ZAWADOWSKI, A. Scaling and the renormalization group in the Kondo effect. Solid State Communications, Oxford, v. 9, n. 8, p. $471-476,1971$. doi: 10.1016/00381098(71)90324-3.

45 KOMZSIK, L. The lanczos method: evolution and application. Philadelphia, Pennsylvania: Society for Industrial and Applied Mathematics, 2003.

46 CAMPO, V. L.; OLIVEIRA, L. N. Alternative discretization in the numerical renormalization-group method. Physical Review B, v. 72, n. 10, p. 104432, Sept. 2005. doi: $10.1103 /$ PhysRevB.72.104432.

47 CAMPO, V. L.; OLIVEIRA, L. N. Renormalization-group approach to the problem of conduction through a nanostructure. Physical Review B, v. 68, n. 3, p. 035337, July 2003. doi: 10.1103/PhysRevB.68.035337.

48 ABRAMOWITZ, M.; STEGUN, I. Handbook of mathematical functions. 5th. ed. New York: Dover, 1964. 
49 WINTER, J. Magnetic resonance in metals. Oxford: Clarendon Press, 1971.

50 HEWSON, A. The Kondo problem to heavy fermions. Cambridge University Press, 1997.

51 KRISHNA-MURTHY, H. R.; WILSON, K. G.; WILKINS, J. W. Temperature-dependent susceptibility of the symmetric Anderson model: connection to the Kondo model. Physical Review Letters, v. 35, n. 16, p. 1101-1104, Oct. 1975. doi: 10.1103/PhysRevLett.35.1101.

52 KRISHNA-MURTHY, H. R.; WILKINS, J. W.; WILSON, K. G. Renormalization-group approach to the Anderson model of dilute magnetic alloys. I. static properties for the symmetric case. Physical Review B, v. 21, n. 3, p. 1003-1043, Feb. 1980. doi: 10.1103/PhysRevB.21.1003.

53 OLIVEIRA, W. C.; OLIVEIRA, L. N. Generalized numerical renormalization-group method to calculate the thermodynamical properties of impurities in metals. Physical Review B, v. 49, n. 17, p. 11986-11994, May 1994. doi: 10.1103/PhysRevB.49.11986.

54 SAKURAI, J. J. Modern quantum mechanics. 2nd. ed. Reading, Massachussets: AddisonWesley Publishing Company, 1994. 


\section{APÊNDICE $A$}

\section{Relação de completeza}

Vimos na seção 4.2.2 que a taxa de relaxação associada à contribuição matricial pode ser calculada determinando o conjunto de elementos $\left\langle\varphi\left|f_{n}^{\dagger} f_{m}\right| \psi\right\rangle(n, m=0, \ldots, N)$, com $|\varphi\rangle$ e $|\psi\rangle$ sendo estados de um mesmo setor $(q, s)$. Uma forma de identificar as condições para estes estados consiste em inserir a relação de completeza entre os operadores $f_{n}^{\dagger} f_{m}$ e aplicar as regras de seleção (3.38) e (3.39).

A completeza poderia ser útil não apenas para encontrar os $|\varphi\rangle$ e $|\psi\rangle$ contribuindo para a parcela matricial da taxa $\left(1 / T_{1}\right)_{m a t}$, mas também para calcular os elementos de matriz envolvidos. Este procedimento parece vantajoso do vista analítico, dado que a completeza facilita calcular os elementos de matriz, bastando para isso encontrar estados e setores de uma mesma iteração $N$. Infelizmente, essa alternativa falha justamente no regime de interesse, de modo que a taxa de relaxação calculada no regime Kondo apresenta anomalias numéricas e imprecisões.

Este apêndice apresenta os detalhes envolvendo a implementação da completeza e uma breve discussão da razão pela qual precisamos abandoná-la na tentativa de calcular a taxa $\left(1 / T_{1}\right)_{\text {mat }}$.

\section{A.1 Calculando os elementos de matriz de $f_{n \mu}^{\dagger} f_{m \nu}$ por completeza}

Para calcular os elementos de matriz $\left\langle\varphi\left|f_{n \mu}^{\dagger} f_{m \nu}\right| \psi\right\rangle$ pela relação de completeza

$$
\left\langle\varphi\left|f_{n \mu}^{\dagger} f_{m \nu}\right| \psi\right\rangle=\sum_{\xi}\left\langle\varphi\left|f_{n \mu}^{\dagger}\right| \xi\right\rangle\left\langle\xi\left|f_{m \nu}\right| \psi\right\rangle
$$

precisamos encontrar os estados $|\xi\rangle$ para os quais os elementos $\left\langle\varphi\left|f_{n \mu}^{\dagger}\right| \xi\right\rangle$ e $\left\langle\xi\left|f_{m \nu}\right| \psi\right\rangle$ não se anulam.

Na prática, como os estados $|\psi\rangle$, $|\xi\rangle$ e $|\varphi\rangle$ estão conectados por relações entre carga e 
spin, podemos reescrever a expressão (A.1) como sendo

$$
\begin{aligned}
\left\langle q^{\prime}, s^{\prime}, s_{z}^{\prime}, r^{\prime}\left|f_{n \mu}^{\dagger} f_{n^{\prime} \nu}\right| q, s, s_{z}, r\right\rangle= & \sum_{q^{\prime \prime}, s^{\prime \prime}, s_{z}^{\prime \prime}, r^{\prime \prime}}\left\langle q^{\prime}, s^{\prime}, s_{z}^{\prime}, r^{\prime}\left|f_{n \mu}^{\dagger}\right| q^{\prime \prime}, s^{\prime \prime}, s_{z}^{\prime \prime}, r^{\prime \prime}\right\rangle \\
& \times\left\langle q^{\prime \prime}, s^{\prime \prime}, s_{z}^{\prime \prime}, r^{\prime \prime}\left|f_{n^{\prime} \nu}\right| q, s, s_{z}, r\right\rangle \\
= & \sum_{q^{\prime \prime}, s^{\prime \prime}, s_{z}^{\prime \prime}, r^{\prime \prime}}\left\langle q^{\prime}, s^{\prime}, s_{z}^{\prime}, r^{\prime}\left|f_{n \mu}^{\dagger}\right| q^{\prime \prime}, s^{\prime \prime}, s_{z}^{\prime \prime}, r^{\prime \prime}\right\rangle \\
& \times\left\langle q, s, s_{z}, r\left|f_{n^{\prime} \nu}^{\dagger}\right| q^{\prime \prime}, s^{\prime \prime}, s_{z}^{\prime \prime}, r^{\prime \prime}\right\rangle,
\end{aligned}
$$

identificando $\left|q, s, s_{z}, r\right\rangle \operatorname{com}|\varphi\rangle,\left|q^{\prime}, s^{\prime}, s_{z}^{\prime}, r^{\prime}\right\rangle \operatorname{com}|\varphi\rangle$ e $\left|q^{\prime \prime}, s^{\prime \prime}, s_{z}^{\prime \prime}, r^{\prime \prime}\right\rangle$ com $|\xi\rangle$.

Dadas as regras de seleção (3.38) e (3.39), para um estado $\left|q, s, s_{z}, r\right\rangle$ fixo, podemos determinar a carga e o spin dos estados $\left|q^{\prime}, s^{\prime}, s_{z}^{\prime}, r^{\prime}\right\rangle$ e $\left|q^{\prime \prime}, s^{\prime \prime}, s_{z}^{\prime \prime}, r^{\prime \prime}\right\rangle$ pelas seguintes relações:

$$
\begin{aligned}
q & =q^{\prime} \\
s & =s^{\prime} \\
q^{\prime \prime} & =q-1 \\
s^{\prime \prime} & =s \mp \frac{1}{2},
\end{aligned}
$$

e ainda $s_{z}^{\prime}=s_{z}^{\prime \prime}+\mu$ e $s_{z}^{\prime \prime}=s_{z}$.

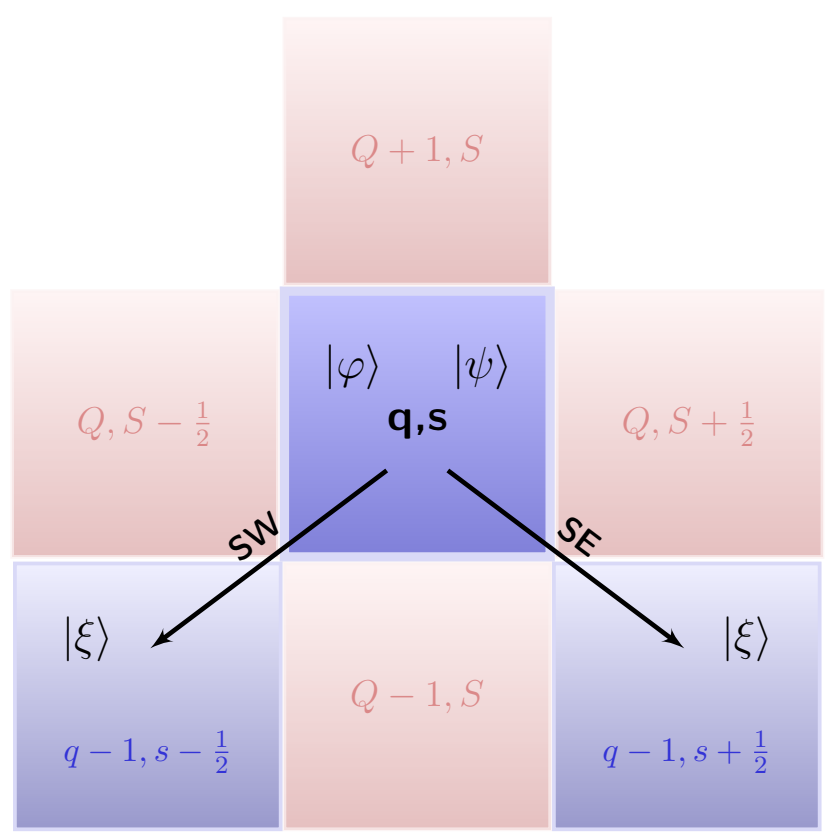

Figura A.1 - Implementação da completeza (A.1) para o cálculo dos elementos de matriz $\left\langle\varphi\left|f_{n \mu}^{\dagger} f_{m \nu}\right| \psi\right\rangle$. Os estados $|\varphi\rangle$ e $|\psi\rangle$ pertencem ao mesmo setor $(q, s)$, enquanto os estados $|\xi\rangle$ podem estar a sudeste $S E$ ou sudoeste $S W$. Fonte: elaborada pela autora. 
A relações (A.3) e (A.4) revelam que os elementos de matriz $\left\langle\varphi\left|f_{n \mu}^{\dagger} f_{m \nu}\right| \psi\right\rangle$ são não-nulos quando os estados $|\varphi\rangle$ e $|\psi\rangle$ pertencem ao mesmo setor $(q, s)$, ao passo que (A.5) e (A.6) determinam que os estados $|\xi\rangle$ da completeza estão localizados a sudeste $S E$ e a sudoeste $S W$ do setor $(q, s)$. Veja a figura A.1.

Uma vez identificados estados que participam do cálculo dos elementos de matriz através da relação de completeza, é uma tarefa simples executar a soma (A.1). Esta última pode ser simplificada pelo Teorema de Wigner-Eckart (3.48), de modo que

$$
\begin{aligned}
\left\langle q^{\prime}, s^{\prime}, s_{z}^{\prime}, r^{\prime}\left|f_{n \mu}^{\dagger} f_{n^{\prime} \nu}\right| q, s, s_{z}, r\right\rangle & =\sum_{r_{S W}^{\prime \prime}}\left\langle r^{\prime}\left\|f_{n}^{\dagger}\right\| r_{S W}^{\prime \prime}\right\rangle\left\langle r\left\|f_{m}^{\dagger}\right\| r_{S W}^{\prime \prime}\right\rangle \\
& \times\left(\begin{array}{r|cc}
s & \frac{1}{2} & s-\frac{1}{2} \\
s_{z}^{\prime} & \mu & s_{z}^{\prime}-\mu
\end{array}\right)\left(\begin{array}{c|cc}
s & \frac{1}{2} & s-\frac{1}{2} \\
s_{z} & \nu & s_{z}-\nu
\end{array}\right) \\
& +\sum_{r_{S E}^{\prime \prime}}\left\langle r^{\prime}|| f_{n}^{\dagger} \| r_{S E}^{\prime \prime}\right\rangle\left\langle r\left\|f_{m}^{\dagger}\right\| r_{S E}^{\prime \prime}\right\rangle \\
& \times\left(\begin{array}{c|cc}
s & \frac{1}{2} & s+\frac{1}{2} \\
s_{z}^{\prime} & \mu & s_{z}^{\prime}-\mu
\end{array}\right)\left(\begin{array}{c|cc}
s & \frac{1}{2} & s+\frac{1}{2} \\
s_{z} & \nu & s_{z}-\nu
\end{array}\right),
\end{aligned}
$$

onde simplificamos a notação dos estados $|r\rangle=|q, s, r\rangle,\left|r^{\prime}\right\rangle=\left|q, s, r^{\prime}\right\rangle,\left|r_{S W}^{\prime \prime}\right\rangle=\left|q-1, s-1 / 2, r^{\prime \prime}\right\rangle$ e $\left|r_{S E}^{\prime \prime}\right\rangle=\left|q-1, s+1 / 2, r^{\prime \prime}\right\rangle$.

Sabemos que o Hamiltoniano da ponta de prova $H_{\text {probe }}$ condiciona a inversão de spin $\nu=-\mu$, de modo que os coeficientes de Clebsch-Gordan da equação (A.1) podem ser expressos na forma

$$
C G_{S W}\left(\mu, s, s_{z}\right)=\left(\begin{array}{c|cc}
s & \frac{1}{2} & s-\frac{1}{2} \\
s_{z}+2 \mu & \mu & s_{z}+\mu
\end{array}\right)\left(\begin{array}{c|cc}
s & \frac{1}{2} & s-\frac{1}{2} \\
s_{z} & -\mu & s_{z}+\mu
\end{array}\right)
$$

e

$$
C G_{S E}\left(\mu, s, s_{z}\right)=\left(\begin{array}{c|cc}
s & \frac{1}{2} & s+\frac{1}{2} \\
s_{z}+2 \mu & \mu & s_{z}-\mu
\end{array}\right)\left(\begin{array}{c|cc}
s & \frac{1}{2} & s+\frac{1}{2} \\
s_{z} & -\mu & s_{z}+\mu
\end{array}\right) .
$$

Considerando os possíveis valores de spin $\mu= \pm \frac{1}{2}$, podemos calcular os valores assumidos pelos coeficientes $C G_{S W}$ e $C G_{S E}$. Os resultados são mostrados nas tabelas A.1 e A.2.

Voltando à taxa de relaxação $\left(1 / T_{1}\right)^{f_{n}}$ em (4.21), devemos ser capazes de calcular o quadrado do elemento de matriz $\left\langle q, s, s_{z}^{\prime}, r^{\prime}\left|f_{n \mu}^{\dagger} f_{m \nu}\right| q, s, s_{z}, r\right\rangle$ levando em conta os possíveis valores de $s_{z}$. 
Tabela A.1 - Coeficientes de Clebsch-Gordan referentes aos elementos de matriz $S W$.

\begin{tabular}{|c|c|c|}
\hline$\mu$ & $\left(\begin{array}{c|cc}s & \frac{1}{2} & s-\frac{1}{2} \\
s_{z}+2 \mu & \mu & s_{z}+\mu\end{array}\right)$ & $\left(\begin{array}{c|cc}s & \frac{1}{2} & s-\frac{1}{2} \\
s_{z} & -\mu & s_{z}+\mu\end{array}\right)$ \\
\hline$+\frac{1}{2}$ & $\sqrt{\frac{s+s_{z}+1}{2 s}}$ & $\sqrt{\frac{s-s_{z}}{2 s}}$ \\
\hline$-\frac{1}{2}$ & $\sqrt{\frac{s-s_{z}+1}{2 s}}$ & $\sqrt{\frac{s+s_{z}}{2 s}}$ \\
\hline
\end{tabular}

Fonte: elaborada pela autora.

Tabela A.2 - Coeficientes de Clebsch-Gordan referentes aos elementos de matriz $S E$.

\begin{tabular}{|c|c|c|}
\hline$\mu$ & $\left(\begin{array}{c|cc}s & \frac{1}{2} & s+\frac{1}{2} \\
s_{z}+2 \mu & \mu & s_{z}+\mu\end{array}\right)$ & $\left(\begin{array}{c|cc}s & \frac{1}{2} & s+\frac{1}{2} \\
s_{z} & -\mu & s_{z}+\mu\end{array}\right)$ \\
\hline$+\frac{1}{2}$ & $-\sqrt{\frac{s-s_{z}}{2 s+2}}$ & $\sqrt{\frac{s+s_{z}+1}{2 s+2}}$ \\
\hline$-\frac{1}{2}$ & $\sqrt{\frac{s+s_{z}}{2 s+2}}$ & $-\sqrt{\frac{s-s_{z}+1}{2 s+2}}$ \\
\hline
\end{tabular}

Fonte: elaborada pela autora.

Reunindo os resultados das tabelas A.1 e A.2 com a equação (A.2), obtemos

$$
\begin{aligned}
\left|\left\langle r^{\prime}\left|f_{n \mu}^{\dagger} f_{m \nu}\right| r\right\rangle\right|^{2}= & \sum_{s_{z}=-s}^{s}\left[\sqrt{\left(s+\frac{1}{2}\right)^{2}-\left(s_{z}+\frac{1}{2}\right)^{2}}+\sqrt{\left(s+\frac{1}{2}\right)^{2}-\left(s_{z}-\frac{1}{2}\right)^{2}}\right]^{2} \\
& \times\left|\left(\frac{1}{2 s}\left\langle r^{\prime}|| f_{n}^{\dagger} f_{m}|| r\right\rangle_{S W}-\frac{1}{2 s+2}\left\langle r^{\prime}|| f_{n}^{\dagger} f_{m} \| r\right\rangle_{S E}\right)\right|^{2},
\end{aligned}
$$


com

$$
\left\langle r^{\prime}\left\|f_{n}^{\dagger} f_{m}\right\| r\right\rangle_{S W}=\sum_{r^{\prime \prime}}\left\langle q, s, r^{\prime}\left\|f_{n}^{\dagger}\right\| q-1, s-\frac{1}{2}, r^{\prime \prime}\right\rangle\left\langle q-1, s-\frac{1}{2}, r^{\prime \prime}\left\|f_{m}\right\| q, s, r\right\rangle
$$

e

$$
\left\langle r^{\prime}\left\|f_{n}^{\dagger} f_{m}\right\| r\right\rangle_{S E}=\sum_{r^{\prime \prime}}\left\langle q, s, r^{\prime}\left\|f_{n}^{\dagger}\right\| q-1, s+\frac{1}{2}, r^{\prime \prime}\right\rangle\left\langle q-1, s+\frac{1}{2}, r^{\prime \prime}\left\|f_{m}\right\| q, s, r\right\rangle
$$

Finalmente, quadrando o termo associados aos Clebsch-Gordan e efetuando a soma sobre $s_{z}$, conseguimos escrever o quadrado do elemento de matriz de interesse como

$$
\left|\left\langle r^{\prime}\left|f_{n \mu}^{\dagger} f_{m \nu}\right| r\right\rangle\right|^{2}=\frac{4}{3} \frac{2 s+1}{2 s(2 s+2)}\left|(2 s+2)\left\langle r^{\prime}|| f_{n}^{\dagger} f_{m}|| r\right\rangle_{S W}-(2 s)\left\langle r^{\prime}|| f_{n}^{\dagger} f_{m} \| r\right\rangle_{S E}\right|^{2} .
$$

Embora o cálculo da taxa $\left(1 / T_{1}\right)_{\text {mat }}$ através da completeza seja relativamente simples de deduzir, sua implementação exige cuidado e, portanto, devemos analisar sua eficácia e precisão. A complexidade do algoritmo que computa o termo à direita da equação (A.13) é $\mathcal{O}\left(N^{2} L^{4}\right)-$ o que torna essa alternativa bastante custosa em tempo de computação e memória exigida para o armazenamento de todos os elementos de matriz envolvidos no cálculo. Outra característica deste método que poderia afetar dramaticamente nossos resultados diz respeito ao erro no cálculo dos invariantes das equações (A.11) e (A.12).

Uma vez que truncamos estados com energia $\lambda_{i}>E_{U V}$, existem imprecisões associadas à computação das taxas de relaxação, pois estas são afetadas pelo conjunto de autovalores e autoestados do Hamiltoniano $H_{N}$. Nas primeiras iterações temos poucos estados e, assim, o truncamento atinge um número pequeno de autovetores. Nas últimas iterações, quando estamos passando para o regime Kondo, o truncamento sucessivo de estados aumenta drasticamente o erro da completeza. Esse efeito torna-se notável devido ao desbalanço energético entre os estados singleto e tripleto, que predominam nos pontos fixos. Uma consequência disso é que o número de estados a sudoeste $(S W)$ e sudeste $(S E)$ não consegue compensar a assimetria. Mesmo impondo que o número de estados no final do truncamento $U V$ seja igual em ambos os setores, as diferenças energéticas envolvendo os autovalores não são suficientes para resolver o problema.

Considerar valores altos para o parâmetro $E_{U V}$ seria uma alternativa computacional para melhorar os resultados da completeza. Embora essa tentativa possa fornecer dados razoáveis, sua consequência imediata seria um enorme aumento no tempo de computação de todo o ciclo iterativo. Assim, devemos buscar outras maneiras para calcular a contribuição matricial. 


\section{Calculando a contribuição matricial por rotação}

Na seção 4.1 mostramos como o cálculo de todo o conjunto de elementos de matriz $\left\langle\varphi\left|f_{n}^{\dagger} f_{m}\right| \psi\right\rangle(n, m=0, \ldots, N)$ pode ser baseado em uma representação matricial cujas linhas representam os operadores $f_{n}^{\dagger}$ e as colunas os operadores $f_{m}$, o que é ilustrado na figura 4.1. Obtivemos a expressão (4.30) relacionando os invariantes $\left\langle\varphi\left\|f_{n}^{\dagger} f_{m}\right\| \psi\right\rangle(n, m=0, \ldots, N)$ aos quatro invariantes $\left\langle\varphi\left\|f_{n \pm 1}^{\dagger} f_{m \pm 1}\right\| \psi\right\rangle$ e definindo uma relação recursiva bi-dimensional. Mostramos que a relação de recorrência pode ser implementada com sucesso se conhecermos os elementos das bordas da matriz 4.1. Assim, calculando os elementos da forma $\left\langle\varphi\left|f_{0}^{\dagger} f_{m}\right| \psi\right\rangle$ $(m=0, \ldots, N-1)$ e $\left\langle\varphi\left|f_{n}^{\dagger} f_{N}\right| \psi\right\rangle(n=0, \ldots, N)$, somos capazes de determinar todos os $\left\langle\varphi\left|f_{n}^{\dagger} f_{m}\right| \psi\right\rangle(n, m=0, \ldots, N)$ que entram no cálculo da taxa de relaxação $\left(1 / T_{1}\right)_{\text {mat }}$ apresentada em (4.21).

Tendo em vista as dificuldades numéricas envolvendo o cálculo dos elementos $\left\langle\varphi\left|f_{n}^{\dagger} f_{m}\right| \psi\right\rangle$ através da relação de completeza, propomos que esta computação seja feita por meio de rotações análogas às da relação (3.83). Nesse procedimento, distinguimos três métodos para calcular os seguintes tipos de elementos: $\left\langle\varphi\left|f_{N}^{\dagger} f_{N}\right| \psi\right\rangle,\left\langle\varphi\left|f_{N}^{\dagger} f_{m}\right| \psi\right\rangle(m=0, \ldots, N)$ e $\left\langle\varphi\left|f_{n}^{\dagger} f_{0}\right| \psi\right\rangle$ $(n=0, . ., N-1)$. Estes são mostrados nas seções B.1, B.1 e B.3.

\section{B.1 Cálculo dos elementos de matriz de $f_{N \uparrow}^{\dagger} f_{N \downarrow}$}

Começamos considerando o cálculo dos invariantes da forma $\left\langle q, s, r^{\prime}\left\|f_{N}^{\dagger} f_{N}\right\| q, s, r\right\rangle$, uma vez que estes são facilmente computáveis a partir dos elementos de matriz de $f_{N \uparrow}^{\dagger} f_{N \downarrow}$ na base primitiva de estados da iteração $N$. Vimos no capítulo 3 que as bases das iterações $N$ e $N-1$ estão relacionadas por

$$
\left|Q, S, S_{z}, p\right\rangle=\mathcal{O}_{g_{p}}\left|q^{\prime}, s^{\prime}, s_{z}^{\prime}, r^{\prime}\right\rangle_{N-1} \quad\left(g_{p}=\mathcal{N}, \mathcal{E}, \mathcal{W}, \mathcal{S}\right)
$$

onde $\left|q^{\prime}, s^{\prime}, r^{\prime}\right\rangle_{N-1}$ denota um autoestado de $H_{N-1}$. 
O elemento de interesse pode então ser reescrito na forma

$$
\begin{aligned}
\left\langle q, s, s_{z}+1, r^{\prime}\left|f_{N \uparrow}^{\dagger} f_{N \downarrow}\right| q, s, s_{z}, r\right\rangle & =\sum_{p p^{\prime}} U\left(p^{\prime}, r^{\prime}\right) U(p, r)\left\langle\mathcal{O}_{g_{p^{\prime}}}\left|f_{N \uparrow}^{\dagger} f_{N \downarrow}\right| \mathcal{O}_{g_{p}}\right\rangle \\
& \times\left\langle\bar{q}, \bar{s}, \bar{s}_{z}+1, \bar{r} \mid \tilde{q}, \tilde{s}, \tilde{s}_{z}, \tilde{r}\right\rangle_{N-1}
\end{aligned}
$$

com $U(p, r)$ sendo o elemento de matriz da transformação unitária que diagonaliza $H_{N}$.

O último fator à direita da equação (B.2) se anula exceto quando $\tilde{q}=\bar{q}, \tilde{r}=\bar{r}$, e $\tilde{s}_{z}=\bar{s}_{z}+1$. A igualdade, então, é reduzida à forma

$$
\left\langle q, s, r^{\prime}\left|f_{N \uparrow}^{\dagger} f_{N \downarrow}\right| q, s, r\right\rangle=\sum_{p p^{\prime}} U\left(p^{\prime}, r^{\prime}\right) U(p, r)\left\langle\mathcal{O}_{g_{p^{\prime}}}\left|f_{N \uparrow}^{\dagger} f_{N \downarrow}\right| \mathcal{O}_{g_{p}}\right\rangle .
$$

O invariante na soma também pode se anular de acordo com o gênero dos operadores $\mathcal{O}_{g_{p^{\prime}}}$ e $\mathcal{O}_{g_{p}}$, sendo não-nulo a menos que $p^{\prime}=p$ (os estados estão associados ao mesmo estado herdado e possuem mesmo gênero). Assim, devemos considerar os seguintes elementos de matriz

$$
\begin{gathered}
\left\langle\mathcal{O}_{\mathcal{N}}\left|f_{N \uparrow}^{\dagger} f_{N \downarrow}\right| \mathcal{O}_{\mathcal{N}}\right\rangle=\left\langle\mathcal{O}_{\mathcal{S}}\left|f_{N \uparrow}^{\dagger} f_{N \downarrow}\right| \mathcal{O}_{\mathcal{S}}\right\rangle=0, \\
\left\langle\mathcal{O}_{\mathcal{E}}\left|f_{N \uparrow}^{\dagger} f_{N \downarrow}\right| \mathcal{O}_{\mathcal{E}}\right\rangle=\left(\begin{array}{c|cc}
s & \frac{1}{2} & s-\frac{1}{2} \\
s_{z}+1 & \frac{1}{2} & s_{z}+\frac{1}{2}
\end{array}\right)\left(\begin{array}{c|cc}
s & \frac{1}{2} & s-\frac{1}{2} \\
s_{z} & -\frac{1}{2} & s_{z}+\frac{1}{2}
\end{array}\right)
\end{gathered}
$$

e

$$
\left\langle\mathcal{O}_{\mathcal{W}}\left|f_{N \uparrow}^{\dagger} f_{N \downarrow}\right| \mathcal{O}_{\mathcal{W}}\right\rangle=\left(\begin{array}{c|cc}
s & \frac{1}{2} & s+\frac{1}{2} \\
s_{z}+1 & \frac{1}{2} & s_{z}+\frac{1}{2}
\end{array}\right)\left(\begin{array}{c|cc}
s & \frac{1}{2} & s+\frac{1}{2} \\
s_{z} & -\frac{1}{2} & s_{z}+\frac{1}{2}
\end{array}\right)
$$

Podemos agora calcular explicitamente os coeficientes de Clebsch-Gordan que aparecem em parênteses nas equações (B.5) e (B.6), obtendo

$$
\left\langle\mathcal{O}_{\mathcal{E}}\left|f_{N \uparrow}^{\dagger} f_{N \downarrow}\right| \mathcal{O}_{\mathcal{E}}\right\rangle=\frac{\sqrt{\left(s+s_{z}+1\right)\left(s-s_{z}\right)}}{2 s}
$$

e

$$
\left\langle\mathcal{O}_{\mathcal{W}}\left|f_{N \uparrow}^{\dagger} f_{N \downarrow}\right| \mathcal{O}_{\mathcal{W}}\right\rangle=-\frac{\sqrt{\left(s+s_{z}\right)\left(s-s_{z}+1\right)}}{2 s+2}
$$

respectivamente.

Tais expressões podem ser simplificadas ainda mais através do teorema de Wigner-Eckart. Observando que $f_{N+}^{\dagger} f_{N-}$ é proporcional a um tensor esférico $T_{q}^{(k)}$ de rank $k=1$ como definido 
em (54), podemos introduzir o conjunto

$$
\begin{array}{r}
T_{1}^{1}=-\frac{1}{\sqrt{2}} f_{N \uparrow}^{\dagger} f_{N \downarrow} \\
T_{1}^{0}=-\frac{1}{2}\left(f_{N \uparrow}^{\dagger} f_{N \uparrow}-f_{N \downarrow}^{\dagger} f_{N \downarrow}\right) \\
T_{1}^{-1}=\frac{1}{\sqrt{2}} f_{N \downarrow}^{\dagger} f_{N \uparrow},
\end{array}
$$

satisfazendo as relações de comutação

$$
\left[J_{z}, T_{q}^{(k)}\right]=q T_{q}^{(k)}
$$

e

$$
\left[J_{ \pm}, T_{q}^{(k)}\right]=\sqrt{k(k+1)-q(q \pm 1)} T_{q \pm 1}^{(k)}
$$

Usando o Teorema de Wigner-Eckart, segue que

$$
\left\langle q, s^{\prime}, s_{z}+1\left|f_{N \uparrow}^{\dagger} f_{N \downarrow}\right| q, s, s_{z}\right\rangle=-\sqrt{2}\left(\begin{array}{c|cc}
s^{\prime} & 1 & s \\
s_{z}+1 & 1 & s_{z}
\end{array}\right)\left\langle q, s^{\prime}|| f_{N}^{\dagger} f_{N}|| q, s\right\rangle,
$$

de modo que substituindo os coeficientes de Clebsch-Gordan em parênteses obtemos

$$
\left\langle q, s, s_{z}+1\left|f_{N \uparrow}^{\dagger} f_{N \downarrow}\right| q, s, s_{z}\right\rangle=\sqrt{\frac{\left(s-s_{z}\right)\left(s+s_{z}+1\right)}{s(s+1)}}\left\langle q, s^{\prime}|| f_{N}^{\dagger} f_{N}|| q, s\right\rangle .
$$

Podemos escrever as equações (B.7) e (B.8) de maneira compacta como

$$
\left\langle\mathcal{O}_{\mathcal{E}}|| f_{N}^{\dagger} f_{N}|| \mathcal{O}_{\mathcal{E}}\right\rangle=\frac{1}{2} \sqrt{\frac{s+1}{s}}
$$

e

$$
\left\langle\mathcal{O}_{\mathcal{W}}|| f_{N}^{\dagger} f_{N}|| \mathcal{O}_{\mathcal{W}}\right\rangle=-\frac{1}{2} \sqrt{\frac{s}{s+1}}
$$

Finalmente, substituindo as equações (B.7) e (B.8) à direita de (B.3) podemos sintetizar o cálculo dos elementos de matriz desejados na soma de estados primitivos a seguir:

$$
\begin{aligned}
\left\langle q, s, r^{\prime}|| f_{N}^{\dagger} f_{N}|| q, s, r\right\rangle & =\frac{1}{2 \sqrt{s(s+1)}}\left((s+1) \sum_{p \in \mathcal{E}} U\left(p, r^{\prime}\right) U(p, r)-s \sum_{p \in \mathcal{W}} U\left(p, r^{\prime}\right) U(p, r)\right) \\
& =\sum_{p} U\left(p, r^{\prime}\right) U(p, r) \mathcal{G}_{g_{p}, g_{p}},
\end{aligned}
$$

com a matriz (diagonal) $\mathcal{G}$ definida na tabela B.1. 
Tabela B.1 - Elementos da matriz de coeficientes $\mathcal{G}$ para as possíveis combinações de gêneros. Embora a matriz seja diagonal, de modo que elementos entre estados com mesmo gênero são pressupostamente diferentes de zero, os elementos que efetivamente são não-nulos têm gêneros $\mathcal{E}$ e $\mathcal{W}$.

\begin{tabular}{r|cccc}
\hline \hline$g_{p^{\prime}} \backslash g_{p}$ & $\mathcal{N}$ & $\mathcal{E}$ & $\mathcal{W}$ & $\mathcal{S}$ \\
$\mathcal{N}$ & 0 & 0 & 0 & 0 \\
$\mathcal{E}$ & 0 & $\sqrt{\frac{s+1}{4 s}}$ & 0 & 0 \\
$\mathcal{W}$ & 0 & 0 & $-\sqrt{\frac{s}{4(s+1)}}$ & 0 \\
$\mathcal{S}$ & 0 & 0 & 0 & 0 \\
\hline
\end{tabular}

Fonte: elaborada pela autora.

Prosseguimos considerando elementos de matriz com estados de spins distintos $s$ e $s^{\prime}=$ $s \pm 1$ - o que é permitido pelas regras de seleção para o operador $f_{N \uparrow}^{\dagger} f_{N \downarrow}$. Embora somente os elementos de matriz diagonais, ou seja, elementos de matriz entre autoestados $\operatorname{com} s=s^{\prime}$ e $r=r^{\prime}$ contribuem diretamente para as taxas de relaxação, os invariantes de $f_{0}^{\dagger} f_{n}(n=$ $0, \ldots, N-1)$ são requeridos no cálculo dos invariantes de $f_{0}^{\dagger} f_{n}(n=0, \ldots, N)$ na iteração $N+1$. Na iteração $N=0$, contudo, precisamos calcular todos os invariantes de $f_{N}^{\dagger} f_{N}$.

Analogamente aos elementos $\left\langle q, s, s_{z}, r^{\prime}\left|f_{N \uparrow}^{\dagger} f_{N \downarrow}\right| q, s, s_{z}, r\right\rangle$, partimos da expressão

$$
\begin{aligned}
\left\langle q, s \pm 1, s_{z}, r^{\prime}\left|f_{N \uparrow}^{\dagger} f_{N \downarrow}\right| q, s, s_{z}, r\right\rangle & =\sum_{p p^{\prime}} U\left(p^{\prime}, r^{\prime}\right) U(p, r)\left\langle\mathcal{O}_{g_{p^{\prime}}}\left|f_{N \uparrow}^{\dagger} f_{N \downarrow}\right| \mathcal{O}_{g_{p}}\right\rangle \\
& \times\left\langle\bar{q}, \bar{s}, d \bar{s}_{z}+1, \bar{r} \mid \tilde{q}, \tilde{s}, \tilde{s}_{z}, \tilde{r}\right\rangle_{N-1},
\end{aligned}
$$

mostrando que os estados primitivos devem ter o mesmo pai $\left|\bar{q}, \bar{s}, \bar{s}_{z}+1, \bar{r}\right\rangle=\left|\tilde{q}, \tilde{s}, \tilde{s}_{z}, \tilde{r}\right\rangle$.

Simplificando a equação (B.17), obtemos

$$
\left\langle q, s \pm 1, s_{z}, r^{\prime}\left|f_{N \uparrow}^{\dagger} f_{N \downarrow}\right| q, s, s_{z}, r\right\rangle=\sum_{p p^{\prime}}^{\prime} U\left(p^{\prime}, r^{\prime}\right) U(p, r)\left\langle\mathcal{O}_{g_{p^{\prime}}}\left|f_{N \uparrow}^{\dagger} f_{N \downarrow}\right| \mathcal{O}_{g_{p}}\right\rangle
$$

onde o primeiro termo é útil para nos lembrar que $p$ e $p^{\prime}$ devem compartilhar o pai, mesmo pertencendo a diferentes setores $(q, s)$ e $(q, s+1)$, como ilustrado na figura B.1.

Agora, mostraremos que equação (B.18) pode ser simplificada mais uma vez. Para tanto, é conveniente distinguirmos os casos $s^{\prime}=s+1$ e $s^{\prime}=s-1$, considerando cada um separadamente. 
B.1.0.1 $s^{\prime}=s+1$

Nossa tarefa é calcular o elemento de matriz $\left\langle q, s+1, s_{z}+1, p^{\prime}\left|f_{N \uparrow}^{\dagger} f_{N \downarrow}\right| q, s, s_{z}, p\right\rangle=\left\langle q, s+\frac{1}{2}, s_{z}+\frac{3}{2} \pm \frac{1}{2}, r\left|\mathcal{O}_{\mathcal{E}} f_{N \uparrow}^{\dagger} f_{N \downarrow} \mathcal{O}_{\mathcal{W}}\right| q, s+\frac{1}{2}, s_{z}+\frac{1}{2} \pm \frac{1}{2}, r\right\rangle$

com $r$ sendo o índice do estado pai.

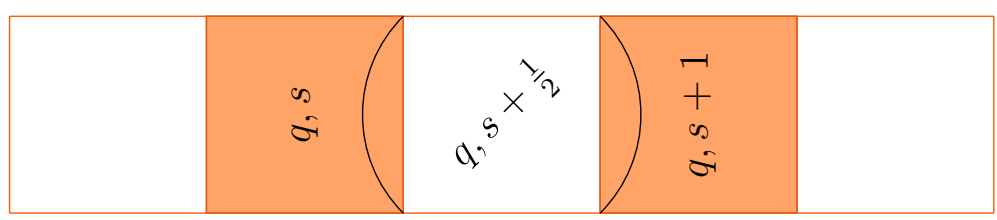

Figura B.1 - Setores contendo estados que contribuem direta ou indiretamente para o invariante $\left\langle q, s+1, r^{\prime}\left\|f_{N}^{\dagger} f_{N}\right\| q, s, r\right\rangle$. Uma vez que os dois estados primitivos (coloridos em laranja) devem ter o mesmo pai, o estado que lhes deu origem deve pertencer ao setor $(q, s+1 / 2)$ (indicado pelo rótulo inclinado $s+1 / 2)$. As abas representam os gêneros dos estados primitivos. Fonte: elaborada pela autora.

A equação (B.19) pode ser reescrita como

$$
\left\langle q, s+1, s_{z}+1, p^{\prime}\left|f_{N \uparrow}^{\dagger} f_{N \downarrow}\right| q, s, s_{z}, p\right\rangle=\left(\begin{array}{c|cc}
s+1 & \frac{1}{2} & s+\frac{1}{2} \\
s_{z}+1 & \frac{1}{2} & s_{z}+\frac{1}{2}
\end{array}\right)\left(\begin{array}{c|cc}
s & \frac{1}{2} & s+\frac{1}{2} \\
s_{z} & -\frac{1}{2} & s_{z}+\frac{1}{2}
\end{array}\right),
$$

ou ainda com a forma explícita dos coeficientes de Clebsch-Gordan, como

$$
\left\langle q, s+1, s_{z}+1, p^{\prime}\left|f_{N \uparrow}^{\dagger} f_{N \downarrow}\right| q, s, s_{z}, p\right\rangle=\frac{\sqrt{\left(s+s_{z}+1\right)\left(s+s_{z}+2\right)}}{2 s+1} .
$$

Do Teorema de Wigner-Eckart, temos a relação

$$
\left\langle q, s+1, s_{z}+1, p^{\prime}\left|f_{N \uparrow}^{\dagger} f_{N \downarrow}\right| q, s, s_{z}, p\right\rangle=-\left\langle q, s+1, p\left\|f_{N}^{\dagger} f_{N}\right\| q, s, p\right\rangle \sqrt{\frac{2\left(s+s_{z}+1\right)\left(s+s_{z}+2\right)}{(2 s+1)(2 s+2)}} .
$$

Comparando com a equação(B.21), somos capazes de obter

$$
\left\langle q, s+1, p^{\prime}\left\|f_{N}^{\dagger} f_{N}\right\| q, s, p\right\rangle=-\sqrt{\frac{2 s+2}{2(2 s+1)}} .
$$


B.1.0.2 $s^{\prime}=s-1$

Para um estado $\left|q^{\prime}, s^{\prime}, s_{z}^{\prime}, p^{\prime}\right\rangle$ tem $s^{\prime}=s-1$, devemos calcular o elemento de matriz $\left\langle q, s-1, s_{z}+1, p^{\prime}\left|f_{N \uparrow}^{\dagger} f_{N \downarrow}\right| q, s, s_{z}, p\right\rangle=\left\langle q, s-\frac{1}{2}, s_{z}+\frac{3}{2} \pm \frac{1}{2}, r\left|\mathcal{O}_{\mathcal{W}} f_{N \uparrow}^{\dagger} f_{N \downarrow} \mathcal{O}_{\mathcal{E}}\right| q, s-\frac{1}{2}, s_{z}+\frac{1}{2} \pm \frac{1}{2}, r\right\rangle$.

Resgatando as definições de $\mathcal{O}_{\mathcal{E}}$ and $\mathcal{O}_{\mathcal{W}}$ para os operadores à direita da equação (B.24), esta fica reescrita como

$$
\left\langle q, s-1, s_{z}+1, p^{\prime}\left|f_{N \uparrow}^{\dagger} f_{N \downarrow}\right| q, s, s_{z}, p\right\rangle=\left(\begin{array}{c|cc}
s-1 & \frac{1}{2} & s-\frac{1}{2} \\
s_{z}+1 & \frac{1}{2} & s_{z}+\frac{1}{2}
\end{array}\right)\left(\begin{array}{c|cc}
s & \frac{1}{2} & s-\frac{1}{2} \\
s_{z} & -\frac{1}{2} & s_{z}+\frac{1}{2}
\end{array}\right),
$$

e fornece

$$
\left\langle q, s-1, s_{z}+1, p^{\prime}\left|f_{N \uparrow}^{\dagger} f_{N \downarrow}\right| q, s, s_{z}, p\right\rangle=-\frac{\sqrt{\left(s-s_{z}-1\right)\left(s-s_{z}\right)}}{2 s} .
$$

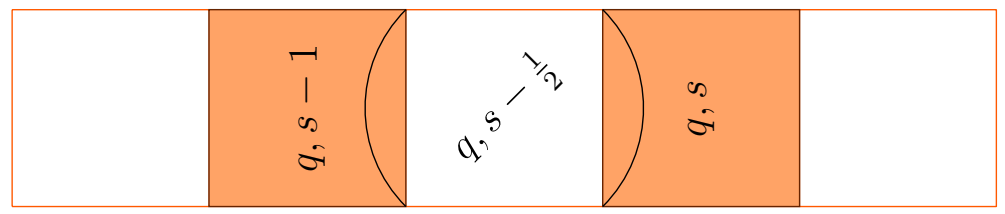

Figura B.2 - Setores envolvidos no cálculo dos elementos de matriz $\left\langle q, s-1, r^{\prime}\left\|f_{N}^{\dagger} f_{N}\right\| q, s, r\right\rangle$. Quando o invariante deve ser tomado entre um ket e um bra de gêneros $\mathcal{E}$ e $\mathcal{W}$, respectivamente, estes foram herdados de estado da iteração $N-1$ que pertencia ao setor $(q, s+1 / 2)$. Fonte: elaborada pela autora.

Utilizando a expressão de Wigner-Eckart

$\left\langle q, s-1, s_{z}+1, p^{\prime}\left|f_{N \uparrow}^{\dagger} f_{N \downarrow}\right| q, s, s_{z}, p\right\rangle=-\left\langle q, s-1, p^{\prime}\left\|f_{N}^{\dagger} f_{N}\right\| q, s, p\right\rangle \sqrt{\frac{2\left(s-s_{z}-1\right)\left(s-s_{z}\right)}{2 s(2 s+1)}}$,

deduzimos os invariantes

$$
\left\langle q, s-1, p^{\prime}\left\|f_{N}^{\dagger} f_{N}\right\| q, s, p\right\rangle=\sqrt{\frac{2 s+1}{4 s}} .
$$




\section{B.2 Cálculo dos elementos de matriz de $f_{m \uparrow}^{\dagger} f_{N \downarrow}$}

Consideremos agora o cálculo dos elementos de matriz ao longo da linha superior da tabela de elementos de matriz que têm a forma $\left\langle q, s, s_{z}+1, r^{\prime}\left|f_{N \uparrow}^{\dagger} f_{m \downarrow}\right| q, s, s_{z}, r\right\rangle(0 \leq m<N)$. Devemos ser capazes de projetar este elemento de matriz na base primitiva da iteração $N$, ou seja,

$$
\left\langle q, s, s_{z}+1, r^{\prime}\left|f_{m \uparrow}^{\dagger} f_{N \downarrow}\right| q, s, s_{z}, r\right\rangle=\sum_{p, p^{\prime}} U\left(p^{\prime}, r^{\prime}\right) U(p, r)\left\langle q, s, s_{z}+1, p^{\prime}\left|f_{m \uparrow}^{\dagger} f_{N \downarrow}\right| q, s, s_{z}, p\right\rangle \text {. }
$$

Dados os gêneros $p$ e $p^{\prime}$, o elemento de matriz que aparece na soma à direita na equação (B.29) é semelhante aos elementos de matriz $\left\langle\tilde{q}, d \tilde{s}, d \bar{s}_{z}+1, p^{\prime}\left|f_{N-1 \uparrow}^{\dagger} f_{N \uparrow}\right| \bar{q}, d \bar{s}, d \bar{s}_{z}, p\right\rangle$ que são computador a partir dos invariantes $\left\langle\bar{q}+1, d \tilde{s}, r\left\|f_{N-1}^{\dagger}\right\| \bar{q}, d \bar{s}, r\right\rangle$ para construir a projeção do Hamiltoniano na base primitiva no início da iteração. Uma vez que os invariantes $\left\langle\bar{q}+1, \tilde{s}, r\left\|f_{m}^{\dagger}\right\| \bar{q}, \bar{s}, r\right\rangle(0 \leq m<N-1)$ podem ser calculados recursivamente, o mesmo procedimento dá acesso a todos os $\left\langle q, s, s_{z}+1, p^{\prime}\left|f_{m \uparrow}^{\dagger} f_{N \downarrow}\right| q, s, s_{z}, p\right\rangle(0 \leq m<N)$. Especificamente, temos a seguinte expressão para os elementos da equação (B.29):

$$
\begin{aligned}
\left\langle q, s, s_{z}+1, p^{\prime}\left|f_{m \uparrow}^{\dagger} f_{N \downarrow}\right| q, s, s_{z}, p\right\rangle= & \sqrt{\frac{\left(s-s_{z}\right)\left(s+s_{z}+1\right)}{s(s+1)}} \frac{1}{2} \alpha_{g_{p^{\prime}} g_{p}}(s) \\
& \times\left\langle Q+1, S^{\prime}, R^{\prime}\left\|f_{m}^{\dagger}\right\| Q, S, R\right\rangle_{N-1},
\end{aligned}
$$

onde os coeficientes $\alpha_{g_{p^{\prime}} g_{p}}$ dependentes do gênero dos elementos estão listados na tabela B.2.

Tabela B.2 - Coeficientes $\alpha_{g p}$ relacionando os elementos de matriz na (B.30). Das possíveis combinações dos gêneros $g=\mathcal{N}, \mathcal{E}, \mathcal{S}, \mathcal{W}$, os elementos não-nulos têm $p^{\prime} \neq p$.

\begin{tabular}{ccccc}
\hline \hline$g_{p^{\prime}} \backslash g_{p}$ & $\mathcal{N}$ & $\mathcal{E}$ & $\mathcal{W}$ & $\mathcal{S}$ \\
\hline $\mathcal{N}$ & 0 & 0 & 0 & 0 \\
$\mathcal{E}$ & $-\sqrt{\frac{2 s+2}{2 s+1}}$ & 0 & 0 & 0 \\
$\mathcal{W}$ & $-\sqrt{\frac{2 s}{2 s+1}}$ & 0 & 0 & 0 \\
$\mathcal{S}$ & 0 & $\sqrt{\frac{s+1}{s}}$ & $-\sqrt{\frac{s}{s+1}}$ & 0 \\
\hline
\end{tabular}

Fonte: elaborada pela autora.

Em analogia com a relação (B.13), podemos usar o Teorema de Wigne-Eckart para sim- 
plificar a expressão para os invariantes da primeira linha da matriz de elementos. Segue que

$$
\left\langle q, s, r^{\prime}\left\|f_{m}^{\dagger} f_{N}\right\| q, s, r\right\rangle=\frac{1}{2} \sum_{p, p^{\prime}} U\left(p^{\prime}, r^{\prime}\right) U(p, r) \alpha_{g_{p^{\prime}} g_{p}}(s)\left\langle Q+1, S^{\prime}, R^{\prime}\left\|f_{m}^{\dagger}\right\| Q, S, R\right\rangle_{N-1} .
$$

O cálculo do termo à direita da igualdade (B.29) para todos os pares $\left(r^{\prime}, r\right)$ tem complexidade $\mathcal{O}\left(N L^{4}\right)$. Felizmente, precisamos calcular somente elementos de matriz à esquerda tais que $r=r^{\prime}$, o que reduz a ordem da operação para $\mathcal{O}\left(N L^{3}\right)$.

\section{B.3 Cálculo dos lementos de matriz de $f_{0 \uparrow}^{\dagger} f_{n \downarrow}$}

Finalmente, vamos dedicar nossa atenção ao cálculo dos elementos de matriz reduzidos $\left\langle q^{\prime}, s^{\prime}, r^{\prime}\left\|f_{0 \uparrow}^{\dagger} f_{n \downarrow}\right\| q, s, r\right\rangle$ ao longo da primeira coluna da tabela 4.1. Estes exigem um pouco mais de cuidado, pois dependem de invariantes da mesma forma calculados nas iterações anteriores. Além disso, as regras de seleção impõe condições não-triviais sobre os spins dos estados $\left|q^{\prime}, s^{\prime}, s_{z}^{\prime}, r^{\prime}\right\rangle$ e $\left|q, s, s_{z}, r\right\rangle$, de modo que devemos calcular os seguintes elementos: $\left\langle q, s, s_{z}+1, r^{\prime}\left|f_{0 \uparrow}^{\dagger} f_{n \downarrow}\right| q, s, s_{z}, r\right\rangle$ e $\left\langle q, s \pm 1, s_{z}+1, r^{\prime}\left|f_{0 \uparrow}^{\dagger} f_{n \downarrow}\right| q, s, s_{z}, r\right\rangle$.

\section{B.3.1 Elementos de $f_{0 \uparrow}^{\dagger} f_{n \downarrow}$ entre estados com $s=s^{\prime}$ e $s_{z}^{\prime} \neq s_{z}$}

Como nos dois casos das seções precedentes para o cálculo dos $f_{N \uparrow}^{\dagger} f_{N \downarrow}$ e $f_{m \uparrow}^{\dagger} f_{N \downarrow}$, começamos projetando os elementos de matriz de interesse na base primitiva de estados na $N$-ésima iteração, de modo que obtemos

$$
\left\langle q, s, s_{z}+1, r^{\prime}\left|f_{0 \uparrow}^{\dagger} f_{n \downarrow}\right| q, s, s_{z}, r\right\rangle=\sum_{p, p^{\prime}} U(r, p) U\left(r^{\prime}, p^{\prime}\right)\left\langle Q, S, S_{z}+1, p^{\prime}\left|\mathcal{O}_{g_{p^{\prime}}} f_{0 \uparrow}^{\dagger} f_{n \downarrow} \mathcal{O}_{g_{p}}\right| Q, S, S_{z}, p\right\rangle .
$$

Os elementos de matriz são relacionados aos invariantes pelo teorema de Wigner-Eckart, que no presente caso fornece a relação:

$$
\left\langle q, s, s_{z}+1, r^{\prime}\left|f_{0 \uparrow}^{\dagger} f_{n \downarrow}\right| q, s, s_{z}, r\right\rangle=\sqrt{\frac{\left(s-s_{z}\right)\left(s+s_{z}+1\right)}{s(s+1)}}\left\langle q, s, r^{\prime}\left\|f_{0}^{\dagger} f_{n}\right\| q, s, r\right\rangle,
$$

A equação (B.32) também pode ser relacionada aos elementos de matriz reduzidos. Note que calculando o último fator na soma da equação (B.32) para cada gênero dos estados 
primitivos e relacionando o elemento resultante ao invariante pertinente, conseguimos escrever

$$
\left\langle q, s, r^{\prime}\left\|f_{0}^{\dagger} f_{n}\right\| q, s, r\right\rangle=\sum_{p, p^{\prime} \in \mathcal{N}, \mathcal{S}} U(r, p) U\left(r^{\prime}, p^{\prime}\right) \mathcal{A}_{g_{p^{\prime}}, g_{p}},\left\langle Q, S, p^{\prime}\left\|f_{0}^{\dagger} f_{n}\right\| Q, S, p\right\rangle
$$

onde a matriz de coeficientes $\mathcal{A}$ é dependente dos gêneros $g_{p^{\prime}}$ e $g_{p}$.

Em particular, os coeficientes $\mathcal{A}_{g_{p^{\prime}}, g_{p}}$ para os pares de gêneros $g_{p^{\prime}}=g_{p}=\mathcal{N}, \mathcal{S}$ são triviais e iguais a um. A tabela B.3 apresenta os coeficientes para todos os pares de gêneros.

Tabela B.3 - Matriz $\mathcal{A}_{g_{p^{\prime}}, g_{p}}$ na soma à direita da equação (B.34). Além dos termos diagonais, destacamos as combinações $\left(g_{p^{\prime}}, g_{p}\right)=(\mathcal{E}, \mathcal{W})$ e $(\mathcal{W}, \mathcal{E})$.

\begin{tabular}{l|cccc}
\hline \hline$g_{p^{\prime}} \backslash g_{p}$ & $\mathcal{N}$ & $\mathcal{E}$ & $\mathcal{W}$ & $\mathcal{S}$ \\
\hline $\mathcal{N}$ & 1 & 0 & 0 & 0 \\
$\mathcal{E}$ & 0 & $\sqrt{\frac{(2 s-1)(2 s+2)}{2 s(2 s+1)}}$ & $\sqrt{\frac{2}{(2 s+1)(2 s+2)}}$ & 0 \\
$\mathcal{W}$ & 0 & $-\sqrt{\frac{2}{2 s(2 s+1)}}$ & $\sqrt{\frac{2 s(2 s+3)}{(2 s+1)(2 s+2)}}$ & 0 \\
$\mathcal{S}$ & 0 & 0 & 0 & 1 \\
\hline
\end{tabular}

Fonte: elaborada pela autora.

Mostramos na sequência um exemplo detalhado de cálculo dos elementos não-triviais de $\mathcal{A}$ para o caso em que os gêneros $g_{p^{\prime}}=g_{p}=\mathcal{E}$.

\section{B.3.1.1 East-East}

Vamos ilustrar o cálculo dos coeficientes não triviais de $\mathcal{A}$ para a situação na qual os estados $|q, s, r\rangle$ e $\left|q, s, r^{\prime}\right\rangle$ são herdados do setor $(Q, S)$ com gêneros iguais $g_{p^{\prime}}=g_{p}=\mathcal{E}$. Na figura B.3 apresentamos os setores envolvidos no cálculo dos elementos de matriz à esquerda e à direita da equação (B.34).

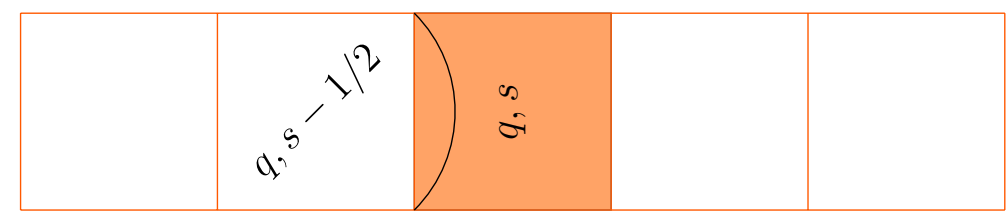

Figura B.3 - Setores envolvidos no cálculo dos elementos de matriz da equação (B.34) para estados com gêneros $g_{p^{\prime}}=g_{p}=\mathcal{E}$. O invariante $\left\langle q, s, p^{\prime}\left\|f_{0}^{\dagger} f_{n}\right\| q, s, p\right\rangle$ é tomado entre estados do mesmo setor $(q, s)$ herdados de $(q, s-1 / 2)$ no início do ciclo iterativo. Fonte: elaborada pela autora. 
Utilizando a identidades (3.59) para o operador $\mathcal{O}_{\mathcal{E}}$, podemos escrever

$$
\begin{aligned}
& \left\langle s-\frac{1}{2}, s_{z}+1 \pm \frac{1}{2}\left|\mathcal{O}_{\mathcal{E}} f_{0 \uparrow}^{\dagger} f_{n \downarrow} \mathcal{O}_{\mathcal{E}}\right| s-\frac{1}{2}, s_{z} \pm \frac{1}{2}\right\rangle=\left\langle s-\frac{1}{2}, s_{z}+\frac{1}{2}\left|f_{N \uparrow} f_{0 \uparrow}^{\dagger} f_{n \downarrow} f_{N \uparrow}^{\dagger}\right| s-\frac{1}{2}, s_{z}-\frac{1}{2}\right\rangle \\
& \times\left(\begin{array}{c|cc}
s & \frac{1}{2} & s-\frac{1}{2} \\
s_{z}+1 & \frac{1}{2} & s_{z}+\frac{1}{2}
\end{array}\right)\left(\begin{array}{c|cc}
s & \frac{1}{2} & s-\frac{1}{2} \\
s_{z} & \frac{1}{2} & s_{z}-\frac{1}{2}
\end{array}\right) \\
& +\left\langle s-\frac{1}{2}, s_{z}+\frac{3}{2}\left|f_{N \downarrow} f_{0 \uparrow}^{\dagger} f_{n \downarrow} f_{N \downarrow}^{\dagger}\right| s-\frac{1}{2}, s_{z}+\frac{1}{2}\right\rangle \\
& \left(\begin{array}{c|cc}
s & \frac{1}{2} & s-\frac{1}{2} \\
s_{z}+1 & -\frac{1}{2} & s_{z}+\frac{3}{2}
\end{array}\right)\left(\begin{array}{c|cc}
s & \frac{1}{2} & s-\frac{1}{2} \\
s_{z} & -\frac{1}{2} & s_{z}+\frac{1}{2}
\end{array}\right) \text {. }
\end{aligned}
$$

Calculando explicitamente os coeficientes de Clebsch-Gordan em parênteses, obtemos

$$
\begin{aligned}
\left\langle s-\frac{1}{2}, s_{z}+1 \pm \frac{1}{2}\left|\mathcal{O}_{\mathcal{E}} f_{0 \uparrow}^{\dagger} f_{n \downarrow} \mathcal{O}_{\mathcal{E}}\right| s-\frac{1}{2}, s_{z} \pm \frac{1}{2}\right\rangle & =\left\langle s-\frac{1}{2}, s_{z}+\frac{1}{2}\left|f_{0 \uparrow}^{\dagger} f_{n \downarrow}\right| s-\frac{1}{2}, s_{z}-\frac{1}{2}\right\rangle \\
& \times \sqrt{\frac{s+s_{z}+1}{2 s}} \sqrt{\frac{s+s_{z}}{2 s}} \\
& +\left\langle s-\frac{1}{2}, s_{z}+\frac{3}{2}\left|f_{0 \uparrow}^{\dagger} f_{n \downarrow}\right| s-\frac{1}{2}, s_{z}+\frac{1}{2}\right\rangle \\
& \times \sqrt{\frac{s-s_{z}-1}{2 s}} \sqrt{\frac{s-s_{z}}{2 s}} .
\end{aligned}
$$

Usando o teorema de Wigner-Eckart, conseguimos relacionar os elementos de matriz da equação (B.36) aos invariantes através da seguinte igualdade:

$$
\begin{aligned}
\left\langle s-\frac{1}{2}, s_{z}+1 \pm \frac{1}{2}\left|\mathcal{O}_{\mathcal{E}} f_{0 \uparrow}^{\dagger} f_{n \downarrow} \mathcal{O}_{\mathcal{E}}\right| s-\frac{1}{2}, s_{z} \pm \frac{1}{2}\right\rangle & =\left\langle s-\frac{1}{2}\left\|f_{0 \uparrow} f_{n \downarrow}\right\| s-\frac{1}{2}\right\rangle \\
& \times\left(\sqrt{\frac{\left(s-s_{z}\right)\left(s+s_{z}\right)}{\left(s-\frac{1}{2}\right)\left(s+\frac{1}{2}\right)}} \sqrt{\frac{s+s_{z}+1}{2 s}} \sqrt{\frac{s+s_{z}}{2 s}}\right. \\
& \left.+\sqrt{\frac{\left(s-s_{z}-1\right)\left(s+s_{z}+1\right)}{\left(s-\frac{1}{2}\right)\left(s+\frac{1}{2}\right)}} \sqrt{\frac{s-s_{z}-1}{2 s}} \sqrt{\frac{s-s_{z}}{2 s}}\right),
\end{aligned}
$$

cuja simplificação resulta em

$$
\begin{aligned}
\left\langle s-\frac{1}{2}, s_{z}+1 \pm \frac{1}{2}\left|\mathcal{O}_{\mathcal{E}} f_{0 \uparrow}^{\dagger} f_{n \downarrow} \mathcal{O}_{\mathcal{E}}\right| s-\frac{1}{2}, s_{z} \pm \frac{1}{2}\right\rangle & =\left\langle s-\frac{1}{2}\left\|f_{0 \uparrow} f_{n \downarrow}\right\| s-\frac{1}{2}\right\rangle \sqrt{\frac{\left(s-s_{z}\right)\left(s+s_{z}+1\right)}{s(s+1)}} \\
& \times \sqrt{\frac{(2 s-1)(2 s+2)}{2 s(2 s+1)}} .
\end{aligned}
$$

Voltando à relação (B.33), somos capazes de identificar a raiz no último termo da equação 
(B.34) como o coeficiente $\mathcal{A}_{g_{\mathcal{E}}, g_{\mathcal{E}}}$. Os demais pares de gêneros que fornecem $\mathcal{A}_{g_{p^{\prime}}, g_{p}}$ não-nulos podem ser calculados por esta mesma sequência de passos.

a)

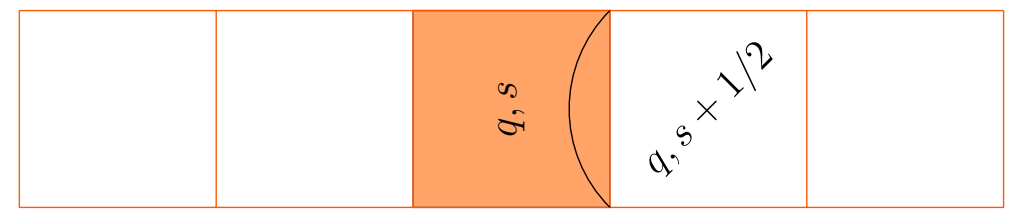

b)

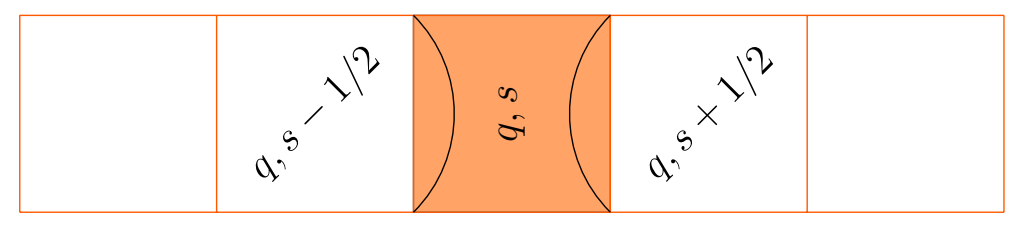

c)

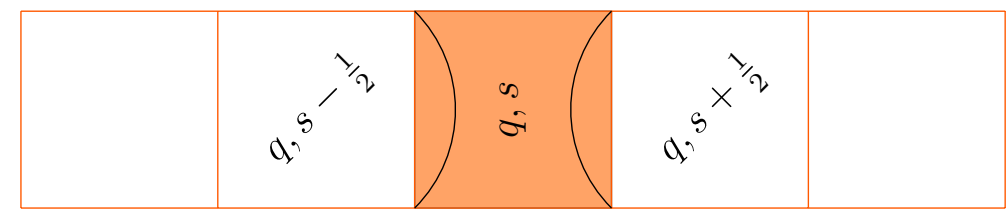

Figura B.4 - Casos especiais de herança para os quais o invariante da da equação (B.34) é não-nulo. São mostrados os seguintes pares de gêneros $g_{p^{\prime}} g_{p}$ : a) $\mathcal{W}-\mathcal{W}$, b) $\mathcal{W}-\mathcal{E}$ e b) $\mathcal{E}-\mathcal{W}$. Fonte: elaborada pela autora.

\section{B.3.2 Elementos de $f_{0 \uparrow}^{\dagger} f_{n \downarrow}$ entre estados com spins diferentes}

Finalmente, precisamos calcular os elementos de matriz de $f_{0 \uparrow}^{\dagger} f_{n \downarrow}$ com spins diferentes. Embora somente os elementos de matriz diagonais $\left\langle q, s, s_{z}+1, r\left|f_{m \uparrow}^{\dagger} f_{n \downarrow}\right| q, s, s_{z}, r\right\rangle(m, n=$ $0, \ldots, N)$ contribuam efetivamente para a taxa de relaxação $\left(1 / T_{1}\right)_{\text {mat }}$, todos os elementos não-nulos de $f_{0 \uparrow}^{\dagger} f_{n \downarrow}$ são necessários, já que a rotação leva da base primitiva para os estados herdados na iteração $N-1$. Dados os setores $\left(q^{\prime}, s^{\prime}\right)$ e $(q, s)$, as regras de seleção permitem elementos de matriz com $q^{\prime}=q$ e $s=s^{\prime}$ (já discutidos na seção B.3.1) e ainda $s= \pm 1$. Estes últimos serão discutidos a seguir.

\section{B.3.2.1 $s^{\prime}=s+1$}

Em analogia com a equação(B.32), temos

$\left\langle q, s+1, s_{z}+1, r^{\prime}\left|f_{0 \uparrow}^{\dagger} f_{n \downarrow}\right| q, s, s_{z}, r\right\rangle=\sum_{p, p^{\prime}}^{\prime} U(r, p) U\left(r^{\prime}, p^{\prime}\right)\left\langle Q, S^{\prime}, S_{z}+1, p^{\prime}\left|f_{0 \uparrow}^{\dagger} f_{n \downarrow}\right| Q, S, S_{z}, p\right\rangle$, 
onde $(Q, S)$ e $\left(Q, S^{\prime}\right)$ indicam, respectivamente, os setores pais dos estados $|q, s, p\rangle$ e $\left|q, s+1, p^{\prime}\right\rangle$.

Novamente, recorremos ao teorema de Wigner-Eckart para escrever os elementos de matriz como função dos invariantes. Segue que

$$
\left\langle q, s+1, s_{z}+1, r^{\prime}\left|f_{0 \uparrow}^{\dagger} f_{n \downarrow}\right| q, s, s_{z}, r\right\rangle=-\sqrt{\frac{2\left(s+s_{z}+1\right)\left(s+s_{z}+2\right)}{(2 s+1)(2 s+2)}}\left\langle q, s+1, r^{\prime}\left\|f_{0}^{\dagger} f_{n}\right\| q, s, r\right\rangle .
$$

Assim como na tabela B.3, os elementos de matriz só não se anulam em duas situações: quando ambos os pais têm o mesmo gênero ou quando um gênero é $\mathcal{E}$ e o outro é $\mathcal{W}$.

Desse modo, os invariantes podem ser obtidos a partir de rotações, valendo a expressão

$$
\left\langle q, s+1, r^{\prime}\left\|f_{0}^{\dagger} f_{n}\right\| q, s, r\right\rangle=\sum_{p, p^{\prime}} U(r, p) \mathcal{B}_{g_{p^{\prime}} g_{p}} U\left(r^{\prime}, p^{\prime}\right)\left\langle Q, S^{\prime}, p^{\prime}\left\|f_{0}^{\dagger} f_{n}\right\| Q, S, p\right\rangle,
$$

com a matrix de coefixientes $\mathcal{B}$ mostrada na tabela B.4.

Tabela B.4 - Matriz de coeficientes $\mathcal{B}_{g_{p^{\prime}}, g_{p}}$ na soma à direita da equação (B.41).

\begin{tabular}{l|cccc}
\hline \hline$g_{p^{\prime}} \backslash g_{p}$ & $\mathcal{N}$ & $\mathcal{E}$ & $\mathcal{W}$ & $\mathcal{S}$ \\
\hline $\mathcal{N}$ & 1 & 0 & 0 & 0 \\
$\mathcal{E}$ & 0 & 1 & $\sqrt{\frac{2}{(2 s+2)(2 s+3)}}$ & 0 \\
$\mathcal{W}$ & 0 & 0 & $\sqrt{\frac{(2 s+1)(2 s+4)}{(2 s+2)(2 s+3)}}$ & 0 \\
$\mathcal{S}$ & 0 & 0 & 0 & 1 \\
\hline
\end{tabular}

Fonte: elaborada pela autora.

\section{B.3.2.2 $s^{\prime}=s-1$}

Em analogia com a equação (B.32), temos a seguinte relação para os elementos de matriz com $s^{\prime}=s-1$ :

$\left\langle q, s-1, s_{z}+1, r^{\prime}\left|f_{0 \uparrow}^{\dagger} f_{n \downarrow}\right| q, s, s_{z}, r\right\rangle=\sum_{p, p^{\prime}}^{\prime} U(r, p) U\left(r^{\prime}, p^{\prime}\right)\left\langle Q, S^{\prime}, S_{z}+1, p^{\prime}\left|f_{0 \uparrow}^{\dagger} f_{n \downarrow}\right| Q, S, S_{z}, p\right\rangle$,

onde, agora, os setores $(Q, S)$ e $\left(Q, S^{\prime}\right)$ são contêm, respectivamente, os estados $|q, s, p\rangle$ and $\left|q, s-1, p^{\prime}\right\rangle$. 
a)

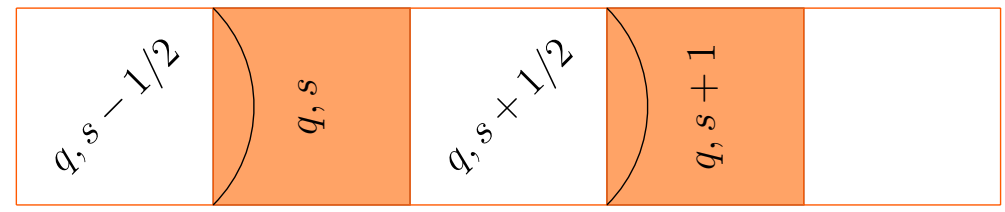

b)

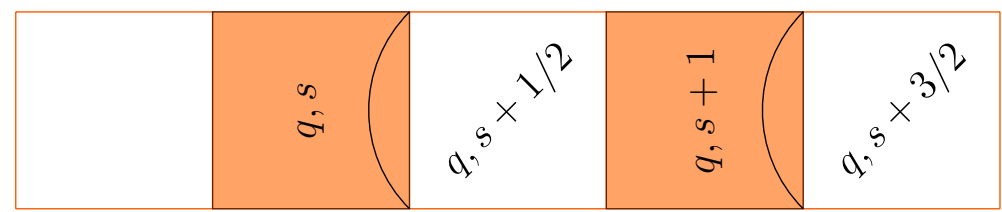

c)

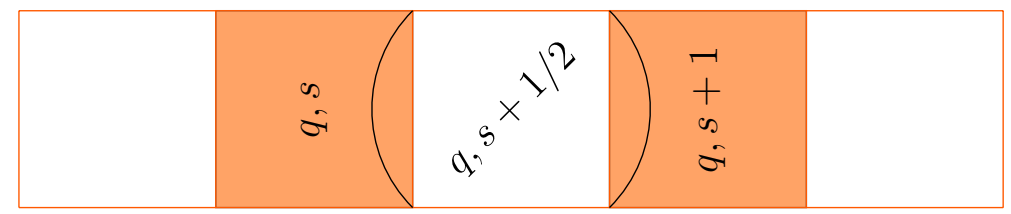

Figura B.5 - Setores $(q, s)$ e $(q, s+1)$ envolvidos no cálculo da equação (B.41). Mostramos os possíveis gêneros dos estados herdados pelos setores pais (com rótulos inclinados): a) $\mathcal{E}-\mathcal{E}$, b) $\mathcal{W}-\mathcal{W}$ e c) $\mathcal{E}-\mathcal{W}$. Fonte: elaborada pela autora.

Com auxílio do teorema de Wigner-Eckart theorem, escrevemos

$$
\left\langle q, s-1, s_{z}+1, r^{\prime}\left|f_{0 \uparrow}^{\dagger} f_{n \downarrow}\right| q, s, s_{z}, r\right\rangle=-\sqrt{\frac{2\left(s-s_{z}-1\right)\left(s-s_{z}\right)}{2 s(2 s+1)}}\left\langle q, s-1, r^{\prime}\left\|f_{0}^{\dagger} f_{n}\right\| q, s, r\right\rangle .
$$

As mesmas condições para que os elementos de matriz não se anulem exploradas nas seções precedentes são válidas. Assim, relacionamos os elementos de matriz desejados aos invariantes da iteração anterior com a seguinte equação:

$$
\left\langle q, s-1, r^{\prime}\left\|f_{0}^{\dagger} f_{n}\right\| q, s, r\right\rangle=\sum_{p, p^{\prime}} U(r, p) \mathcal{C}_{g_{p^{\prime}} g_{p}} U\left(r^{\prime}, p^{\prime}\right)\left\langle Q, S^{\prime}, p^{\prime}\left\|f_{0}^{\dagger} f_{n}\right\| Q, S, p\right\rangle
$$

onde os coeficientes da matriz $\mathcal{C}$ são mostrados na tabela B.5.

Tabela B.5 - Coeficientes $\mathcal{C}_{g^{\prime} g}$ da matriz $\mathcal{C}$ definida à direita da equação (B.44).

\begin{tabular}{l|cccc}
\hline \hline & & & $\mathcal{W}$ & $\mathcal{S}$ \\
$g_{p^{\prime}} \backslash g_{p}$ & $\mathcal{N}$ & 0 & 0 \\
$\mathcal{N}$ & 1 & 0 & 0 & 0 \\
$\mathcal{E}$ & 0 & $\sqrt{\frac{(2 s-2)(2 s+1)}{(2 s-1) 2 s}}$ & & \\
$\mathcal{W}$ & 0 & $\sqrt{\frac{2}{(2 s-1) 2 s}}$ & 1 & 0 \\
$\mathcal{S}$ & 0 & 0 & 0 & 1 \\
\hline
\end{tabular}

Fonte: elaborada pela autora. 
a)

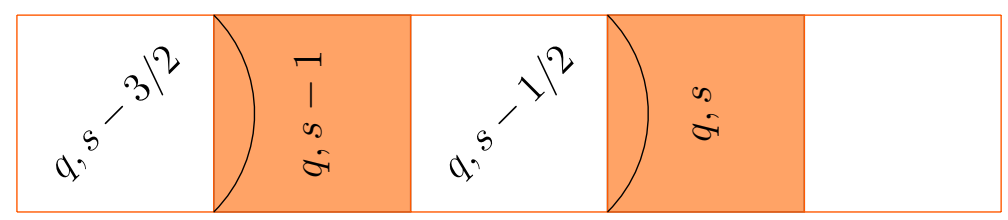

b)

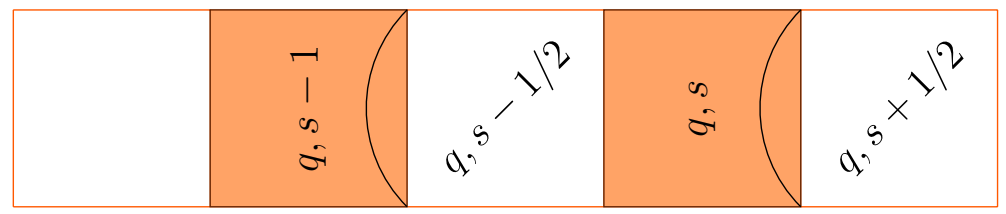

c)

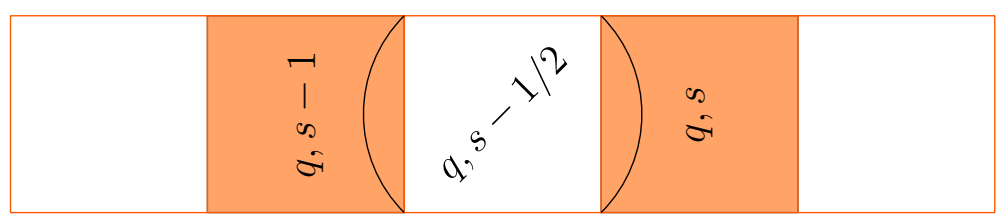

Figura B.6 - Setores $(q, s)$ e $(q, s-1)$ cujos estados participam da relação (B.44). Destacamos os casos não-triviais de herança para os gêneros $g_{p^{\prime}} g_{p}$ : a) $\mathcal{E}-\mathcal{E}$, b) $\mathcal{W}-\mathcal{W}$ e c) $\mathcal{W}-\mathcal{E}$. Fonte: elaborada pela autora. 


\section{Condutâncias em nanoestrututas}

O modelo de Anderson para descrever a interação entre elétrons metálicos e impurezas abriu espaço para o estudo de muitos outros sistemas cujas propriedades físicas pudessem ser extraídas a partir de Hamiltonianos semelhantes. Em particular, avanços em técnicas de microscopia eletrônica favoreceram investigações experimentais e teóricas de propriedades de transporte. Nesse sentido, a concordância entre os resultados é um indicativo de que o modelo de Anderson é bastante poderoso na representação de sistemas físicos em escala nanoscópica, principalmente quando é capaz de reproduzir comportamentos universais.

A condutância em dispositivos nanoscópicos é uma das propriedades de transporte que teve sucesso nos resultados experimentais e teóricos e demonstra como variações do modelo de Anderson são capazes de incorporar a física em nanoestruturas. Além disso, ela é ilustrativa tanto na exploração do conceito de universalidade quanto na validação dos métodos numéricos utilizados na análise teórica.

Este apêndice é dedicado ao estudo da condutância em dois dispositivos quânticos constituídos por fios quânticos acoplados a fios quânticos $(19,20)$. Consideraremos duas geometrias diferentes: um transístor de um único elétron SET e um dispisitivo em formato $T$. Partindo de variantes do modelo de Anderson, mostraremos que a condutância nos dispositivos $T$ e SET pode ser mapeada em funções universais. Para obtê-la numericamente, precisamos obter os autovalores e autovetores do modelo através do Grupo de Renormalização Numérico.

\section{C.1 Condutância universal}

Em ambos os dispositivos, ilustrados na figura C.1, o número de ocupação $n_{d}$ do ponto quântico é controlado pela voltagem $V_{d}$. Quando o número $n_{d}$ é ímpar, o momento magnético do ponto interage antiferromagneticamente com os elétrons de condução do fio, dando origem a um sistema que se comporta analogamente a um sistema Kondo. No regime de baixas temperaturas, o ponto quântico é rodeado pela nuvem Kondo, que pode bloquear ou favorecer a condução eletrônica, denpendendo da geometria do sistema. No arranjo SET, o forte 
acoplamento entre os elétrons de condução e o ponto supera o bloqueo quântico e favorece a condução - que cresce com a diminuição da temperatura. Em especial, nos pontos fixos, o resultado da condutância pelo método NRG é uma função universal $\mathcal{G}_{S E T}\left(T / T_{K}\right)$ da temperatura escalada pela temperatura Kondo $T_{K}$ que descresce monotonicamente. Por outro lado, no dispositivo em formato $T$, a nuvem obstrui o transporte ao longo do fio nas proximidades do ponto quântico. Nos dispositivos menos elaborados, a condutância decai muito rápido para zero quando o sistema é resfriado e encontra-se em temperaturas $T<T_{K}$.
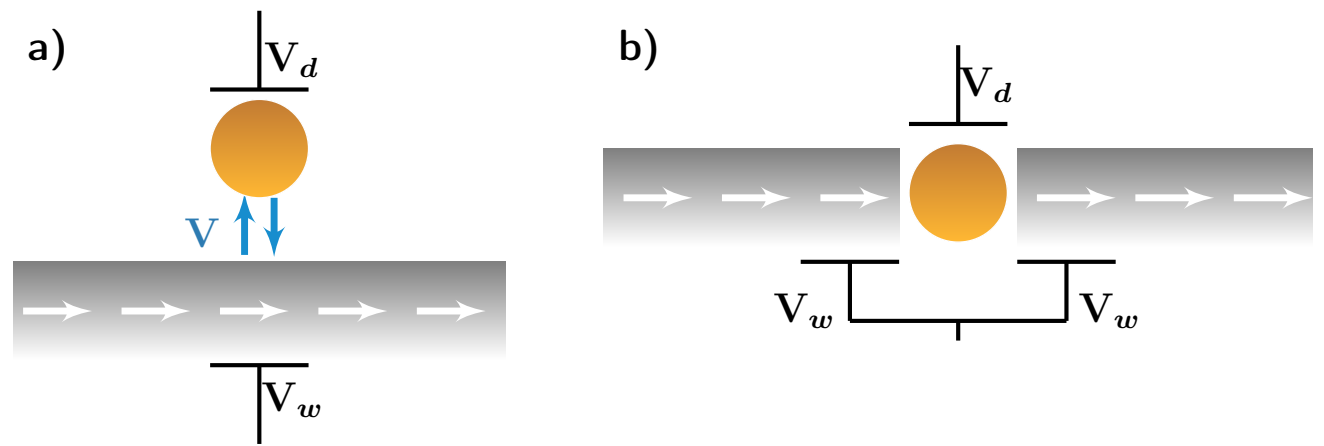

Figura C.1 - Geometrias de ponto quântico acoplado com um fio quântico. a) Dispositivo em formato T. O círculo laranja refere-se a um ponto quântico acoplado lateralmente a um fio quântico. Os potenciais de gate $V_{d}$ e $V_{w}$ controlam, respectivamente, a ocupação eletrônica do ponto e do orbital de Wannier. Esse dispositivo permite que haja interferência entre a corrente fluindo ao longo do fio (setas brancas) e os elétrons que conseguem passar do fio ao ponto. b) Transistor de um único elétron SET. Um ponto quântico faz a ponte entre dois fios quânticos não-interagentes. Um potencial $V_{d}$ controla a energia no ponto quântico, enquanto os potenciais $V_{w}$ deslocam a energia dos orbitais do fio próximo ao ponto. Fonte: elaborado pela autora.

No limite de acoplamento fraco, o sistema pode ser caracterizado essencialmente pelo número de ocupação $n_{d}$. A voltagem $V_{d}$ controla $n_{d}$. Para $V_{d}$ 's adequadamente escolhidos, a ocupação $n_{d}$ pode ser fracionária, o que neutraliza o bloqueio de Coulomb e permite condução.

É portanto interessante analisar como a condutância se comporta como função da voltagem $V_{d}$. Para temperaturas não muito baixas, verifica-se uma sequência de ressonâncias, enquanto para temperaturas muito baixas a condutância apresenta uma sequência de intervalos alternados entre vales e platôs associados, respectivamente, aos comportamentos isolante e condutor. Os platôs são devidos ao efeito Kondo: a medida que o dispositivo é resfriado a temperaturas inferiores a $T_{K} \circ$ momento espalhado produz a ressonância Kondo, capaz de superar o bloqueio Coulombiano e, por conseguinte, permitir condução através do ponto quântico. 
Aplicando um desvio infinitesimal de voltagem ao sistema, podemos obter a seguinte expressão para a condutância

$$
G(T)=\mathcal{G}_{2} \pi \Gamma_{w} \int_{-D}^{D} \rho_{d}(\varepsilon, T)\left[-\frac{\partial f(\varepsilon)}{\partial \varepsilon} d \varepsilon\right]
$$

onde $f(\varepsilon)$ é a função de Fermi, $\mathcal{G}_{2} \equiv 2 e^{2} / h$ é o quantum de condutância, $\Gamma_{W}=\Gamma /(1+$ $\left.\pi^{2} \rho^{2} W^{2}\right)$ é a largura do nível do ponto $c_{d}$ normalizado e $\rho_{d}$ é a densidade espectral do nível energético do ponto quântico.

A seguir, apresentamos os Hamiltonianos das configurações $T$ e SET e mostraremos como a condutância (C.1) é definida em cada uma delas.

\section{C.2 Dispositivo em formato $T$}

O dispositivo lateralmente acoplado em formato $T$ compreende um fio acoplado a um ponto quântico. A corrente no sistema é carregada por elétrons que podem "pular"do fio para o ponto quântico. O parâmetro $V$ mede a amplitude desse processo de transição, tal que para $v \rightarrow \infty$ o sistema emularia um SET. As voltagens $V_{d}$ e $V_{w}$ controlam a ocupação do orbital de Wanier

$$
f_{0} \equiv \frac{1}{\sqrt{N}} \sum_{k} c_{k}
$$

onde $N$ é o número de estados do sistema.

O Hamiltoniano que representa o dispositivo em formato $T$ é dado por

$$
H^{\text {side }}=\overbrace{\sum_{k} \varepsilon_{k} c_{k}^{\dagger} c_{k}+W f_{0}^{\dagger} f_{0}+V\left(f_{0}^{\dagger} c_{d}+H . c .\right)}^{H_{A}}+\overbrace{\left(\varepsilon_{d}+\frac{U}{2}\right) n_{d}-\frac{U}{2} n_{d \uparrow} n_{d \downarrow}}^{H_{d}},
$$

onde $c_{k}\left(c_{k}^{\dagger}\right)$ aniquilam (criam) um elétron com energia $\varepsilon_{k}, V$ é a amplitude de transição entre o fio e o ponto quântico, $U$ representa a repulsão Coulombiana entre os elétrons no orbital do ponto de energia $\varepsilon_{d}$ e $n_{d}$ é o número de ocupação controlado pela voltagem $V_{d}$.

O primeiro termo do Hamiltoniano $H_{A}$ define a banda de condução semi-preenchida com meia-largura $D$. O segundo termo introduz um pontencial de espalhamento controlado pela tensão $V_{w}$. A amplitude de tunelamento $V$ acopla o nível spin-degenerado do ponto quântico ao orbital de Wanier.

Na configuração em formato $T$, a condutância (C.1) no nanodispositivo pode ser escrita 
como

$$
\mathcal{G}_{\text {side }}(T)=\mathcal{G}_{2} \frac{\beta \pi \Gamma}{\mathcal{Z}} \sum_{\varphi, \psi} \frac{\left|\left\langle\varphi\left|f_{0}\right| \psi\right\rangle\right|^{2}}{e^{\beta E_{\varphi}}+e^{\beta E_{\psi}}}
$$

onde $\mathcal{Z}$ é a função de partição, $|\varphi\rangle$ e $|\psi\rangle$ são autoestados de $H^{\text {side }}$ dado em (C.3) com autovalores $E_{\varphi}$ e $E_{\psi}$, respectivamente e $\Gamma \equiv \pi \rho V^{2}$ é a largura do nível eletrônico do ponto quântico devida ao acoplamento com o fio.

\section{C.3 Transístor de um único elétron}

O dispositivo SET pode ser entendido como duas bandas de condução independentes acopladas com um nível localizado. O ponto quântico é assimetricamente acoplado com dois fios - que nada mais são do que gases de elétrons e estão representados por retângulos na figura C.1 b).

Quanticamente, o sistema pode ser representado por uma variante do Hamiltoniano de Anderson. O nível degenerado $c_{d}$ representa o ponto quântico e as duas bandas de condução semi-preenchidas, rotuladas por $L$ (left) e $R$ (right), representam os dois fios quânticos. Cada um dos fios $L(R)$ compreende $N$ estados $c_{k L}\left(c_{k R}\right)$ com energia definida pela relação de dispersão $\varepsilon=\left(k-k_{F}\right) v_{F}$ tal que a largura da banda é $2 D=2 k_{F} v_{F}$, onde $v_{F}$ é a velocidade de fermi. O Hamiltoniano compreende três termos que representam os fios, o ponto quântico e o acoplamento entre os últimos e é escrito como

$$
H^{S E T}=\overbrace{\sum_{k, \alpha} \varepsilon_{k} c_{k \alpha}^{\dagger} c_{k \alpha}+W \sum_{k, q, \alpha} c_{k \alpha}^{\dagger} c_{q \alpha}}^{H_{W}}+\overbrace{V\left(f_{0}^{\dagger} c_{d}+H . c .\right)}^{H_{d}}+\overbrace{\varepsilon_{d} n_{d}+U n_{d \uparrow} n_{d \downarrow}}^{H_{w d}}
$$

onde $W$ é o potencial de espalhamento intra-fio fixado pelo potencial $V_{w}, \alpha=L, R, \varepsilon_{d}$ é a energia do nível do ponto controlada pelo potencial $V_{d}$.

Adaptando a equação (C.1) para a condutância do sistema SET, obtemos a expressão

$$
\mathcal{G}_{S E T}(T)=\mathcal{G}_{2} \frac{\beta \pi \Gamma_{w}}{\mathcal{Z}} \sum_{\varphi, \psi} \frac{\left|\left\langle\varphi\left|c_{d}\right| \psi\right\rangle\right|^{2}}{e^{\beta E_{\varphi}}+e^{\beta E_{\psi}}}
$$

onde $|\varphi\rangle$ e $|\psi\rangle$ são autoestados de $H^{S E T}$ com energias $E_{\varphi}$ e $E_{\psi}$, respectivamente. 


\section{C.4 Resultados numéricos}

Utilizando o método NRG para obter o espectro e o conjunto de autoestados dos Hamiltonianos $H^{\text {side }}$ e $H^{S E T}$ dados, respectivamente, nas equações (C.3) e (C.5), somos capazes de obter numericamente as condutâncias $\mathcal{G}_{\text {side }}$ e $\mathcal{G}_{S E T}$. Na figura C.2 apresentamos os resultados da condutância computada através rotina de diagonalização implementada para este trabalho de mestrado. O cálculo foi executado para três parâmetros de discretização: $\Lambda=3.0, \Lambda=2.5$ e $\Lambda=2.0$.

Os resultados da condutância obtidos numericamente foram utilizados para validar o código que foi desenvolvido para todos as demais etapas deste projeto.

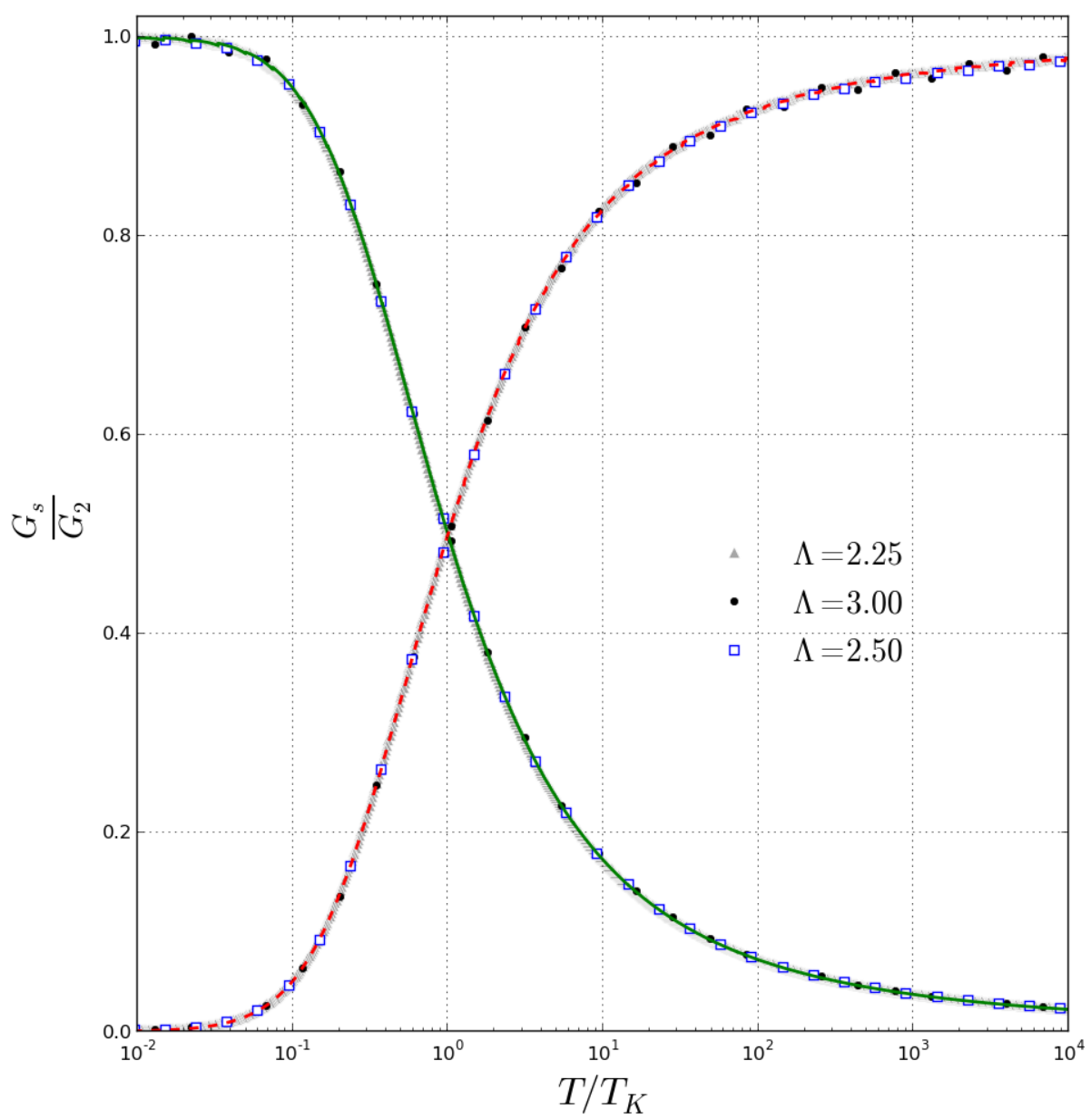

Figura C.2 - Condutâncias $\mathcal{G}_{S E T}$ e $\mathcal{G}_{\text {side }}$ como função de $T / T_{K}$ normalizadas pela condutância quântica $\mathcal{G}_{2}$. A curva vermelha mostra a condutância $\mathcal{G}_{\text {side }}$ através do dispositivo lateralmente acoplado. A medida que a temperatura é resfriada, a condutância decai a zero. Por outro lado, a condutância $\mathcal{G}_{S E T}$, mostrada em verde, cresce para $T<T_{K}$ e tende à unidade no limite $T \rightarrow 0$. Note que para $T=T_{K}$ temos $G\left(T / T_{K}\right)=0.5 \mathcal{G}_{2}$ e as duas condutâncias coincidem. Fonte: elaborado pela autora. 